\title{
A comprehensive review of flow chemistry techniques tailored to the flavours and fragrances industries
}

\author{
Guido Gambacorta, James S. Sharley and lan R. Baxendale*
}

\author{
Review \\ Address: \\ Department of Chemistry, University of Durham, Stockton Road, \\ Durham, DH1 3LE, United Kingdom \\ Email: \\ lan R. Baxendale* - i.r.baxendale@durham.ac.uk \\ * Corresponding author \\ Keywords: \\ flavours and fragrances; flow chemistry; process chemistry; synthesis
}

\author{
Beilstein J. Org. Chem. 2021, 17, 1181-1312. \\ https://doi.org/10.3762/bjoc. 17.90 \\ Received: 29 January 2021 \\ Accepted: 22 April 2021 \\ Published: 18 May 2021 \\ This review is dedicated to Dr. Martin G. Hicks to celebrate his retirement \\ and to thank him for all the work he has done for the Beilstein-Institut and \\ the wider chemical scientific community.
}

Associate Editor: C. Stephenson

(C) 2021 Gambacorta et al.; licensee Beilstein-Institut. License and terms: see end of document.

\begin{abstract}
Due to their intrinsic physical properties, which includes being able to perform as volatile liquids at room and biological temperatures, fragrance ingredients/intermediates make ideal candidates for continuous-flow manufacturing. This review highlights the potential crossover between a multibillion dollar industry and the flourishing sub-field of flow chemistry evolving within the discipline of organic synthesis. This is illustrated through selected examples of industrially important transformations specific to the fragrances and flavours industry and by highlighting the advantages of conducting these transformations by using a flow approach. This review is designed to be a compendium of techniques and apparatus already published in the chemical and engineering literature which would constitute a known solution or inspiration for commonly encountered procedures in the manufacture of fragrance and flavour chemicals.
\end{abstract}

\section{Introduction}

\section{The fragrance industry}

In 2018, the flavours and fragrances $(\mathrm{F} \& \mathrm{~F})$ market was valued $\$ 26.5$ billion with an annual growth of $4.8 \%$, this industry represents a strong economic market expected to be worth more than $\$ 38.5$ billion by 2026 in the rapidly expanding global market [1]. In general, there is an approximate equal split in sales value between the fragrance and flavour sectors with synthetic fragrance ingredients contributing a large proportion of these sales (Figure 1). This, therefore, represents a multibillion dollar outlet for synthetic organic chemistry, creating an arena in which chemists are constantly striving to deliver cheaper and more environmentally friendly processes.

All commercially available products comprised of fragrance ingredients on the market can be traced back to the innovation of fragrance companies as their manufactured products (both of natural and synthetic origin) are sold on to companies further 


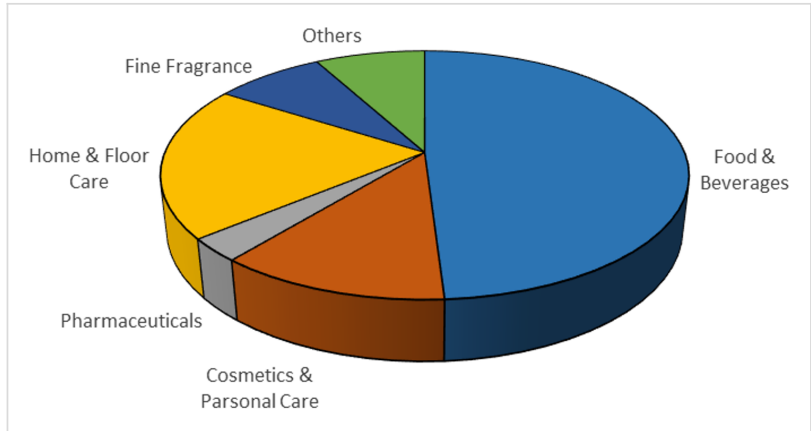

Figure 1: Representative shares of the global F\&F market (2018) segmented on their applications [1].

down the supply chain for formulation, packaging and distribution. International fragrance companies are mainly responsible for the discovery, creation, process development and manufacturing of synthetic fragrance ingredients with all of these representing active areas of current research activity within the field today (Figure 2).

\section{Flow chemistry}

Batch-based synthetic methods have enabled a wide variety of transformations to be performed at industrial scales, however, the implementation of flow-based protocols often lends itself to the creation of superior synthetic systems. Improved processing safety (lowered inventory, improved control of heat/mass transfer), enhanced process diagnostics (PAT, inventory management), upgraded yields and selectivity (improved mixing and energetics) as well as allowing access to expanded processing windows (greater temperature, pressure and reaction time domains) means flow is becoming a popular choice. While recent efforts within the flow chemistry community have been mainly geared towards the use of continuous systems for small/ research scale chemistry, there is growing interest in the translation of such capabilities to larger/commercial scale syntheses [3-7].

\section{Fragrance chemistry}

Since the earliest documented use of fragrances in ancient Egypt and Mesopotamia to current times, the human race has always been fascinated by the manipulation and enhancement of scents. Although nature supplies an abundance of inspiration, the fragrance community has always been apt to try and replicate, or sometimes improve upon the initial resource. One of the first recorded preparations of a synthetic aroma chemical was the reaction of oil of amber with fuming nitric acid, which gave a musky odour as described by scientists at the Berlin Academy in 1759 [8]. By the 20th century, an explosion of new synthetic fragrance discovery had occurred and we now have thousands of different materials, each offering unique and tailored aromas (International FragrancAassociation (IFRA) published a list of active F\&F chemicals) $[9,10]$. Table 1 represents some of the most important synthetic fragrances with their approximate associated prices and tonnages. As can be quickly gleaned a substantial quantity of fragrance materials are manufactured annually. However, some of the listed components are also themselves used to produce other ingredients; for example, myrcene (506) is used for the preparation of linalool (10), geraniol (343), nerol, and 4-(4-Hydroxy-4-methylpentyl)cyclohex-3-ene-1carbaldehyde (lyral, International Flavors \& Fragrance, IFF).

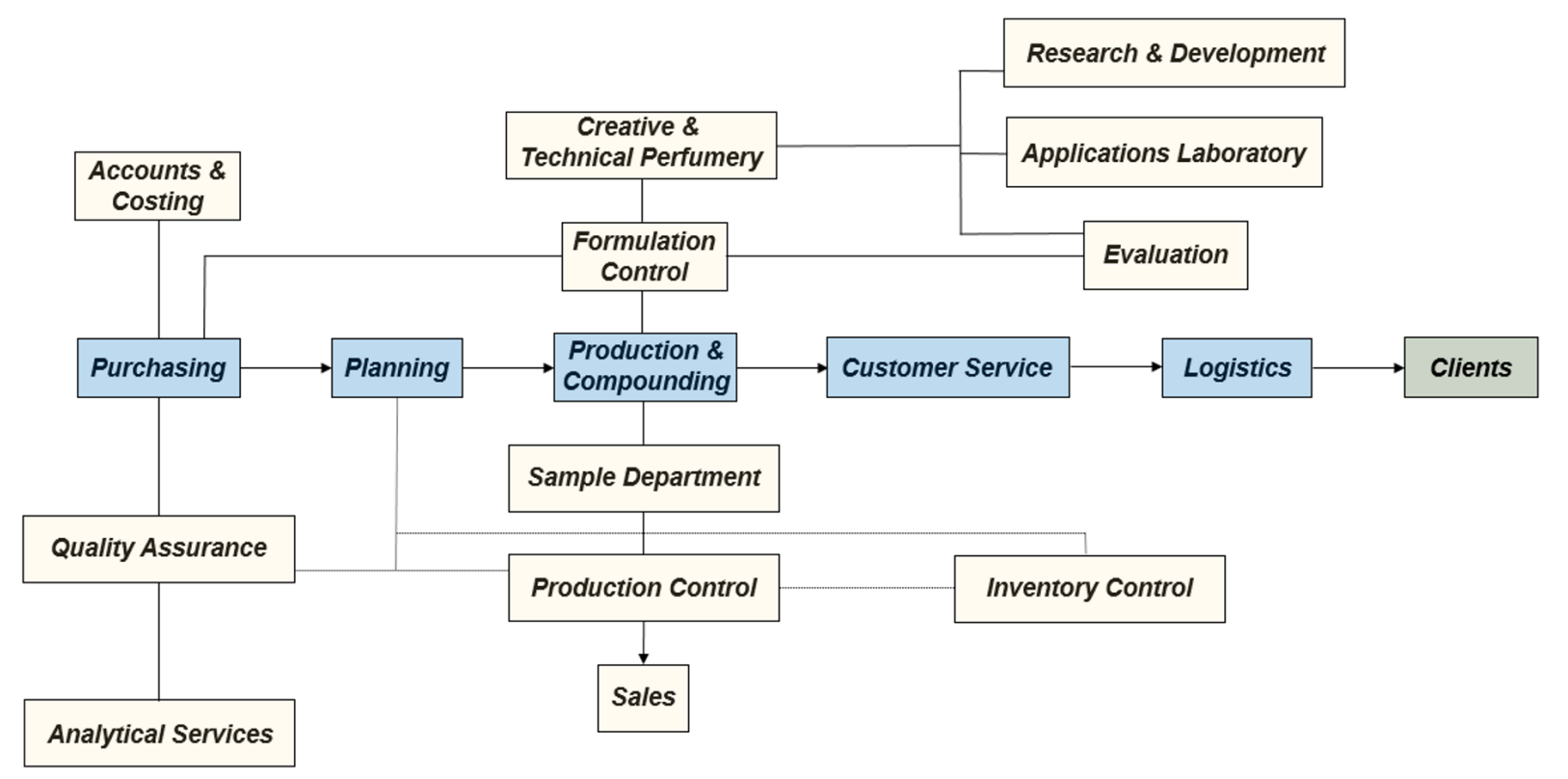

Figure 2: General structure of an international fragrance company [2]. 
Table 1: Some of the most important synthetic fragrance materials $[2,11,12]$.

\begin{tabular}{|c|c|c|c|}
\hline Name & Tonnes per year Annum a & $£ k g^{\mathrm{a}}$ & Odour type \\
\hline myrcene & $30,000^{\mathrm{b}}$ & 1 & balsamic \\
\hline pine oil/terpineol & 30,000 & 1 & pine \\
\hline menthol & 12,000 & 10 & $\operatorname{mint} /$ coolant \\
\hline linalool & 10,000 & 4 & floral/wood \\
\hline citral & 5,000 & 5 & lemonc \\
\hline dihydromyrcene & $5,000^{\mathrm{b}}$ & 1 & - \\
\hline geraniol & 5,000 & 4 & rose \\
\hline (methyl)ionones & 5,000 & 15 & violet \\
\hline dihydromyrcenol & 4,000 & 4 & citrus/floral \\
\hline limonene & 3,500 & 1 & orange $^{\mathrm{d}, \mathrm{e}}$ \\
\hline citronellol (299) & 3,000 & 7 & rose \\
\hline isobornyl acetate $(S, S, S-664)$ & 2,000 & 1 & pine \\
\hline linalyl acetate & 2,000 & 4 & fruity/floral \\
\hline tetrahydrolinalool & 2,000 & 4 & floral \\
\hline carvone & 1,500 & 10 & spearmint \\
\hline hydroxycitronellal & 1,000 & 8 & muguet \\
\hline lyral (IFF) & 1,000 & $12-23^{f}$ & floral \\
\hline terpenylcyclohexanols & 1,000 & $4-38^{f}$ & sandalwood \\
\hline zenolide and analogues & $1,300-4,300$ & $60-250^{f}$ & musk \\
\hline civetone and analogues & $50-500$ & $8-80^{f}$ & musk \\
\hline cedrene derivatives & 500 & 20 & cedarwood \\
\hline Amberlyn ${ }^{\circledR} /$ Ambrox ${ }^{\circledR} /$ Ambroxan ${ }^{\circledR}$ & 50 & $500-750$ & ambergris \\
\hline bangalol and analogues & 30 & 30 & sandalwood \\
\hline
\end{tabular}

aThese prices and volumes are estimated. ${ }^{\mathrm{b}} \mathrm{A}$ substantial proportion of the total consumption is used for manufactured of other ingredients. ${ }^{\mathrm{c}} \mathrm{Citral}$ has little use in fragrance. The tonnage is used for manufacturing ionones and methylionones. ${ }^{\mathrm{d}}$ The material used is actually orange terpenes, which is about $80 \%$ limonene but the odour comes from minor components. ${ }^{\mathrm{e}}$ This figure relates to the use of orange oil in perfumery. About 1,500 tonnes per

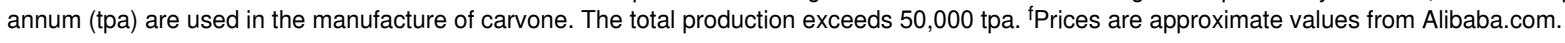

The classification of fragrances to instil order and allow comparison of materials has always been a challenge based upon the vagaries and emotive disparity of the human sense of smell. Several approaches have been proposed and over time superseded by improved scientific models. The Michael Edwards Fragrance wheel (Figure 3) was first introduced in 1983 as a perfumery classification system and is divided into the four standard families: floral, oriental, fresh and woody. Until 2010 fougère was also included as a family constituent, however, it has subsequently been integrated as one of the fourteen subclasses (aromatic). While this can be viewed as an overall scent map based upon the experience of a single perfumer, it has been shown to be highly consistent with other studies and approaches from across the scientific literature [13].

Each principle note can be further subdivided yielding a related family of scents. For example, the oriental family represent odours that would be associated with amber, incense, vanilla and those possessing spicy and resinous characteristics (i.e., 1-3). Woody notes are often associated with scents such as

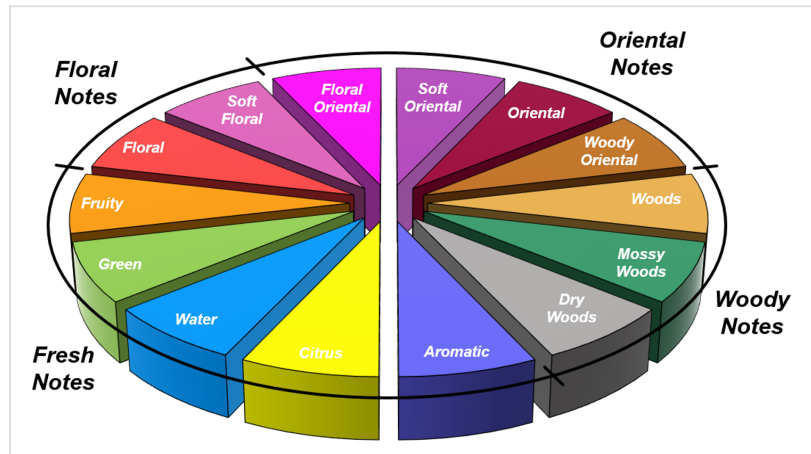

Figure 3: The Michael Edwards fragrance wheel.

cedar, patchouli, pine, sandalwood and vetiver, it also includes the more mossy fragrances, for example, oakmoss (i.e., 4-7). Fresh notes are often associated with ozonic or fruity aromas, orange and lemon zest, bergamot and freshly cut grass (i.e., 8-10). The floral note family represents the most popular family with a vast repertoire of aromas mostly derived from plantbased scents (i.e., 11 and 12). As can be seen the structural 
diversity, including the range of simplicity and complexity represented by the different scent molecules is impressive (Figure 4).

\section{The advantages of a flow approach}

Flow chemistry as a branch of organic synthesis has been reviewed extensively through several informative and educational articles [14-21] and books [22-27]. As such our aim is not to include a comprehensive review here but to simply highlight the principle characteristics and direct the reader to the listed references for further consultation.

In overview a basic depiction of a sequential batch vs flow approach for a multistep synthesis is shown in Figure 5. In the batch process, multiple discreet steps and reaction vessels are required each often associated with an accompanying work-up stage (and purification).
Industrial syntheses often face lengthy, economically arduous synthetic operations that require an extended labour investment to maintain and operate (requirements for large volumes of solvent during work-ups and cleaning of reactors between processes). Alternatively, in the continuous-flow approach, a fully automated reaction system can be assembled, in which, transformations occur within reactors with a continuous interconnected output. The potential inclusion of downstream inlets allows for further reagent addition and direct telescoping into a subsequent reactor/reaction step. This can in theory be repeated as many times as is desired.

This approach is being increasingly adopted to address synthesis problems across both research and manufacturing setting [28-32]. The massive increase in interest towards flow chemistry throughout the field of organic synthesis has been aided in part by the advent and increasing availability of lab-scale flow

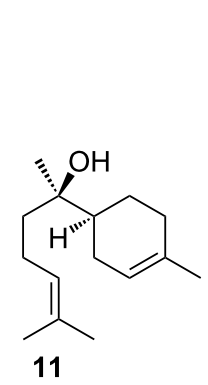

+ -) $\alpha$-bisadolol mild floral, peppery, balsamic, clean

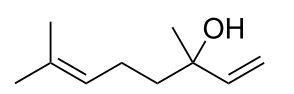

10

linalool waxy, citrus, orange, floral, rose

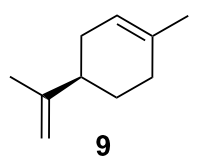

$(+)-(R)$-limonene citrus, orange, fresh sweet<smiles>C=C(C)[C@@H]1CCC2=CC(=O)C[C@H](C)[C@]2(C)C1</smiles>

8

(+)-nootketone grapefruit, peel, citrus, gardenia, woody

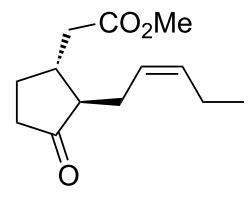

12

(-)-methyl jasmonate jasmin flower, oily, green, petal, soft greasy

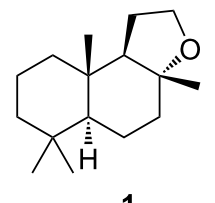

1

(-)-ambrox ambregris, old paper, sweet, labdanum, dry<smiles>COc1cc(C=O)ccc1O</smiles>

vanillin sweet, vanilla, creamy, chocolate
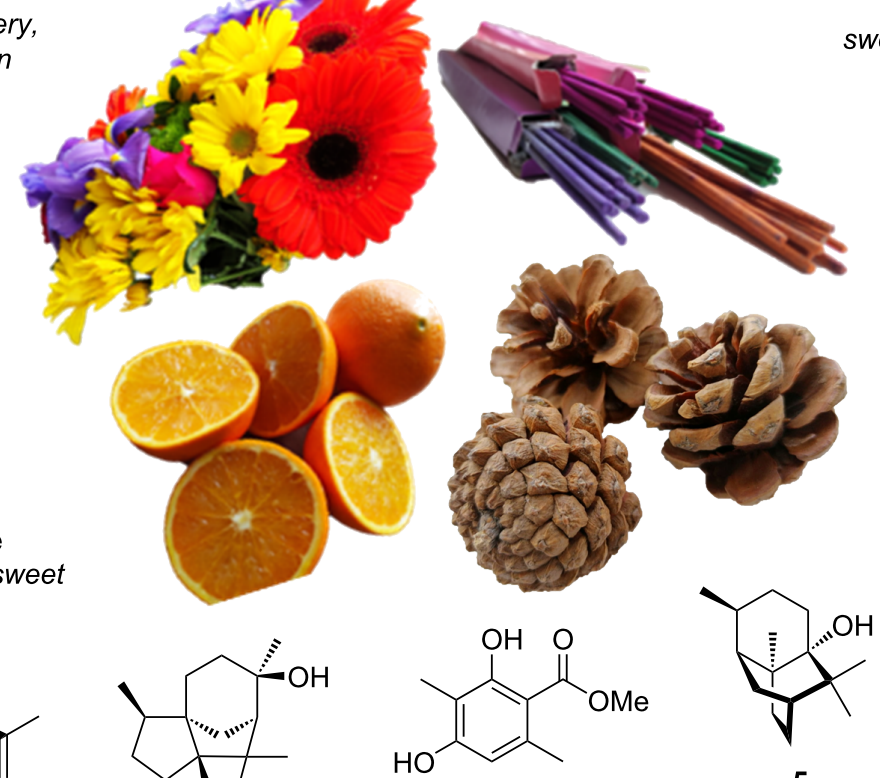

5<smiles>CC1=CC=C[C@]2(C)Cc3occ(C)c3CC12</smiles>

3 furanoeudesma-1,3-diene sweet, warm, leathery-balsamic<smiles>C/C(=C/CCC1(C)C2C=C[C@H](C2)C1(C)C)CO</smiles>

4

(-)-(Z)-ß-santolol woody, cedarwood

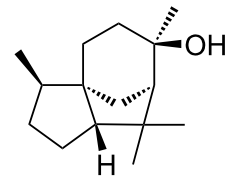

7

(+)-cedrol cedarwood woody, dry, sweet, soft<smiles>COC(=O)c1c(C)cc(O)c(C)c1O</smiles>

6 (-)-patchoulol patchouli, earthy, mossy, oakmoss, woody, camphor powdery phenolic, earthy 


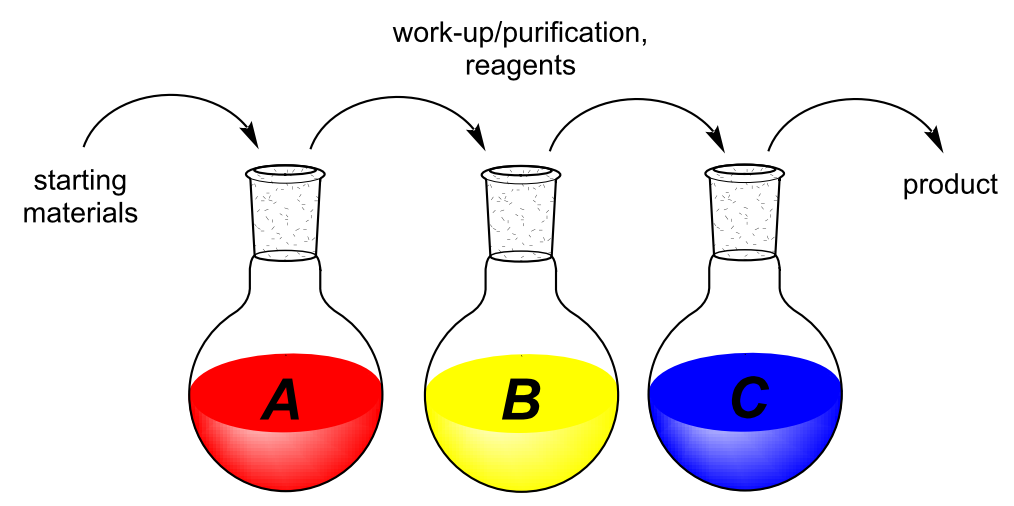

discreet batch processes

vs

continous flow process

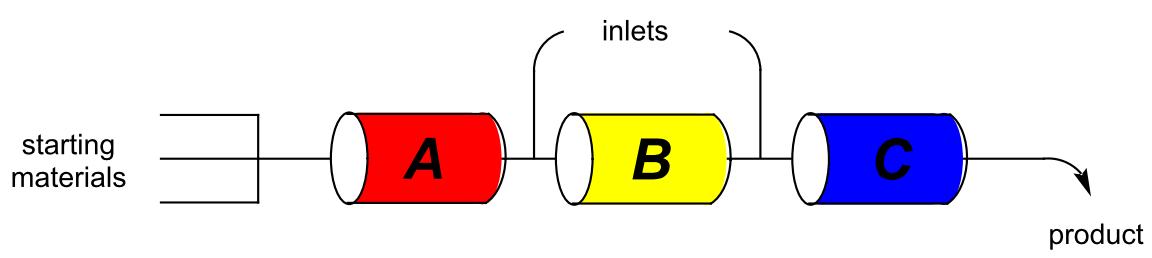

Figure 5: A basic depiction of batch vs flow.

systems designed by both academic and industrial sectors. The commercial flow reactors allow for the safe simulation of larger scale processes within the lab environment, facilitating and expediting the process optimisation experience. While some of the implications of the flow chemistry are more obvious than others, i.e., greener processes with potential cost reduction; there are many potential applications of this field in the small scale chemistry as well. For example, areas like combinatorial chemistry [33-35], complex multistep syntheses [14,36,37], synthesis of pharmaceutical substances have witnessed tremendous growth using flow chemistry approach [14,28,29,38,39]. There are many reasons for adopting flow chemistry and continuous manufacturing practices in both industry and academia: Increased levels of automation, inherent health and safety implications due to containment, the implicit higher heat and mass transfer rates, higher temperature and pressure range access, the potential for in-line purification, monitoring and telescoping, linear scalability and more efficient mixing. These applications are very attractive for a synthetic chemist in all settings, especially considering the current ever-increasing industrial pressure to deliver 'greener' chemical processes at the lowest possible cost.

The 12 principles of green chemistry were introduced in 1998 by Paul Anastas and John Warner [40] and outline what is meant by and to be expected of a green chemical, process or product. Principles for which flow chemistry may be pertinent are displayed by an upward arrow in Table 2, for others only minor implications can be imagined [41].

Later, in 2003 Anastas went on to publish the 12 principles of green engineering [42], the relevance of continuous manufacturing to these is displayed in Table 3. Together these clearly demonstrate the compatibility of the flow approach with a green chemical manufacturing future. It is also not difficult to see how continuous manufacturing has implications for important green chemistry metrics such as atom economy, reaction mass efficiency, effective mass yield, carbon efficiency and environmental (E) factor (sometimes referred to as the Sheldon E Factor) [43]. These parameters are an integral part of contemporary process evaluation frameworks such as the SELECT (safety, economics, legal, environmental, control, throughput) criteria and there is an increasing demand to keep these numbers as close to optimal as possible.

Certain drawbacks to using a flow-approach must also however be acknowledged. Problems may arise due to quenching requirements, the necessity for solvent switching, concentration limitations, compensating for different encountered reaction kinetics over multiple steps when attempting telescoped reaction sequences, potential requirements for intermediate purification and issues arising due to heterogeneity. Hence, a batch or 
Table 2: The 12 principles of green chemistry.

Principle

Flow adherence

prevent waste
atom economy
less hazardous chemical syntheses
design benign chemicals
benign solvents and auxiliaries
design for energy efficiency
use of renewable feed stocks
reduce derivatives
catalysis (vs stoichiometric)
design for degradation
real-time analysis for pollution prevention
inherently benign chemistry for accident prevention

$\uparrow$

$\uparrow$

$\downarrow$

$\uparrow$

$\uparrow$

$\leftrightarrow$

$\leftrightarrow$

$\uparrow$

$\leftrightarrow$

$\uparrow$

$\uparrow$

Table 3: The 12 principles of green engineering.

Principle

Flow adherence

inherent rather than circumstantial

$\uparrow$

prevention instead of treatment

design for separation

maximize efficiency

output-pulled versus input-pushed

conserve complexity

durability rather than immortality

meet need, minimize excess

minimize material diversity

integrate material and energy flows

design for commercial "afterlife"

renewable rather than depleting

semi-batch (cascade CSTR's) approach often offers a more convenient and sometimes superior synthetic approach. Efforts directed towards addressing these drawbacks currently account for a large proportion of the research being conducted within the flow chemistry community. The continuous processing of slurries, for example, is now routinely performed [44-48], also, the use of scavengers (e.g., solid support reagents) [49-51] and devices such as membrane-based continuous separators [52-55] has come a long way in aiding with in-line purification and work-up issues. Table 4 provides a general summary list of advantages and disadvantages of a flow approach.

There are multiple possible configuration and module combinations of mixing elements, reactor residence coils, heating/cooling segments and additional downstream components such as phase separators, quenching stages and crystallisers. A typical flow reactor will comprise two or more pumps (isocratic/peri- staltic/syringe) that feed an HPLC manifold (into which samples may be injected). Separate reagent streams then meet in a variety of ways, the simplest being within a T- or Y-piece. This method of sample stream merging has been demonstrated to give rise to far more efficient mixing than in the case of standard batch reactors, particularly with microreactors (lateral dimensions $<1 \mathrm{~mm}$ ). In batch, inhomogeneities due to poor mixing can lead to convective dead zones, giving rise to concentration gradients and hot spots. Flow microreactors allow for rapid mixing on timescales in the order of $100 \mu$ s and much more efficient heat transfer is made possible by their high surface-to-volume ratios, typically $30,000 \mathrm{~m}^{2} \mathrm{~m}^{-3}$, compared to 100 and $4 \mathrm{~m}^{2} \mathrm{~m}^{-3}$ for laboratory beakers and batch reactors, respectively [56]. Turbulent (Reynold's number $(\mathrm{Re})>4000$, index defining the flow pattern of a fluid) flow profiles are highly desirable, however, mixing issues associated with laminar $(\operatorname{Re}<2000)$ flow profiles may be easily ameliorated by 
Table 4: Flow approach advantage and disadvantages. Advantages

- increased levels of automation

- high heat (surface-to-volume ratio) and mass transfer rates

- potential for in-line purification and telescoping

- compatibility with "forbidden chemistries"

- efficient mixing

- linear scalability and high throughput

- health and safety implications

- facile access to high temperatures and pressures

\section{Disadvantages}

- quenching/work-up issues

- dilution effects of additional downstream flow streams

- solvent limitations for multistep procedures

- inability to compensate for reaction kinetics

- start-up and shut-down procedures

- issues with heterogeneity

- higher training and implementation requirements the incorporation of devices such as mixing chips and structured static mixers [57]. The resultant mixed stream is then directed into a reactor, of which there are many types, these allow for anything from increased simple residence time to active stimulation by application of variable temperature zones, light or microwave irradiation, electrolysis and even high frequency sonication.

The ability to quickly and easily assemble and modify a flow setup allows not only the chemical reaction to be performed as a continuous dynamic flow process but also for direct diagnostics to be obtained facilitating rapid analysis of the content and assessment of the extent of reaction in real-time. Several in-line monitoring tools, for example, ReactIR [58-62] and flow based NMR [63-66] which allow for substrate specific, nonconsuming analysis, have also been developed. Other in-line techniques employing simple UV [67-70], MS [71-73], and Raman [74-77] analysis have also been successfully applied enabling efficient optimisation and generation of new reaction chemistry.

Flow-based chemical processing has elevated other areas of chemistry improving performance and utility, for example, reinvigorating the area of solid-supported reagents [78]. The incorporation of solid reagents is facilitated in flow through the use of packed bed columns and channels. Consequently, the filtration necessary as part of a batch process becomes inherently gratuitous, recycling and regeneration also become easier in flow as reactivation flow streams can be directed through the reactors. There are several other adventitious benefits of using solid-supported reagents in flow, for example, a "local excess" at the entry point of a column reactor is, in certain instances, indicated to give rise to superior reaction kinetics [79]. It is also easy to create staged temperature gradients to compensate for changes in reaction kinetics based upon concentration or complicating effects such as product derived auto-catalysis. The use of immobilised reagents has also greatly aided in the development of impressive sequential multistep se- quences in flow by acting as direct in-line quenching, work-up and purification steps [28,80-85]. Indeed, the scavenger approach to removing excess solution phase reagents or byproducts has had a significant impact on the use and ultimately the successful adoption of flow chemistry into many research laboratories.

Another inherent benefit of flow is the option of safely using solvents at temperatures significantly above of their standard boiling points, increasingly important for process intensification. The implicit high pressure self-contained environment created by a pumped flow system allows for superior reaction kinetics often achieved by elevating the working temperature of the system. This capability also opens up the opportunity for the integration of volatile and gaseous reagents into flow [86]. Due to the inherently low active volumes of reactants that are processed in the reaction zone within a continuous-flow reactor, the safety risks associated with critical event are greatly diminished. In addition, process reliance and sustainability are further improved by adding extra safety monitoring features such as pressure sensors that can both detect, assess, and if necessary, invoke emergency venting or diversion of material to auxiliary depressurisation stages, often without needing a full reactor shut down.

There are numerous examples within the literature in which a flow synthesis has been demonstrated to yield superior results to batch. Higher yields and rates of reaction, and greater selectivities can often be achieved in flow. For example, the rate of the aldol reaction of a silyl enol ether $\mathbf{1 3}$ with 4-bromobenzaldehyde (14) showed a marked increase upon transposition to flow [87]. The reaction that took 24 hours to run to completion in batch, reached $100 \%$ conversion after just 20 minutes in flow (Scheme 1).

The Swern oxidation under trifluoroacetic acid-promoted conditions is often problematic due the formation of side products. In batch, benzaldehyde (17) was formed in 49\% from benzyl 


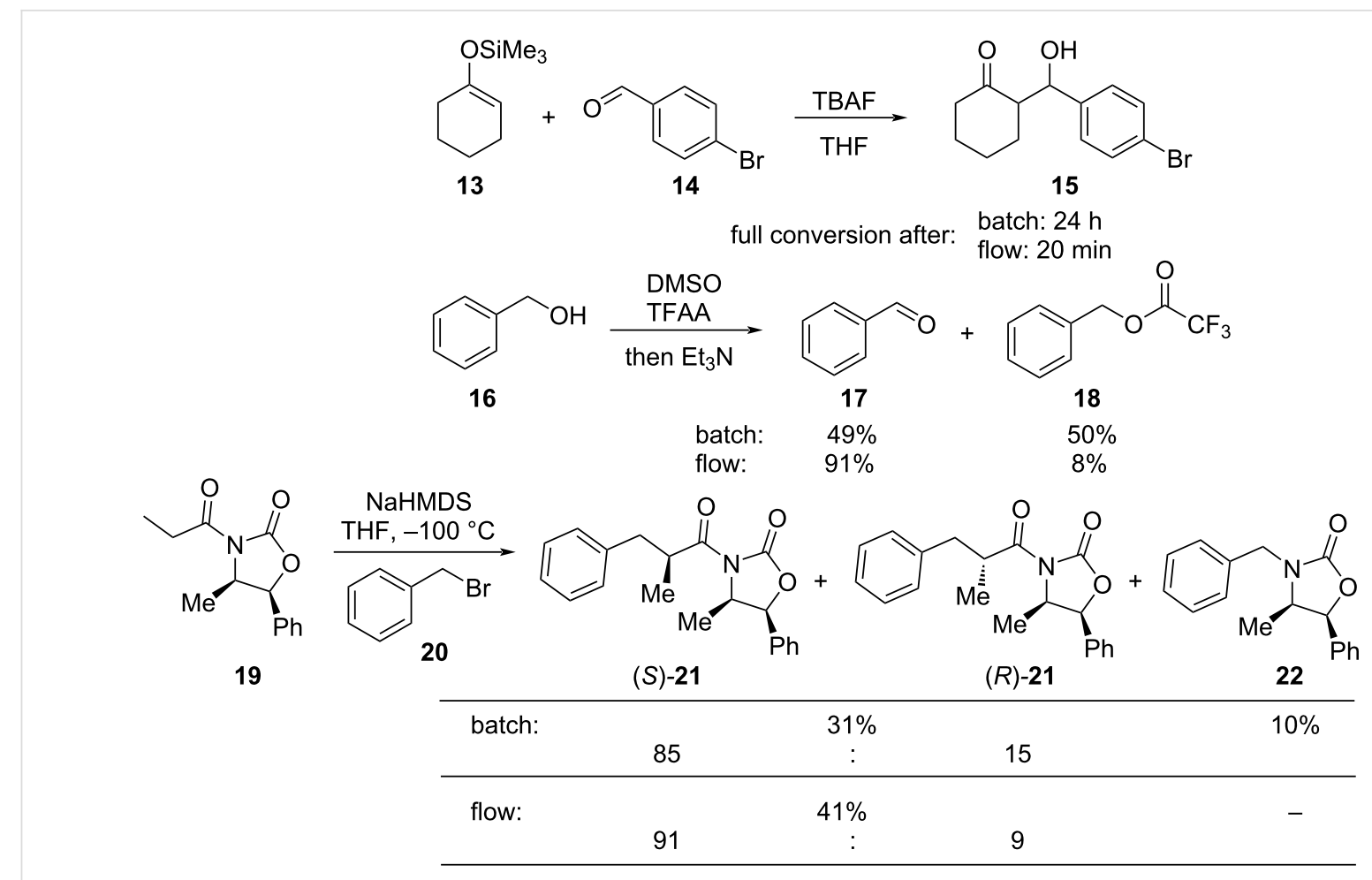

Scheme 1: Examples of reactions for which flow processing outperforms batch.

alcohol (16), alongside $50 \%$ of the trifluoroacetate 18. Carrying out the same process via a flow-based protocol allowed for the formation of benzaldehyde (17) in $91 \%$ yield with only $8 \%$ of the accompanying side product $\mathbf{1 8}$ [88]. Similarly, a stereoselective alkylation of an Evans' type auxiliary yielded superior results in flow [89]. Alkylation of oxazolidinone $\mathbf{1 9}$ with benzyl bromide (20) in batch gave only a combined $31 \%$ yield of the benzylated products $\mathbf{2 1}$, with a $70 \%$ diastereomeric excess (de) accompanied by $10 \%$ decomposition to the $N$-benzyl derivative 22. In flow, however, decomposition was completely avoided and an improved yield of $41 \%$ was achieved with an associated increased of the de to $82 \%$.

Whilst the observation of improved processing control under flow conditions compared to batch is not necessarily always the case, there are many instances in which other advantages can be gained by adopting a flow approach. Many of these improvements are inherently linked to the physical engineering of the flow system which are designed to yield improved mixing, better heat transfer and a more structured and consistent processing environment (equating to constant dosing and mixing rates, defined and reproducible reaction timing and exacting control over all processing parameters). Flow chemistry therefore offers a powerful tool for chemists both from a research perspective and later in terms of manufacturing capacity. This has already been widely demonstrated by the pharmaceutical and agrochemical industries where the uptake of flow chemistry has been significant and widely impactful [30]. In the coming years we will undoubtedly see increasing adoption across the entire field of organic synthesis representing a wider spectrum of related industry sectors.

\section{Review \\ Important transformations for the fragrance industry}

The following section has been divided according to reaction types that are identified as having particular importance to the fragrance and flavours industry. Within each sub-section, an insight into recent developments and the related literature regarding the performance of these reaction types as a continuous-flow process is provided.

\section{Condensation reactions}

A carbonyl functionality is encountered in the vast majority of fragrance ingredients. The ability therefore to manipulate carbonyl moieties within a molecule will forever serve as an important transformation to fragrance chemists. This section investigates the aldol reaction as this is a vital $\mathrm{C}-\mathrm{C}$ and $\mathrm{C}=\mathrm{C}$ bond forming tool within this class of reactions. Other sequences such as the Knoevenagel and Darzens condensation, however, will also be considered as part of the evaluation. 
The condensation of benzaldehyde derivatives, such as $p$-anisaldehyde (23) and cuminic aldehyde (27), with propanaldehyde (24) are important transformations for the preparation of the corresponding anisylpropanal 26, and cyclamen aldehyde (29) (Scheme 2) [90,91].

The syntheses of jasmone derivatives also represent important fragrances whose production involve aldol reactions as key steps. Chapuis et al. have described, several methods for the synthesis of methyl dihydrojasmonate (33) and other $\alpha$-substituted cyclopentanone analogues [92], in addition they also performed $\alpha$-functionalisation of the cyclopentanone ring via an aldol reaction [93]. A common synthesis of dihydrojasmone (35) uses an intramolecular aldol reaction to simultaneously form the cyclopentanone ring and the double bond in the correct positions $[94,95]$.

Aldol reactions are most often exothermic, especially those involving highly reactive aldehydes (e.g., benzaldehydes) and low-molecular-weight enolate nucleophiles, therefore, temperature regulation is vital for selectivity. This requirement for strict temperature control makes aldol reactions highly suited to flow processing conditions. In 2008, Tanaka et al. [96] disclosed several examples of aldol reaction in flow reacting acetone with a wide range of aldehydes. In batch, acetone-based aldol reactions are typically performed under biphasic conditions by slow addition of the aldehyde to an acetone $/ \mathrm{NaOH}$ mixture kept at low temperatures. Upon scale-up, this carries with it problems such as inefficient mixing, difficulty in maintaining the reaction temperature and the occurrence of greater amounts of acetone and aldehyde self-condensation/polymerisation. By adopting a flow protocol and using a Comet X-01 micromixer (with linear scalability), more efficient mixing was attained and less aldehyde self-condensation was evident (Scheme 3).

Within the microreactor acetone was first deprotonated by merging with a $2.5 \mathrm{M}$ sodium hydroxide stream at room tem-
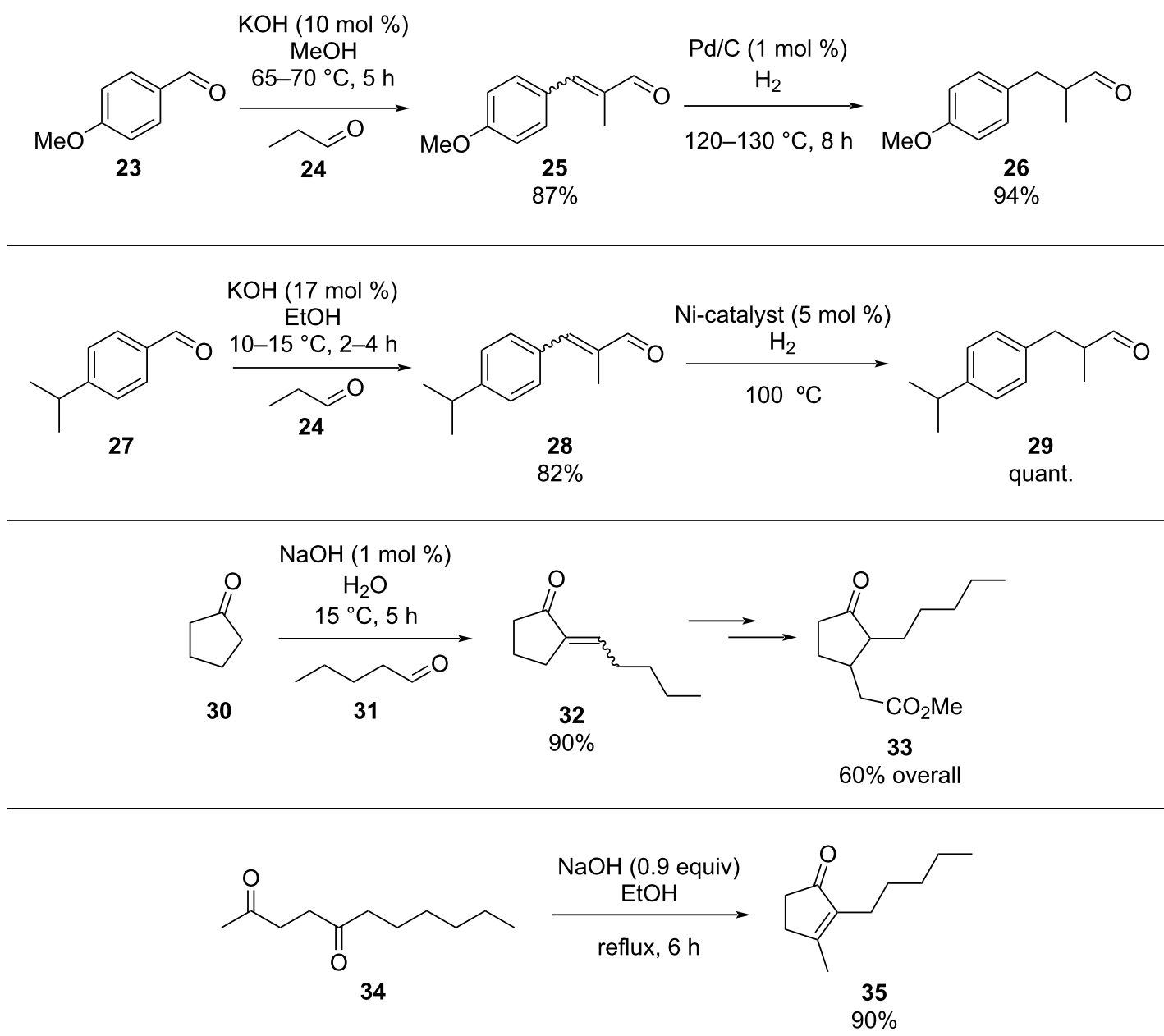

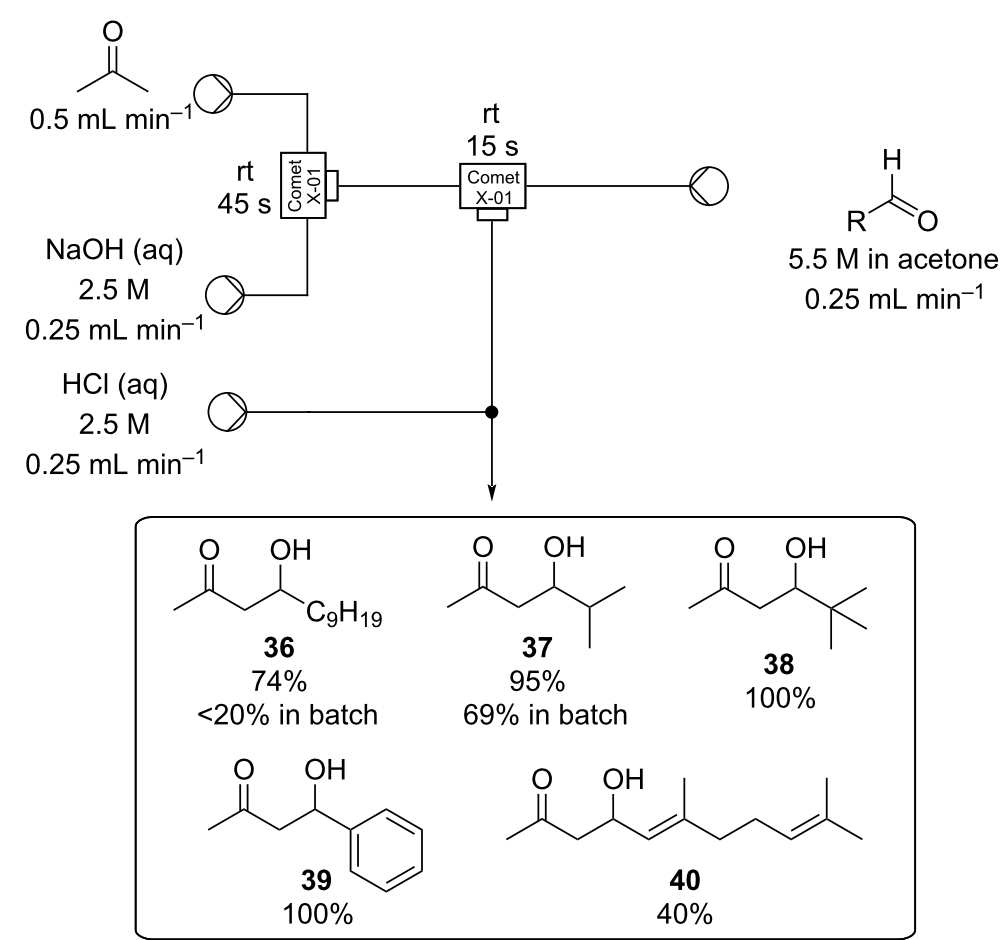

Scheme 3: Biphasic continuous aldol reactions of acetone and various aldehydes.

perature, then, after a residence time of just 45 seconds, the resultant enolate was introduced to a stream containing the aldehyde as a $5.5 \mathrm{M}$ solution also in acetone. The combined flow stream was subsequently quenched after 15 seconds by a downstream inlet of aqueous $\mathrm{HCl}$ afforded the products 36-40. Significantly higher yields were obtained for $\mathbf{3 6}$ and $\mathbf{3 7}$ compared to batch and the methodology developed was later applied to a large-scale synthesis of pristane, an immunoactivating natural product (substrate 37 ) [97,98].

In 2015, the diastereoselective synthesis of (E,S)-3-hydroxy-7tritylthio-4-heptenoic acid 43, a key component of cyclodepsipeptide histone deacetylase (HDAC) inhibitors, was achieved in flow (Scheme 4) [99].<smiles>CC(=O)N1C(=O)OC(c2ccccc2)(c2ccccc2)[C@H]1C(C)C</smiles>

41 $0.13 \mathrm{M}$ in THF $1 \mathrm{~mL} \mathrm{~min}{ }^{-1}$ LiHMDS $0.15 \mathrm{M}$ in THF $1 \mathrm{~mL} \mathrm{\textrm {min } ^ { - 1 }}$

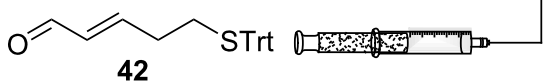
42 $0.05 \mathrm{M}$ in THF $2 \mathrm{~mL} \min ^{-1}$

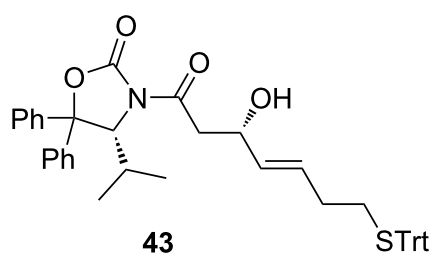

$85 \%, 56 \%$ ee 
Acetyloxazolidinone $\mathbf{4 1}$ was used as a chiral auxiliary which was then removed by hydrolysis in the final step of the overall seven-step synthesis. Deprotonation of $\mathbf{4 1}$ using LiHMDS in THF in a primary reactor was performed at $-40{ }^{\circ} \mathrm{C}$ and telescoped into a second reactor along with a stream containing the trityl-protected aldehyde, 42. The aldol reaction occurred rapidly (residence time, $t_{\mathrm{R}}=7.5 \mathrm{~s}$ ) at $-40{ }^{\circ} \mathrm{C}$ and furnished 43 in $85 \%$ yield, conversion $>95 \%$. Simple syringe pumps and cooled micromixers (Comet X-01) were used at both merging points, allowing the reaction to be conducted in very short timescales. Doi's example also demonstrates the ease with which highly reactive reagents such as lithium bis(trimethylsilyl)amide can be used to effect sensitive aldol reactions in flow.

Another recent example of an aldol reaction on a complex system originates from the group of Gauthier, which details the production of the HIV non-nucleoside reverse transcriptase inhibitor (NNRTI) doravirine (49) in flow (Scheme 5) [100].

The aldol reaction of an aromatic ester $\mathbf{4 4}$ with a vinylogous ester $\mathbf{4 5}$ was achieved in a continuous manner, yielding the hydroxyl adduct, 46 , in $85 \%$ yield within just a 15 second residence time. Here, a slightly different approach was taken in that the two starting materials were combined into a single stream and the base (potassium tert-amyloxide) added via a separate channel. This was presumably established to avoid base-mediated decomposition of the starting material $\mathbf{4 4}$ if prepared as a stock solution. The quenched aldol outlet stream was directed into a cooled receiver vessel, to which trifluoroacetic anhydride (TFAA) was simultaneously added, creating a net semi-continuous process. Subsequent ammonia-mediated cyclisation of the resultant diene followed by alkylation with the chlorotriazolinone 48 yielded doravirine (49) in an overall 52\% yield. The use of an interrupted flow sequence with intermediate batch collection of the output is an easy way of overcoming one of the problematic issues in flow processing; that being assimilating the different residence time requirements in sequential downstream processing. By introducing a batch collection stage this effectively creates a reset on the timing of the individual flow steps. This allows the processing advantages of flow to be utilised for enacting the chemical steps whilst simplifying the timing and connectivity issue. Needless to say, it is inherent to the success of such an approach that there exists at a suitable

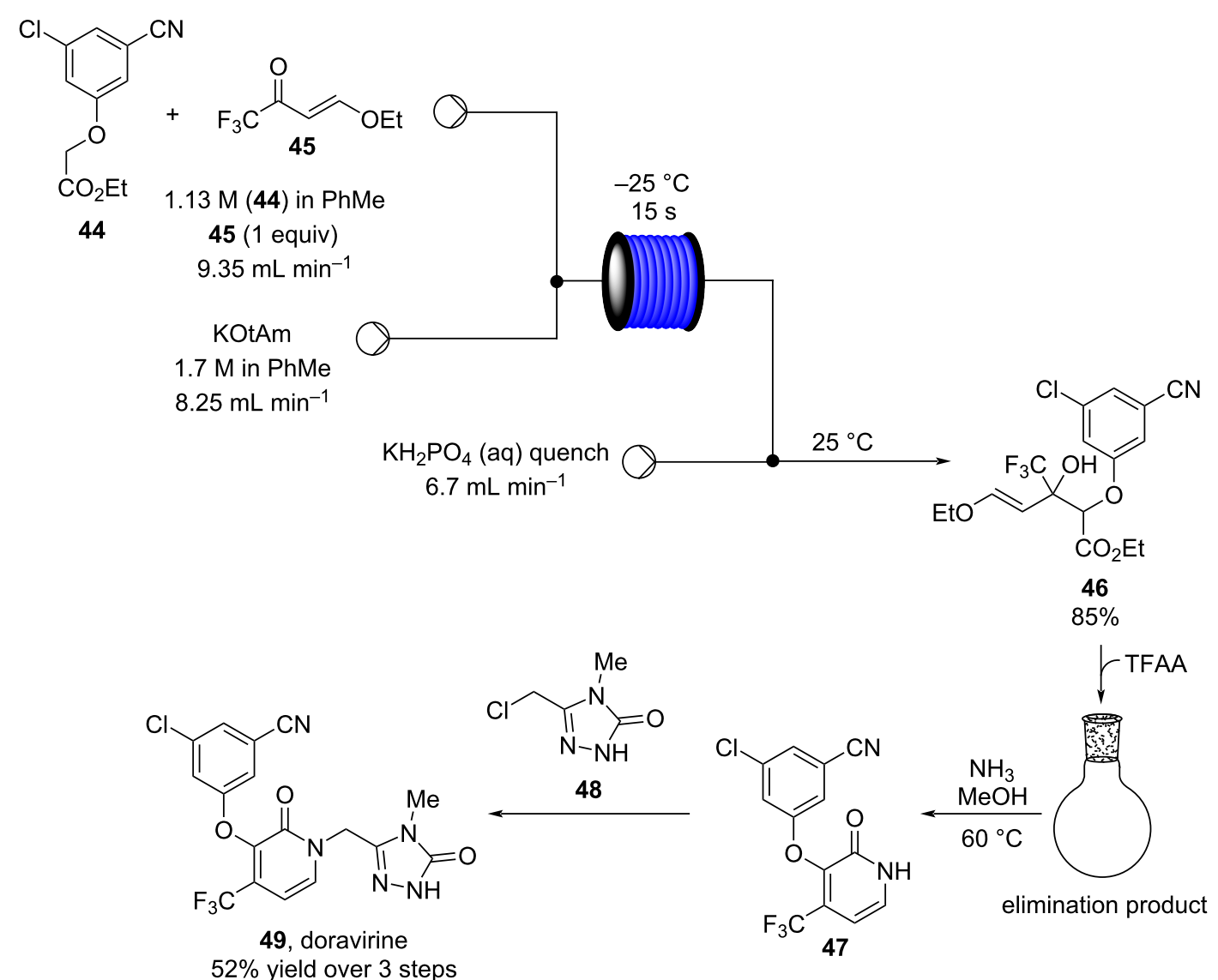

Scheme 5: A semi-continuous synthesis of doravirine (49) involving a key aldol reaction. 
point in the processing sequence a stable collectable intermediate.

Organocatalysed aldol reactions have become an important tool for the synthesis of chiral molecules as it usually employs cheap and naturally available catalysts such as proline. However, a major drawbacks of these process are the long reaction times and high catalyst loading that often need to be used [101,102]

In 2009, Seeberger et al. described a version of an enantioselective aldol reaction in flow using a microreactor (Scheme 6) [103]. The approach allowed for a marked reduction in reaction times ( 20 min vs $40 \mathrm{~h}$ ) via improved mixing, and the ability to conduct the experiment at higher temperature without detrimental results. They also confirmed the easy scalability of the process from a 1 to $4 \mathrm{~mL}$ reactor without loss of selectivity and efficiency. The method was applied on different aldehydes and ketones obtaining moderate-good yields (38-84\%). The results obtained for aldehydes $\mathbf{3 9}$ and $\mathbf{5 6}$ are low as partial dehydration occurs. It was noticed that by reducing the quantity of catalyst less dehydrated product was observed.
A similar approach in aqueous media was optimised by Gröger et al. using a modified proline catalyst. In this example, the authors employed tubular coiled reactors and, to enhance the mixing effect, high flow rates and long tube lengths were applied (Scheme 7). As biocatalysis is becoming more and more important, one of the major issues is the solvent usage. Water is not usually the media of choice to perform organic reactions, therefore, the group of Gröger created a proof-of-concept apparatus capable of subsequently being telescoped directly into a biocatalysed process [104].

An interesting avenue which can be exploited by carrying out aldol processes in flow is the possibility to incorporate heterogeneous solid-supported reagents as catalysts. The ways in which these are used fall into three main categories; monolithic (a), wall-coated (b) and packed bed (c) reactors (Figure 6) [105].

Monolithic columns are prepared by reagent incorporation into a mixture of monomers which are co-polymerised to create a network of micro- and mesoporous channels that fill the entirety<smiles>O=Cc1ccc([N+](=O)[O-])cc1</smiles>

$60^{\circ} \mathrm{C}, 20 \mathrm{~min}$

$1 \mathrm{M}$ in acetone $25 \mu \mathrm{L} \min ^{-1}$

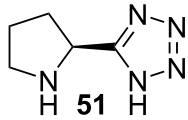

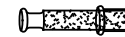

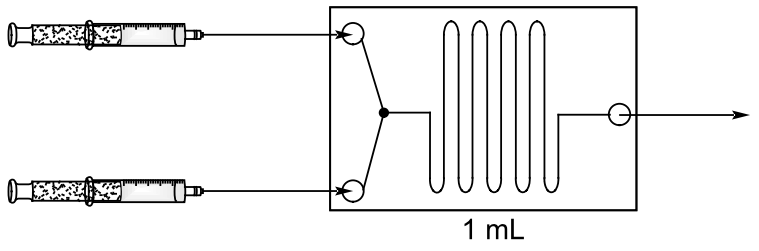<smiles>CC(=O)C[C@@H](O)c1ccc([N+](=O)[O-])cc1</smiles>

$0.05 \mathrm{M}$ in DMSO

$79 \%$ yield

$25 \mu \mathrm{L} \min ^{-1}$

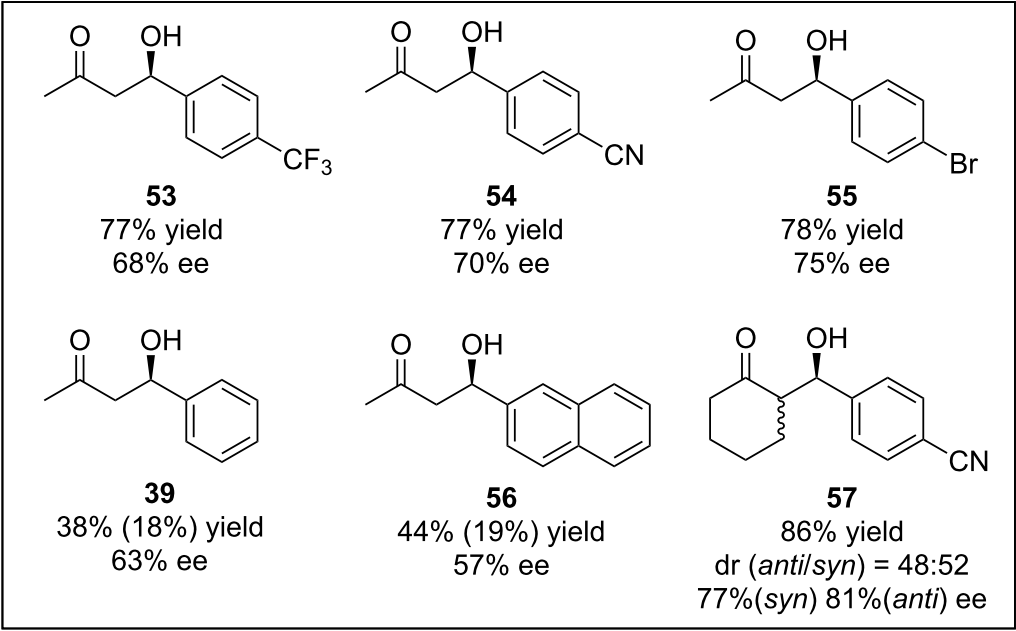




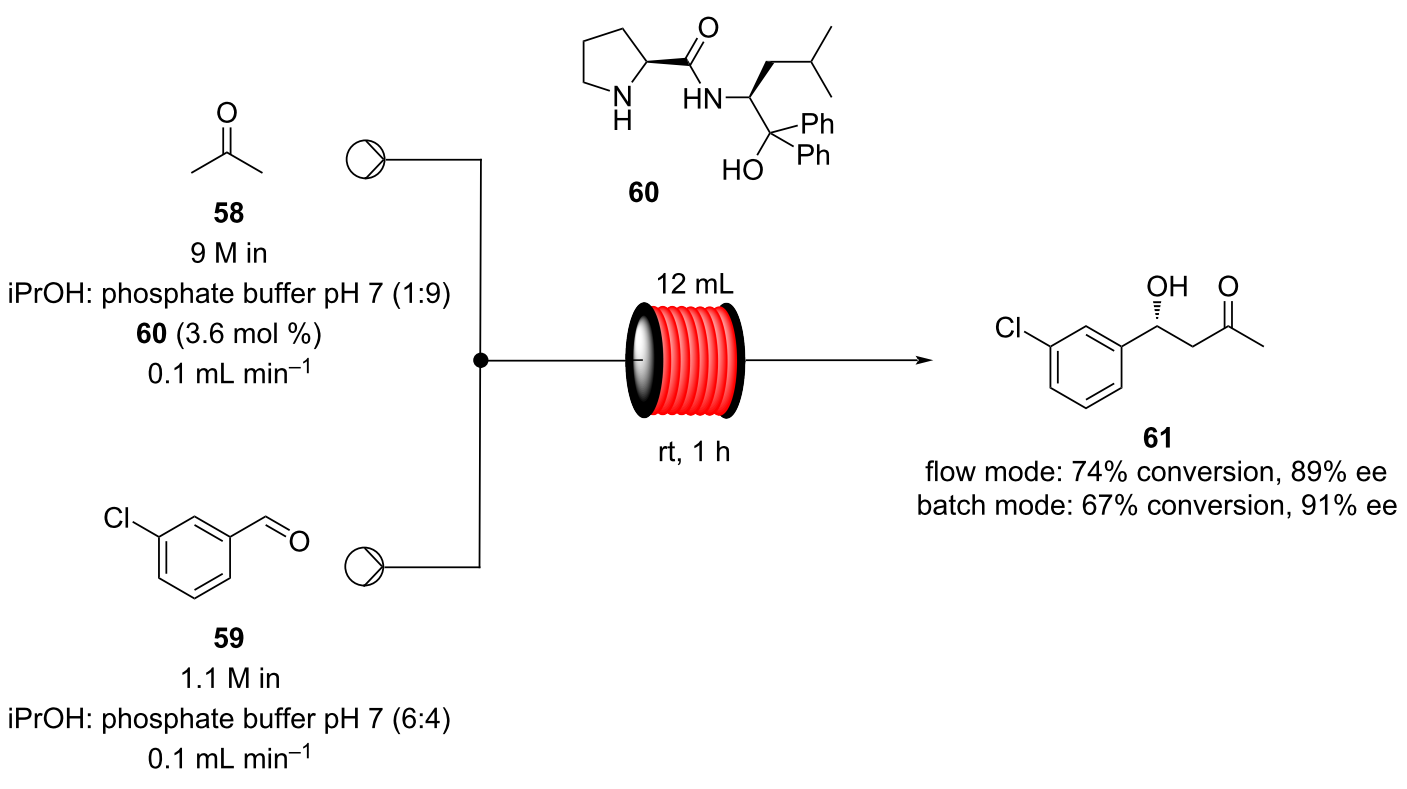

Scheme 7: Gröger's example of asymmetric aldol reaction in aqueous media.

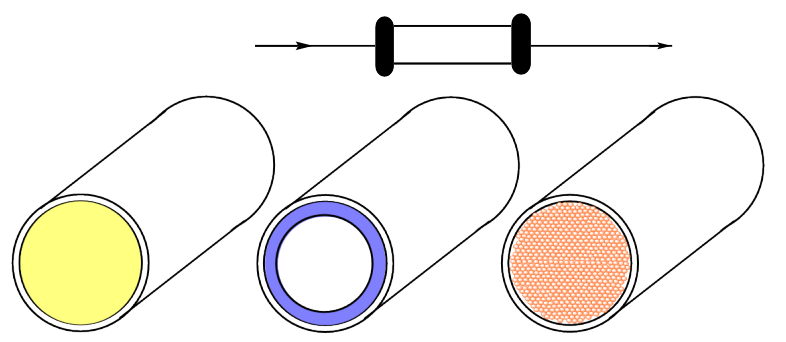

(a) monolith (b) coated-wall

(c) packed bed

Figure 6: Immobilised reagent column reactor types.

of the tube/column. In a coated wall reactor, the reagent is immobilised onto the inner surface of the reactor. Finally, packed bed reactor columns are typically filled with small beads or particles of the reagents tethered to polymeric or inorganic supports.

The use of column reactors carries with it many potential advantages. As mentioned previously, the heterogeneous nature of such a system eliminates the requirement for filtration, neutralisation and often a work-up procedure meaning that this approach is very accommodating of direct telescoping into further reaction steps and reactors. So far, heterogeneous catalysis of aldol reactions in flow has been mostly geared towards achieving transformations with high enantioselectivity, towards decreasing the catalyst expense. As enantioselective catalysts require expensive structural modifications, the possibility of im-

mobilisation would facilitate catalyst recovery/recycle and drastically reduce the cost of the associated process. In 2012, silicasupported 5-(pyrrolidin-2-yl)tetrazole $\mathbf{6 3}$ was used to effect highly selective aldol reactions of simple ketones and aromatic aldehydes in flow [106]. Photoinduced thiol-ene coupling was used to tether the catalyst onto silica particles, yielding an easily handled powder which was packed into a short stainless steel column (Scheme 8).

The resultant packed-bed microreactor was found to possess good long-term stability and could be used for the catalysis of a range of aldol reactions, one example is given in Scheme 8 . Conveniently, just one starting material stock solution was all that was necessary, meaning that 4-chlorobenzaldehyde (64) could be introduced to the reactor alongside cyclohexanone (65) in a single flow stream. A simple HPLC pump, mild heating $\left(50{ }^{\circ} \mathrm{C}\right)$ and a residence time of 25 minutes was all that was required to give the $\beta$-hydroxy ketone $\mathbf{6 6}$ with $>95 \%$ conversion, with modest diastereomeric ratio (dr) of 3:1 and high ee (82\%).

In the same year, the same group developed a polystyrene resinsupported proline, which was confirmed to be highly suitable for continuous-flow setup whose efficiency and selectivity was highly stable over 30 hours of continuous runtime, allowing to isolate roughly 5 grams of pure aldol 68 (Scheme 9) [107]. The activity and robustness of the catalyst allowed reaction at room temperature over long times and enabled continuous preparation of the desired product with no need for downstream purification. 


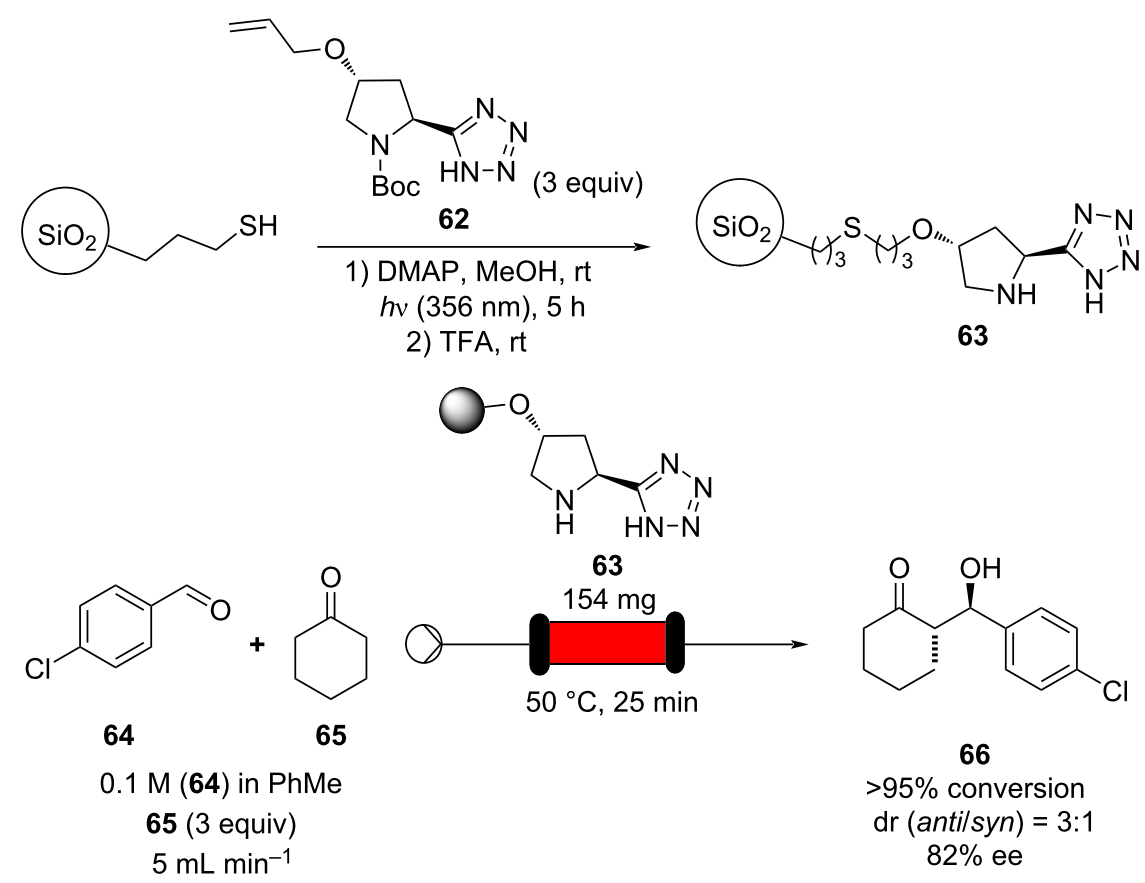

Scheme 8: Photoinduced thiol-ene coupling preparation of silica-supported 5-(pyrrolidin-2-yl)tetrazole 63 and its use in the flow aldol reaction of 4-chlorobenzaldehyde (64) with cyclohexanone (65).

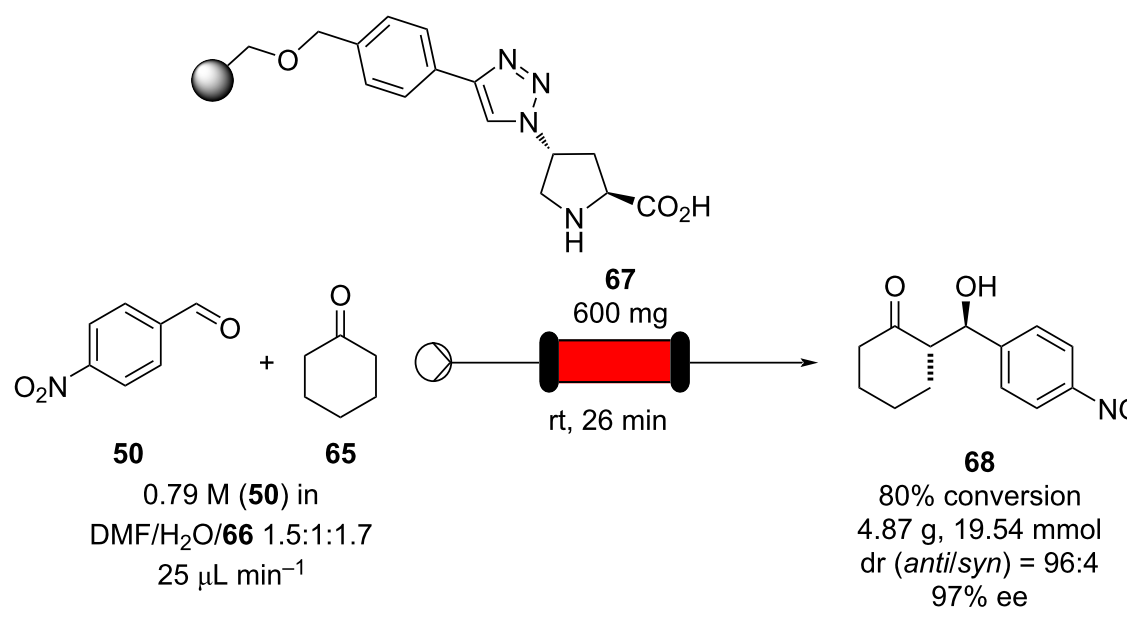

Scheme 9: Continuous-flow approach for enantioselective aldol reactions using the supported catalyst 67.

A similar approach was taken by Ötvös and co-workers in the same year [108]. Asymmetric aldol reactions of aromatic aldehydes with acetone were performed in flow using a solid-supported heterogeneous peptide catalyst. The H-Pro-Pro-Asp-NHresin 69 which was prepared using Fmoc/t-Bu chemistry and assembled onto a TantaGel ${ }^{\circledR}$ polymeric support, then packed into a column reactor (Scheme 10).

Optimisation of the reaction conditions in flow were carried out on $p$-nitrobenzaldehyde (50) and resulted in a $99 \%$ isolated yield of $(S)-\mathbf{5 2}(80 \%$ ee $)$ with a 6 minutes residence time at room temperature. In an analogous batch experiment, 6 hours were required for the reaction to run to completion and achieve a $94 \%$ yield $(78 \%$ ee).

The optimised conditions were then applied to 12 other aromatic substrates and the column was found to be highly robust, giving consistent results over the course of 20 consecutive experiments $\left(20 \times 5 \mathrm{~mL}\right.$ starting material solution $\left(4 \mathrm{mg} \mathrm{mL}^{-1}\right)$, 50 minutes runtime) (Table 5). 


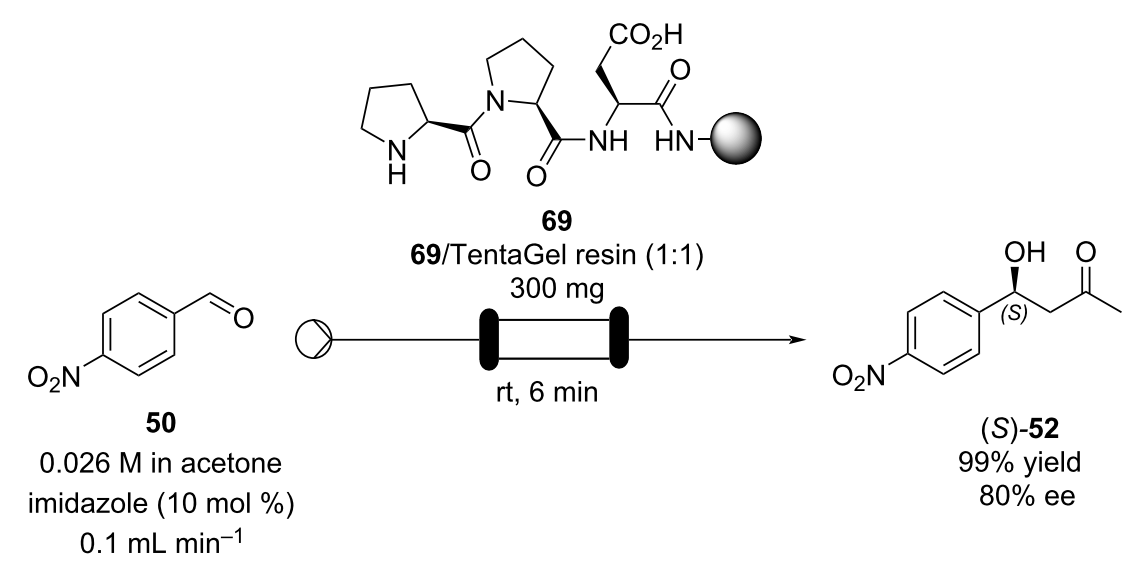

Scheme 10: Ötvös' employment of a solid-supported peptide aldol catalyst in flow.

Table 5: Outcome of multiple consecutive reaction cycles.

\begin{tabular}{lllll} 
Entry & Cycle & $\begin{array}{l}\text { Conversion } \\
(\%)\end{array}$ & $\begin{array}{l}\text { Isolated yield } \\
(\%)\end{array}$ & $\begin{array}{l}\text { ee } \\
(\%)\end{array}$ \\
\hline 1 & $1-5$ & $98-100$ & $97-99$ & $79-80$ \\
2 & $6-10$ & 100 & $97-99$ & $78-81$ \\
3 & $11-15$ & 100 & $>99$ & $79-80$ \\
4 & $16-20$ & $99-100$ & $98-99$ & $79-80$
\end{tabular}

Conveniently, the $(S)$-enantioselectivity could be reversed by inversion of stereochemistry at the terminal proline moiety of the supported peptide. Ötvös' protocol highlights some of the key advantages of this immobilisation approach, i.e., improved reaction kinetics over batch (higher effective catalyst loading), column robustness/ease of recyclability and the facile nature of reaction condition and substrate screening.

To increase the greenness and reduce the consumption of the catalyst, Yamamoto and Nakashima have recently developed a proline tetrazole $\mathbf{5 1}$ packed-bed reactor system, exploiting the low solubility of the catalyst in less polar solvents (Scheme 11). Thus, a stream of 2,6-dichlorobenzaldehyde (70) and cyclohexanone (65) along with $10 \mathrm{~mol} \%$ of AcOEt and $3 \mathrm{~mol} \%$ $\mathrm{H}_{2} \mathrm{O}$ were directed as a flow stream into the loaded column to partake in the aldol reaction to furnish adduct $\mathbf{7 1}$ in $95 \%$ yield and excellent $\mathrm{dr}$ and $92 \%$ ee. The system allowed a sharp decrease in the loading of the catalyst to $0.052 \mathrm{mg}$ during a 5 mmol scale production run [109].

Yamamoto and Nakashima's approach is a particularly simple strategy that demonstrates the ease and creativity that can be used in setting up an efficient flow system without the need for supports, hence only modifying the solubility of the catalyst.

However, in the above-described reactors the main drawbacks presented were the high pressure drop along the column and the poor handling of solid particles. This resulted in a reduced reliability especially during scale-up equating to prolonged run times. As a result, alternative support formats have been investi-

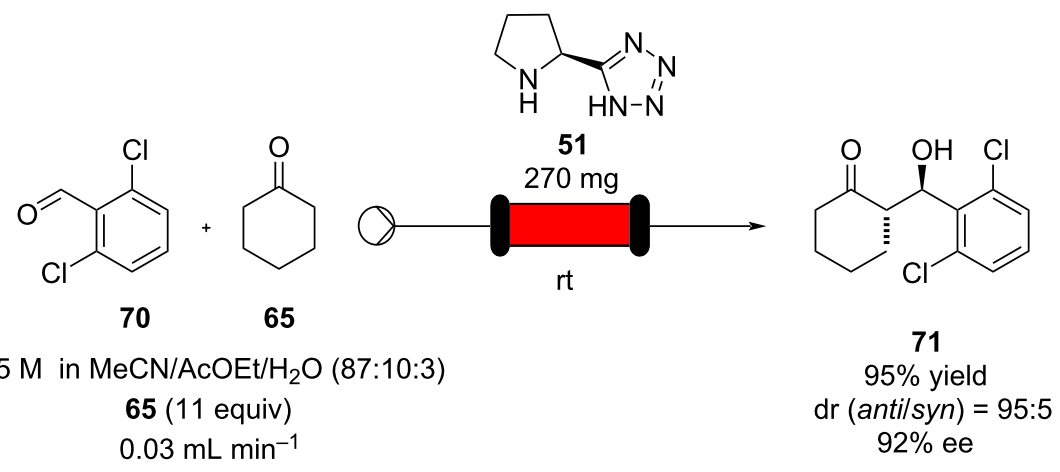

Scheme 11: The use of proline tetrazole packed in a column for aldol reaction between cyclohexanone (65) and 2,6-dichlorobenzaldehyde (70). 
gated. In this context there has been a growing interest in the use of functionalised hollow fibres including their application as membranes for gas separation [110] (e.g., $\mathrm{CO}_{2}$ absorption [111]) as they offer the advantage of high surface-to-volume ratio and scalability. These systems consist of nanoparticles embedded into a porous polymer matrix. In heterogeneous catalysis, a higher particle stability and good distribution of the catalyst leads to a reduction in pressure drop alongside a stronger interaction with the reagents. As a result, several hollow fibresimmobilised catalysts have been prepared and applied in flow scenarios [112]. A titania-, zirconia- and silica-implanted polyamide-imide (PAI) hollow fibre was grafted with aminosilane functional groups in order to create a bifunctional catalyst for continuous-flow aldol condensations. A solution of the reagents was directed into the stainless steel module, containing a series of five hollow fibres, perpendicular to the fibre alignment. The solution permeates the fibres and is pushed through the reactor using a nitrogen flow stream (Scheme 12) [113].

The reactor output reached a maximum conversion of $64 \%$, generating a 1:1 ratio of the aldol adduct 39 and the further condensation product 72. It was also shown that the selectivity for 39 could be improved by increase the flow rate, although this also coincided with a lower conversion of just $13 \%$. Nitroaldol reactions were also performed and nitrostyrene (74) was achieved with $54 \%$ selectivity $(1: 1 \mathbf{7 3} / \mathbf{7 4})$ and $100 \%$ conversion at $90{ }^{\circ} \mathrm{C}$. It is worth noting that the degree of production could be readily increased by numbering up of the hollow fibres which are bundled in the column.
Other examples of the use of column reactors for the catalysis of aldol reactions include: The use of immobilised aldolase enzymes for the synthesis of carbohydrates [114] and the use of a calcinated hydrolactite-packed column for the condensation of furfural with acetone [115], however, this still remains a relatively under-explored area of research. While the majority of reported examples have been of academic origin, there is a high level of interest in the pursuit of aldol reactions in flow by industry. The production of $\alpha, \beta$-unsaturated $\mathrm{C}_{10}$-aldehydes [116] as multiphase aldol condensations in flow was also patented in 2014 by Strutz et al. [117].

Microinnova Engineering has devised a mesofluidic platform for continuous aldol reactions used in the synthesis of nabumetone (77) and other 4-aryl-2-butanone derivatives demonstrating the ease of scale-up associated with such transformations in flow [118]. The condensation of a range of aromatic aldehydes with acetone were again studied, with $p$-anisaldehyde (23) being used for scale-up. A simple reactor design based upon Teflon ${ }^{\circledR}$ tubing ( $8 \mathrm{~mm}$ inner diameter) was used in conjunction with static mixing elements as shown in Scheme 13, allowing for a flow rate of $45 \mathrm{~mL} \mathrm{~min}^{-1}$ to be used. A 66 second residence time gave full conversion to the $\alpha, \beta$ unsaturated product $\mathbf{7 8}$ in high isolated yield, generating a calculated theoretical throughput of $0.35 \mathrm{~kg}$ of product per hour. The authors claim that, in principle, by simply elongating the reactor by a factor of 5 and increasing proportionally the flow rate a theoretical throughput of $1.75 \mathrm{~kg} \mathrm{~h}^{-1}$ of $\mathbf{7 8}$ would be possible. Although such calculations can be made implementing the necessary changes are not as linearly scalable or

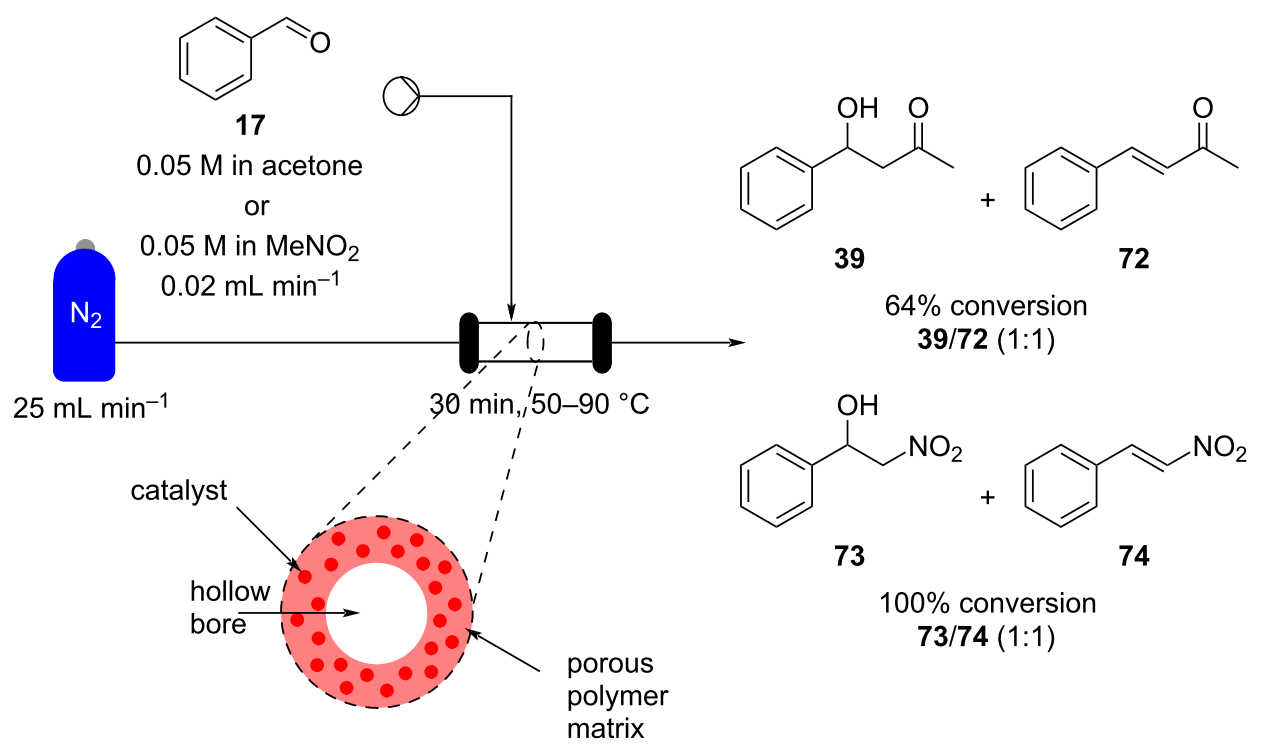

Scheme 12: Schematic diagram of an aminosilane-grafted Si-Zr-Ti/PAI-HF reactor for continuous-flow aldol and nitroaldol condensation of benzaldehyde (17) with acetone. 


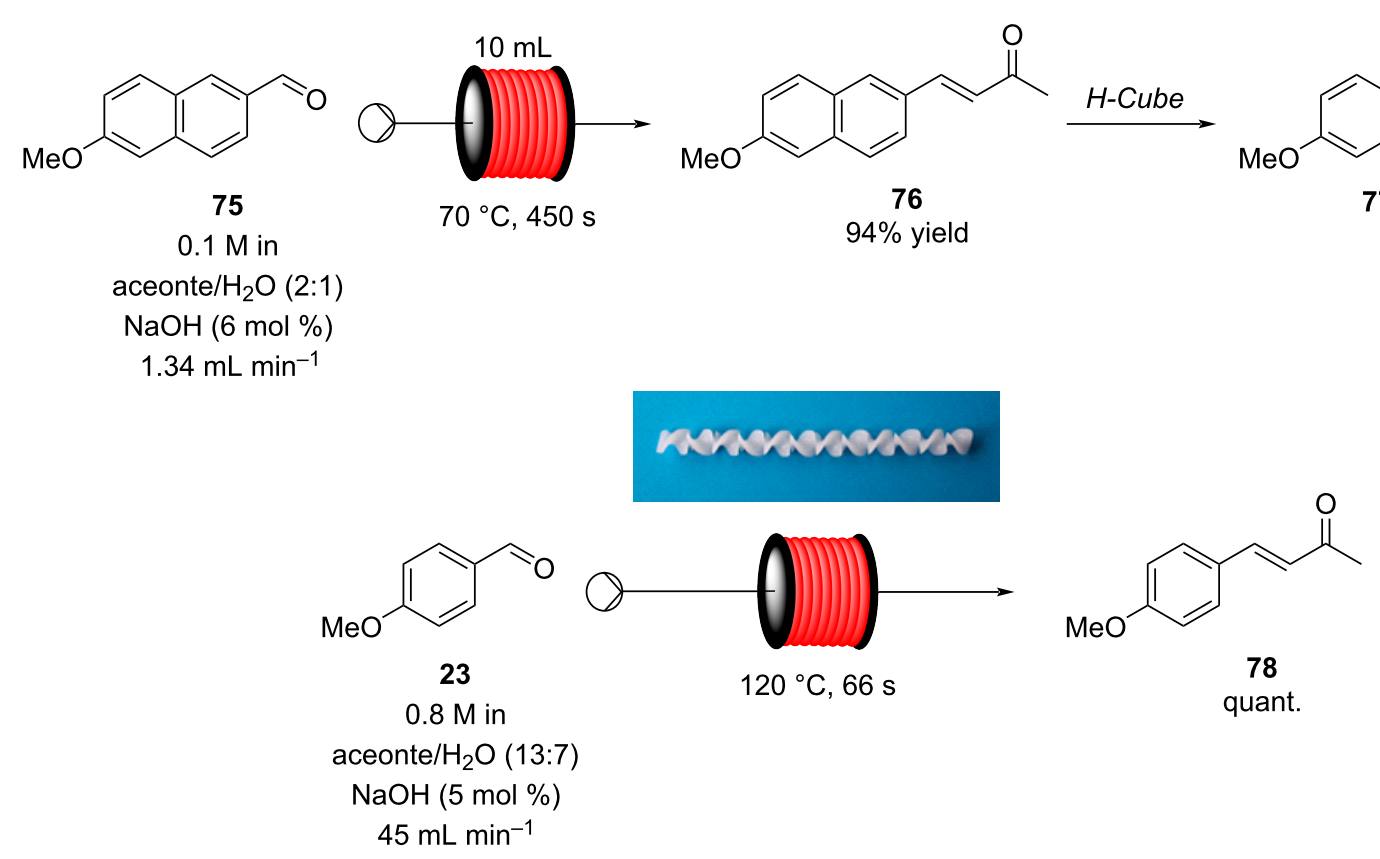

Scheme 13: Continuous-flow condensation for the synthesis of the intermediate $\mathbf{7 6}$ to nabumetone (77) and Microinnova mesofluidic scale-up protocol for the synthesis of 78 [118]. Adapted with permission from [118]. Copyright 2011 American Chemical Society.

simple as implied due to change in the mixing efficiency of the reactor as well as effects of back mixing and increased pressure drop, indeed the efficiency of pumps must also be taken into account. The consideration of the pumping device is a critical aspect of each flow process but is often relegated to simple supporting information. However, each pumping unit has by design a specific range of operation and often a very specific window of optimal pumping efficiency (flow rate, pressure drop and viscosity) within this domain. Hence, especially when considering the concepts of direct scale-up this becomes an increasingly important aspect.

Examples of flow systems applied on terpenoids are abundant in the literature. As terpenoids are one of the most important odorants in the $\mathrm{F} \& \mathrm{~F}$ industry.
Schütz et al. developed an aldol condensation of citral (79, 63:37 mixture of geranial $(Z)-79 /$ neral $(E)$-79) with acetone to prepare $\psi$-Ionone or pseudoionone (80) in good yield (60-70\%) [119]. By using ion-exchange resins such as Amberlyst ${ }^{\circledR}$ A26 $(\mathrm{OH}$ form) as a heterogeneous base in a fixed-bed reactor, the $\alpha, \beta$-unsaturated compound $\mathbf{8 0}$ was obtained and with a stable performance over more than 15 hours of continuous operation (Scheme 14).

In 2019, the group of Kobayashi reoptimised the Schürt's process for a more general library of molecules (28 compounds were screened) and they managed to overcome the catalyst deactivation enabling a process to be operated continuously for more than 5 days $\left(\mathrm{TOF}=0.09 \mathrm{~h}^{-1}\right.$ and STY $=354 \mathrm{~g}\left(\mathrm{~L} \mathrm{day}^{-1}\right)$ using a mixture toluene/EtOH 9:1, noticing that the presence of

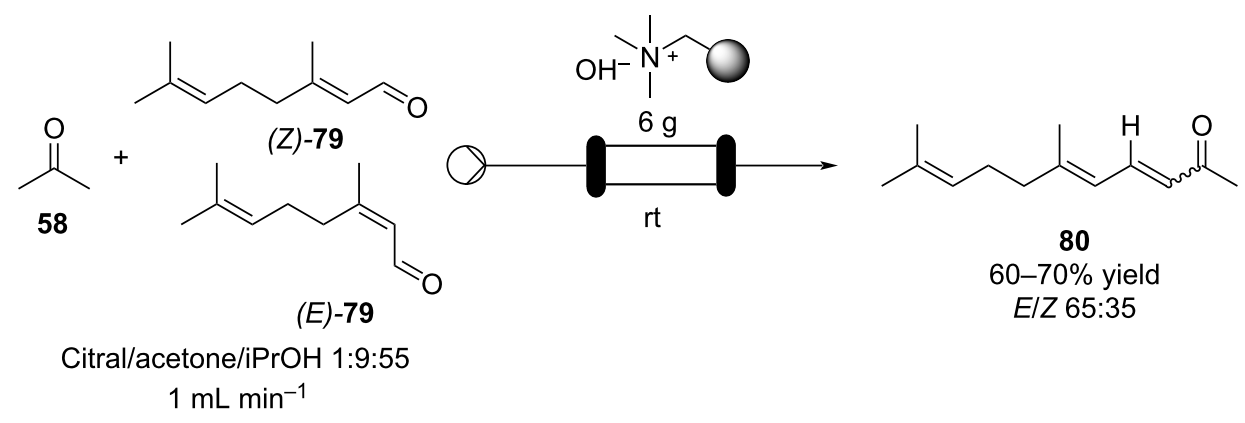

Scheme 14: Synthesis of $\psi$-lonone (80) in continuous-flow via aldol condensation between citral (79) and acetone (58). 
EtOH drastically increased the catalyst's activity. A two-step continuous synthesis was also described for the synthesis of donepezil, a top 100 best-selling anti-Alzheimer's drug [120].

A different approach for the synthesis of $\psi$-Ionone (80) was described by Chen et al., whose group developed an efficient homogenous $\mathrm{NaOH}$-catalysed condensation of citral (79) with acetone using microreactors. The setup allowed the preparation of $\mathbf{8 0}$ in $93.8 \%$ yield with a throughput of $5.24 \mathrm{~g} \mathrm{~h}^{-1}$ [121]. The $\mathrm{NaOH}$ aqueous solution was introduced as a mixture with EtOH, which was discovered to notably increase the mass transfer (thus improving yields from $87 \%$ to $93.8 \%$ ). The authors also compared their apparatus with a tubular version de- scribed by Dobler et al. claiming a higher yield (93.8 vs $86 \%$ ) and a faster reaction time (98.6 s vs $120 \mathrm{~s})$ [122].

In the same patent, Dobler et al. described a process for the synthesis of $\beta$-methylionones (83) in flow. Tubular reactors were employed for the preparation and the two steps were reported separately (Scheme 15). The $\mathrm{NaOH}$-catalysed condensation is performed in a $160 \mathrm{~L}$ tubular reactor with a 4 minutes residence time at $132{ }^{\circ} \mathrm{C}$. The initial reaction mixture is biphasic, and a preliminary separation is carried out before entering the reactor coil. After discontinuous purification procedures, the product $\mathbf{8 2}$ was yielded in $98 \%$ purity ( $72 \%$ yield). The second step, instead, is an acid-catalysed cyclisation which employs the

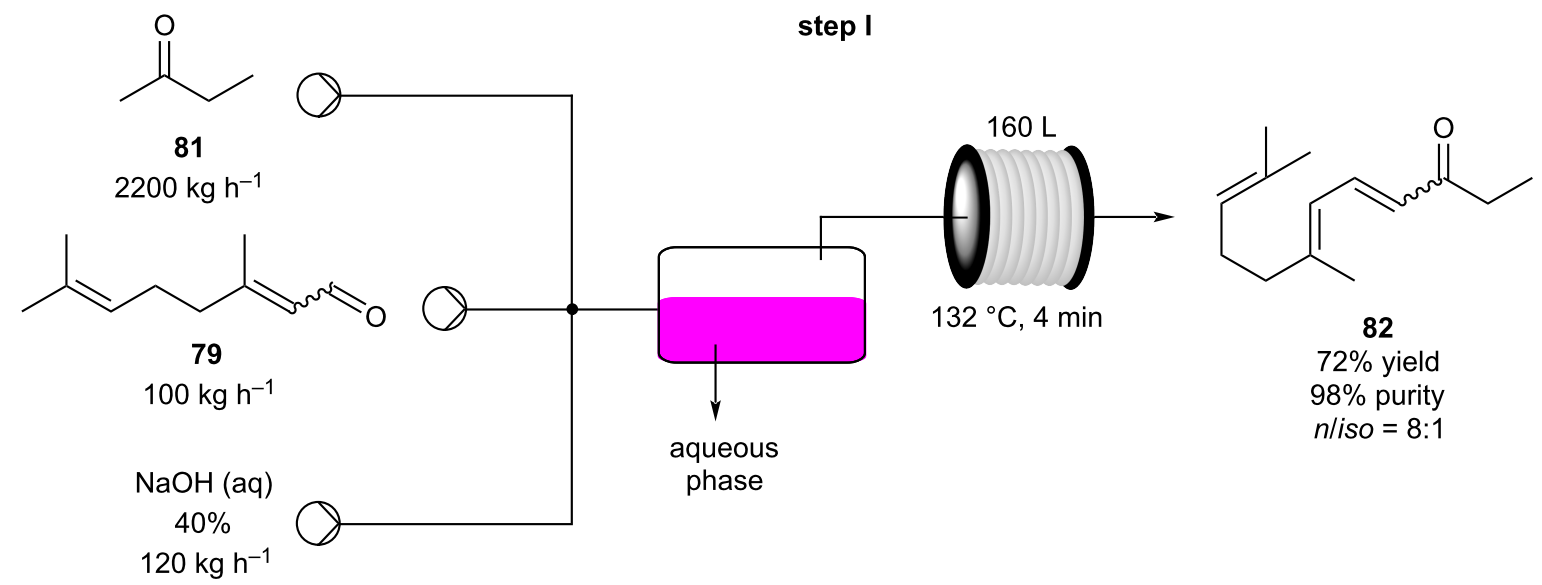

step II

hexane

(cooled at $-8^{\circ} \mathrm{C}$ )

$400 \mathrm{~L} \mathrm{~h}^{-1}$<smiles>CCC(=O)/C=C/C=C(/C)CCC=C(C)C</smiles>

\section{O}

$140 \mathrm{~L} \mathrm{~h}^{-1}$

$\mathrm{H}_{2} \mathrm{SO}_{4}(\mathrm{aq})$

$89 \%$

$200 \mathrm{~L} \mathrm{~h}^{-1}$
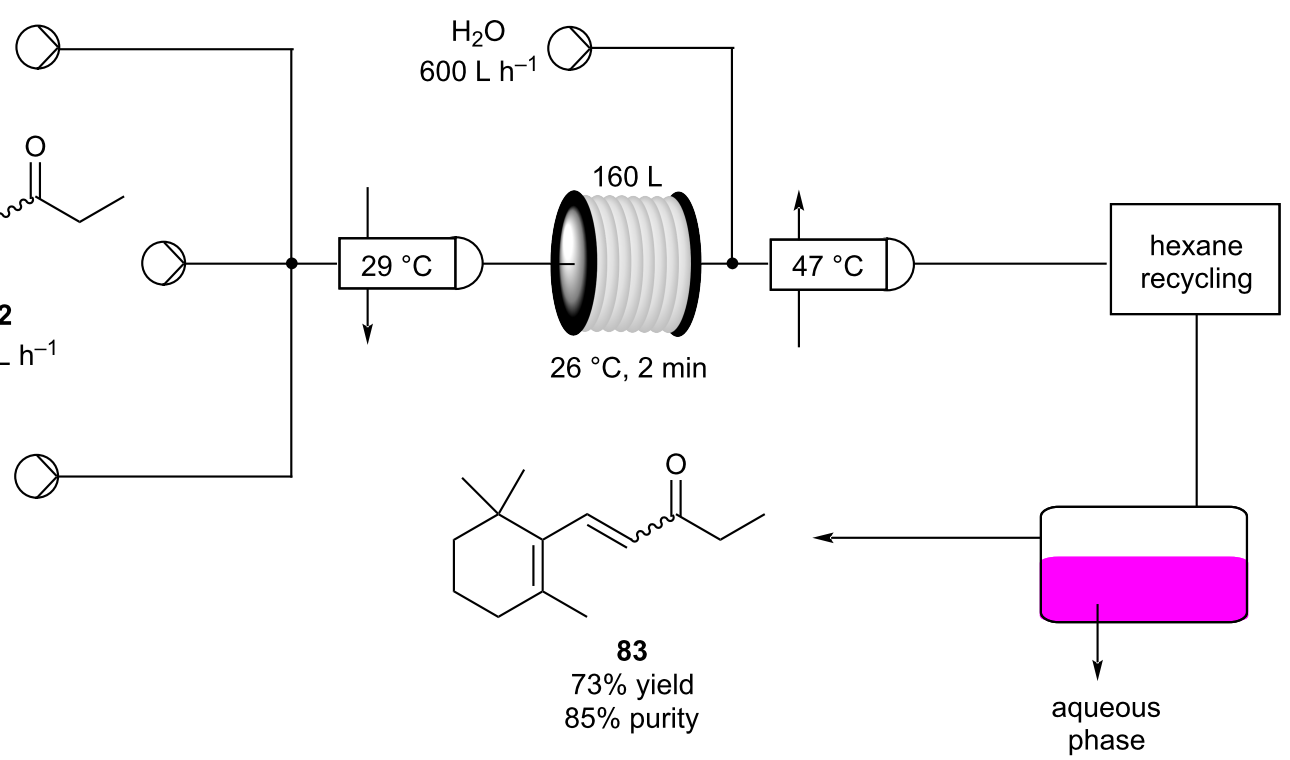

Scheme 15: Synthesis of $\beta$-methyl-ionones (83) from citral (79) in flow. The steps are separately described, and no telescope process was claimed. 
same tubular reactor at lower temperature $\left(26^{\circ} \mathrm{C}\right)$ and reaction time ( 2 minutes). The cooled solution of $\mathbf{8 2}$ in hexane is mixed in flow with sulfuric acid and then heated at $29{ }^{\circ} \mathrm{C}$. After the cyclisation, the stream was quenched with water whilst the temperature was maintained around $45{ }^{\circ} \mathrm{C}$. The hexane was then removed using a countercurrent system with the steam being recycled back through the reactor. After phase separation, the obtained mixture was enriched to $85 \%$ of $\mathbf{8 3}$ (equating to a $73 \%$ isolated yield).

Once the reaction conditions were optimised, the apparatus can merge with other flow systems to develop a parallel continuous multistep process. A recent example, Gavriilidis et al. described a heterogeneous version for the synthesis of 4-(4methoxyphenyl)butan-2-one (85) starting from 4-methoxybenzyl alcohol (84). In this case, the preparation is divided in 3 stages: oxidation, aldol condensation, and reduction (Scheme 16). The setup employs silicon-glass and tubular reactors packed with nanoparticle supported catalysts. The apparatus allows the desired material to be prepared in $48 \%$ yield. The authors pointed out better outcomes were obtained in batch using a MgO-based catalyst, whose usage proved troublesome in flow due to clogging of the reactor channels [123]. This article undoubtedly shows the capacity of flow systems in being easily telescoped for multistep syntheses.
A self-condensation for the synthesis of 2-methylpentenal (86) was also developed by the group of Poliakoff (Scheme 17). The system exploits a sulfonic acid resin (Amberlyst $\left.{ }^{\circledR} 15\right)$ in $\mathrm{scCO}_{2}$ as the solvent system.

The continuous-flow apparatus had an in-line GC for faster screening of reaction conditions. The optimised setup allows to continuously transform propanal (24) in the corresponding selfcondensed material 86 in $43 \%$ conversion for up to 65 hours. They also proved the possibility to perform an in situ hydrogenation of 86 by mixing the resin with a Pd catalyst $(50 \%$ Amberlyst ${ }^{\circledR} 15 / 2 \%$ Pd on silica/alumina). The desired material was indeed obtained with high yields (up to $80 \%$ ) and selectivity (up to 95\%) [124].

In 2013, a continuous-flow multistep synthesis of coumarin (90) was also reported, which involved an intramolecular aldol-type condensation of the acetylated intermediate $\mathbf{8 9}$ derived from salicylaldehyde (88, Scheme 18) [125]. The initial $O$-acetylation step was telescoped directly into the next reactor; the twostep process gave an overall yield of $91 \%$ under optimal conditions. Beneficially as all components were liquids the need for a solvent was completely avoided; from a green chemistry standpoint this is highly attractive and the intrinsic low melting points of many fragrance ingredients and precursors lend them-

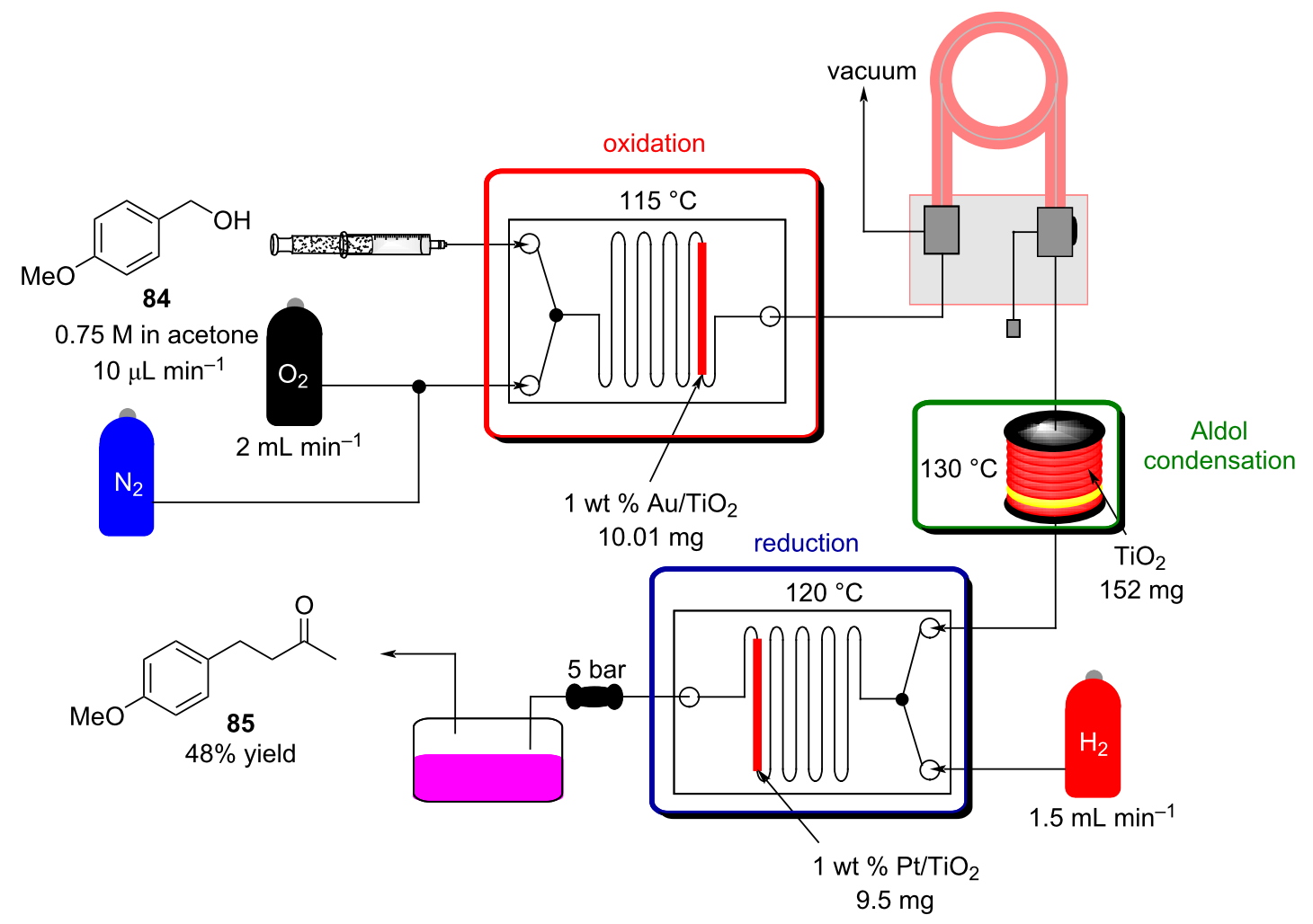

Scheme 16: Continuous-flow synthesis of 85 from 84 described by Gavriilidis et al. 

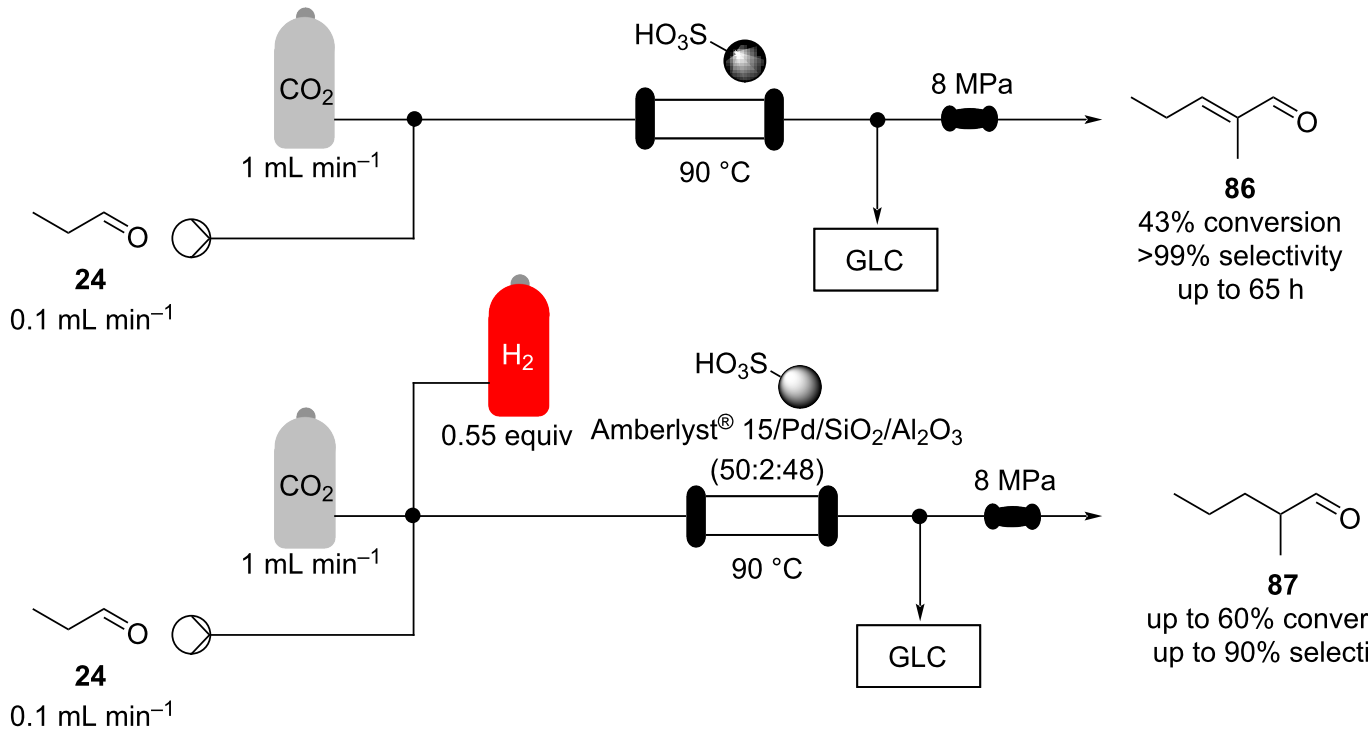

up to $60 \%$ conversion up to $90 \%$ selectivity

Scheme 17: Continuous-flow scCO2 apparatus for the synthesis of 2-methylpentanal (87) and the self-condensed using Amberlyst ${ }^{\circledR} 15$ as a solid catalyst.

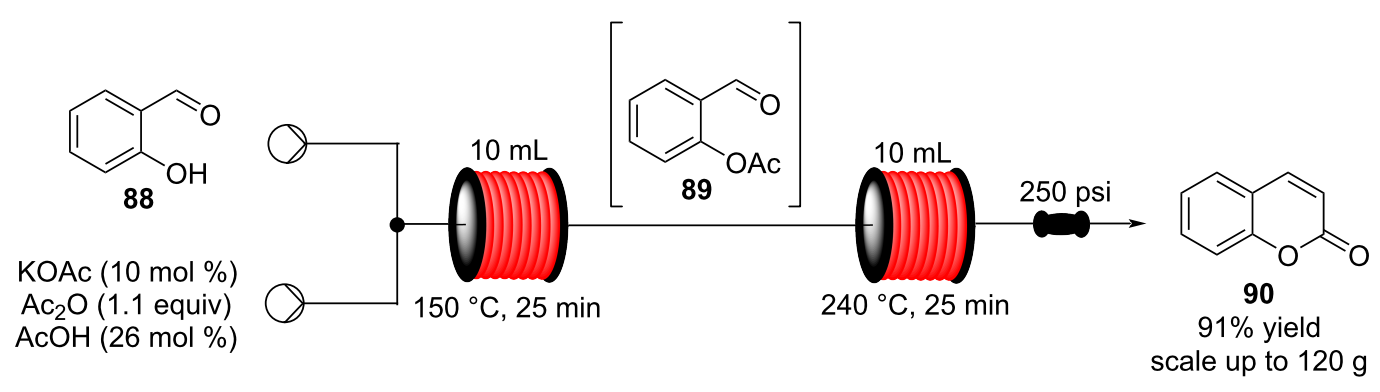

Scheme 18: Chen's two-step flow synthesis of coumarin (90).

selves to continuous-flow approaches in this regard. To show the practicability of the process, a $0.12 \mathrm{~kg}$ scale-up operation was also performed.

A Pechmann condensation in flow for the synthesis of 7-hydroxycoumarin (93) was also reported in 2012 (Scheme 19). The single-step process proved highly efficient forming the desired material in essentially quantitative conversion within 1 hour of processing [126]. The flow output was connected to other apparatus to create a continuous multistep process for the preparation of the 8-formyl-7-hydroxycoumarin (94).

The nitroaldol condensation (Henry reaction) is another important direct $\mathrm{C}-\mathrm{C}$ and $\mathrm{C}=\mathrm{C}$ bond-forming tool which has been widely described in the literature to prepare a range of different valuable intermediates [127-131]. Applications in the F\&F industry are sparse which is probably due to the unpredictable nature of some nitro intermediates. However, late in 1983, Ballini et al. represented the potentiality of the reaction for the synthesis of dihydrojasmone derivatives (Scheme 20) [132].

Flow chemistry embodies the safest conditions for handling hazardous chemicals, therefore opening new opportunities for nitro compounds to be industrially employed.

In 2008, the group of Sartori developed a flow setup for nitroaldol condensations of different benzaldehydes exploiting a silica-supported amine (KG-60- $\mathrm{NH}_{2}$ ) as a heterogeneous catalyst (Scheme 21) [133]. Secondary and tertiary amine-supported catalysts were also investigated, although they were found less active than the primary amine. The authors suggest the reaction occurs through an imine/iminium intermediate as they confirmed a first order relationship between the reaction 
<smiles>CC(=O)CCC(=O)[OH2+]</smiles><smiles>Oc1cccc(O)c1</smiles>

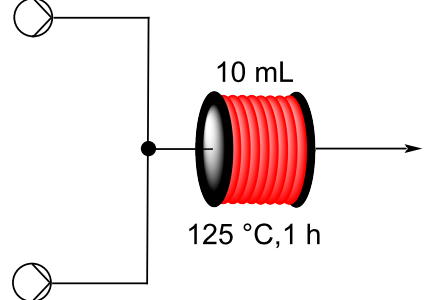<smiles>O=c1ccc2ccc(O)cc2o1</smiles><smiles>O=Cc1c(O)ccc2ccc(=O)oc12</smiles>

$99 \%$ conversion

$0.96 \mathrm{M}$ in $\mathrm{AcOH}$

$\mathrm{HCl} 37 \%$ (0.04 equiv)

$83 \mu \mathrm{L} \mathrm{min}{ }^{-1}$

Scheme 19: Pechmann condensation for the synthesis of 7-hydroxyxcoumarin (93) in flow. The setup extended to comprise 3 additional steps to prepare aldehyde $\mathbf{9 4}$.

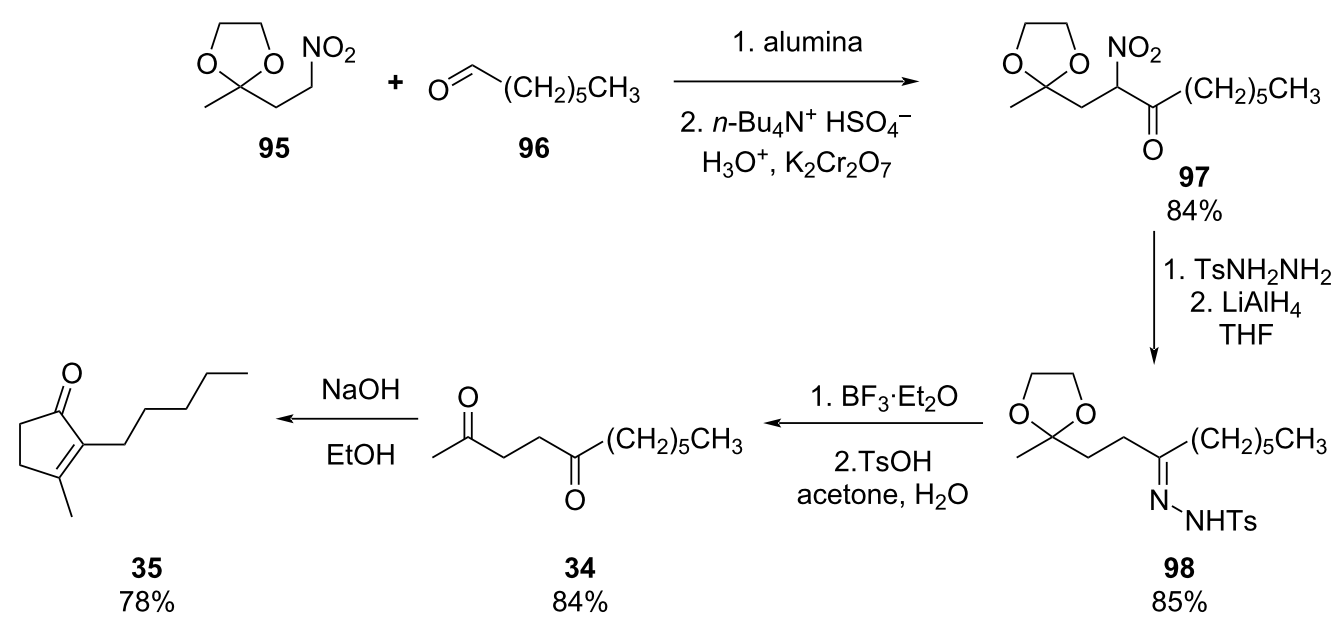

Scheme 20: Synthesis of the dihydrojasmonate 35 exploiting nitro derivative proposed by Ballini et al.
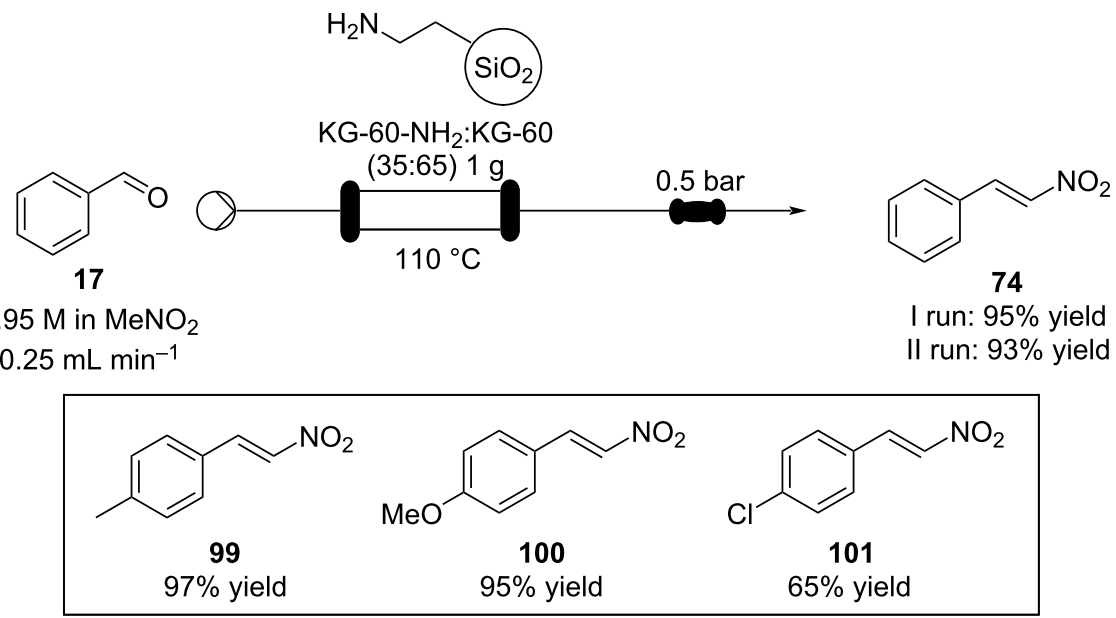

Scheme 21: Silica-supported amines as heterogeneous catalyst for nitroaldol condensation in flow. 
rate and the catalyst concentration. After washing with nitromethane, the catalyst could be reused a second time, obtaining comparable outcomes.

A similar system was later described by Asefa et al. The authors packed a jacketed glass capillary microreactor with an aminefunctionalised mesoporous silica (AP-T) pressed into small pellets (Scheme 22) [134].

The apparatus enabled the preparation of $p$-hydroxy- $\beta$-nitrostyrene (103) in good conversion and selectivity. Its synthesis was revealed to be highly dependent on the residence time as lower flow rates brought about the secondary Michael reaction adduct 104. A secondary amine-functionalised silica was also investigated, although yielding the product in lower selectivity
The optimised setup was shown to be reasonably stable for continuous runtimes of up to 100 hours.

In the last few years, polyacrylonitrile fibres (PANF) along with other synthetic textiles have seized attention in heterogeneous catalysis as supports thanks to their incomparable mechanical properties and low-cost [135]. These fibres are also suitable for flow systems as they can be easily shaped into the reactors. As an example, Tao's group optimised several reactions in batch exploiting a quaternary ammonium functionalised PANF as catalyst $\left(\mathrm{PAN}_{\mathrm{QAB}-8} \mathrm{~F}\right)$. They also reported the Henry reaction of 4-chlorobenzaldehyde (64) in flow (Scheme 23) [136]. The catalyst enables the use of water as the solvent; however, in the flow setup, an organic solvent was needed to form a monophasic solution. The apparatus shows high stability $(86 \%$

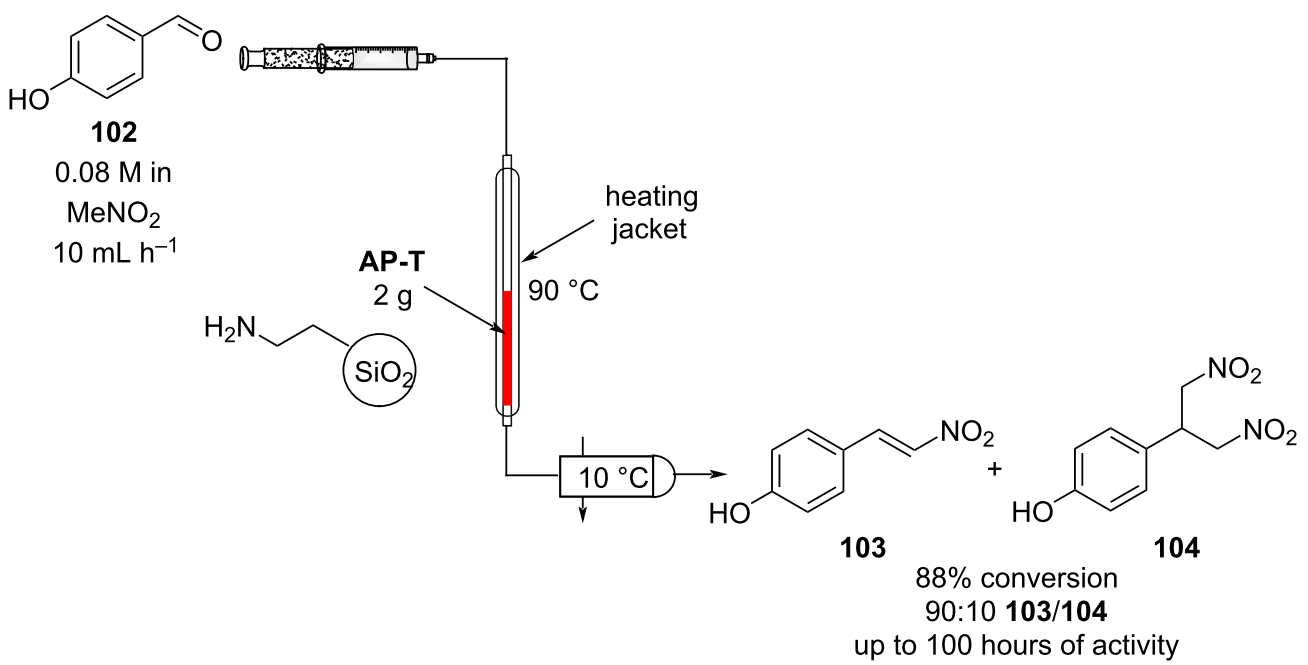

Scheme 22: Flow apparatus for the nitroaldol condensation of $p$-hydroxybenzaldehyde (102) to nitrostyrene 103 as described by Asefa et al.

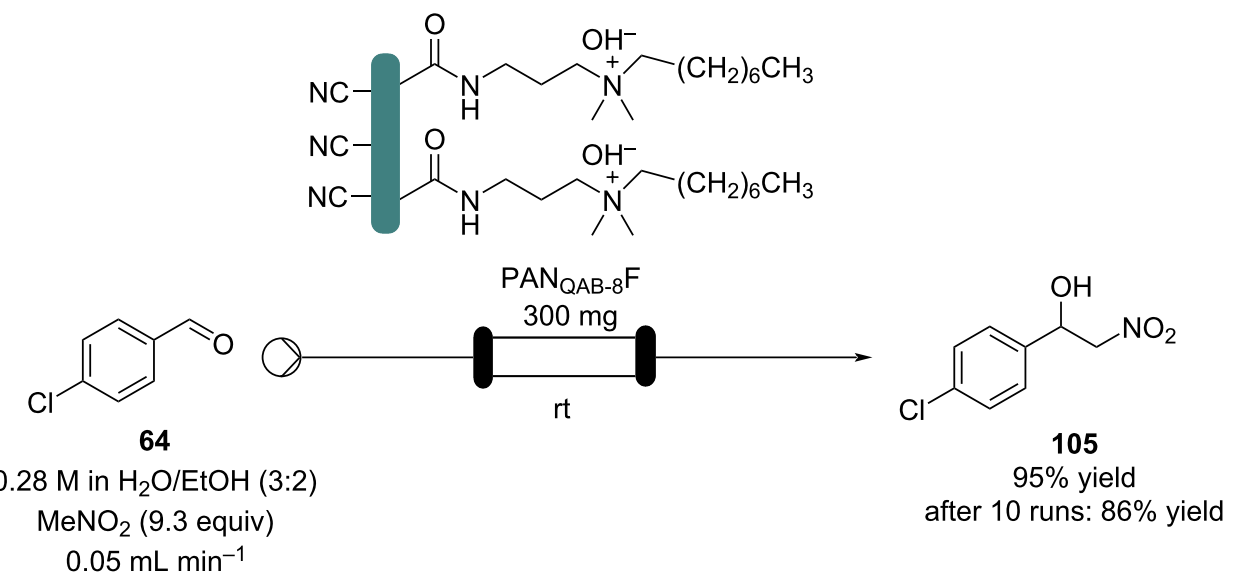


yield even after 160 hours of runtime) and reusability (after 10 runs $86 \%$ yield).

Packed-bed reactors have also been exploited to facilitate enantioselective nitroaldol processes. In 2013, Shibasaki and Kumagai made use of an entangling network of multiwalled carbon nanotubes (MWNT) to trap a Nd/Na bimetallic catalyst, which was shown to be an efficient combination for nitroaldol condensation (Scheme 24) [137].

In 2014, the authors modified their approach and packed the catalyst into a stainless steel column (with $19.5 \mu \mathrm{mol}$ in $\mathrm{Nd}$ ) to create a flow system. The catalyst column was cooled $\left(-40{ }^{\circ} \mathrm{C}\right)$ and a stream of aldehyde $\mathbf{1 0 6}$ was first passed through a precolumn containing MS $3 \AA$ and $\mathrm{NaHCO}_{3}$ to prevent poising from moisture and acids. Once mixed with the nitroethane, the mixture was directed into the catalyst. The catalyst was found to be highly efficient operating at high conversion (96-91\%) for 30 hours (TON $=204)$ with high enantio- and stereoselectivity (96:4 anti/syn, $91-92 \%$ ee). To confirm the ease of scaling the process, a larger column was prepared (with $0.294 \mathrm{mmol}$ in $\mathrm{Nd}$ ) and $12.4 \mathrm{~g}$ of $\mathbf{1 0 8}$ were obtained (93\% yield, 93:7 anti/syn, $88 \%$ ee, TON $=200$ ) after 28 hours [138].

Subsequently, the same group optimised the catalyst preparation using $\mathrm{NdCl}_{3} \cdot 6 \mathrm{H}_{2} \mathrm{O}$ and $t$ - $\mathrm{BuONa}$, equating to a 120 -fold less expensive system than the original employed salts. In this case, the flow system was operated for 24 hours with high conversion and selectivity (90\% yield, 20:1 anti/syn, $90 \%$ ee, TON = 145) [139]. The group also optimised the apparatus for the synthesis of other potential useful intermediates [140,141].
The introduction of $3 \mathrm{D}$ printing devices has aided flow chemistry, especially the prototyping of reactor designs (Scheme 25).

The possibility to quickly investigate several reactors of different volumes, shapes, and materials increases the chance for applicability and improves the understanding of the process itself [142-144]. Benaglia et al. exemplified 3D printing with a copper-catalysed Henry reaction [145]. Benzaldehyde (17) and the homogenous catalyst were mixed with nitroethane and the DIPEA base solution in a $1 \mathrm{~mL}$ polylactic acid (PLA) squarechannelled microreactor. The authors screened several other 3D printed reactors evaluating materials (PTFE, PLA or Nylon), volumes ( 1 or $10 \mathrm{~mL}$ ), and channel shape (circular, square, or rectangular). The process was then merged with a hydrogenation step using the $\mathrm{H}-\mathrm{Cube}{ }^{\circledR}$ apparatus $\left(\mathrm{Pd} / \mathrm{C}, 30^{\circ} \mathrm{C}, 15\right.$ bar) for the continuous preparation of 1,2-aminoalcohol 110 (90\% yield, anti/syn 7:3,78\% ee). At the end of the Henry reaction an in-line plug of silica gel removes the catalyst before the second reduction step to prevent issues of chelation and palladium deactivation.

Knoevenagel condensations are also of considerable interest to the F\&F industry (Scheme 26). The transformation is generally used to form $\alpha, \beta$-unsaturated acids or nitriles from condensation with aldehydes or ketones [146-151], however, there are also some examples where cyclic or tetra-substituted olefins are formed $[152,153]$.

The high processing volumes and short reaction times make the reactions quite suitable for continuous production via a flow system. Needless to say, the first example of Knoevenagel con-

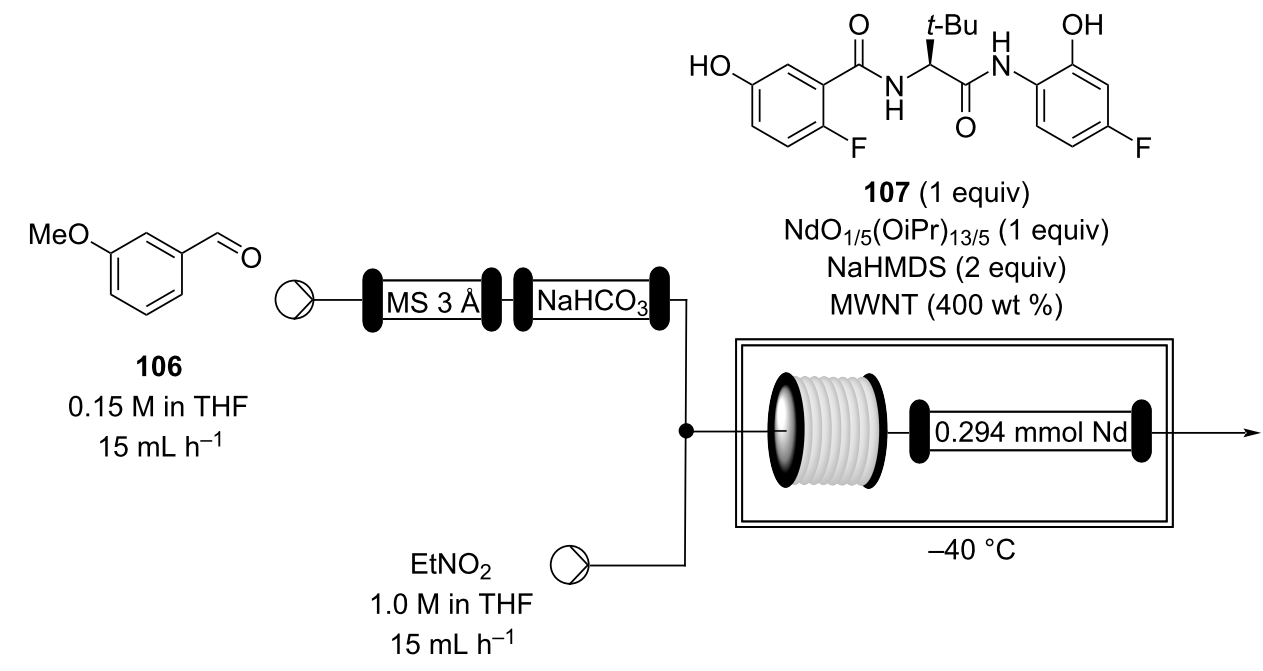

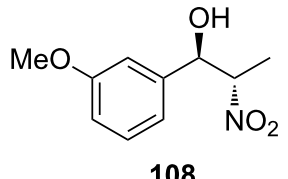

$93 \%$ isolated yield $\mathrm{dr}($ anti/syn $)=93: 7$

$88 \%$ ee

$28 \mathrm{~h}$ run

TON $=200$ 


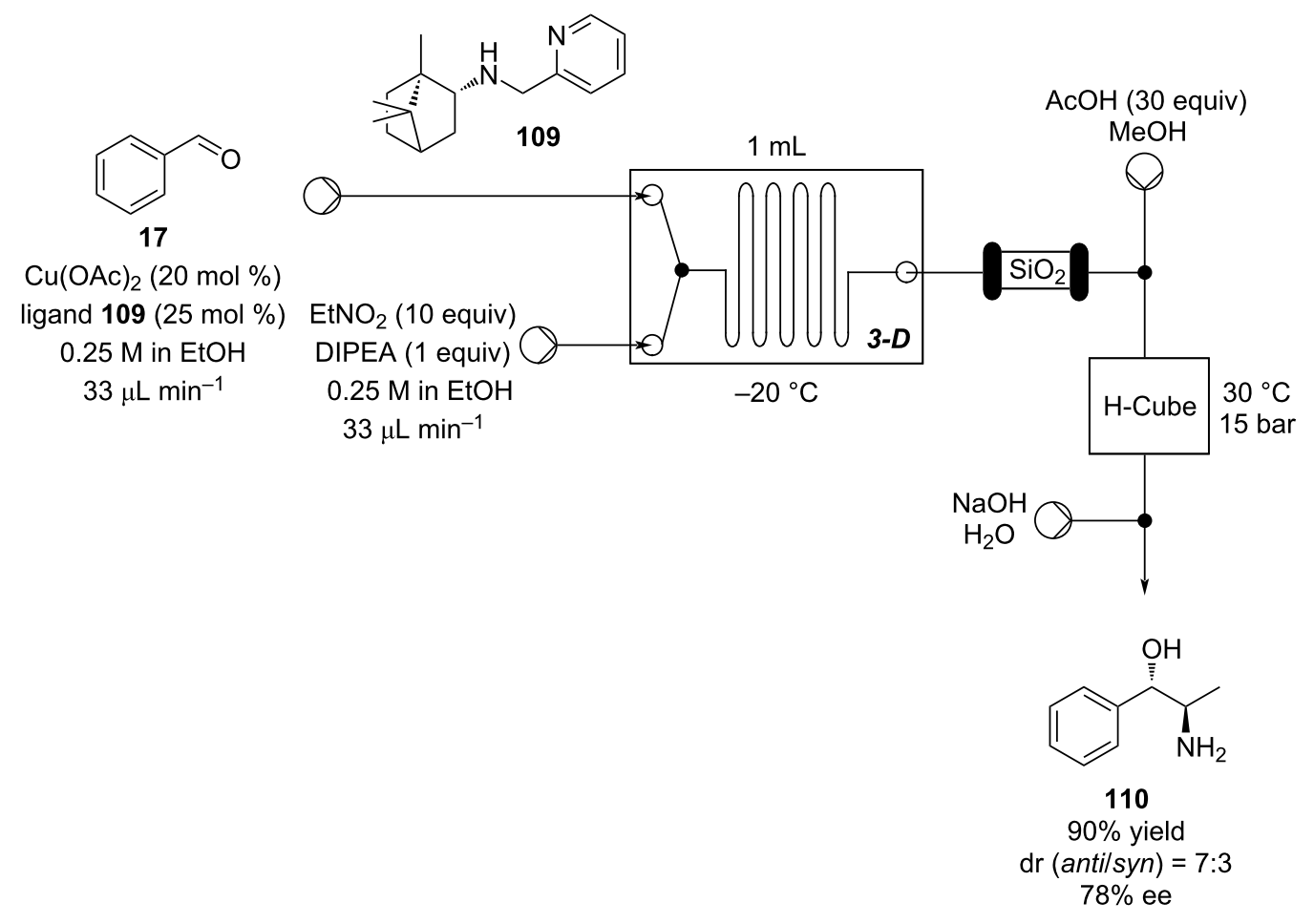

Scheme 25: Enatioselective synthesis of 1,2-aminoalcohol 110 via a copper-catalysed nitroaldol condensation.
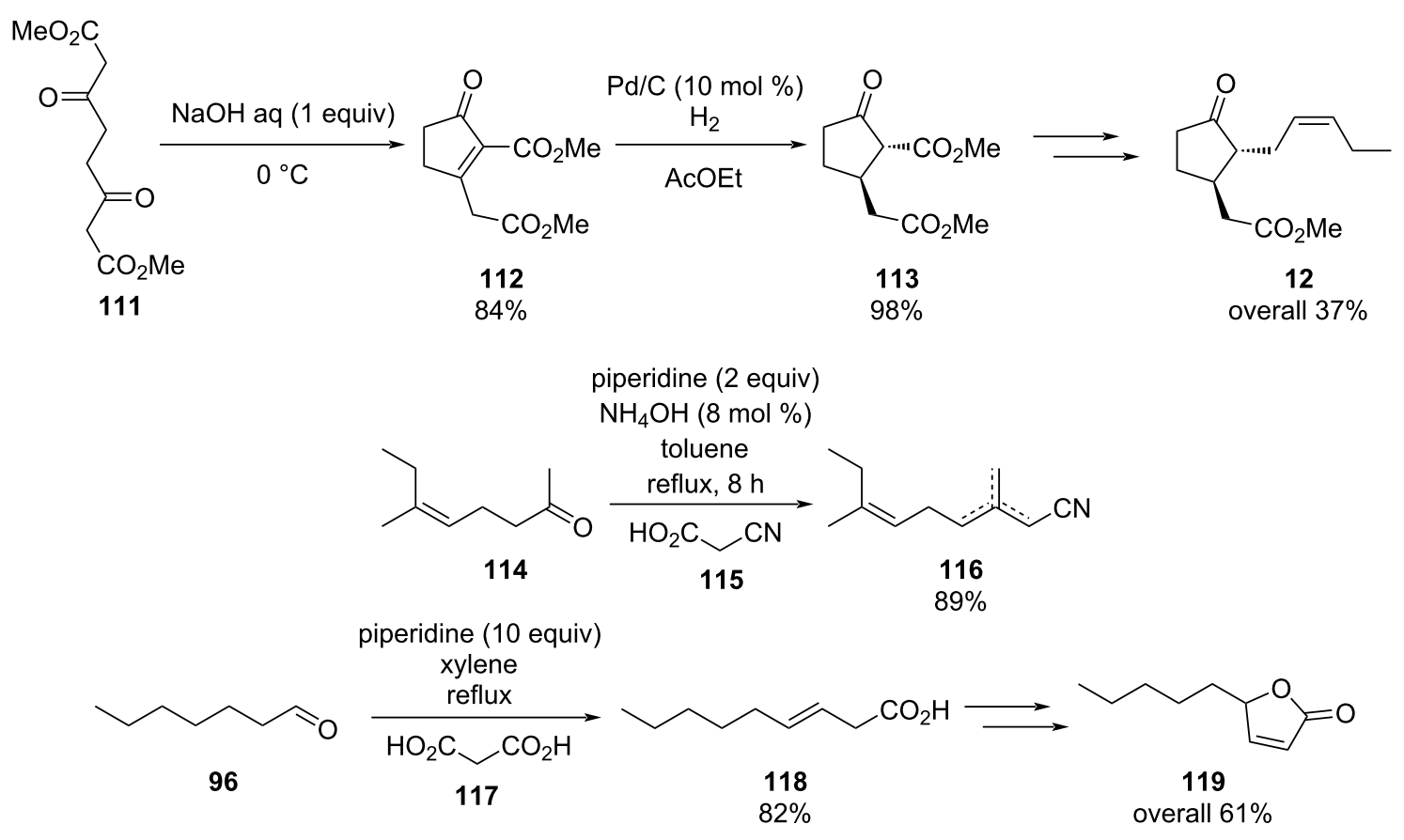

Scheme 26: Examples of Knoevenagel condensations applied for fragrance components.

densations in flow comes from Venturello et al. back in 1989 [154]. The authors exploited a glass column filled with 10 grams of aminopropyl-functionalised silica gel (AP-T). The reagents $(10 \mathrm{mmol})$ were eluted through the column in the same way as gravimetric chromatography is performed (Scheme 27). 


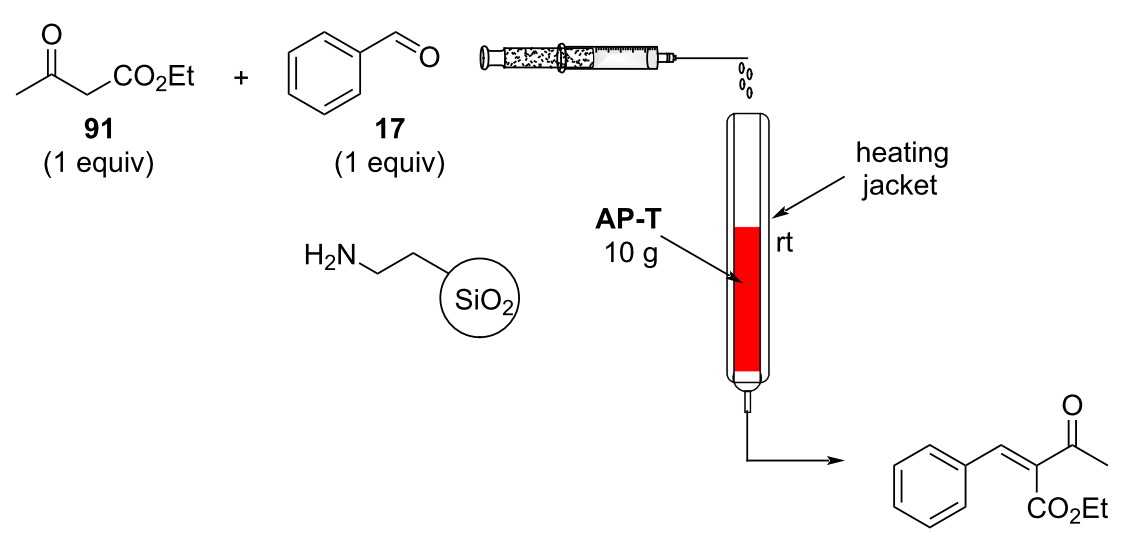

120

$59 \%$ yields

$81 \%$ conversion

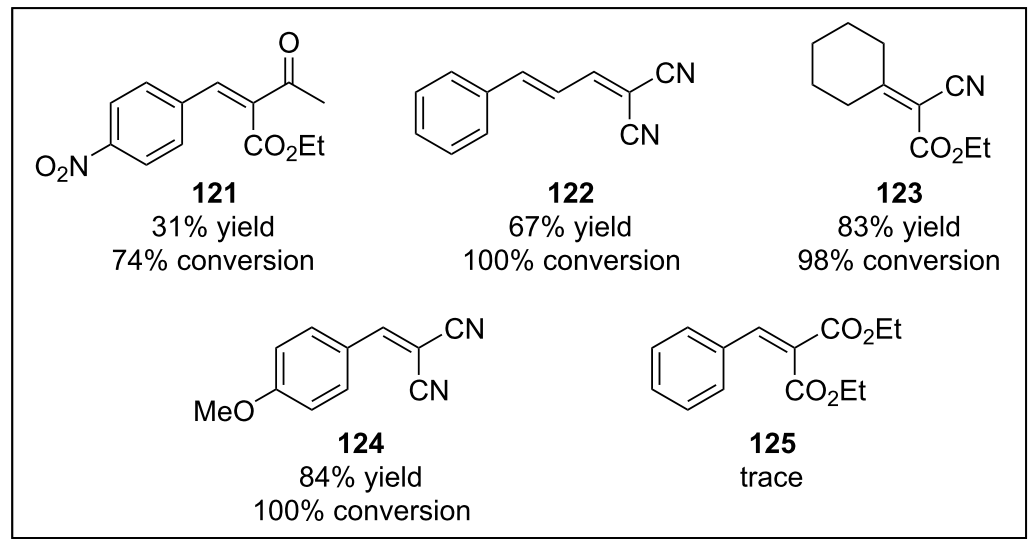

Scheme 27: Flow apparatus for Knoevenagel condensation described in 1989 by Venturello et al.

In this way, the authors claimed the product was completely collected after $250 \mathrm{~mL}$ of toluene elution. The silianol groups on the AP-T silica are suggested to play a key role in the water elimination step. Furthermore, the primary amine function on the silica shows better outcomes compared to a tertiary amine. Different products were prepared in moderate to excellent yields, however, with the less acidic diethyl malonate the catalyst proved to be less active.

In 2013, the Knoevenagel reaction between benzaldehyde (17) and malononitrile was performed using a similar flow apparatus, however, using a polystyrene-supported DABCO as catalyst. Wang's group confirmed the stability of the latter, which provides excellent conversions (95-90\%) over 10 hours of continuous runtime [155]. As such a simple chromatographic column can be exploited as flow reactor to perform initial feasibility tests.

As with all condensation reactions, the main problem when performed in flow is the elimination of water. Yeung et al. provided a solution using a coated multichannel microreactor with an integrated zeolite ZSM-5 membrane (Scheme 28) [156,157]. The heterogeneous catalyst used was a Cs-exchanged faujasite $\mathrm{NaX}$. The membrane maximised the outcomes in comparison to tested fixed-bed and microreactors. The ZSM-5 membrane allowed fast water exclusion, however, long residence times (5 hours) were required. The authors claimed a more active catalyst would reduce the reaction times. As a result, over the years, the same group has provided improved alternative microreactors and catalysts; for example, a capillary microreactor coated with Cs-exchanged faujasite $\mathrm{NaX}$ was reported [158], along with a ZIF-8/NaA composite membrane microreactor [159]. These systems embodied more active and stable catalysts which were able to consequently operate for over 50 hours with delivery of excellent product yields $(>95 \%)$. Also these systems, instead of using membranes for the water removal instead employed either DMSO or ionic liquids as additives for the water removal.

The same condensation (of $\mathbf{1 7}$ to $\mathbf{1 2 7}$ ) was also performed by Clark et al. using a silica-coated microreactor with an AP-T catalyst deposited on the reactor's walls [160]. The solvent-free 


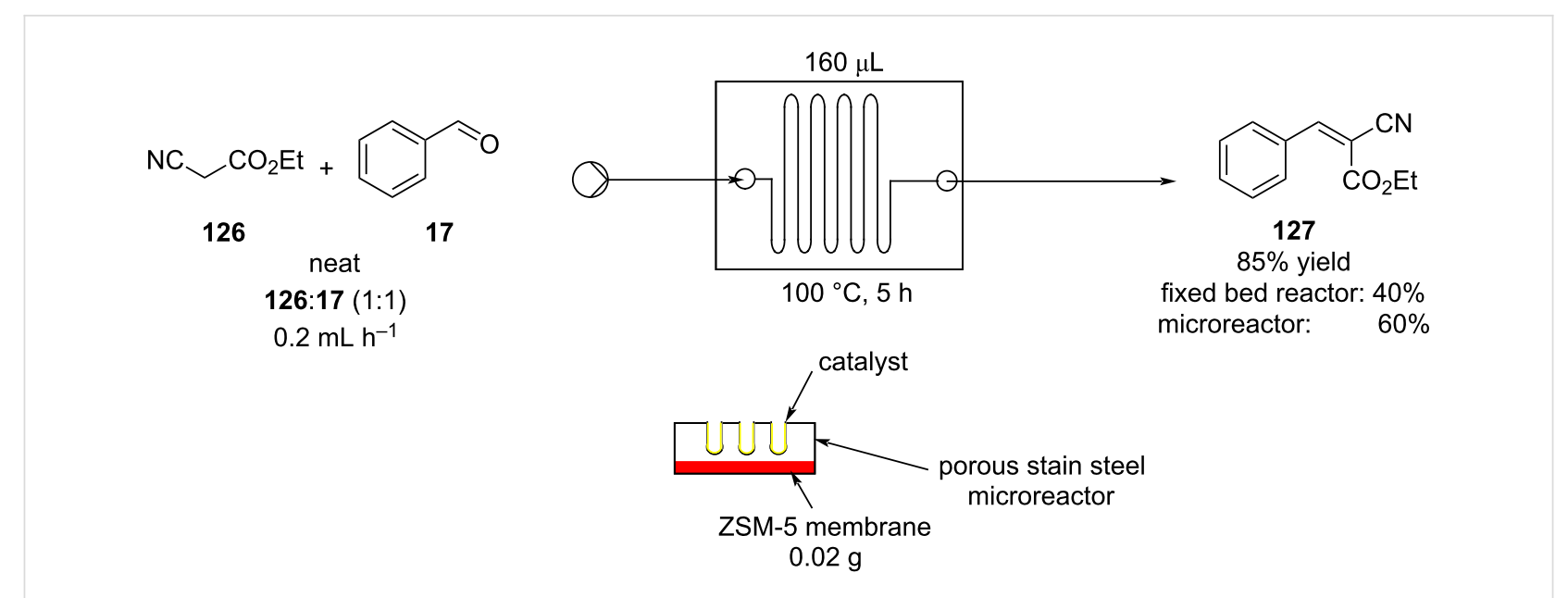

Scheme 28: Knoevenagel reaction using a coated multichannel membrane microreactor.

approach brought about the product $\mathbf{1 2 7}$ in roughly $80 \%$ yield, however, effects of poisoning and catalyst leaching reduces the conversion by $20 \%$ over 8 hours of runtime. The poising was attributed to the presence of stationary areas caused by nonuniform flow in the reactor. Generally, this issue could be addressed by changing the configurational shape of the apparatus so to increase the mixing and flow uniformity. Nonetheless, new technique could also be applied, such as electroosmotic flow (EOF). The later technique has been widely used in flow for its incredible advantages compared to common pressure-driven systems [161]. Watts and Nikbin employed EOF in a piperazine-functionalised silica-catalysed Knoevenagel rreaction for the preparation of $\mathbf{1 2 7}$. The authors managed to reduce the reaction times ( 2 hours vs 5 minutes) and yielded the product in $82 \%$ conversion, confirming the applicability of EOF technology in heterogeneous catalysed-flow systems [162].

Many of the products, such as $\mathbf{1 2 7}$ and its derivatives, are considered potential intermediates in the pharmaceutical industry [163,164], many other applications in flow have been described improving the catalyst stability. In 2016, Tao's group developed a bioinspired metal-oxide-immobilized monolith, which was optimised for several reactions (reduction, esterification, and Knoevenagel) [165]. The authors employed a hierarchically porous silica (HPS) coated with a pyrogallol-based polyphenol film (PG). This type of supported system allowed incorporation of a greater amount of metal oxide compared to the standard impregnation. For the Knoevenagel reaction, $\mathrm{Al}_{2} \mathrm{O}_{3}$ was immobilised on the PG-HPS. The reagents were streamed into the reactor at $0.05 \mathrm{~mL} \mathrm{~min}{ }^{-1}$ (roughly 30 minutes residence time) at $80{ }^{\circ} \mathrm{C}$, with compound $\mathbf{1 2 7}$ being obtained in $89 \%$ conversion. The apparatus could be operated for over 24 hours, however, leaching of $\mathrm{Al}_{2} \mathrm{O}_{3}$ reduces the conversion to $76 \%[166]$.
The preparation of a heterogeneous catalyst often has higher associated costs, therefore once applied the species must be efficient and stable to generate over time good economic margins. Consequently, developing supports for stable catalysts in flow is becoming a major research task. To manage costs and increase the greenness of the process, many new supports have been developed. An interesting example are aerogels which present low densities, large surface areas, and interconnected porous structures which makes them highly suitable for low pressure drop column based catalysis [167]. In 2014, Isogai et al. employed nanofibrillar chitin aerogel as a potential sustainable heterogeneous catalyst for Knoevenagel condensations. The catalyst, presenting the amine functional groups on its surface, was loaded in a syringe along with the mixture of reagents (benzaldehyde and ethyl acetonitrile) and pumped at $0.01 \mathrm{~mL}$ $\mathrm{min}^{-1}$ at room temperature. The authors managed to obtain compound 127 in good to excellent yields (85-99\%), and the catalyst could be reused over 5 times without signs of detriment [168]. In 2017, Ma et al. developed a sustainable aminefunctionalised catalyst using sugarcane bagasse (SCB) as a support (Scheme 29). The as-prepared catalyst was found to be highly active for effecting Knoevenagel condensation at room temperature [169].

Polyacrylonitrile fibres (PNAF) were also successfully employed as alternative supports by the groups of Ma and Zhang in 2019. The supports were functionalised with different bases, such as 1,8-bis(dimethylamino)naphthalene, and prolinamide, and provided excellent mechanical properties and stability to the catalysts which could be reused successfully several times [170,171].

Knoevenagel reactions of aliphatic aldehydes are much more challenging, often the freshly formed double bond 


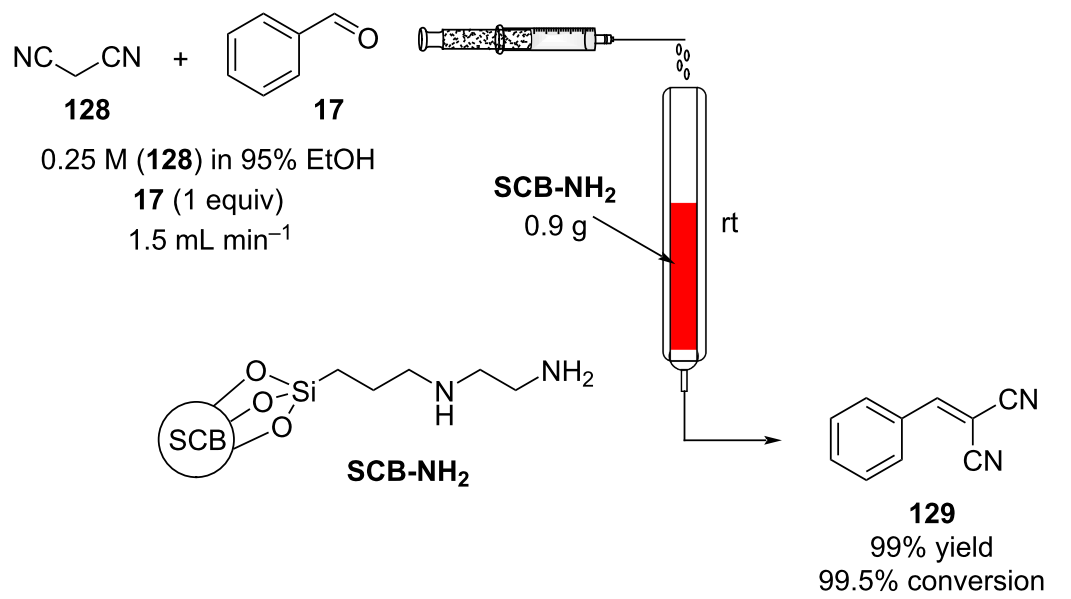

Scheme 29: Continuous-flow apparatus for Knoevenagel condensation employing sugar cane bagasse as support developed by Ma et al.

can further react and form Michael adducts with the more reactive starting materials. Kobayashi et al. developed an efficient flow apparatus able to overcome such issues. A primary amine-functionalised silica (Chromatorex $\mathrm{NH}$ ) blended with molecular sieves $4 \AA$ (MS 4 Å) were employed to increase the conversion toward the condensed products. The compounds 131-135 (Scheme 30) were gained in high to excellent yields compared to the corresponding batch method. In particular, species $\mathbf{1 3 1}$, an intermediate in the synthesis of pregabalin (138), was obtained in $>95 \%$ yield, which could be maintained for over 50 hours. Using a longer column $(10 \times 200 \mathrm{~mm}$ vs $10 \times 100 \mathrm{~mm})$, the apparatus operated over 100 hours with no signs of deterioration $(90-100 \%)$.

The flow system was coupled with other reactor units to develop a continuous synthesis of compound 137, an intermediate for ( \pm )-pregabalin (138; Scheme 31). From compound 130,

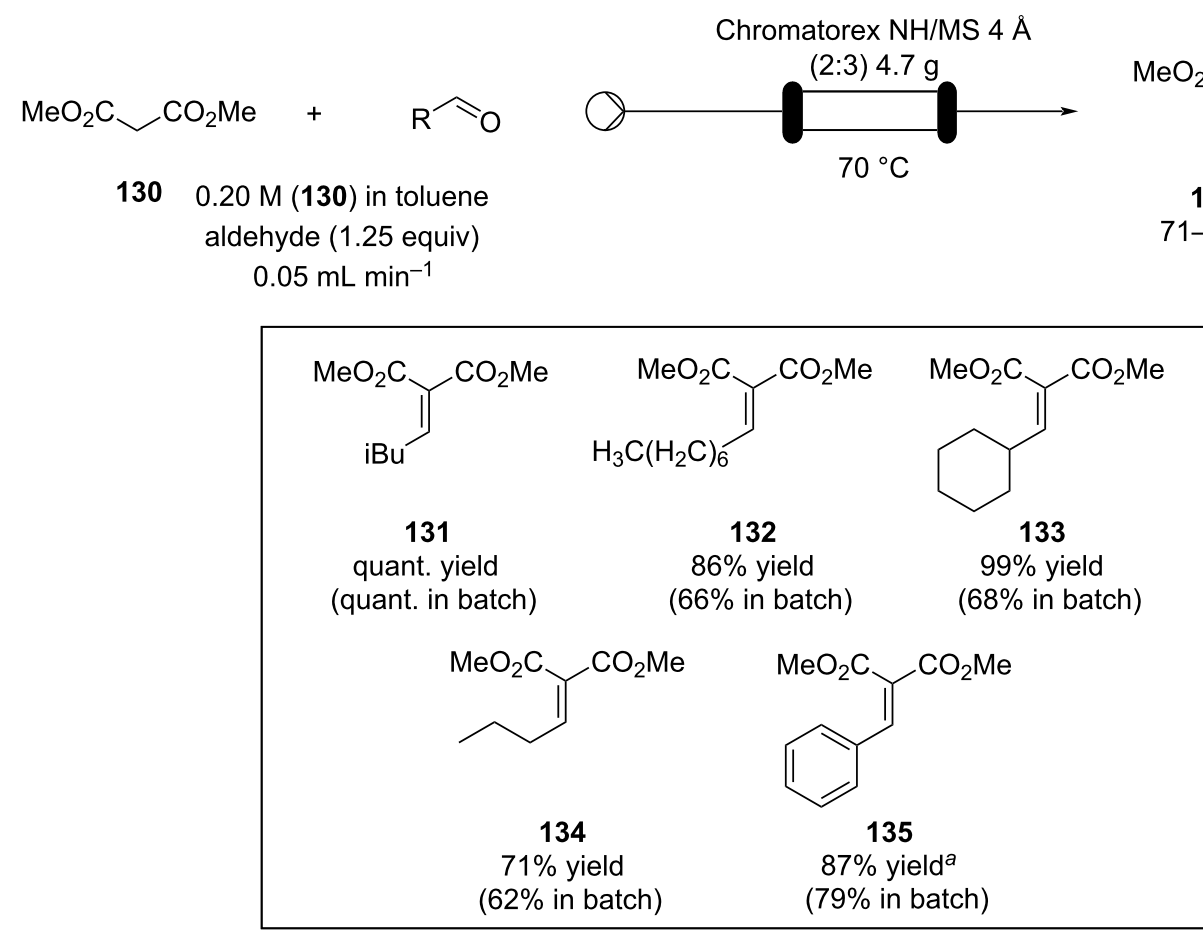

Scheme 30: Knoevenagel reaction for the synthesis of 131-135 in flow using an amine-functionalised silica gel. ${ }^{\mathrm{a}} \mathrm{A} 10 \times 200 \mathrm{~mm}$ column was used instead of a $10 \times 100 \mathrm{~mm}$ column. 
a Michael reaction with nitromethane catalysed by basic Amberlite ${ }^{\circledR}$ IRA900 (hydroxide form), installed the nitro group which was hydrogenated using a poly-silane-supported bone charcoal palladium catalyst previously developed within the same group [172].

The cyclisation occurs as soon as the amine group is formed (137). The authors revealed a column filled with MS $5 \AA$ and silica had to be placed between the second and third step as low conversions were detected over the time (59-78\%). The pre-column acts as an in-line purification for unreacted reagents or any byproducts formed during the first parts of the process. The following flow setup allowed $\mathbf{1 3 7}$ to be formed in $76-99 \%$ yields over 45 hours of runtime (space time yield $\left.52.2 \mathrm{~g} \mathrm{~L}^{-1} \mathrm{~d}^{-1}\right)$.

To fully satisfy the 12 principles of greenness, the Knoevenagel reactions can also be exploited in a continuous solventfree process using screw reactors [173-175]. In this particular example, a twin-screw extruder (TSE) was employed. The reactants were added as solids in the apparatus. The rotating screws push the solid forwards, mixing the solid reactants together. The extruder has several heating channels which can be individually set at different temperatures. Once the mixture of vanillin (2), barbituric acid (139), and sodium carbonate were heated at $160{ }^{\circ} \mathrm{C}$ for 2 minutes, the product 140 was obtained quantitatively. The TSE apparatus was able to manufacture the material at $0.520 \mathrm{~kg} \mathrm{~h}^{-1}$.
Solid-liquid systems can also be exploited simply by adding a syringe pump inlet to the extruder. Malononitrile and ethyl cyanoacetate were used as examples, and the products 141 and 142 were produced in quantitative yield with extremely high throughputs (Scheme 32).

The authors also described the telescoping of a Knoevenagel-Michael addition process for the synthesis of $\mathbf{1 4 3}$. The optimised method enables isolation of the desired material quantitatively at $154 \mathrm{~g} \mathrm{~h}^{-1}$. A one-pot like process, where all the solids were fed from the same feeder, was also developed proving the potentiality of this technique for immediate industrial applications.

The Darzens reaction can also be considered as a condensation type reaction. Darzens products (epoxides, aziridines) are valuable building blocks, especially for the pharmaceutical and agrochemical industries [176-178]. Although a great number of publications are present in the literature, few continuous approaches have been described thus far. In 2001, Lewis et al. published a conference paper describing a continuous phasetransfer-Darzens condensation of a ketone for the preparation of pharmaceuticals (Scheme 33) [179].

A spinning disc reactor (SDR), which is depicted in Scheme 33, was employed. The solution of starting materials entered from the centre of the disc and drained out from a vessel which is placed below the disc assembly. The spinning applies a very

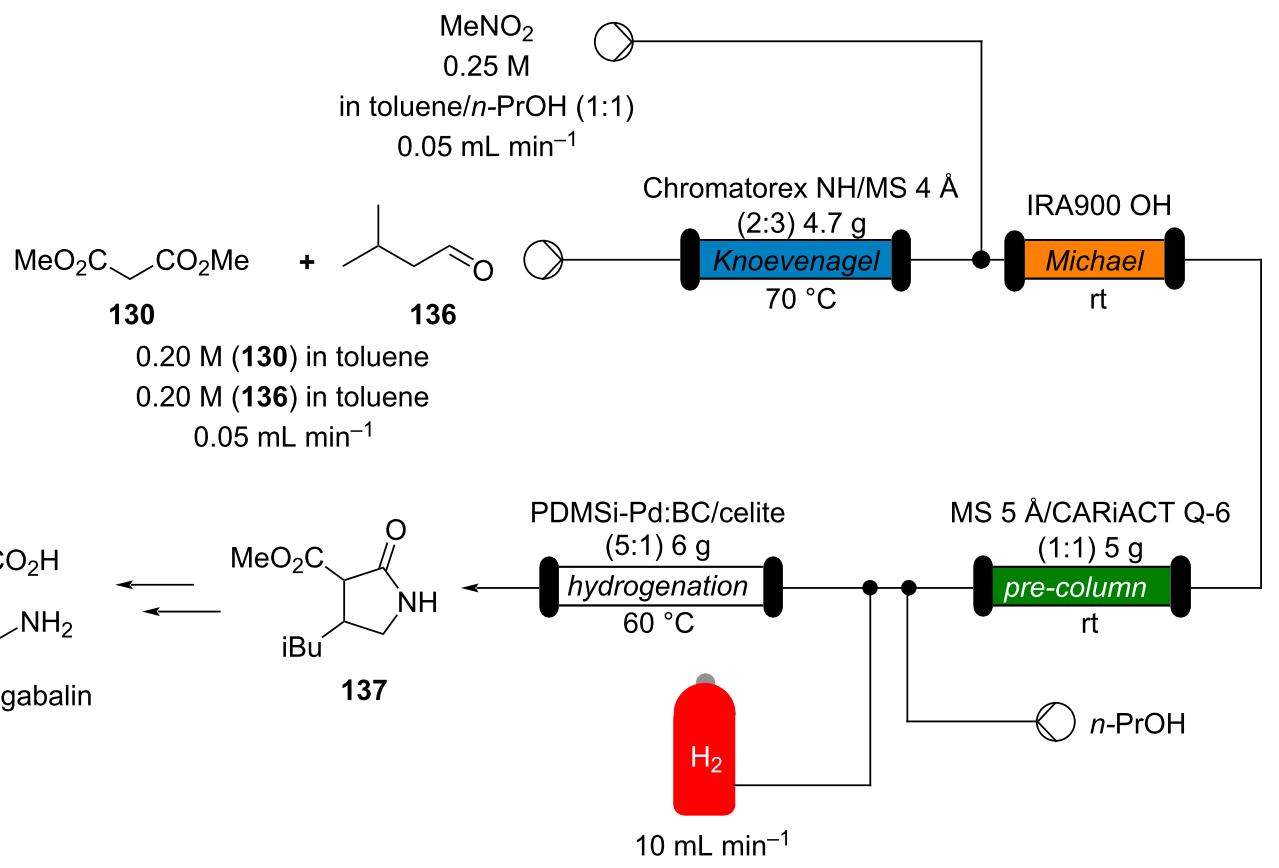

Scheme 31: Continuous-flow synthesis of compound 137, a key intermediate for the synthesis of pregabalin (138). PDMSi-Pd/BC: polysilane/bone charcoal-supported Pd catalyst. CARIACT Q-6: silica support. 
<smiles>COc1cc(C=O)ccc1OCCOC(=O)O</smiles>

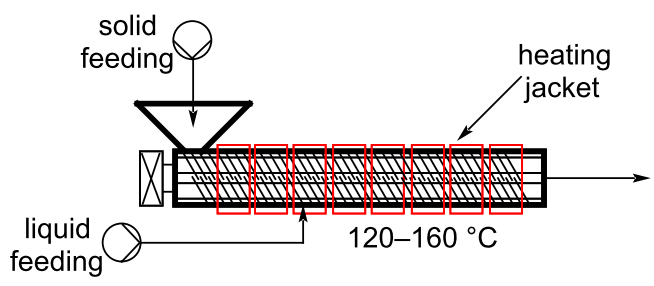<smiles>COc1cc(C=C2C(=O)NC(=O)NC2=O)ccc1O</smiles>

140

$0.520 \mathrm{~kg} \mathrm{~h}^{-1}$

$\left(258385 \mathrm{~kg} \mathrm{~m}^{-3} \mathrm{~d}^{-1}\right)$

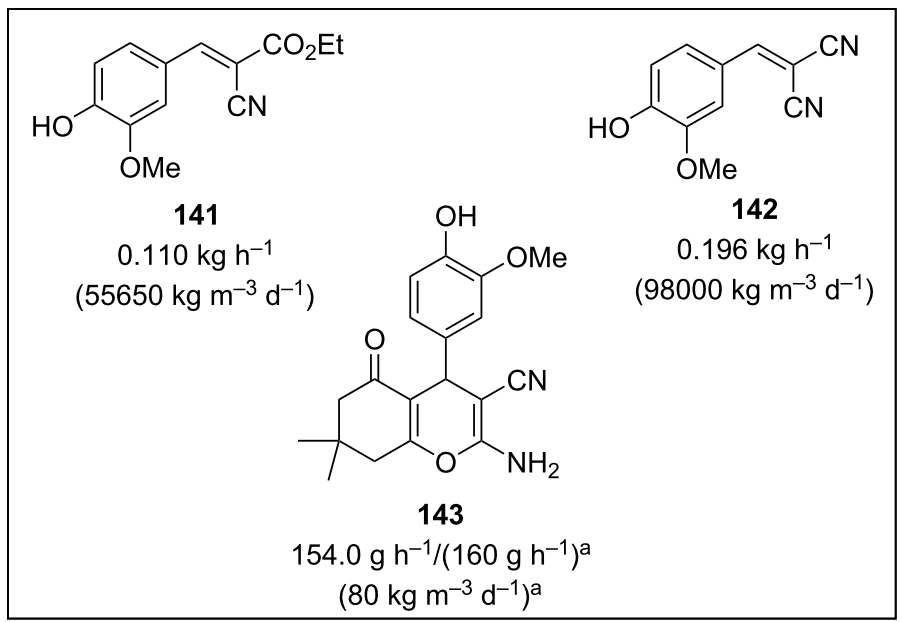

Scheme 32: Continuous solvent-free apparatus applied for the synthesis of compounds 140-143 using a TSE. Throughput rate and space time yields (in brackets) are reported. aThe data are from the multicomponent reaction process instead of the Knoevenagel-Michael addition telescoped one.

$$
\mathrm{R}^{1} \stackrel{\mathrm{O}}{\mathrm{R}^{2}}+{ }_{144}^{\mathrm{Cl}} \underset{\mathrm{CN}}{\sim}
$$

$\mathrm{KOH}$ aq (16 equiv)

unknown phase-transfer catalyst

$1.5 \mathrm{~mL} \mathrm{~s}^{-1}$
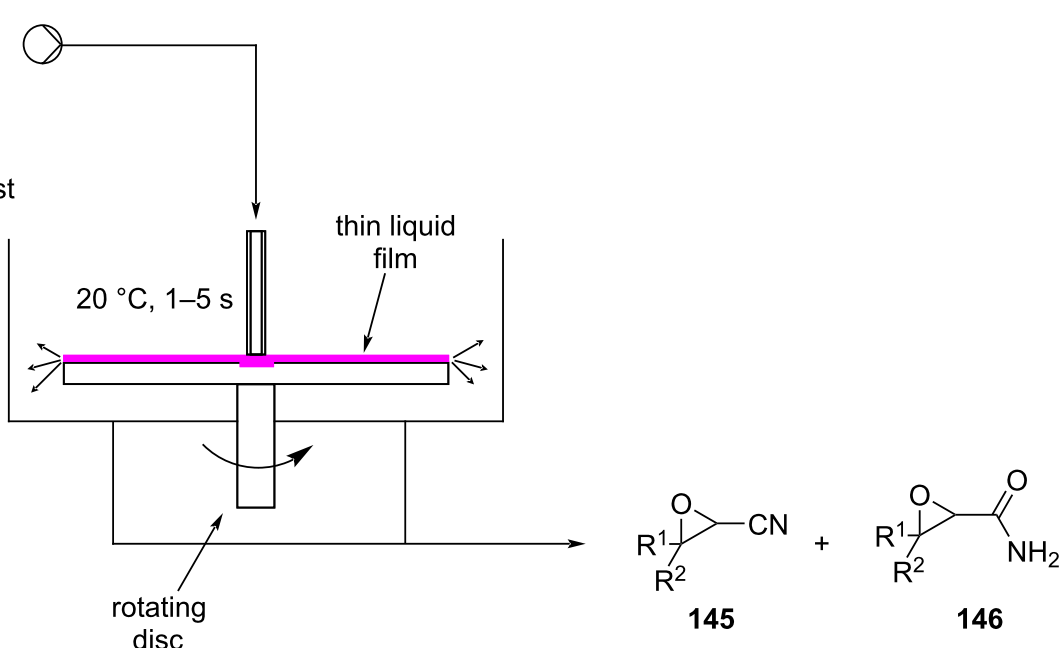

Scheme 33: Lewis et al. developed a spinning disc reactor for Darzens condensation of $\mathbf{1 4 4}$ and a ketone to furnish the $\alpha, \beta$-epoxynitrile $\mathbf{1 4 5}$. 
high shear stress on the thin film (thickness ranges 50-600 $\mu \mathrm{m}$ ) which leads to very high mass and heat transfer. The residence time is around 1-5 seconds, and the disc spinning was maintained at $4500 \mathrm{rpm}$. The system allowed for operation under milder reaction conditions $\left(20^{\circ} \mathrm{C}\right)$ compared to the original batch process $\left(0{ }^{\circ} \mathrm{C}\right)$, and to give improved control over the formation of the byproduct $146(0.1 \%$ vs $1.4 \%)$. The authors highlighted the linear relationship between the disc spinning rate and the product formation, suggesting better mixing was a key factor in the reaction's outcomes. To improve the efficiency of the mixing, a textured disc with a counterclockwise spiral shape carved on its surface was employed. The desired product 145 was obtained in $88 \%$ conversion with a calculated throughput of 8 tonnes per year.

\section{Conjugate addition}

Conjugate addition reactions are commonly utilized in synthetic fragrance chemistry to generate new $\mathrm{C}-\mathrm{C}$ and $\mathrm{C}$-heteroatom bonds. The Michael addition, discovered in 1887 by Arthur Michael [180], has clearly been a milestone in the field of organic chemistry. Being versatile in the substrates and operationally simple to perform, Michael additions immediately found widespread industrial applications. Several examples of fragrance preparation using conjugate additions and more specifically Michael additions are described in the literature. Dihydrocoumarin (152) [181,182], methyl dihydrojasmonate (33) [183], resorcinol esters such as veramoss (6) [184], and many other well-known fragrances and cosmetic additives are industrially prepared via such related routes (Scheme 34).

Flow chemistry has also been enacted to assist in several conjugate additions, reducing associated safety risks and improving selectivity. The continuous-flow synthesis of 4-(2-hydroxyethyl)thiomorpholine 1,1-dioxide (156), a key intermediate for the synthesis of the HIV maturation inhibitor (MI) BMS955176 illustrates the benefits with respect to reduced handling of toxic/reactive intermediates and easy scaling to kilogram levels (Scheme 35) [185].

When nucleophiles containing an active methylene group are involved, the addition reaction can often be performed using catalytic amounts of base. Over time a broad range of different catalysts have been evaluated resulting in varying conversions and associated levels of regio- and stereoselectivity for the addition process [186-189]. Several solid inorganic bases have been considered as heterogeneous catalysts for Michael addition [190], for example, caesium fluoride on alumina has been recently exploited in the continuous-flow synthesis of glutamic acid derivatives (Scheme 36). The flow setup devised was used
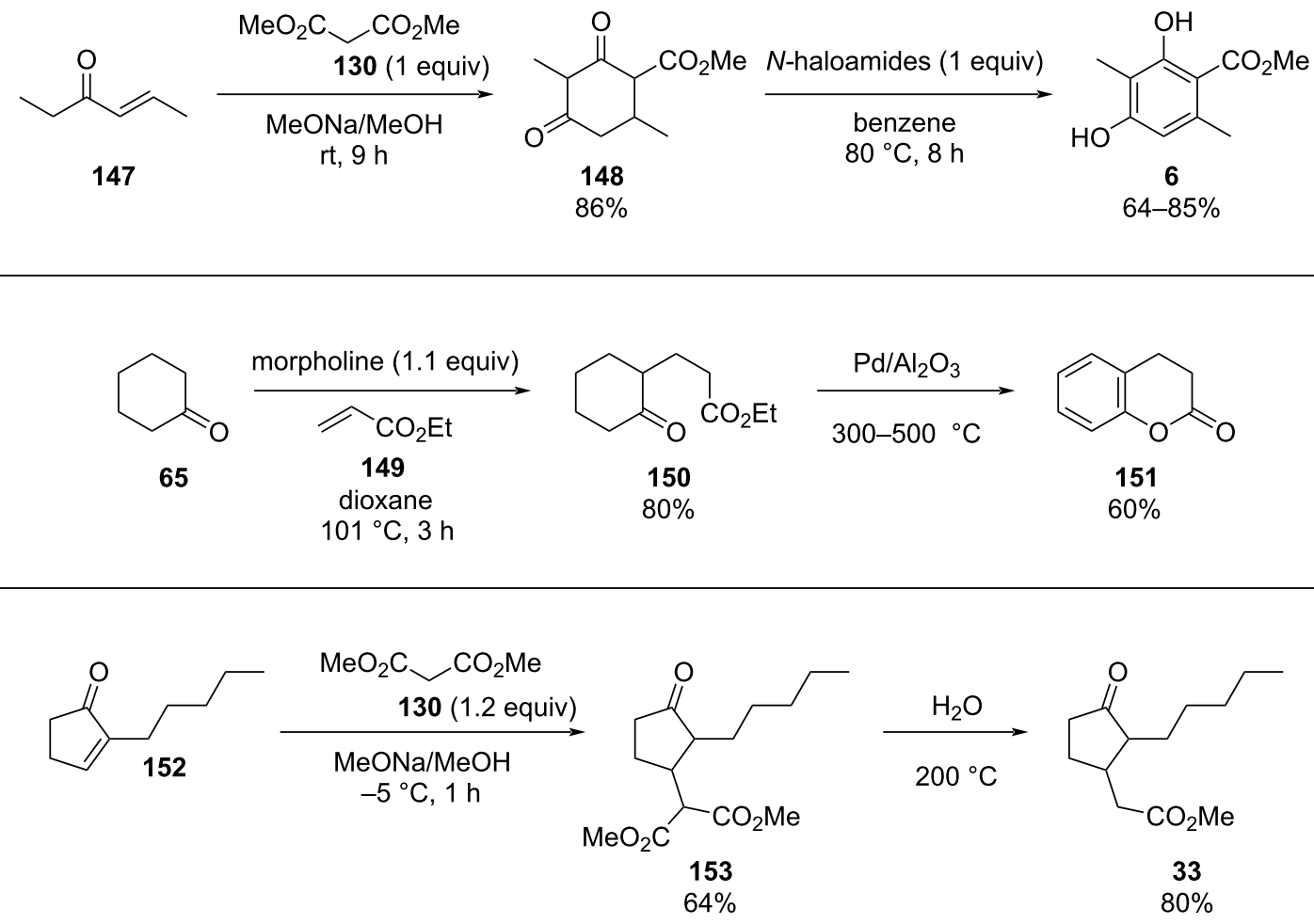


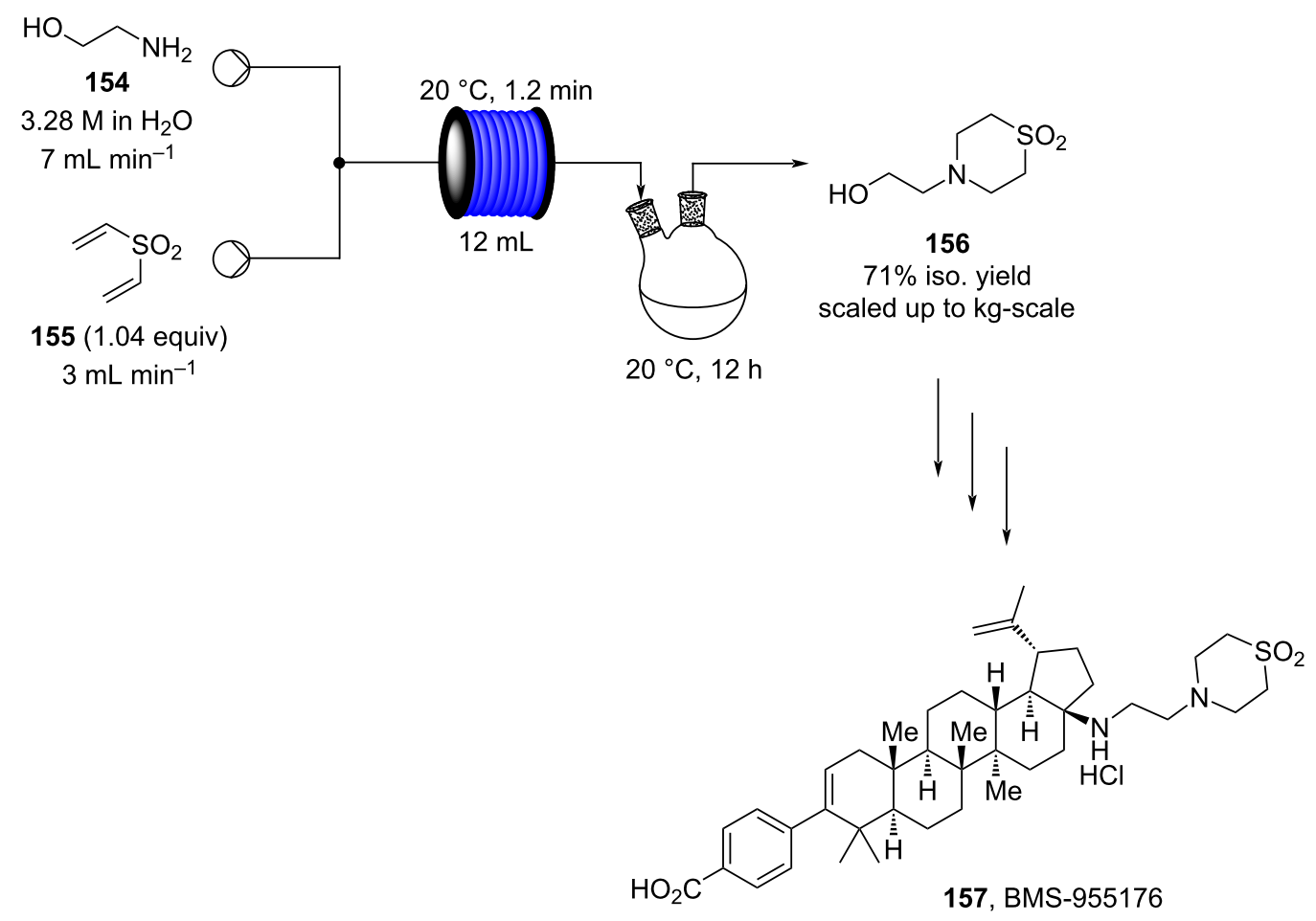

Scheme 35: Continuous-flow synthesis of 4-(2-hydroxyethyl)thiomorpholine 1,1-dioxide (156) via double conjugate addition on route to BMS-955176 (157).

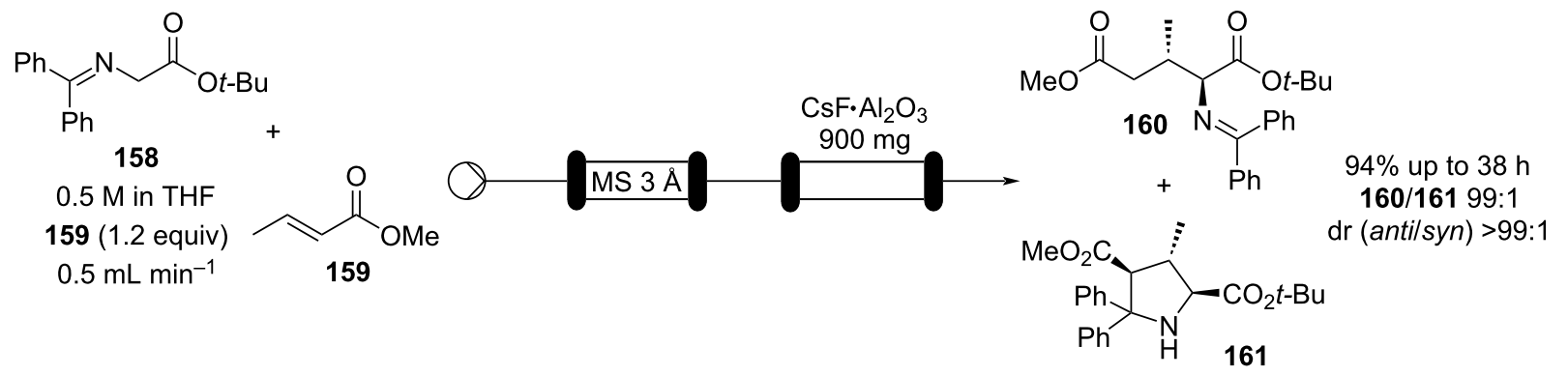

Scheme 36: Continuous-flow system for Michael addition using CsF on alumina as the catalyst.

to reduce the residence time (increase throughput) and suppress the competitive $[3+2]$ cycloaddition generating the pyrrolidine compound 161. It was demonstrated that the flow system could be continuously run for 38 hours without any sign of decline in conversion or selectivity [191].

Similarly, calcium chloride has been used to promote asymmetric addition of malonates into unsaturated nitro compounds using an immobilised chiral bisoxazoline ligand in a flow process (Scheme 37) [192]. The reactor setup proved highly efficient with activated aromatic and heteroaromatic nitrostyrenes 163-170, however, it showed reactivity limitations when aliphatic $\alpha, \beta$-unsaturated nitro compounds were employed (171).

This procedure proved highly advantageous for adaption to the multistep continuous-flow synthesis of $(R)$-rolipram (Scheme 38) [193].

Starting from aldehyde 172, a Henry condensation was enacted to prepare the corresponding nitrostyrene. After performing the Michael addition, as described above, hydrogenation using a developed polysilane-supported palladium/carbon (Pd/DMPSi-C) occurred. The flow stream was then degassed in a syringe filled 


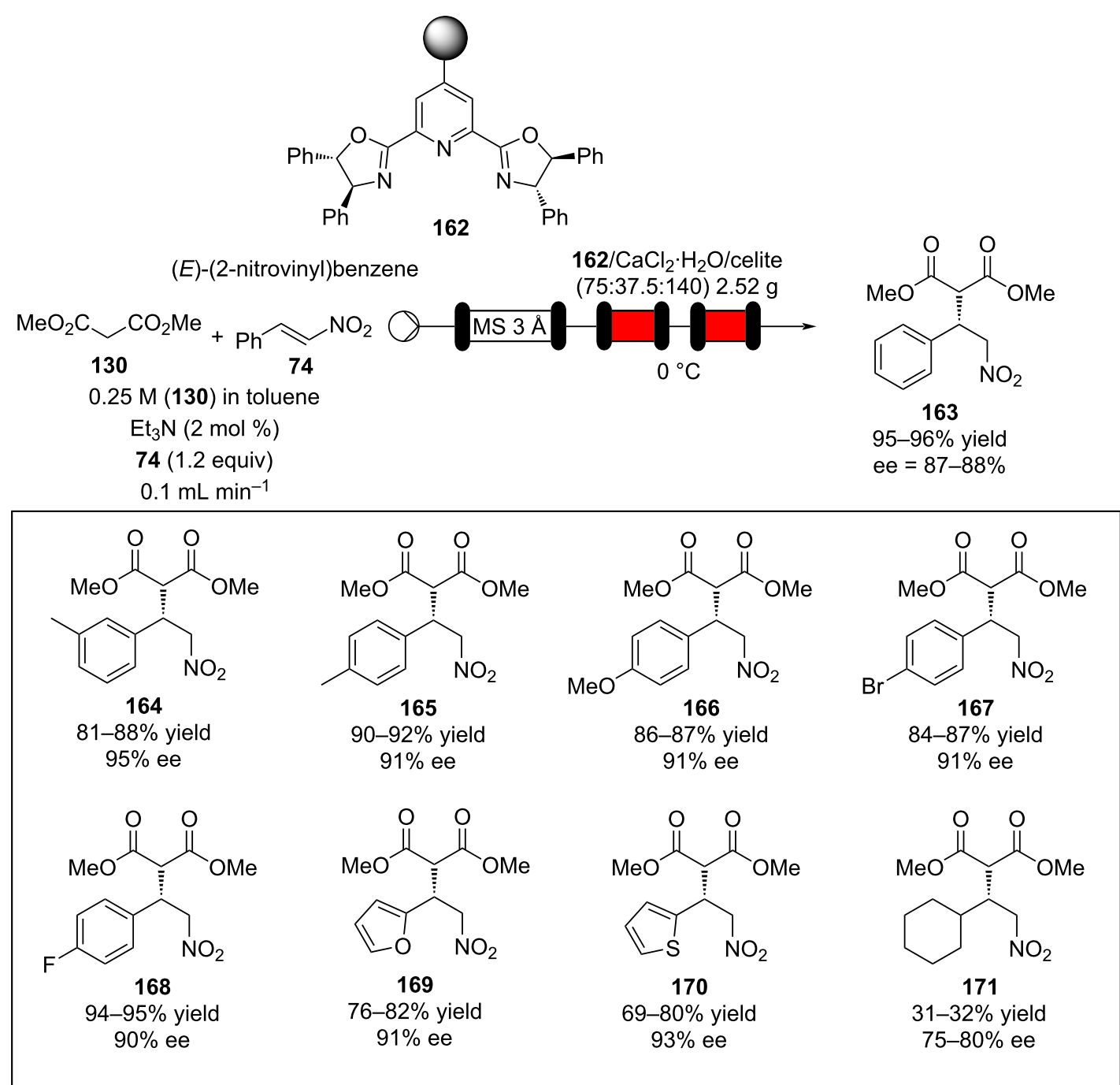

Scheme 37: Calcium chloride-catalysed asymmetric Michael addition using an immobilised chiral ligand.

with a sulfonic acid resin, which contributes to the ring formation. Decarboxylation was accomplished using a silica-supported carboxylic acid (Si-COOH) column at $120^{\circ} \mathrm{C}$ streamed from top to bottom. The desired material was gained in 50\% yield, and roughly 1 gram of enantiopure ( $>99 \%$ ee) $\mathbf{1 7 3}$ was isolated after 24 hours of collection.

The use of several common ion exchange resins have been tested as catalysts for Michael addition between 2-methylindole and several $\alpha, \beta$-unsaturated ketones and nitro compounds (Scheme 39).

Dried Amberlyst ${ }^{\circledR}$ A26 (hydroxide form) was shown to be the most efficient species resulting in good to excellent yields. The synthesis of $\mathbf{1 7 6}$ in flow was demonstrated, the simple packed column system allowed easy isolation of the product and the catalyst activity was maintained over 10 hours [194]. A similar approach using Amberlyst ${ }^{\circledR}$ IRA-900 (OH form) was employed for the Michael addition in the multistep flow synthesis to a key intermediate of pregabalin (173) by Kobayashi et al. (Scheme 38) [172].

Organocatalysis has been widely employed for Michael addition reactions and several metal-free enantioselective versions have been reported over the years [101,195,196]. However, most systems suffer from catalyst deactivation which necessitates the use of high catalyst loading but still reduces productivity over time. Heterogeneous catalysis paired with flow chemistry has proven a fertile area of research [197]. Systems using silica gel as a supporting matrix have been widely employed. For instance, Paixão et al. developed a silica-supported proline-based peptide 181, whose preparation requires only three steps exploiting a Ugi multicomponent reaction (Scheme 40) [198,199]. 
<smiles>COc1ccc(C=O)cc1OC1CCCC1</smiles>

$0.2 \mathrm{M}$ in toluene $\mathrm{MeNO}_{2}$ (1.2 equiv)

$50 \mu \mathrm{L} \min ^{-1}$

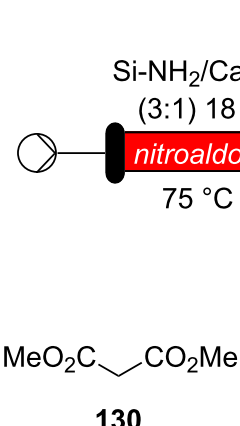

130

$0.22 \mathrm{M}$ in toluene

$\mathrm{Et}_{3} \mathrm{~N}(7.2 \mathrm{~mol} \%)$

$50 \mu \mathrm{L} \mathrm{min}^{-1}$

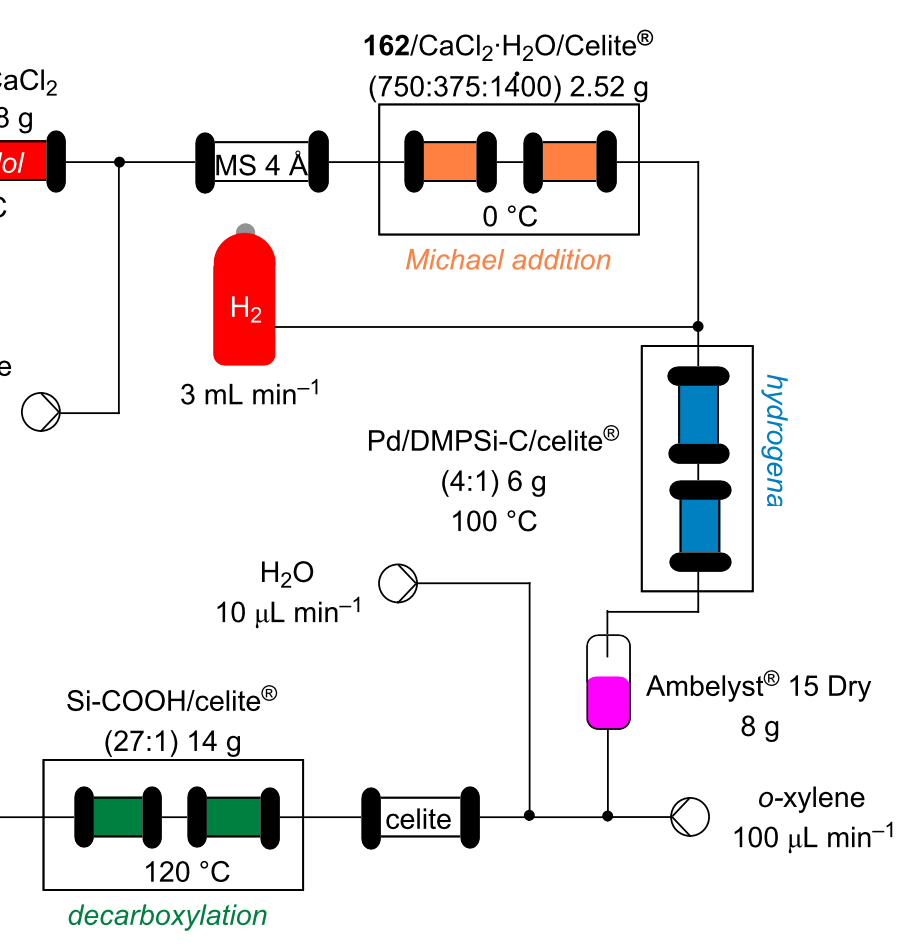

173, (R)-rolipram

Scheme 38: Continuous multistep synthesis for the preparation of $(R)$-rolipram (173). Si- $\mathrm{NH}_{2}$ : primary amine-functionalised silica gel.<smiles>Cc1cc2ccccc2[nH]1</smiles>

174

$0.1 \mathrm{M}(\mathbf{1 7 4})$ in DCM/EtOH $(9: 1)$

175 (1 equiv)

$0.90 \mathrm{~mL} \mathrm{~min}^{-1}$<smiles>O=C1C=CCCC1</smiles>

175

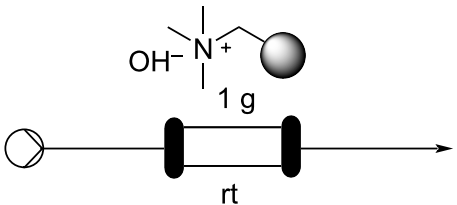

rt<smiles>Cc1[nH]c2ccccc2c1C1CCCC(=O)C1</smiles>

176

over $10 \mathrm{~h}$ runtime

$84-91 \%$ yield

Scheme 39: Continuous-flow Michael addition using ion exchange resin Amberlyst ${ }^{\circledR}$ A26.

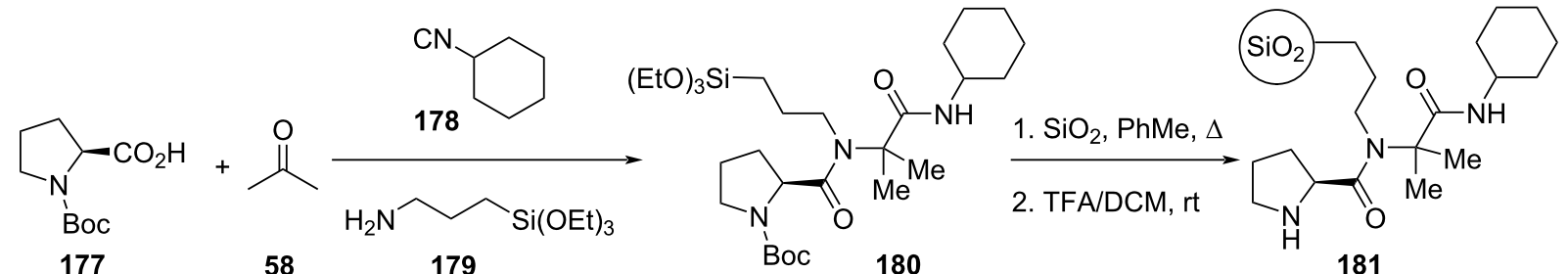

Scheme 40: Preparation of the heterogeneous catalyst 181 developed by Paixão et al. exploiting Ugi multicomponent reaction. 
Additionally, the authors applied a continuous-flow setup, which provides inline monitoring and product isolation (Scheme 41).

The system consists of two switching valves; the first one fills a $2 \mu \mathrm{L}$ sample loop, which is then sent to a chromatographic column packed with Boc-181 (red line), and the second one connects the latter output with a chiral column, which analyses the enantiopurity of the purified adduct 183 (green line) [199]. The system allows a significant reduction in the analysis and purification times. The flow system employing the immobilised catalyst 181 resulted stable for over 11 rounds $(\mathrm{TON}=304)$. The apparatus was also tested for the preparation of other 9 substrates, giving good to excellent yields (67-95\%) with high selectivity (syn/anti, 96:4, 82-92\% ee).

Using a different peptidic catalyst supported on polystyrene 184, Wennemers and Arakawa developed a flow system capable of preparing over $450 \mathrm{mmol}$ (>100 g) of Michael addition products in good to excellent yields (87-99\%) and high selectivity (syn/anti, 30:1, 94-97\% ee) using only $0.8 \mathrm{mmol}$ of catalyst $(\mathrm{TON}=610)($ Scheme 42$)[200]$.
Other polystyrene (PS)-supported catalysts for asymmetric conjugate additions have additionally been developed. PS-supported fluorinated proline $\mathbf{1 8 5}$, quinine $\mathbf{1 8 6}$, and squaramide 187 have all been prepared and exploited as organocatalysts by the group of Pericàs (Scheme 43) [201-204]. Interestingly, all the immobilised versions have demonstrated greater stability with lower deactivation rates when used in continuous-flow systems.

Wang and Zhang et al. applied PNAF-supported catalyst in flow for asymmetric conjugate addition of cyclohexanone $(\mathbf{6 5})$ and trans- $\beta$-nitrostyrenes (e.g., 74) [205]. The PNAF enables the use of greener solvents such as water and ethanol in the Michael reaction and creates a stable and robust heterogeneous catalyst which can be used over 4 cycles with comparable results (Scheme 44). The authors also claimed better outcomes using the flow approach compared to batch $(68 \%$ in $12 \mathrm{~h}$ vs $63 \%$ in $24 \mathrm{~h}$ ), which could be due to the higher effective concentration of the catalyst.

In 2019, Pericàs, Kappe and Ötvös adopted a polymer-supported organocatalyst $\mathbf{1 9 1}$ for the multistep synthesis of

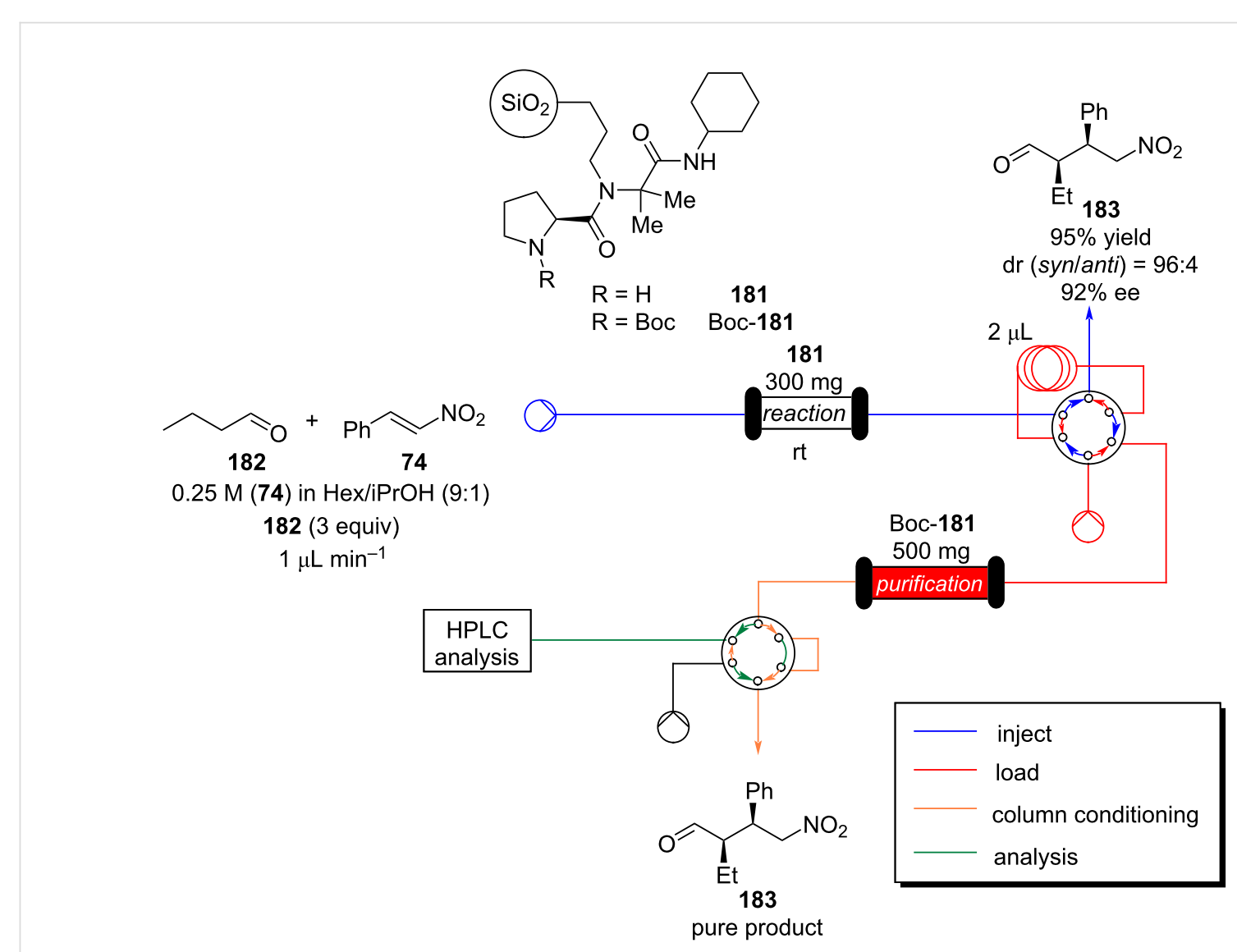




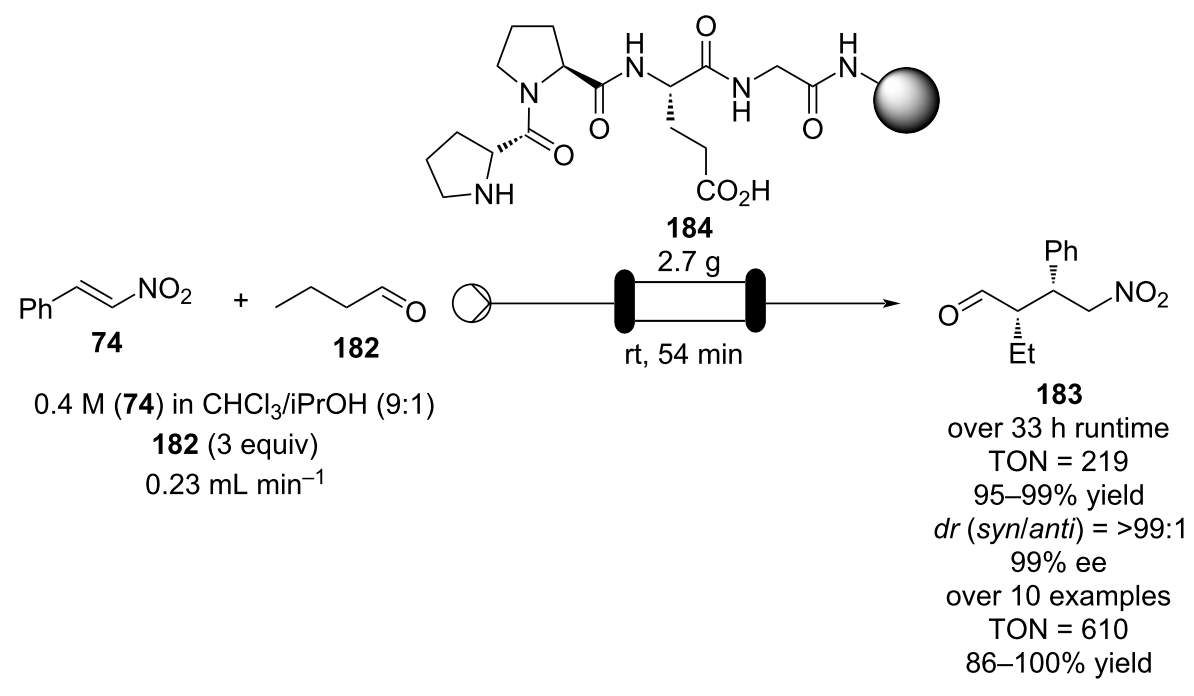

Scheme 42: Continuous-flow synthesis of nitroaldols catalysed by supported catalyst 184 developed by Wennemers and Arakawa.

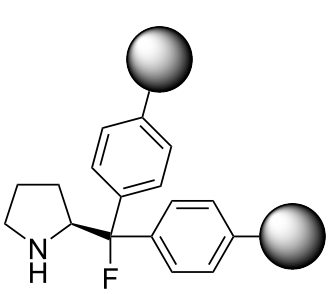

185

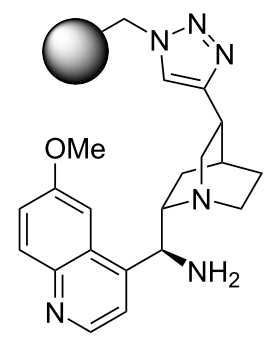

186

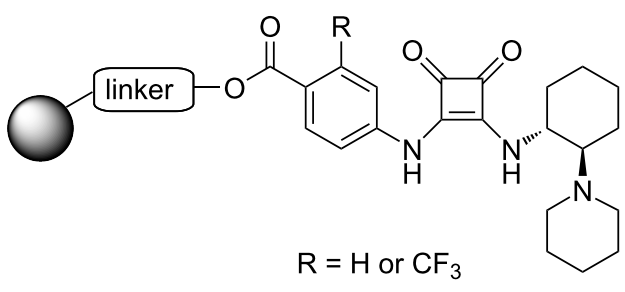

187

Scheme 43: Heterogenous polystyrene-supported catalysts developed by Pericàs and co-workers.

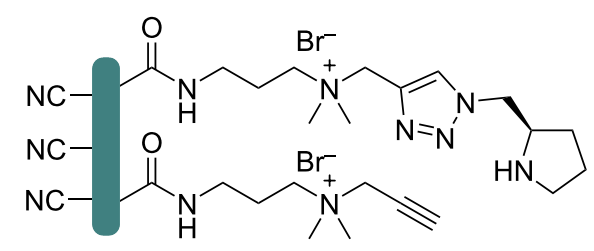<smiles>O=C1[CH+]CCCC1</smiles>

65

74

$0.05 \mathrm{M} \mathrm{(74)}$ in $\mathrm{H}_{2} \mathrm{O} / \mathrm{EtOH}(1: 1)$

65 (1 equiv)

$0.028 \mathrm{~mL} \mathrm{~min}^{-1}$

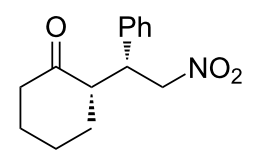

189

over 4 cycles

$63-68 \%$ yield $d r($ syn/anti $)=97: 3$

$99 \%$ ee

Scheme 44: PANF-supported pyrrolidine catalyst for the conjugate addition of cyclohexanone (65) and trans- $\beta$-nitrostyrene (74) in flow.

(-)-paroxetine precursor 195, a serotonin uptake inhibitor (Scheme 45) [206]. The synthesis was developed starting from dimethyl malonate (130) and 4-fluorocinnamaldehyde (190) for the synthesis of the intermediate 192. The such-developed catalyst delivers the desired material in good yield (84\%) with a high productivity $\left(2.47 \mathrm{~g} \mathrm{~h}^{-1}\right)$. The flow system developed for 


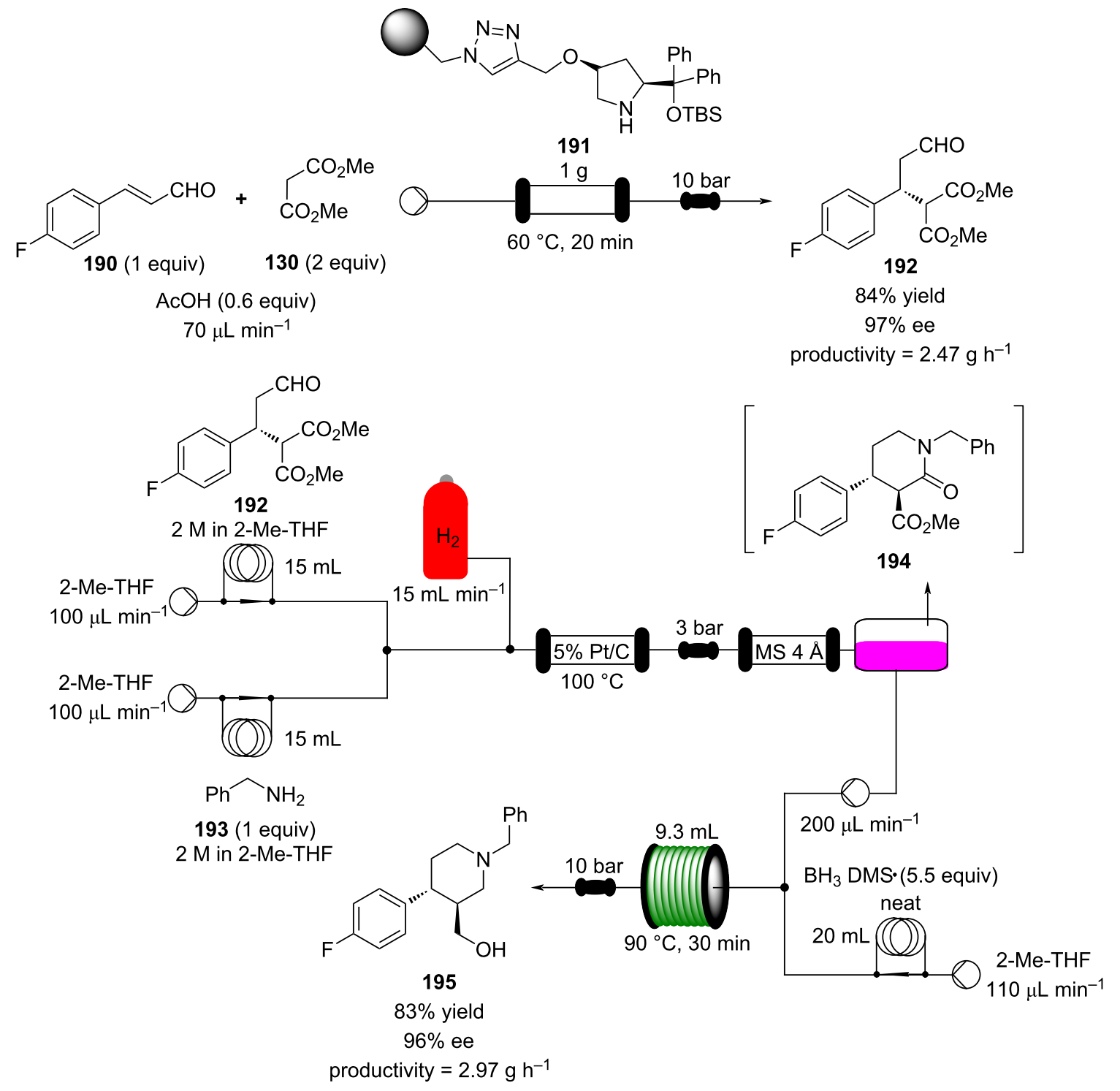

Scheme 45: Synthesis of (-)-paroxetine precursor 195 developed by Ötvös, Pericàs, and Kappe.

the conjugate addition operates in solvent-free conditions (SFC), therefore is isolated from the subsequent two steps of the multi-synthesis. A solution of $\mathbf{1 9 2}$ in 2-methyltetrahydrofuran (2-Me-THF) was then mixed with benzylamine for reductive amination to form the cyclic amide 194. Further reduction of the both amide and ester groups with borane dimethyl sufide $\left(\mathrm{BH}_{3} \cdot \mathrm{DMS}\right)$ allowed to isolate the desired material 195. With this two-steps continuous-flow system, $4.95 \mathrm{~g}$ of pure (-)-paroxetine were isolated in 100 minutes of collection (productivity $=2.97 \mathrm{~g} \mathrm{~h}^{-1}$ ).

Other examples utilising homogenous catalysts have also been shown to be effective in flow such as in the synthesis of (-)-oseltamivir (201) by Hayashi and Ogasawara (Scheme 46) [207]. The flow system is divided in 5 units comprising the 5 reaction steps: conjugate addition (red reactor), Michael addition and intramolecular Horner-Wadsworth-Emmons reaction (yellow reactor), nitrate anion protonation (blue reactor), epimerization (green reactor), and reduction (pink reactor). The apparatus employs a Comet-X-01 micromixer for better mixings, Teflon ${ }^{\circledR}$ tube reactors, and one Biotage SNAP empty cartridge. After 310 minutes of collection, the mixture was manually extracted and purified through standard column chromatography to gain $\mathbf{2 0 1}$ in $13 \%$ overall yield. The result is comparable to the one-pot batch procedure previously described (14\%) [208]. 


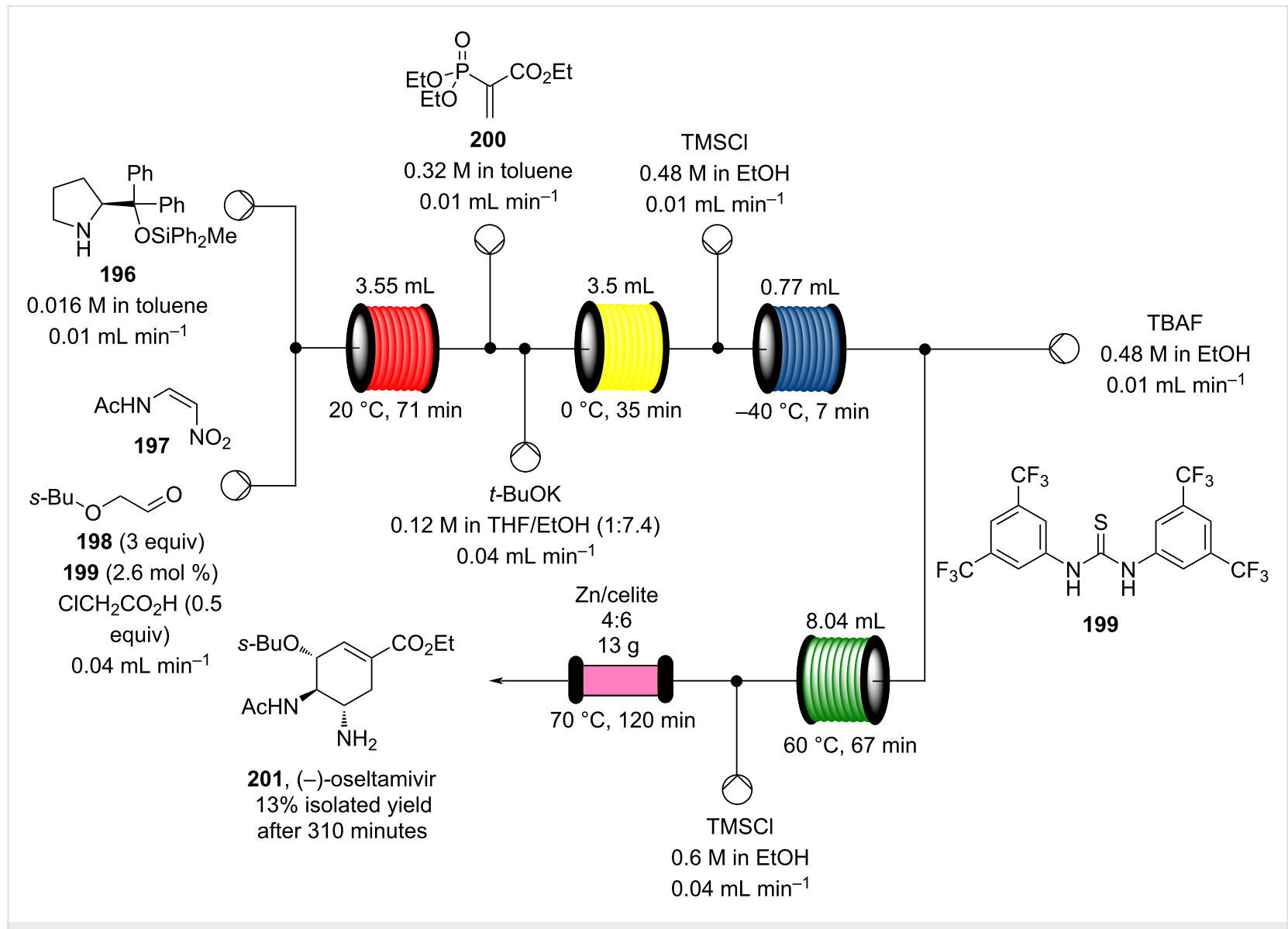

Scheme 46: Continuous-flow approach for the 5-step synthesis of (-)-oseltamivir (201) as devised by Hayashi and Ogasawara.

In 2014, a continuous enzyme-catalysed conjugate addition system was reported by Du et al. [209]. Using silica-absorbed Lipozyme ${ }^{\circledR}$ as the catalyst, the authors managed to synthesise twelve adducts between various pyrimidine derivatives (5-fluorouracil, uracil, and thymine) and acrylate acceptors with a reduction in the reaction times compared to the batch reaction (Scheme 47).
Other types of conjugate addition processes have also been performed within continuous-flow systems, for example, 1,6conjugate addition to para-quinone methide using either thiols [210] or alkylzinc compounds [211]. A continuous-flow coppercatalysed 1,4-conjugate addition to $\alpha, \beta$-unsaturated carbonyls has been developed by Ley et al. [212]. For this example, the commercial flow platform Vapourtec E-series was employed.

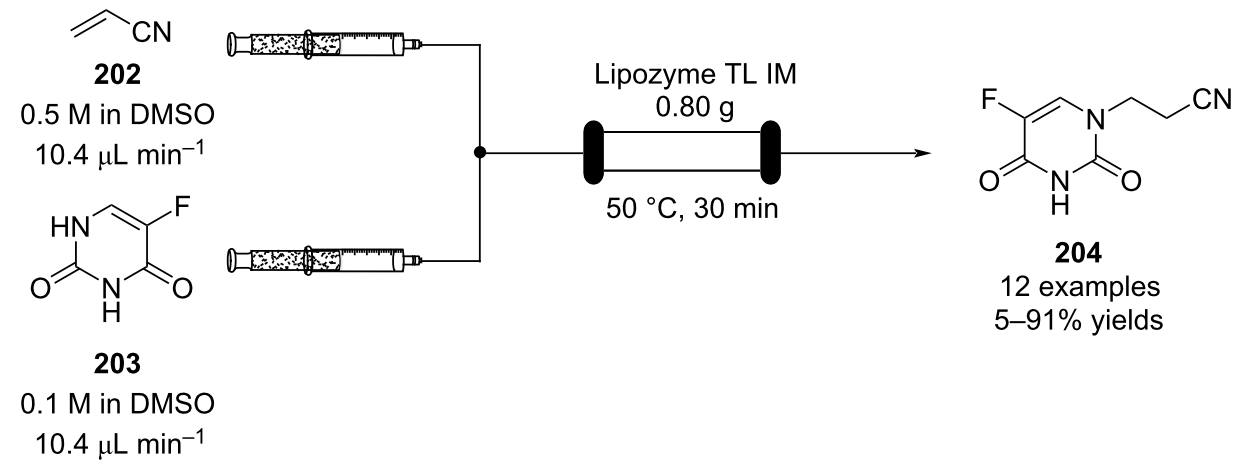


As organometallic compounds are moisture sensitive, the choice of the pumping system was crucial in order to avoid quenching or long-term stability issues. Alternative HPLC-type pumps are more prone to fouling and blocking, due to precipitation of hydrolytic materials. On the other hand, peristaltic pumps, in which the fluid is contained within a tube and wetted parts are thus minimised at the pump, allows better long term performance and reduces maintenance. Thus, in the work of Ley et al better results in terms of regioselectivity 1,4:1,2 were obtained compared to the corresponding batch process (99:1 vs 1:1), which also required lower temperatures $\left(-60^{\circ} \mathrm{C}\right.$ vs $\left.-10^{\circ} \mathrm{C}\right)$ and longer reaction time ( 2 hours vs $\approx 6$ minutes) (Scheme 48 ) [213]. To highlight the reproducibility of the system, a second operator was asked to optimise the same experiment and essentially identical outcomes were obtained.

\section{Hydrogenation}

The reduction of alkynes to alkenes/alkanes, alkenes to alkanes and ketones/aldehydes to alcohols are important transformations producing countless valuable products for which catalysed hydrogenation represents a key process. Alkyne hydrogenation is particularly relevant to the synthesis of monoterpene derivatives, for example, 6-methyl-5-hepten-2-one (210) [214], itself a fragrance ingredient but also a precursor to many others, which is prepared from the hydrogenation product $\mathbf{2 0 9}$ of 2-methylbut3-yn-2-ol (208) [215,216]. Other examples include the synthe- sis of cis-jasmone (212) [217] and methyl jasmonate (12) which requires hydrogenation over Lindlar's catalyst [218]. Examples of alkene hydrogenation include the reduction of $\alpha$-pinene (213) to cis-pinane (214) [219], a useful intermediate in the preparation of linalool (10) and the aromatic reduction of catechol derivative 215, to prepare isocamphyl cyclohexanol (216) [220], a synthetic sandalwood equivalent. Also, the hydrogenation of L-isopulgol to L-menthol (L-218) [221] and menthone/isomenthone $\mathbf{2 1 7}$ to menthol/isomenthol diastereomers 218 [222] are all important and commonly used transformations (Scheme 49).

Improvements in safety, performance and environmental impact can be expected when carrying out hydrogenation reactions under flow conditions [223]. Batch hydrogenation processes are often limited by gas solvent diffusion rates (interfacial contact having a large impact). The implementation of flow technology allows for the rapid saturation of reaction media by making use of biphasic in-line mixing or gas-permeable membranes, leading to improved reaction kinetics. Devices such as the ThalesNano H-Cube ${ }^{\circledR}[118,224-233]$ (Figure 7) are used to effect highly efficient in-line mixing of hydrogen on a laboratory scale. Hydrogen is generated from electrolysis of water giving oxygen (or ozone) as a byproduct. The hydrogen gas is sparged into the liquid flow stream, creating bubble or pipe flow. A variety of semi-disposable catalyst cartridges can be incorporated into the system which minimise user contact with

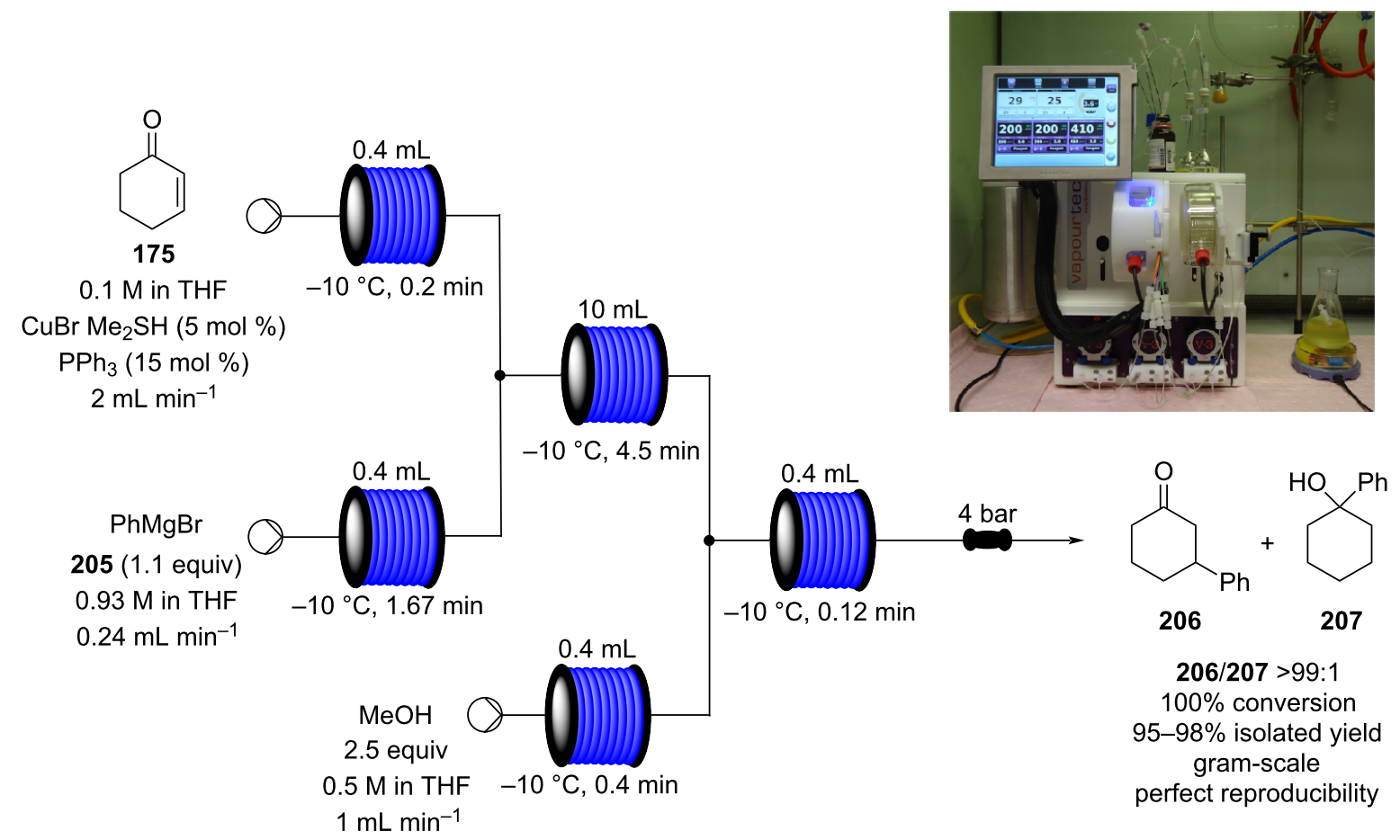

Scheme 48: Continuous-flow copper-catalysed 1,4 conjugate addition of Grignard reagents to enones. Reprinted with permission from [212]. Copyright 2013 American Chemical Society. 


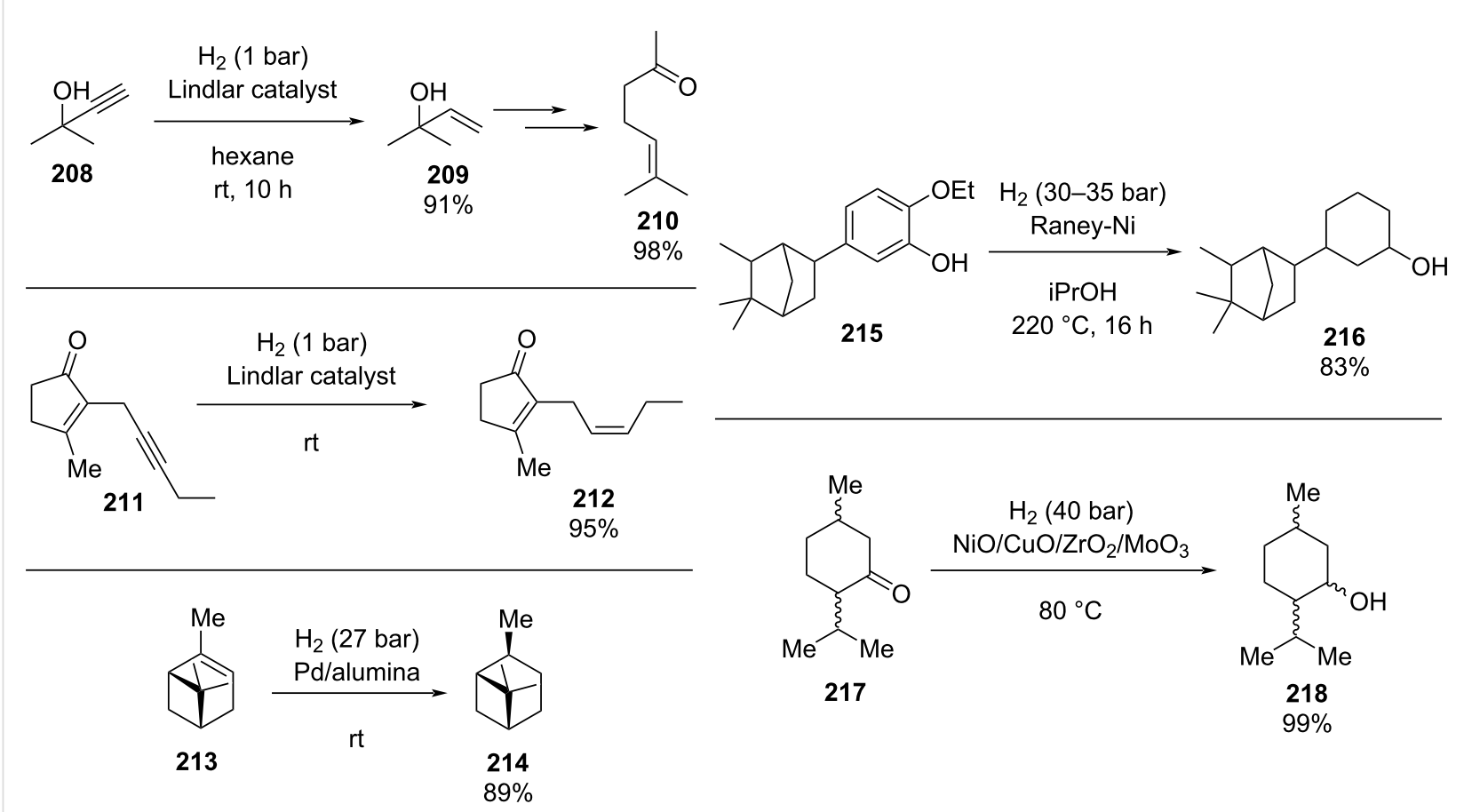

Scheme 49: A collection of commonly encountered hydrogenation reactions.

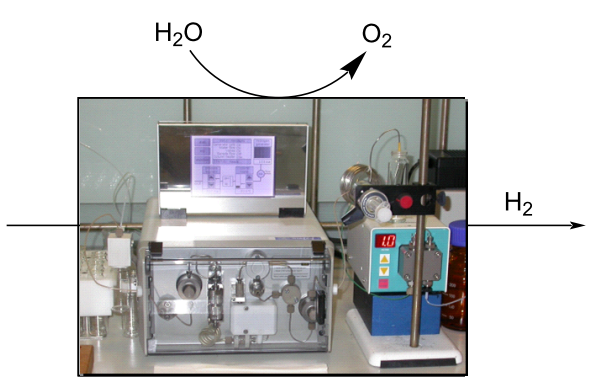

Figure 7: The ThalesNano H-Cube ${ }^{\circledR}$ continuous-flow hydrogenator. potentially toxic and pyrophoric materials (i.e., Raney nickel) and facilitate a broad range of transformations.

The convenience of the H-Cube ${ }^{\circledR}$ with the facility to swap catalyst cartridges offers an attractive approach. In 2011, it was reported for the chemoselective reduction of an $\alpha, \beta$-unsaturated ketone 78 [118]. The reduction could be controlled for the ketone 85 by using either a $\mathrm{Pd} / \mathrm{C}$ cartridge at $70{ }^{\circ} \mathrm{C}$ or a Raney nickel cartridge at room temperature or, alternatively, the reaction can be progressed through to the alcohol 219 under harsher conditions (Scheme 50).

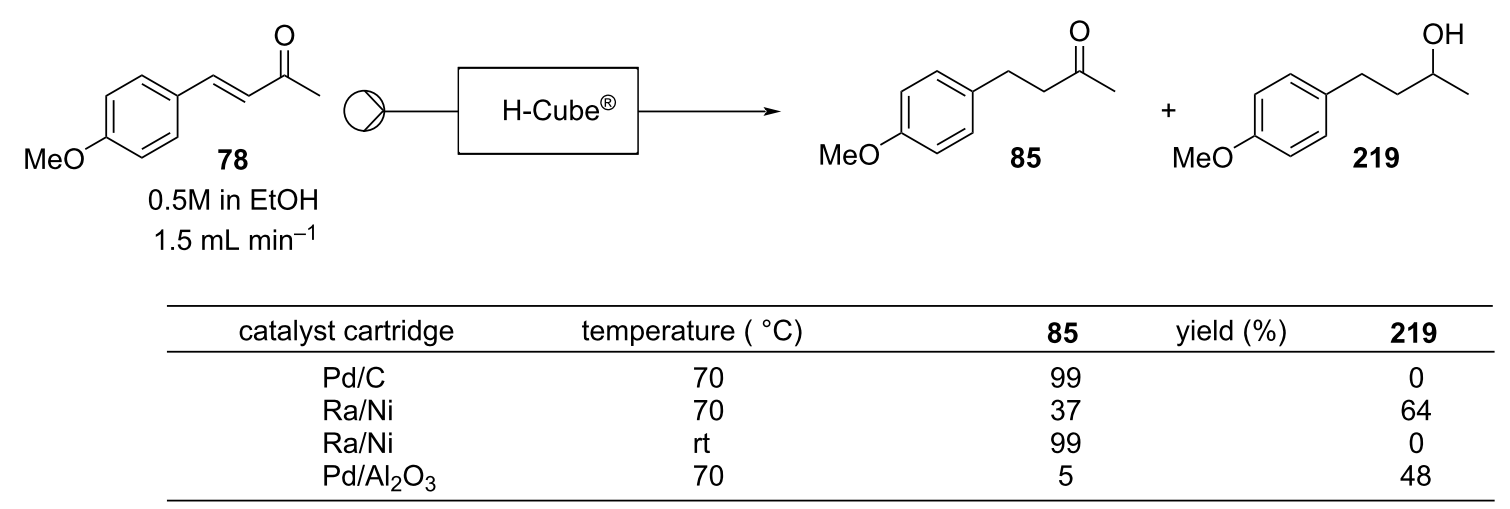


The chemoselective reduction of alkynes to alkenes has also been achieved in flow with a Lindlar catalyst cartridge (Scheme 51) [234]. The product, 220, of a Marshall homopropargylation flow procedure was subjected to hydrogenation following dissolution in EtOAc to effect partial reduction of the triple bond, yielding the olefin $\mathbf{2 2 1}$ in $95 \%$ yield with a high diastereomeric ratio (4.3:1). The standard conditions were $25^{\circ} \mathrm{C}$ at 10 bar using a $\mathrm{Pd} / \mathrm{BaCO}_{3} / \mathrm{PbO}$ catalyst cartridge. However, by increasing the pressure to 30 bar it was possible to carry out the transformation in $\mathrm{CH}_{2} \mathrm{Cl}_{2}$.

Recently, the H-Cube ${ }^{\circledR}$ reractor has also been employed for the semi-hydrogenation of intermediate 208 using single-atom heterogeneous catalysts (SACs) [235-238]. These catalysts, developed by Pérez-Ramírez and co-workers, consist of atomically dispersed noble metals on an organic host, usually nitrogen-enriched carbon materials. The catalysts are mixed over silicon carbide (SC), placed in a cartridge, and then tested in the H-Cube ${ }^{\circledR}$ apparatus (Scheme 52). The catalysts were highly selective (96-99\%) and reusable (up to 50 hours of runtime), however, low conversions are still recorded $(25-50 \%)$.

In addition, many other diverse reduction processes have been effected by the H-Cube ${ }^{\circledR}$ system. It has been utilised for the reduction of amides [239], nitro compounds [240-242], nitriles [243-245], azides [246,247], diazo compounds [248], and for carrying out reductive aminations [249-251] on a laboratory scale, while the $\mathrm{H}-\mathrm{Cube}{ }^{\circledR}$ midi, has been designed specifically to be used in larger scale processes [252-254]. Recently, the
H-Cube ${ }^{\circledR}$ apparatus has also found use in the evaluation for biomass-derived chemicals recovery, such as Ru-catalysed hydrogenation of methyl levulinate (262) from lignocellulosic biomass to $\gamma$-valerolactone (263) [255], and scrap waste recovery, such as the scrap ceramic-cores of automotive catalytic converters (CATs) employed in the chemoselective hydrogenation of cinnamaldehyde to hydrocinnamyl alcohol [256,257].

Alternatively, tube-in-tube gas-liquid reactors [258,259] have become popular to facilitate efficient mixing in flow by enabling pressurised gas permeation across a membrane into a coaxial liquid flow. The most common design comprises a coiled non-permeable outer tube (typically stainless steel, polytetrafluoroethylene or PTFE) under positive gas pressure and an inner gas-permeable tube (Teflon $\left.{ }^{\circledR} \mathrm{AF}-2400\right)$ that thus allows the gas to diffuse from the outer tube to the inner tube. The high surface area of the permeable tube enables higher gas-liquid contact (Figure 8), and leads to rapid, highly efficient gas saturation of the reaction medium.

Both approaches have been used with great success in recent years, with the latter being used for several other gaseous reactions including ozonolysis, carbonylations, carboxylations, reactions requiring ammonia- and oxygen-mediated reactions (increasingly in combination with photochemistry) as well as hydrogenations [17]. In many instances, telescoping of reaction steps is entirely possible and this is indeed being illustrated by their increasing incorporation into unified multistep total syntheses [260-264].

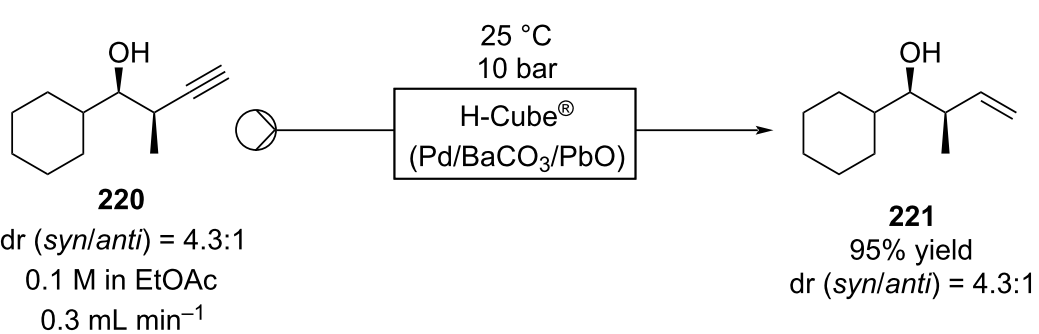

Scheme 51: Incorporation of Lindlar's catalyst into the $\mathrm{H}$-Cube ${ }^{\circledR}$ reactor for the reduction of an alkyne.

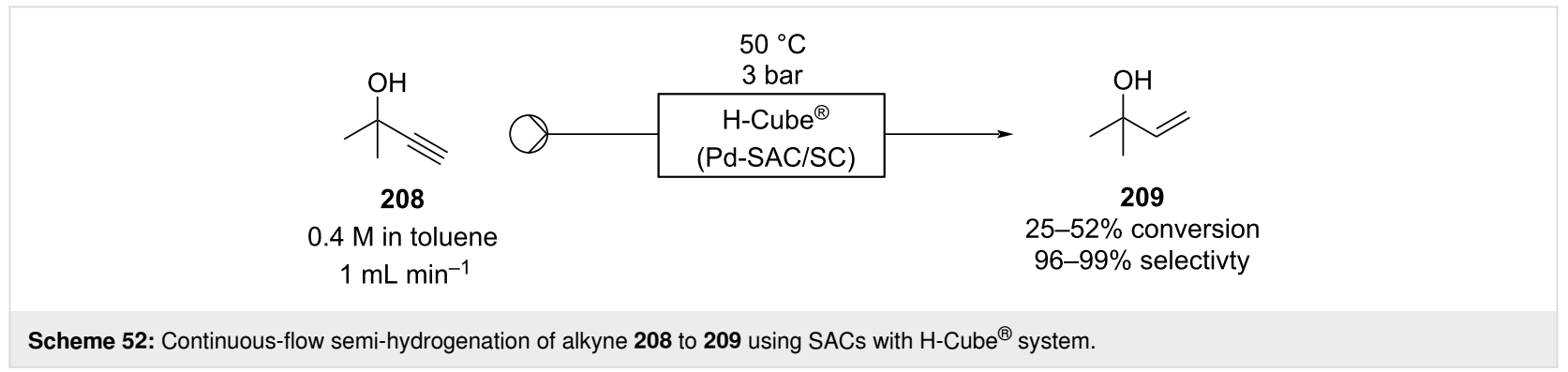



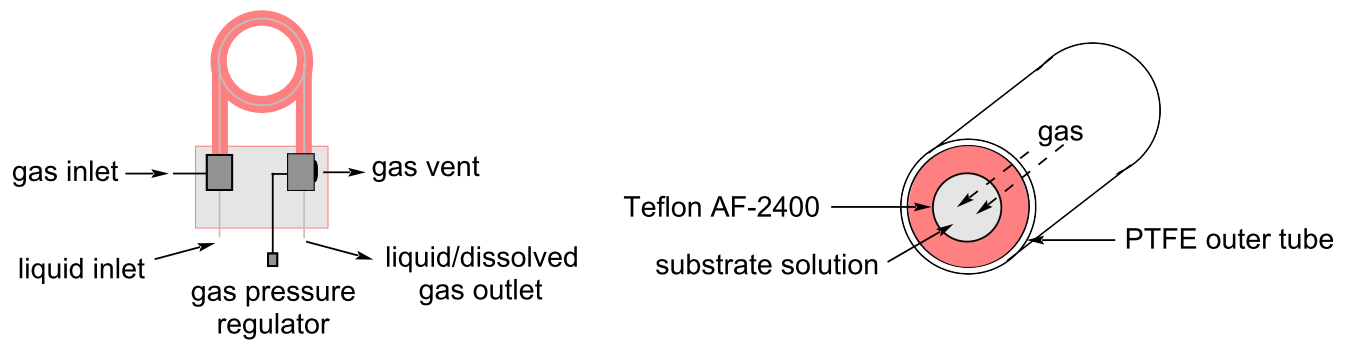

Figure 8: The standard setups for tube-in-tube gas-liquid reactor units.

In 2011, the tube-in-tube reactor was used for the hydrogenation of a range of olefins in flow by the Ley group [265]. Crabtree's catalyst $(0.1 \mathrm{~mol} \%)$ was used (injected as a separate flow stream via an injection loop) to study the hydrogenation of ethyl cinnamate (197) under continuous hydrogenation conditions (Scheme 53 ). The conversion was found to be highly dependent on the applied hydrogen pressure $(\approx 50 \%$ at 10 bar vs $100 \%$ at 30 bar). Having highly precise pressure control, coupled with a large temperature window are significant advantages of the tube-in-tube reactors. The methodology was applied to seven other structures vaguely reminiscent of fragrances/fragrance intermediates and quantitative yields were obtained in each case after rapid optimisation.

A heterogeneous hydrogenation protocol, utilising a Pd/Cpacked catalyst cartridge, was also developed (Scheme 54) and applied to a further 8 substrates with all being obtained in quantitative yields (Table 6). Here, a recycling approach was taken, ensuring that all starting materials reached full conversion, the number of passes necessary varied according to the substrate (alkyne 230, for example, took much longer than all others $\approx 250$ min).
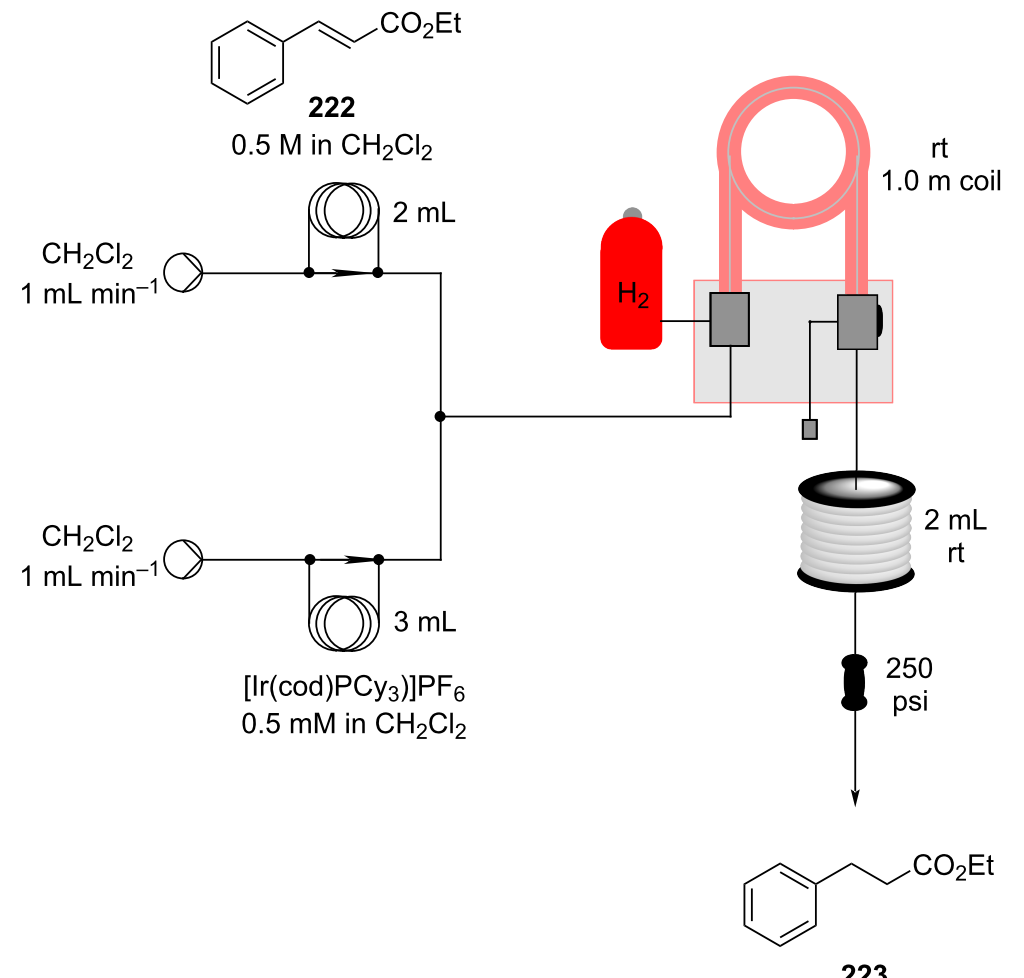

8 examples, all quantitative 


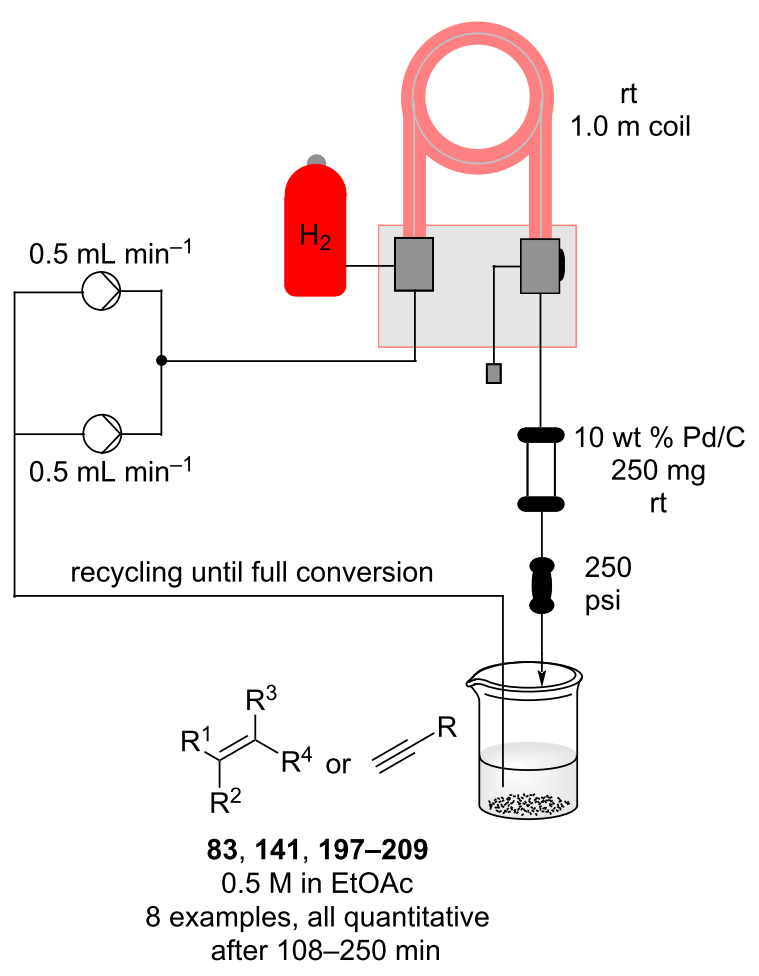

Scheme 54: Recyclable heterogeneous flow hydrogenation system.

Table 6: Substrate screening of the recyclable heterogeneous flow hydrogenation system.

\begin{tabular}{|c|c|c|c|c|}
\hline Entry $^{a}$ & Substrate & Product & Time (min) & Yield (\%) \\
\hline 1 & 222 & 223 & 125 & quant. \\
\hline 2 & 224 & 225 & 130 & quant. \\
\hline 3 & 90 & 151 & 120 & quant. \\
\hline 4 & 226 & 227 & 115 & quant. \\
\hline 5 & 228 & 229 & 120 & quant. \\
\hline 6 & 230 & 225 & 250 & quant. \\
\hline
\end{tabular}


Table 6: Substrate screening of the recyclable heterogeneous flow hydrogenation system. (continued)

7<smiles>CC(=O)C=Cc1ccc(F)cc1</smiles>

231

8<smiles>C=C(CC(C)(C)c1ccccc1)c1ccccc1</smiles><smiles>CC(=O)CCc1ccc(F)cc1</smiles>

232<smiles>CC(CC(C)(C)c1ccccc1)c1ccccc1</smiles>

234
108

quant.

quant.

aReactions conducted on $5 \mathrm{mmol}$ scale.

In a similar approach, the Leadbeater group investigated the incorporation of Wilkinson's hydrogenation catalyst in a reverse tube-in-tube reactor (gas permeation from inner to outer tubing) to effect reduction of a set of 11 compounds (Scheme 55) [259]. A scale-up operation for the hydrogenation of chalcone (235) gave a productivity of $9 \mathrm{~g} \mathrm{~h}^{-1}$, equating to roughly $227 \mathrm{~g} \mathrm{~d}^{-1}$.

To achieve better gas-liquid-solid interaction, microreactors are a good choice for small scale processes. As an example, Kobayashi's group performed a microencapsulation of Pd to immobilise the metal on the glass microchannel walls within the reactor (a microchannel reactor 'MCR') [266]. The authors pointed out the importance of the flow rates which affected contact times and thus overall conversions. As shown in Scheme 56, a thin film of liquid wets the microreactor's coated wall where the catalyst was deposited, whereas the less dense hydrogen flows through the centre (an example of pipe flow). The same group also employed the reactor for use with $\mathrm{scCO}_{2}$ [267].

A different method for surface contact enhancement was explored by Kreutzer et al., who developed a hydrogenating system using segmented flow (Scheme 57) [268].

The internal glass capillary tubes were coated with alumina and treated to deposit a layer of Pd nanoparticles. The authors employed a segmented gas-liquid flow where alternating bubbles of hydrogen and substrate are generated inside the capillary. The hydrogen consumption could be visually followed from the decrease in size (volume) of the gas bubble as it progresses along the tube. The system was applied to the semi-hydrogenation of 3-methyl-1-pentyn-3-ol (238) reaching a theoretical

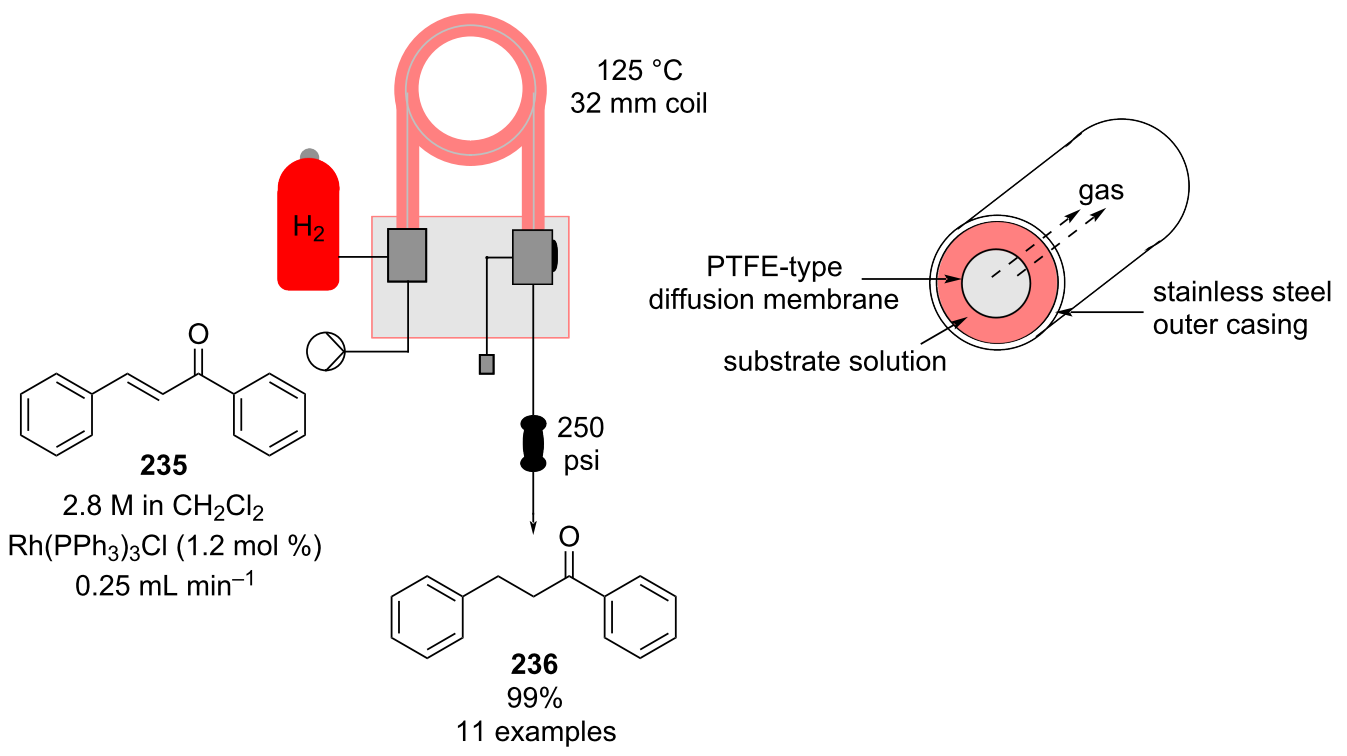

Scheme 55: Leadbeater's reverse tube-in-tube hydrogenation system for olefin reductions. 
<smiles>CC(=O)/C=C/c1ccccc1</smiles>

$0.1 \mathrm{M}$ in THF $0.1 \mathrm{~mL} \mathrm{~h}^{-1}$

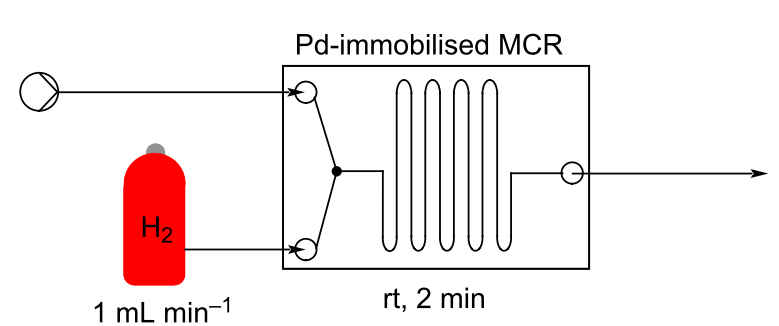

$1 \mathrm{~mL} \mathrm{~min}^{-1}$

rt, 2 min<smiles>CC(=O)CCc1ccccc1</smiles>

$97 \%$ yield

11 examples

b)

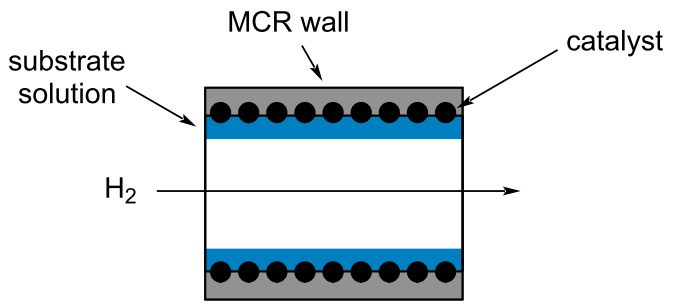

Scheme 56: a) Hydrogenation using a Pd-immobilised microchannel reactor (MCR) and b) a representation of the ideal conditions for an optimum interphase system.

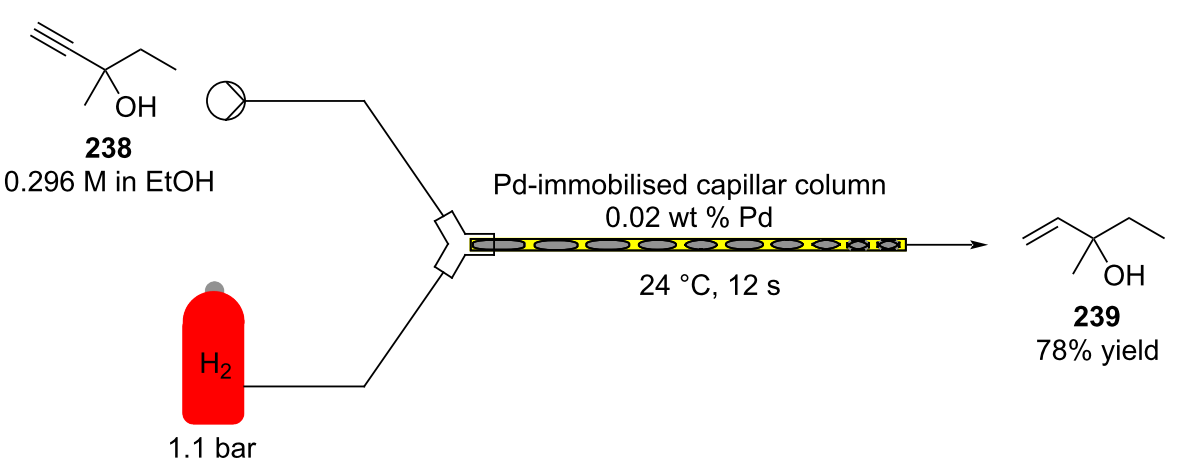

Scheme 57: Hydrogenation of alkyne 238 exploiting segmented flow in a Pd-immobilised capillary reactor.

optimum yield around $78 \%$. The authors also claimed lower outcomes were obtained without the use of segmented flow $(57 \%)$.

Recently, a Vortex fluidic device (VFD) has emerged as a promising process intensification tool. The apparatus consist of a fast rotating surface that creates dynamic thin films which generates a high mass and heat transfer regime. Several examples have already been setup to perform different chemical transformations [269]. In 2019, the group of Chalker and Raston utilised an VFD-based hydrogenating system demonstrating sucessful reduction of nitro groups and alkenes [270]. A catalyst system comprising Pd nanoparticles dispersed on cellulose paper was easily prepared and could be reusable over 10 times without deactivation. The apparatus was also explored for the continuous hydrogenation of (-)-levoglucosenone (product 240) (Scheme 58) and reduction of isophorone (product 242) in $93 \%$ conversion after recycling the solution three times back through the device. The VFD has also found utility in transfer hydrogenations using ammonium formate, hydrogenated cinnamic acid (243) was detected in $89 \%$ conversion after the third cycle.

A valuable alternative to the above described apparatus has been developed by Hornung et al. The authors addressed the need for efficient mixing against the drawback of the many common catalyst supports; high pressure drops (small particle/ high surface area), brittleness, inhomogeneity, and often long complex preparations. The flow system selected adopted a tubular reactor filled with catalytic static mixers (CSM's) [271]. These static mixers were coated with metals such as nickel, palladium, or platinum through either electroplating or cold spraying. The supporting matrix were made from robust metallic alloys ( $\mathrm{Ti}, \mathrm{CoCr}$, and stainless steel) and manufactured using 3D printer techniques. The CSM designs enhance the biphasic mixing ensuring a high interfacial contact area. Such 

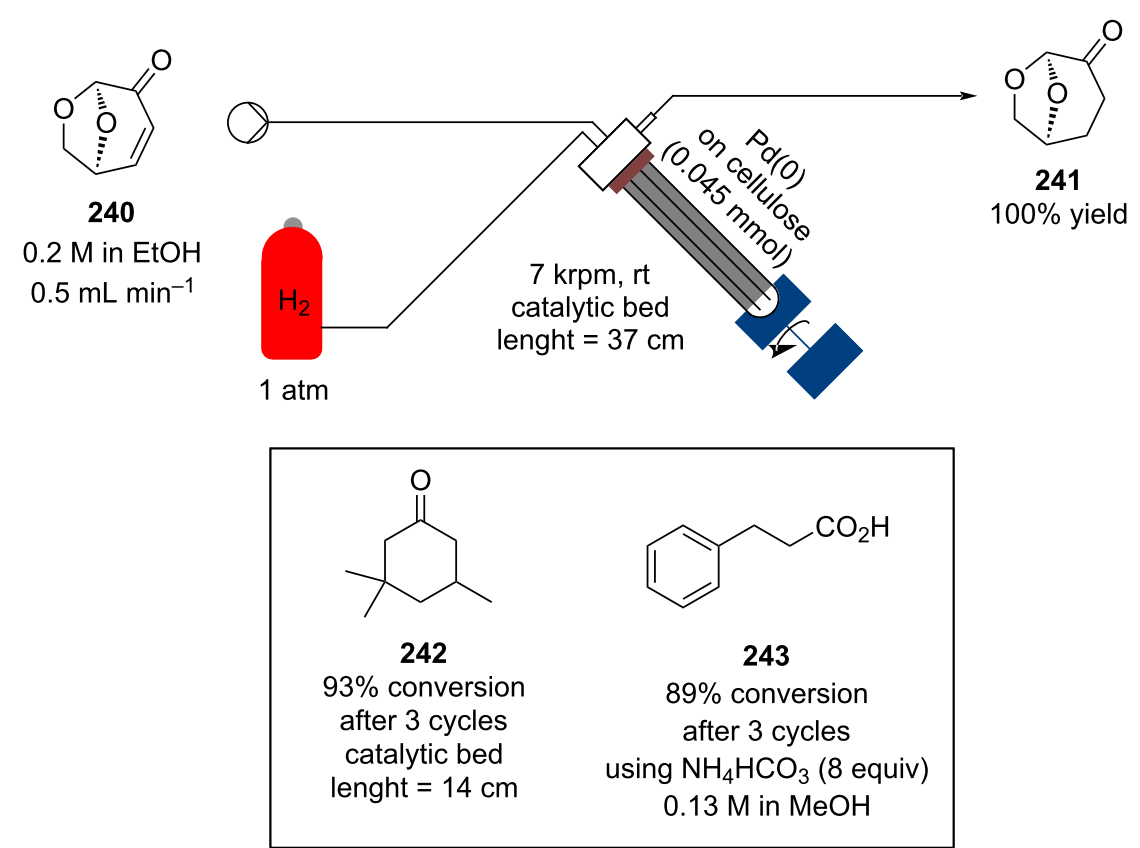

Scheme 58: Continuous hydrogenation system for the preparation of cyrene (241) from (-)-levoglucosenone (240).

system has been investigated for the reduction of alkenes, alkynes, ketones, diazo-, nitro compounds, nitriles, imines, and aryl halides [272]. In all the investigated examples, the desired products were detected in moderate to excellent conversions (50-100\%). Furthermore, the group also optimised various hydrogenation processes of industrial interest such as the hydrogenation of vinyl acetate and synthesis of $\mathbf{2 2 5}$; a key intermediate for linezolide (251), a valuable last-line-of-defence antibiotic (substructure depicted in red, Scheme 59) [273,274].

In 2013 a selective flow reduction of ketones over aldehydes using direct in-line $\mathrm{H}_{2}$ mixing was reported [275]. The substrates 252 comprised both aldehyde and ketone functionalities and it was found that, by initial acetalisation of the more reactive aldehyde moiety, the ketone could be selectively hydrogenated, leaving the masked aldehyde intact (Scheme 60). A Ti(IV) cation exchanged montmorillonite (Ti-mont) was used as a heterogeneous acid catalyst for the acetalisation/deacetalisation and a hydroxyapatite (HAP)-supported Ru nanoparticle catalyst packed reactor was used for the hydrogenation, both operated at slightly elevated temperatures.

Other examples of heterogeneous catalysts used for generating telescoped multistep synthesis have been reported. For example, Pd-immobilised silica monoliths have been exploited in continuous hydrogenation [276,277]. The isotropic network created by the monolith allows the preparation of highly dispersed catalysts with good mechanical properties and catalytic activities
[7]. In 2013, Galarneau et al. used such an immobilised Pd system for the semi-hydrogenations of 1,5-cyclooctadiene (COD, 256) and 3-hexyn-1-ol (258). The system provided a constant $95 \%$ conversion and $90 \%$ selectivity to the corresponding monoalkene 257 over 70 hours of runtime, and $85 \%$ conversion, $70-80 \%$ selectivity for the hydrogenation product $\mathbf{2 5 9}$ over 7 hours (Scheme 61) [277]. The authors claimed the selectivity to $\mathbf{2 5 9}$ could be improved via a silanol group deactivation treatment of the support.

Further examples of semi-hydrogenation using supported Pd nanoparticles have also been developed by Barbaro et al. A titanate nanotube (TiNT) support was explored, with the catalyst being prepared through an ion-exchange/in situreduction process. This allowed for the generation of welldispersed nanoparticles with narrow diameter distribution, essential to the selectivity of the catalyst system. As such compound 259 was obtained at $83 \%$ conversion and $82 \%$ selectivity $\left(\mathrm{TOF}=850 \mathrm{~h}^{-1}, \mathrm{STY}=0.83 \mathrm{~kg} \mathrm{~L}^{-1} \mathrm{~h}^{-1}\right)$ [278].

In 2019, the Vorholt and Leitner groups developed a mini-plant for continuous hydrogenation of the lignin-derived building block furfural acetone (260) to 2-butyltetrahydrofuran (261), a promising biodiesel fuel. The flow process consisted of two reaction steps: hydrogenation and hydrodeoxygenation. For the first step, a Ru/C catalyst was employed, whereas in the second step, a sulfonic resin (Amberlyst ${ }^{\circledR} 36$ ) was added as the acid catalyst (Scheme 62). The apparatus was operated efficiently 


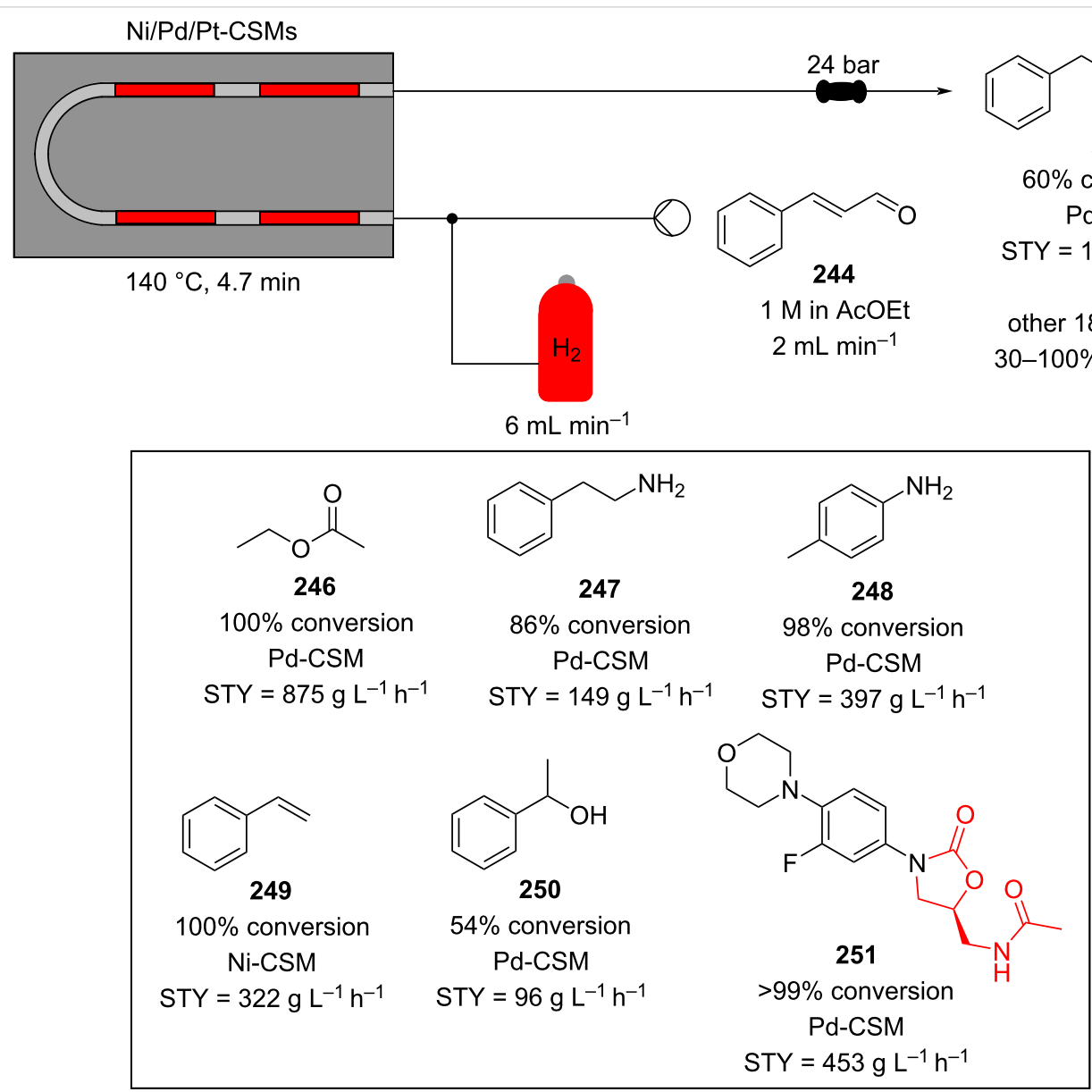

Scheme 59: Continuous hydrogenation system based on CSMs developed by Hornung et al.

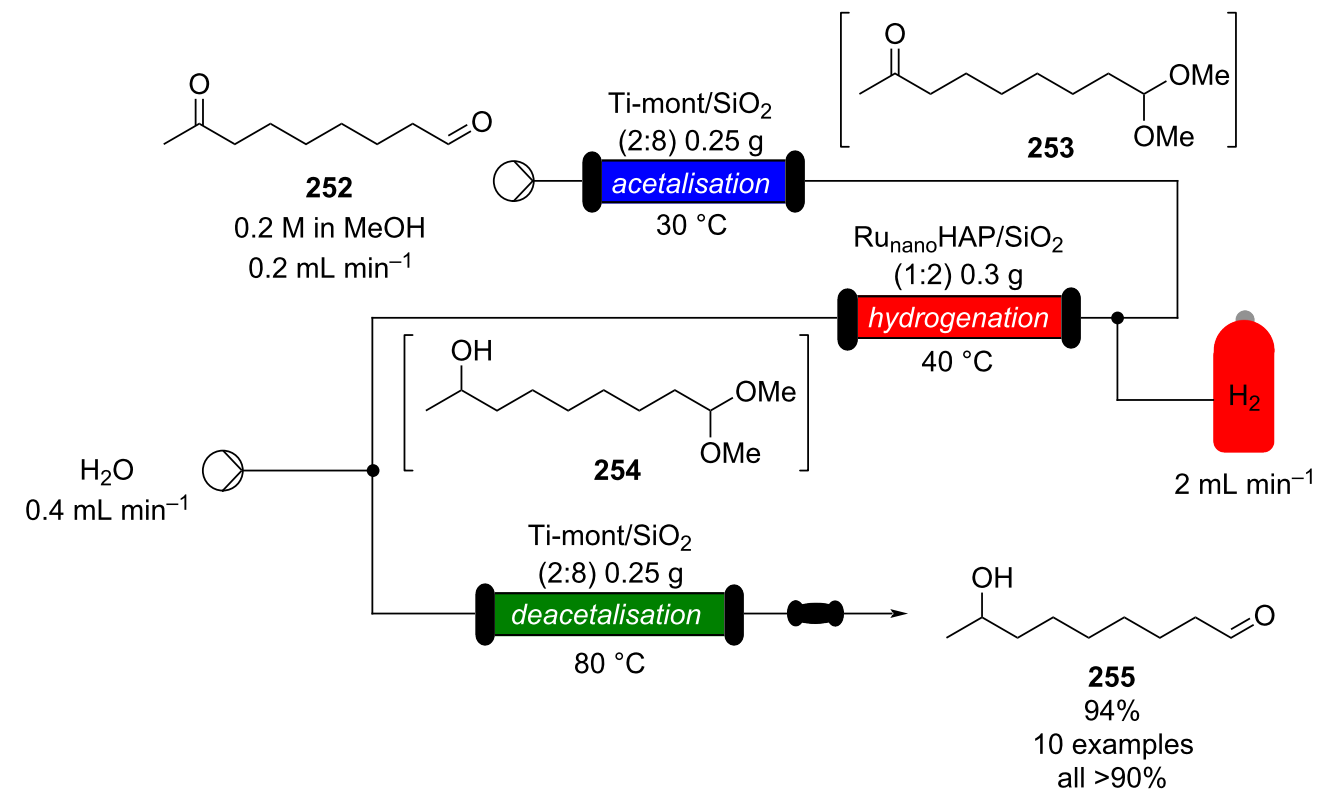

Scheme 60: Chemoselective reduction of carbonyls (ketones over aldehydes) in flow. 


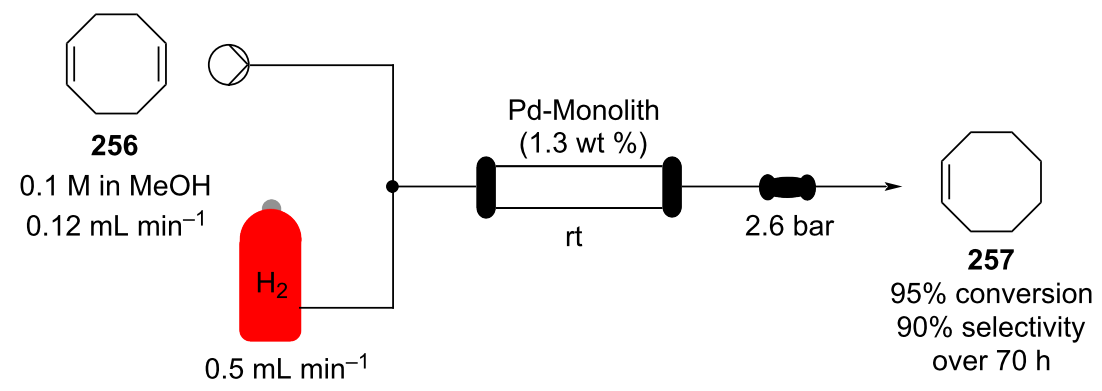

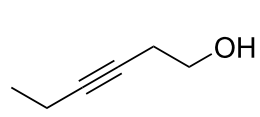

258

$0.2 \mathrm{M}$ in $\mathrm{MeOH}$

$0.15 \mathrm{~mL} \mathrm{~min}^{-1}$

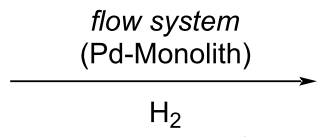

$0.7 \mathrm{~mL} \mathrm{~min}^{-1}$

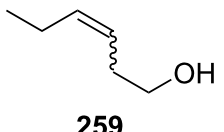

259

$85 \%$ conversion

$70-80 \%$ selectivity STY $=270 \mathrm{~g} \mathrm{~L}^{-1} \mathrm{~h}^{-1}$

Scheme 61: Continuous system for the semi-hydrogenation of 256 and 258, developed by Galarneau et al.

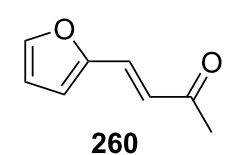

$0.25 \mathrm{M}$ in cyclohexane $1 \mathrm{~mL} \mathrm{~min}^{-1}$

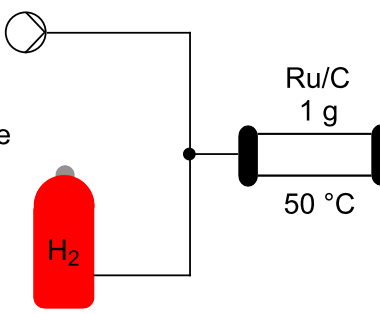

$50 \mathrm{~mL} \mathrm{~min}{ }^{-1}$
$\mathrm{Ru} / \mathrm{C} /$ Amberlyst $^{\circledR} 36$ $(1: 6) 7 \mathrm{~g}$

$150{ }^{\circ} \mathrm{C}$
80 bar

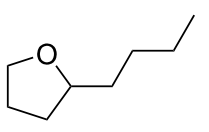

261

$86-87 \%$ yield

up to $5 \mathrm{~h}$

$75 \%$

up to $10 \mathrm{~h}$

Scheme 62: Continuous synthesis of biodiesel fuel $\mathbf{2 6 1}$ from lignin-derived furfural acetone (260).

for up to 5 hours, yielding 261 in a consistent $86-87 \%$. A slight decrease of yield to $75 \%$ was detected during the following 5 hours of runtime due to gradual catalyst deactivation [279]. The authors claimed the water formed during the sequence is absorbed onto the support, reducing the active surface area of the catalyst in the second step. Obviously, it would be relatively easy to build a secondary exchangeable cartridge into the system to allow cleaning and reactivation whilst maintaining continuous operation.

Pineda et al. optimised continuous-flow catalytic transfer hydrogenation (CTH) for the synthesis of $\gamma$-valerolactone (263) from lignin-derived methyl levulinate (262) in flow (Scheme 63) [280,281]. One of the systems operated with a mesoporous<smiles>CC(=O)CCC(C)=O</smiles>

262

$0.2 \mathrm{~mL} \mathrm{~min}^{-1}$

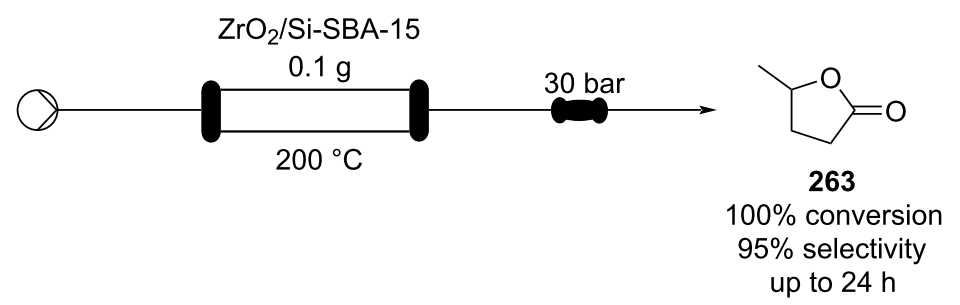

Scheme 63: Continuous synthesis of $\gamma$-valerolacetone (263) via CTH developed by Pineda et al. 
acidic material (Si-SBA-15) and $\mathrm{PrOH}$ as the hydrogen donor. When a stream of $\mathbf{2 6 2}$ is heated at $200{ }^{\circ} \mathrm{C}$ and passed over the catalyst $100 \%$ conversion and $95 \%$ selectivity to the desired product 263 was achieved. The catalyst was able to operate for over 24 hours with no substantial change in productivity giving a throughput of $30 \mathrm{mmol} \mathrm{g}^{-1} \mathrm{~h}^{-1}$ [280].

Recently, an alternative eco-friendly supported Ni-based catalyst has also been developed for the continuous hydrogenation of lignin-derived biomass sources such as glucose, xylose, and vanillin [282]. The heterogeneous catalyst was prepared from semolina, urea, glucose, and water to form after processing a nitrogen-doped carbon (NDC) support. The nickel nanoparticles were then dispersed via wet impregnation. The thus developed catalyst was found to be highly active and selective for water-based hydrogenation in continuous-flow. The resulting Mott-Schottky heterojunction formed between the metal and the NDC support provides high catalyst stability and enhanced activity (Scheme 64). The system could be operated for up to 40 hours without any changes and negligible metal leaching (total $11 \%$ inductively coupled plasma, ICP).

Hydrogenation of arenes and heteroarenes have also been carried out in flow. As shown earlier, selective reduction of aromatic systems is a key transformation but typically requires harsh conditions due to the aromatic stabilisation. In 2015, the Sajiki and Monguchi group developed a rhodium/rutheniumcatalysed hydrogenation in flow able to operate under relatively mild conditions (Scheme 65). A solution of the arene in iPrOH was mixed with hydrogen and the stream directed through a $30 \mathrm{~mm}$ long cartridge packed with the catalyst and heated in a range of $50-100{ }^{\circ} \mathrm{C}$. The system was shown to be efficient for several substrates, however, on some examples high pressure was still required to obtain full conversion. The authors claimed that more concentrated solutions reduced the effective contact with the catalyst thereby lowered the conversions (from $100 \%$ to $52 \%$ ) [283].

In a later publication, the Kobayashi group developed a polydimethylsilane/alumina-encapsulated $\mathrm{Rh}-\mathrm{Pt}$ bimetallic nanoparticle catalyst, $\mathrm{Rh}-\mathrm{Pt} /\left(\mathrm{DMPSi}-\mathrm{Al}_{2} \mathrm{O}_{3}\right)$ [284]. In the flow system, the two streams (gas and substrates) were introduced in the column through a double-layer structured injector. To improve the initial gas-liquid mixing, a plug of metallic mesh filter was placed before the column packed with the pregenerated catalyst. The flow apparatus was tested on 21 examples; highlighted examples are shown in Scheme 66. The hydrogenation system was left running for over 50 days $($ TON $=347,149)$ and showed no signs of deactivation. The authors claimed the activity of the catalyst was over five times higher in flow compared to batch.

The same support and encapsulation process was subsequently used to immobilise Pd nanoparticles and the flow system was found to be highly suitable for the hydrogenation of compound $222\left(\right.$ TON $=5024$, TOF $\left.=1004 \mathrm{~h}^{-1}\right)[285,286]$. This hydrogenating system proved to be useful for the multistep synthesis of rolipram (173), previously described in Scheme 38.

Due to their importance the scale up of hydrogenations have been explored by numerous industrial as well as academic
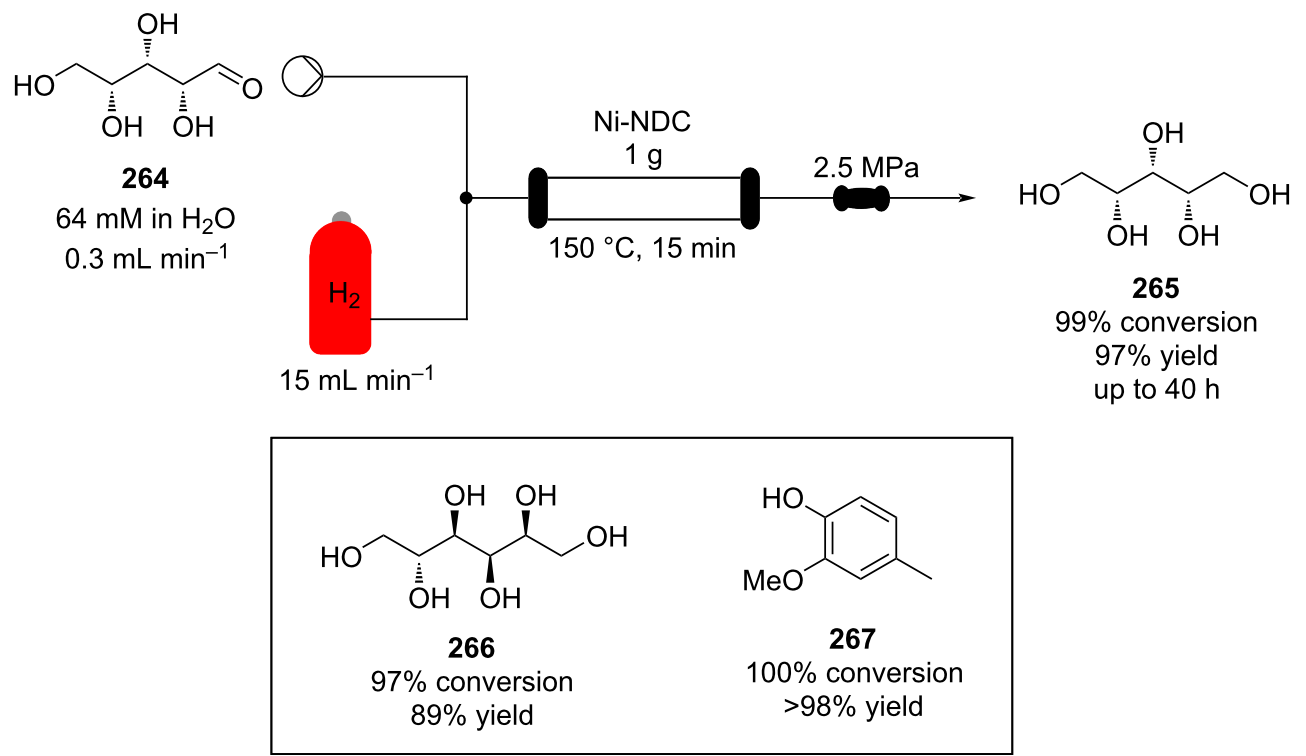


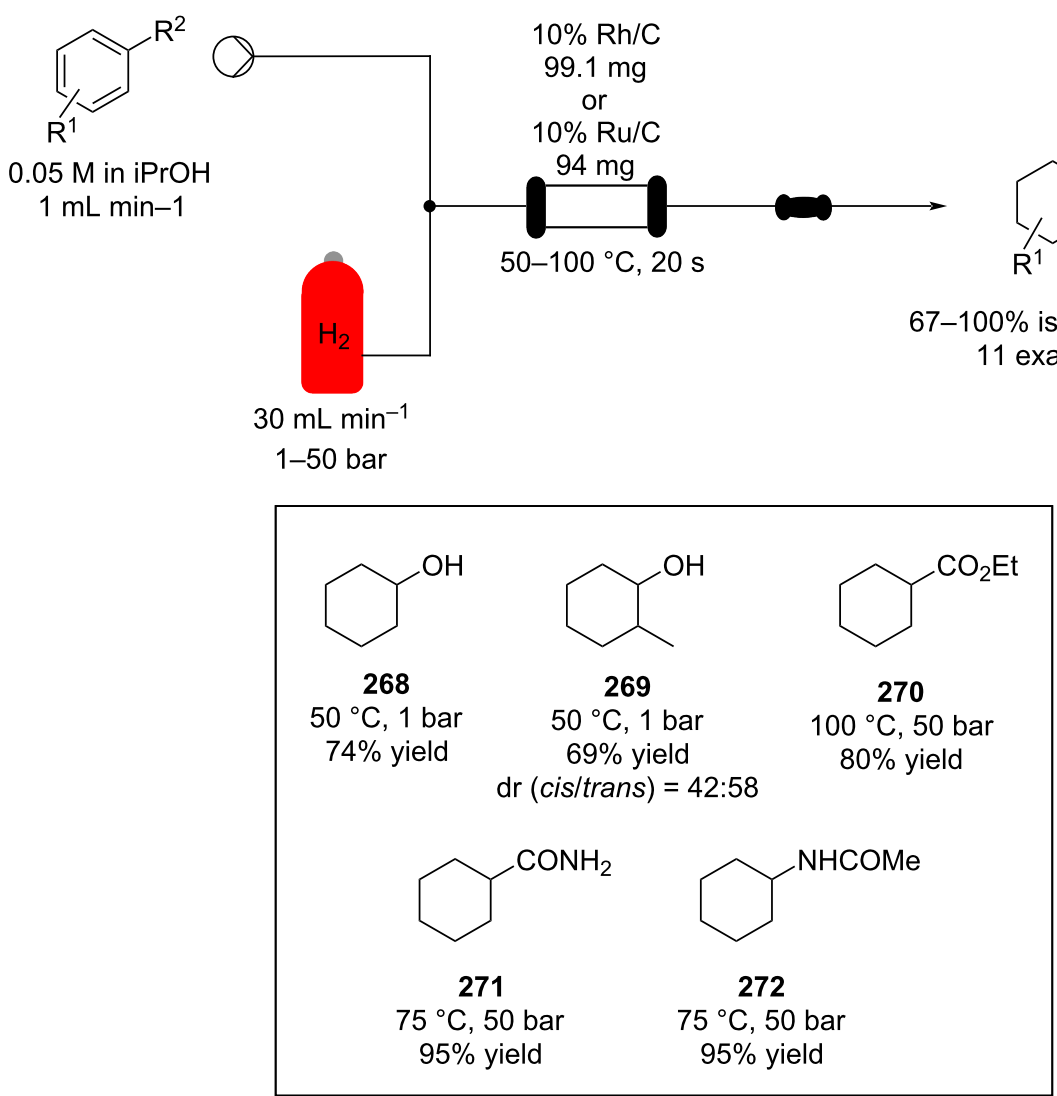

Scheme 65: Ru/C or Rh/C-catalysed hydrogenation of arene in flow as developed by Sajiki et al.

$$
\text { R }
$$

neat or $0.03-0.24 \mathrm{M}$ in $\mathrm{PrOH}$ or hexane $0.025-0.05 \mathrm{~mL} \mathrm{~min}-1$
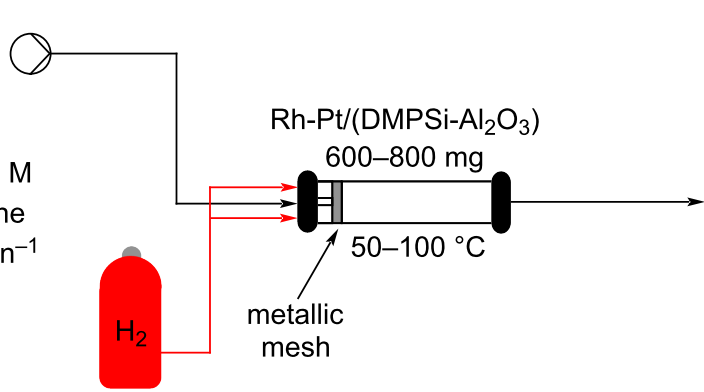

$\mathrm{Rh}-\mathrm{Pt} /\left(\mathrm{DMPSi}-\mathrm{Al}_{2} \mathrm{O}_{3}\right)$

32-63 $\mathrm{mL} \mathrm{min}-1$

$1 \mathrm{~atm}$

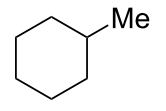

273

$>99 \%$ yield

over 50 days

TON = 347149

TOF $=1189 \mathrm{~h}^{-1}$

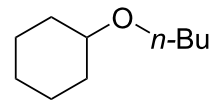

274

$>99 \%$ yield up to $23 \mathrm{~h}$

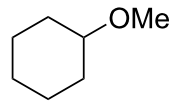

275

$>99 \%$ yield up to $30 \mathrm{~h}$<smiles>[R]C1CCCC([R])C1</smiles>

isolated yield 
organisations. A pilot scale continuous-flow synthesis of LY500307 (278), an estrogen receptor $\beta$-agonist, using an asymmetric hydrogenation was performed at Eli Lilly \& Co. (Scheme 67) [287].

A simple T-piece connector was used to introduce the gas to the liquid stream at an elevated pressure of 70 bar. During the optimisation, the starting material solubility was problematic and thus required more than $35 \mathrm{~L}$ of solvent to fully solubilise a $3.6 \mathrm{~kg}$ batch $(0.18 \mathrm{M})$. A Rh-Josiphos catalyst at a loading of $0.05 \mathrm{~mol} \%$ was found to be sufficient for the homogeneous hydrogenation. The output flow was directed into a $73 \mathrm{~L}$ plugflow tube reactor (PFR) (Figure 9) maintained at $70{ }^{\circ} \mathrm{C}$ allowing a residence time of 12 hours and enabling excellent yields with high ee $(>94 \%)$. This setup allowed a processing rate of $130 \mathrm{~L} \mathrm{~d}^{-1}$ of the starting material 276 stock solution, equating to a productivity of over $10 \mathrm{~kg} \mathrm{~d}^{-1}$ of the hydrogenation product 278.

Solubility would only rarely present an issue when considering most fragrance ingredients and their intermediates which tend to be liquid at room temperature, making them readily 'flowable', subject to viscosity. Interestingly, this also makes fragrance chemicals highly suited to operation under solvent-free conditions (SFC) or at very high concentrations in flow.

Despite the enormous range of catalysts developed for asymmetric hydrogenations, the use of chiral auxiliary methods are still commonly utilised in bulk chemical manufacture $[288,289]$. This can be attributed to their high robustness and

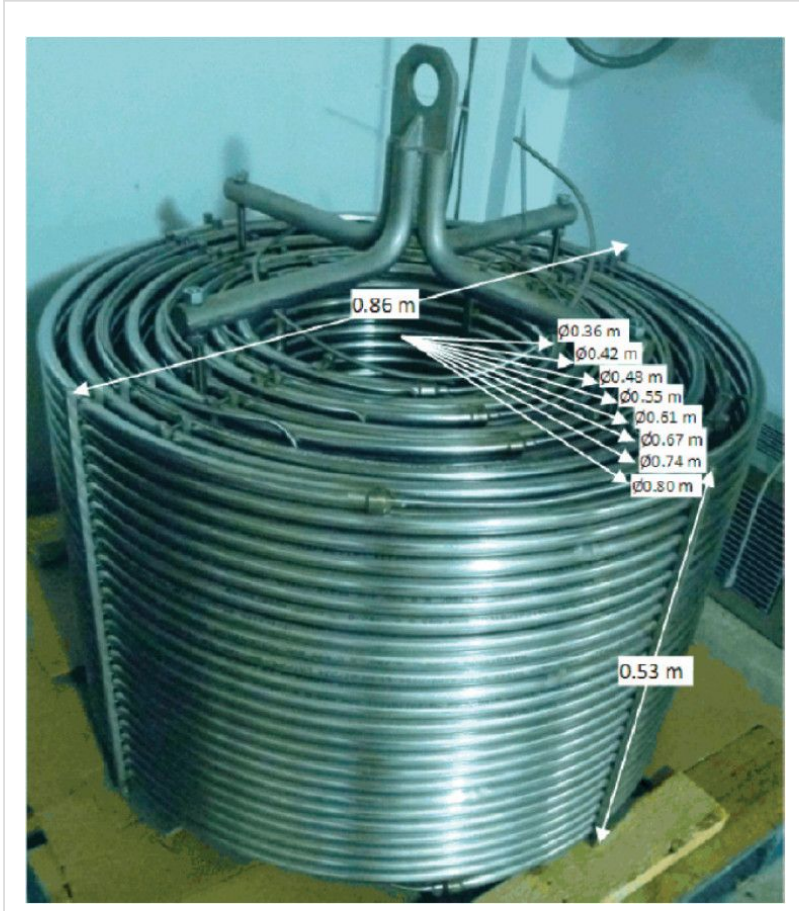

Figure 9: Picture of the PFR employed at Eli Lilly \& Co. for the continuous hydrogenation of 278 [287]. Reprinted with permission from [287] Copyright 2012 American Chemical Society.

selectivity; however, the need for additional steps and stoichiometric reagents raises concerns about their green and economic consideration. In 2018, Newman and his group addressed such concerns by developing an asymmetric hydrogenation of $\alpha, \beta$ unsaturated acids using Oppolzer's sultam 280. By telescoping

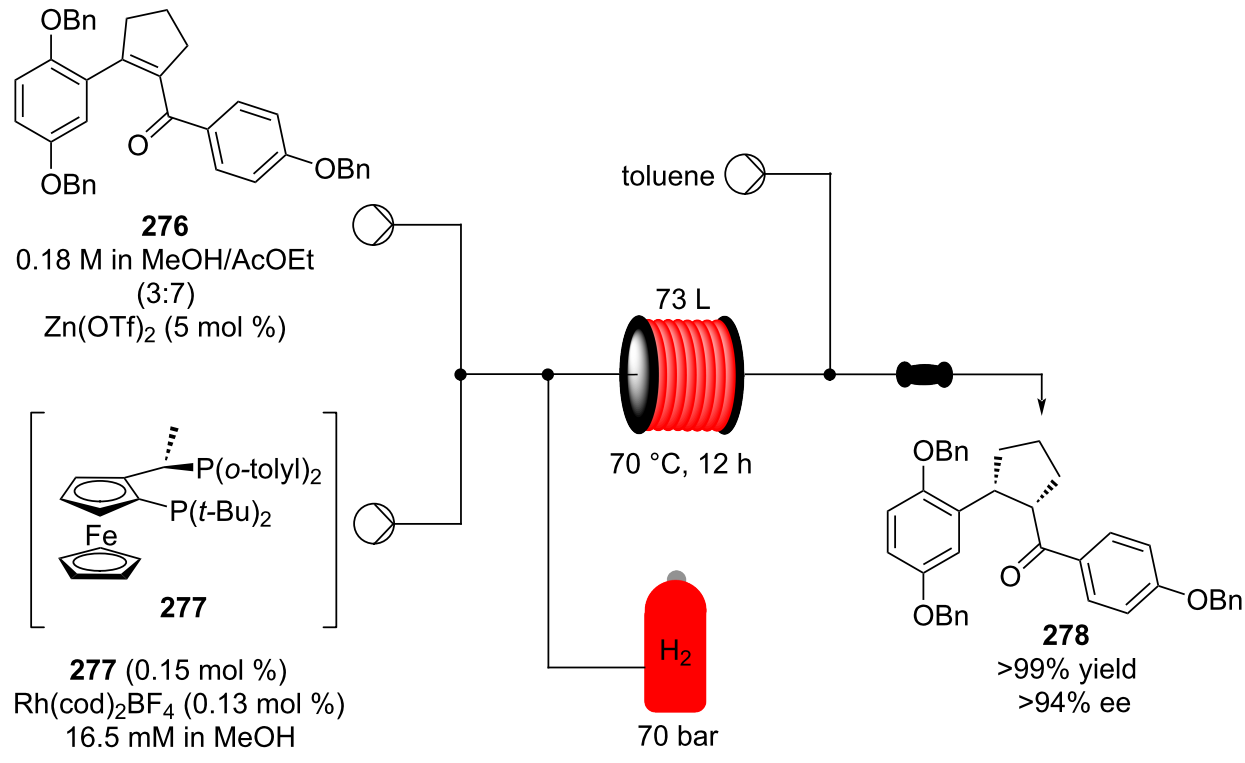

Scheme 67: High-pressure in-line mixing of $\mathrm{H}_{2}$ for the asymmetric reduction of 278 at pilot scale with a $73 \mathrm{~L}$ plug flow tube rector (PFR) coil used. 


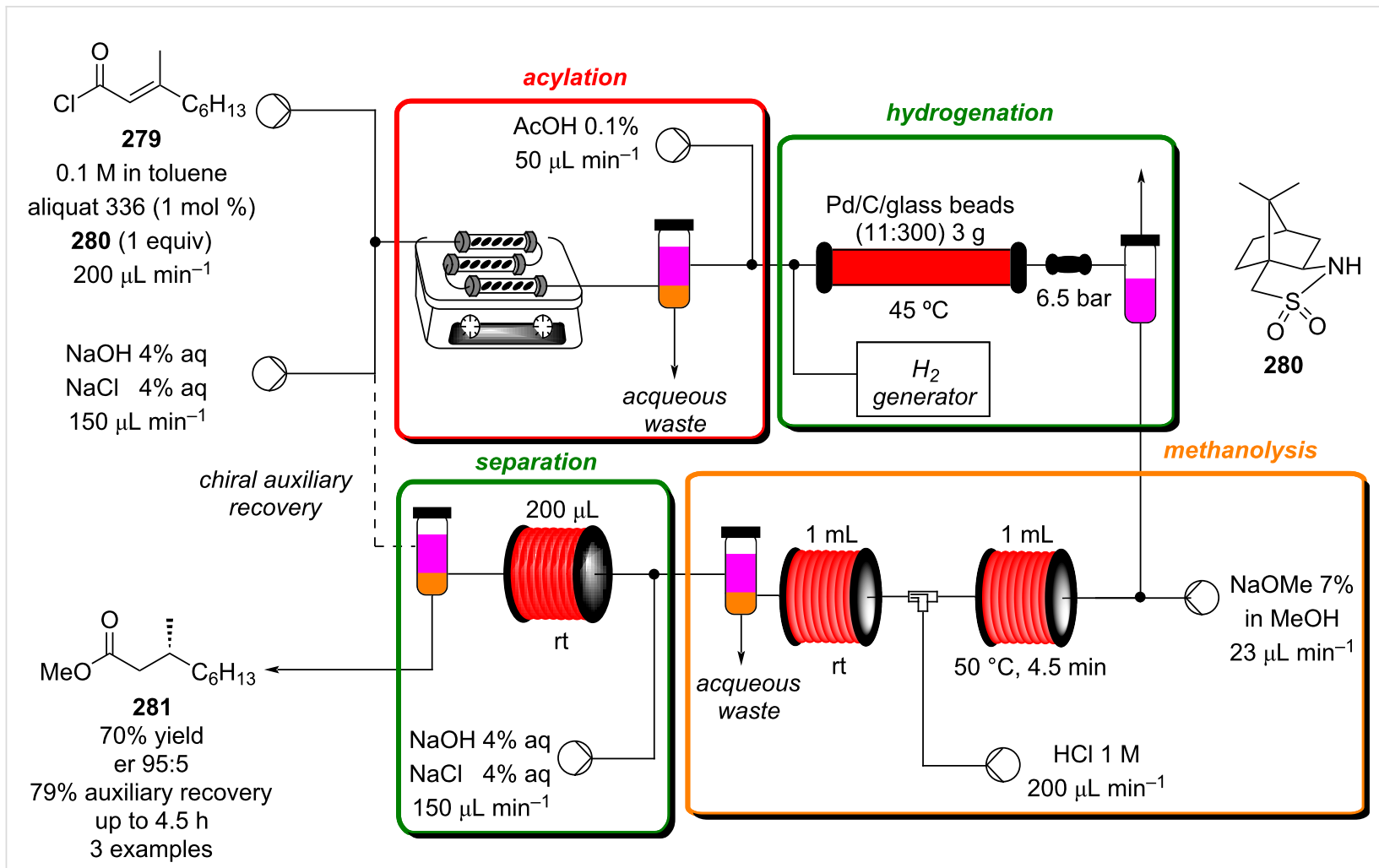

Scheme 68: Continuous-flow asymmetric hydrogenation using Oppolzer's sultam $\mathbf{2 8 0}$ as chiral auxiliary.

the three reaction steps, the preparation times reduces to 30 minutes and the auxiliary recycling efficiently improved compared to batch ( $79 \%$ recovery) [290]. The assembled flow system consists of 4 steps: acetylation, hydrogenation, methanolysis, and product/auxiliary separation (Scheme 68).

The use of PTC biphasic acylation is performed in an oscillating reactor column. The three columns were placed in series on a stirring plate and filled up with oscillating magnetic bars which created a turbulent flow increasing the phase mixing. After separation, acetic acid was mixed with the flow stream to reduce the $\mathrm{pH}$ and avoid $\mathrm{Pd} / \mathrm{C}$ catalyst deactivation by basic conditions. The hydrogenated intermediate amide was then deprotected using sodium methoxide and $\mathrm{HCl}$ as a quencher. The chiral auxiliary was recovered by selective deprotonation and continuous extraction. The apparatus allowed the recovery of Oppolzer's sultam, which can be either isolated and crystallised or cycled back in the flow system where it could be reused eight times (4.5 hours of runtime).

\section{Oxidation and related transformations}

The formation of aldehydes/ketones from alcohols or other functionalities is powerful when considering the dissonant olfactory characteristics of products comprised of such moieties (Scheme 69).
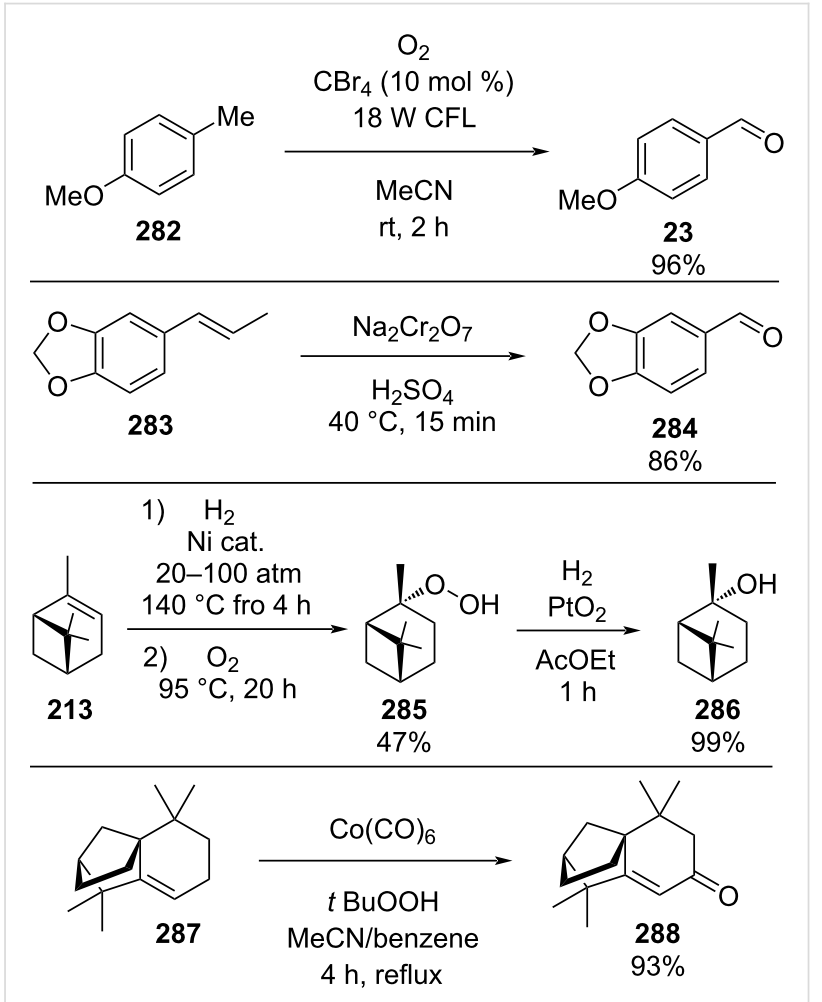

Scheme 69: Some examples of industrially important oxidation reactions in the F\&F industry. CFL: compact fluorescence light. 
Examples of such oxidation reactions include the synthesis of $p$-anisaldehyde (23) from anisole (282) [291], the formation of piperonal (284) from isosafrole (283) [292,293] and the generation of pinane hydroperoxide (285) from cis-pinane (the hydrogenation product of $\alpha$-pinene (213)) [294]. Additional procedures such as the oxidation of isolongifolene (287) to isolongifolenone (288) using tert-butyl hydroperoxide and cobalt hexacarbonyl [295] are also important industrial sequences.

The disposal of metal waste streams such as those containing manganese and chromium oxides are heavily regulated and so recycling/recovery is necessary. Current industrial focus is now being increasingly placed on finding greener oxidation alternatives, such as the use of elemental oxygen. Indeed, many oxidation protocols rely on the use of gaseous $\mathrm{O}_{2}$, so economic and safety implications addressed in the previous section are also applicable here. Like with $\mathrm{H}_{2}$, in-line mixing of $\mathrm{O}_{2}$ is possible and it has also been successfully incorporated into the tube-intube reactor setups.

Aerobic oxidation in flow under both hetero- and homogeneous catalysis conditions has been extensively reviewed with special emphasis on the improvement in safety [296,297]. In 2009, the use of a gold wall-coated capillary reactor for the oxidation of alcohols with molecular $\mathrm{O}_{2}$ was reported (Scheme 70) [298].

The column was prepared by attaching microencapsulated gold onto amine functionalities within a modified polysiloxane capillary performed at elevated temperatures. The optimisation of the oxidation process was carried out on 1-phenylethanol (250) and the established conditions were then applied to 9 additional substrates. Good yields were obtained in all cases except for the oxidation of benzyl alcohol which required a column coated with a bimetallic Pd/Au matrix. Despite the low flow rates necessary and subsequent low throughput, the column was found to be highly robust with no Au leaching even after four days of continuous processing.

Heterogeneous catalyst systems with improved throughput have subsequently been developed. Uozumi and co-workers reported a continuous process in which a column reactor containing platinum nanoparticles dispersed on an amphiphilic polystyrene-poly(ethylene glycol) resin (ARP-Pt) was used to effect oxidation of alcohols (Scheme 71) [299]. By using an $\mathrm{X}-\mathrm{Cube}^{\mathrm{TM}}$ reactor, much shorter residence times and higher throughputs were possible employing this catalytic system. In-line mixing of the $\mathrm{O}_{2}$ (50 bar) was used with column contact times of less than one minute, effecting full conversion of various alcohols evaluated. The reactor was additionally used for a range of 28 aldehydes, all of which gave the corresponding carboxylic acids in good yields. Other examples of heterogeneous aerobic oxidation of alcohols in flow include the use of a $\mathrm{Ru}(\mathrm{OH})_{\mathrm{x}} / \mathrm{Al}_{2} \mathrm{O}_{3}$ catalyst packed bed reactor [300,301] and carbon black-stabilized polymer-incarcerated gold/platinum bimetallic nanocluster catalysts (PI-CB/Au-Pt) [302]. In-line oxygen delivery has also been performed using the tube-in-tube reactor system whilst making use of a downstream packed bed $\mathrm{Au}-\mathrm{Pd} / \mathrm{TiO}_{2}$ PTFE reactor [303]. Metal organic framework (MOF) have also been considered as potential candidates for heterogeneous catalysts due to their high surface area and porosity. Flow systems exploring this area have been developed and recently reviewed by Garcia and Dhakshinamoorthy [304].

There has also been significant effort directed towards systems enabling homogeneous aerobic flow oxidation. The work conducted by Favre-Réguillon et al. has revealed that the autoxidation of aldehydes in flow using elemental $\mathrm{O}_{2}$ is possible with

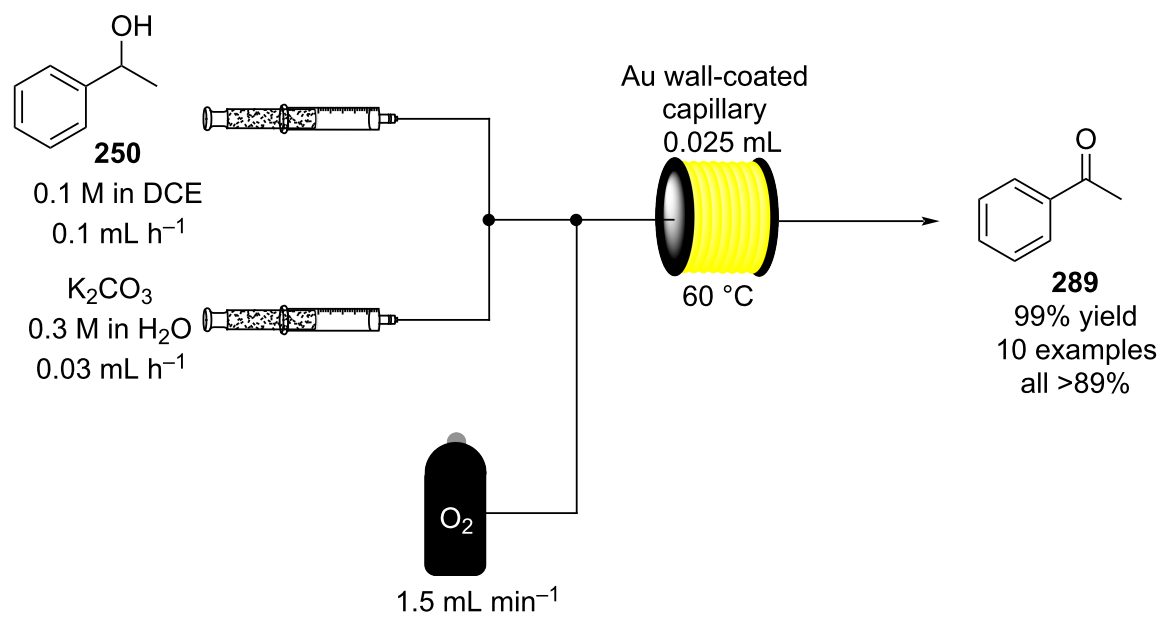

Scheme 70: Gold-catalysed heterogeneous oxidation of alcohols in flow. 

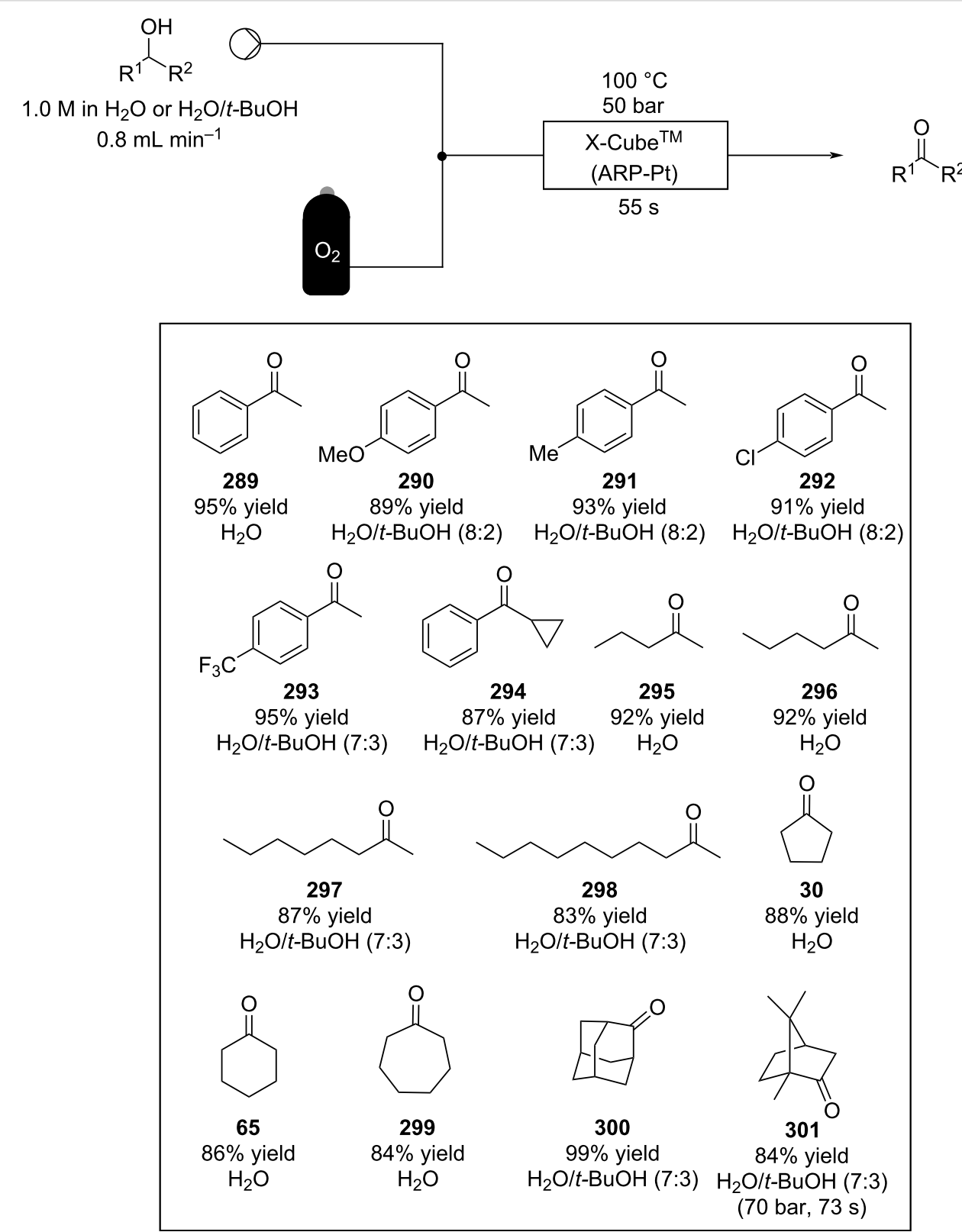

Scheme 71: Uozumi's ARP-Pt flow oxidation protocol.

and without the use of a catalyst (Scheme 72) [305,306]. In-line $\mathrm{O}_{2}$ mixing at 5 bar was used and the incorporation of in-line GC-MS analysis allowed for high-throughput screening, facilitating rapid optimisation of the reaction conditions. The addition of manganese and chromium salts enhanced conversions and selectivity towards the carboxylic acid such that generation of the problematic Criegee intermediate was almost completely avoided.

In 2020, Kappe and co-workers employed the aerobic oxidation of aldehydes to acids to develop a de novo synthesis of $\gamma$-nitrobutyric acids, key intermediates of several GABA deriva- tives. The authors described a highly effiencient and selective two-step telescoped synthesis with productivities ranging between 2.77 and $3.14 \mathrm{~g} \mathrm{~h}^{-1}$ [307].

A method for performing permanganate-mediated oxidation of alcohols and aldehydes to carboxylic acids as well as the Nef oxidations of nitroalkanes was developed in 2010 [308], in this process, ultrasound was employed to aid with the continuous processing of downstream $\mathrm{MnO}_{2}$ slurries which prevented fouling of the reactor (Scheme 73). This was one of the early examples of overcoming the potential clogging issue when solids are produced in flow as part of the product stream. Soni- 

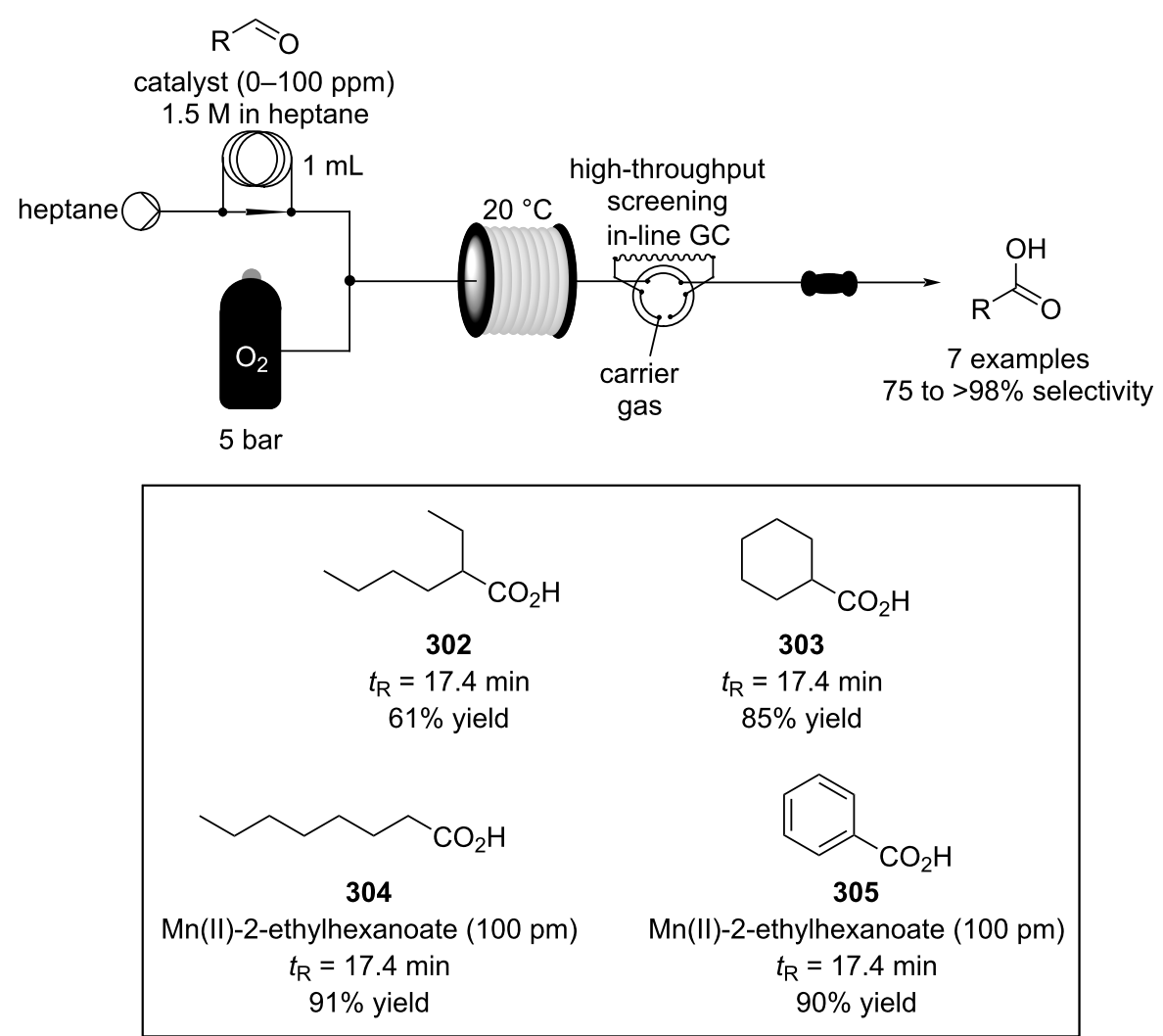

Scheme 72: High-throughput screening of aldehyde oxidation in flow using an in-line GC
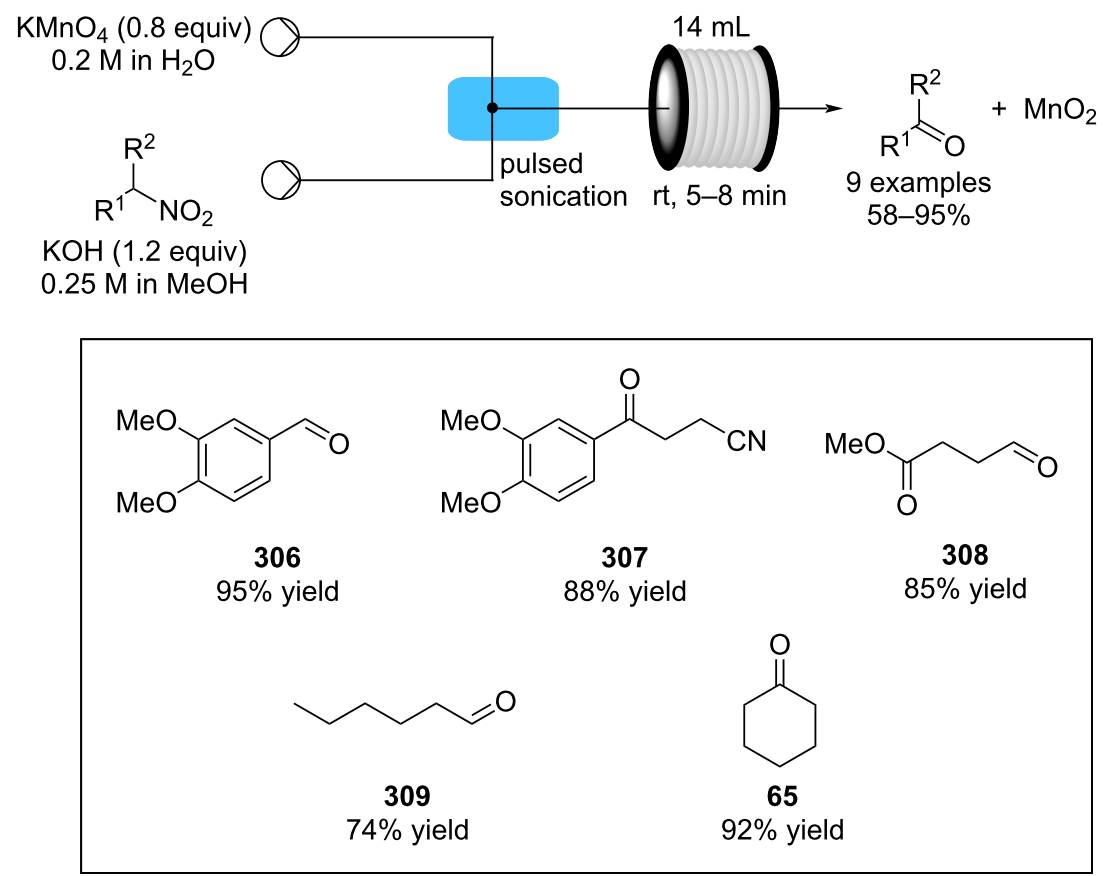

Scheme 73: Permanganate-mediated Nef oxidation of nitroalkanes in flow with the use of in-line sonication to prevent reactor clogging. 
cation or mechanical agitation have subsequently become a common tool to prevent accumulation and blockage in a flow channel [44-48].

In 2013 a continuous-flow aerobic anti-Markovnikov Wacker oxidation was reported by Ley and Bourne (Scheme 74) [309]. The precise control of reaction conditions afforded by the microreactor system and the tube-in-tube reactor technology made it possible to use gaseous $\mathrm{O}_{2}$ for the oxidation of olefins to aldehydes while avoiding overoxidation. Selectivity was finetuned by systematic modification of the reaction parameter (oxygen pressure, $\mathrm{H}_{2} \mathrm{O}$ equivalents and reactor temperature), resulting in a system that was used for the selective oxidation of 12 different functionalised styrene derivatives (77-92\% selectivity). A larger scale production of 2-(4-chlorophenyl)acetaldehyde (302), utilising a "double dosing" of $\mathrm{O}_{2}$ (Scheme 74), boosted the throughput seven-fold to $21.6 \mathrm{mmol} \mathrm{h}^{-1}$, yielding $17.6 \mathrm{~g}$ of the aldehyde $\mathbf{3 1 1}$ after 6 hours of processing.
In 2019, Gupton et al. explored the use of an X-Cube ${ }^{\mathrm{TM}}$ reactor for the aerobic oxidation of benzaldehyde (17) using a commercial palladium catalyst. When the tube-in-tube apparatus was placed in-line, the conversions increase 9-fold using oxygen (31\%) and 5-fold using air (10\%) as the oxidant. The authors claimed high selectivity $(100 \%)$ and high productivity (STY = $22000 \mathrm{~g} \mathrm{~L}^{-1} \mathrm{~h}^{-1}$ using air, $64000 \mathrm{~g} \mathrm{~L}^{-1} \mathrm{~h}^{-1}$ using oxygen) at lower pressure (2.8 bar) compared to the results acquired using higher pressures (25 bar) [310].

Other examples of homogeneous gas-liquid aerobic oxidation in flow include the direct oxidation of 2-benzylpyridines (Scheme 75). The system used employed air as the oxidant and propylene carbonate (PC) as a green solvent. The authors pointed out the usage of PC and the flow system allowed them to work at high temperature reducing the reaction time to 13 minutes, compared to 1 hour in batch mode [311].

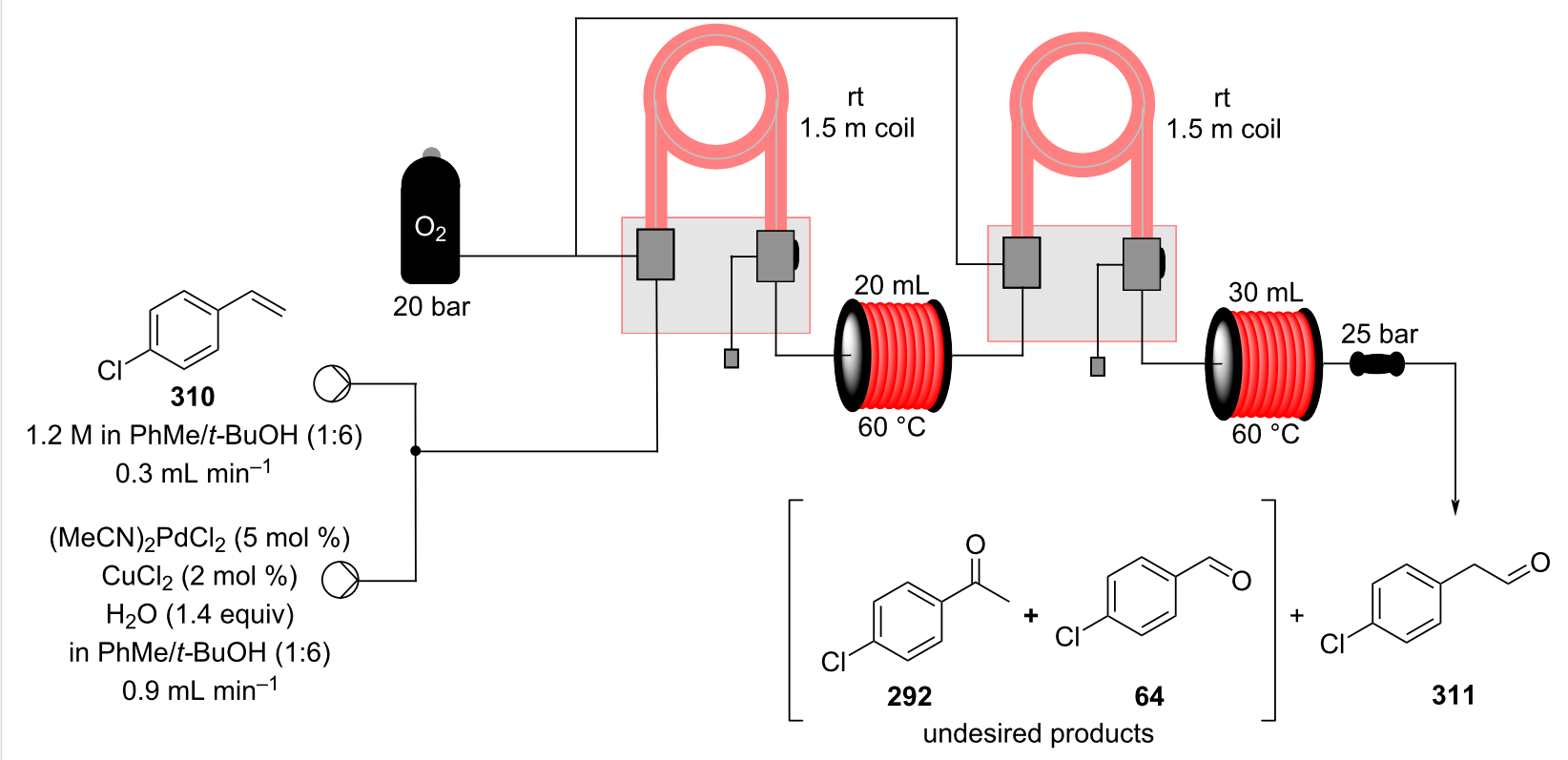

Scheme 74: Continuous-flow aerobic anti-Markovnikov Wacker oxidation.<smiles>c1ccc(Cc2ccccn2)cc1</smiles>

312

1.2 $\mathrm{M}$ in $\mathrm{PC}$

$\mathrm{FeCl}_{3}(5 \mathrm{~mol} \%)$

$0.6 \mathrm{~mL} \mathrm{~min}^{-1}$

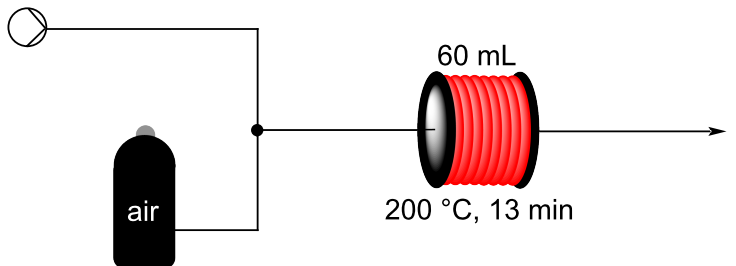

$1.7 \mathrm{~L} \mathrm{~min}^{-1}$

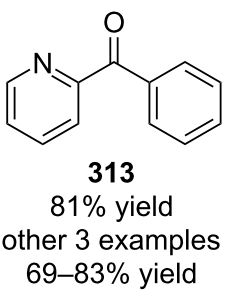

$69-83 \%$ yield 
Of particular interest to many chemists in the industry is photooxidation in flow. Indeed, a number of such transformations have already been investigated on fragrance derivatives. As with heat transfer, the high apparent surface contact area in flow makes it highly compatible with procedures in which light/radiation transmittance is key. The continuous-flow photo-oxygenation of hexamethylbenzene [312] and monoterpenes [313] has been demonstrated by Park et al. Several additional substrates such as $\beta$-pinene (289), $\alpha$-pinene (188), $\delta$-limonene (9), $(-)$-citronellol (299) and $\alpha$-terpinene have all been explored in various oxidative transformations and comparisons to batch protocols have been made (Scheme 76). As is typical of photooxygenation processes, batch experiments required longer reaction times and generally gave poor selectivities. The reaction of $\beta$-pinene (314), for example, gave the four products 316-319 upon treatment with oxygen and a sensitizer (desired product,
$51 \%$ ) after 24 hours in batch. However, the desired product (316) could be obtained exclusively in $99.9 \%$ yield after just 30 minutes in flow. Similar observations have been made for the formation of the cyclohexenol 320, from $\delta$-limonene (9), with a marked improvement upon reaction time, selectivity and yield observed in flow.

A polydimethylsiloxane-based single channel microreactor has been engineered with direct in-line mixing of $\mathrm{O}_{2}$ via a T-junction or via presaturation of the reagent input solution [314]. After optimisation of the reaction conditions and profiling of the reactor, a tube-in-tube reactor approach was used for scaleup procedures, affording significant improvements over batch methods in terms of daily product output. Irradiation using a lamp within a vacuum bath (inner walls mirror coated) proved very effective in this instance. Additional work by Seeberger et

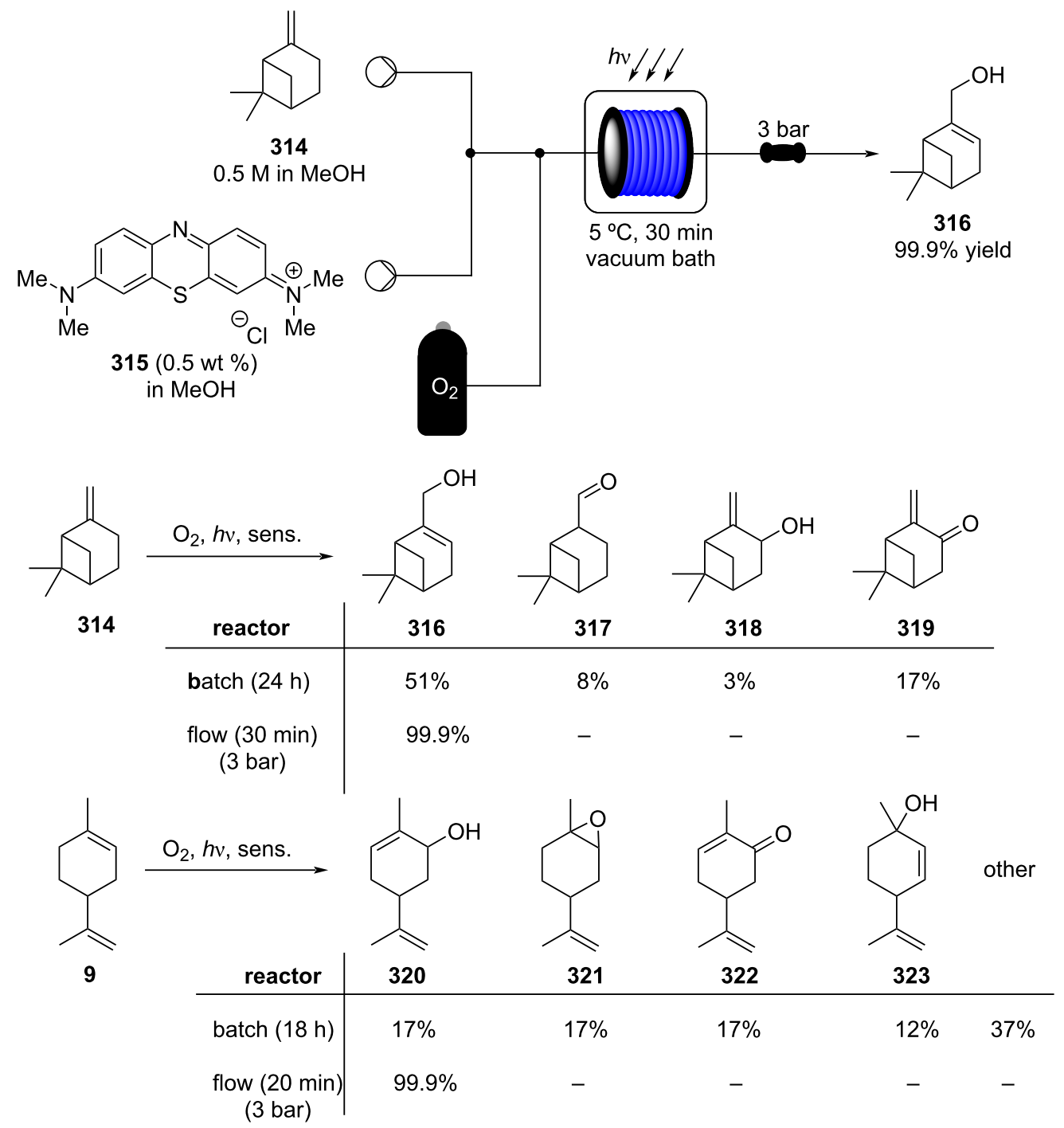

Scheme 76: Continuous-flow photo-oxygenation of monoterpenes. 
al. [315] has demonstrated an approach based upon the original GSK-Booker-Milburn-type reactor [316], where the reactor coil surrounds the light source generating singlet oxygen in situ (Scheme 77). This design was successfully applied to the oxidation of substrates such as citronellol (299), $\alpha$-pinene (188) and 2-methyl-2-butene.

Enzymes have also been productively incorporated into flow systems, leading to novel technologies such as "packed-bed microbioreactors" [114,317,318]. Enzyme-catalysed gas-liquid reactions have been exploited in falling-film microreactor (FFMR) for example for the glucose oxidase (GOx)-mediated oxidation of $\beta$-D-glucose to gluconic acid (Scheme 78) [319]. The FFMR was designed to create a thin film interface of the substrate solution and gas (thickness in the range of $10-100 \mu \mathrm{m})$ leading to a high gas-liquid contact area. The high surface area to volume ratio also conveniently allows for efficient heat transfer in exothermic processes.<smiles>CC(C)=CCCC(C)CCO</smiles>

324

$0.5 \mathrm{M}$ in $\mathrm{MeOH}$ $\operatorname{TPP}(0.4 \mathrm{~mol} \%)$

$5 \mathrm{~mL} \mathrm{~min}{ }^{-1}$

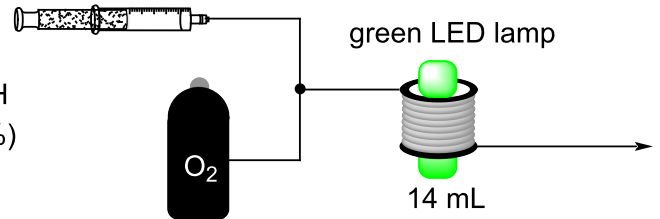

$12 \mathrm{~mL} \mathrm{~min}{ }^{-1}$
$0.8 \min , 25^{\circ} \mathrm{C}$<smiles>CC(C/C=C/C(C)(C)O)CCO</smiles><smiles>C=C(C)[C@H](O)CCC(C)CCO</smiles>

326

325/326 1.1:1

$88 \%$ conversion

Scheme 77: A tubular reactor design for flow photo-oxygenation.

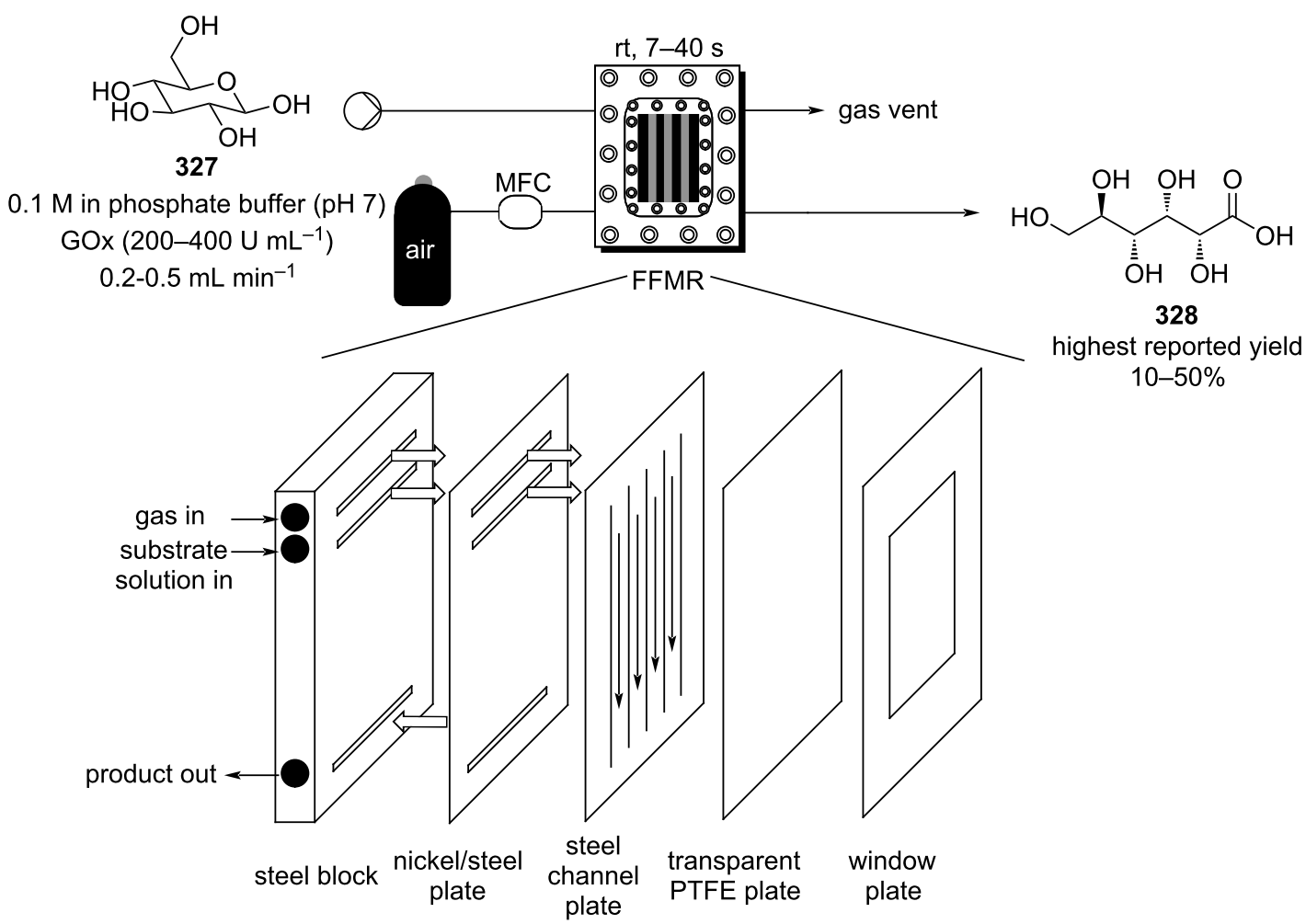

Scheme 78: Glucose oxidase (GOx)-mediated continuous oxidation of glucose using compressed air and the FFMR reactor. 
More recently, a continuous-flow aerobic oxidation of primary alcohols with a $\mathrm{Cu}(\mathrm{I}) / \mathrm{TEMPO}$ catalyst system was achieved (Table 7) [320]. This was developed as an alternative to a previously reported palladium-catalysed continuous-flow protocol [321], with the new $\mathrm{Cu}(\mathrm{I}) / \mathrm{TEMPO}$ methodology offering shorter residence times and decreased susceptibility to catalyst decomposition as well as being applicable to a broader substrate scope.

Of the 9 substrates tested (Table 7), all gave yields of $95 \%$ and above and a $100 \mathrm{~g}$-scale version based upon benzyl alcohol (16) oxidation was carried out over a 24 hour period with a sustained yield of $>99 \%$. To improve the safety of the process a $9 \% \mathrm{O}_{2}$ in $\mathrm{N}_{2}$ mixture was used as the oxygen source thus avoiding the highly flammable oxygen and explosive composition.
TEMPO has also been utilised as a transfer catalyst for selective alcohol oxidation using in situ-generated sodium hypochlorite as a stoichiometric oxidising agent [322]. Chlorine was produced by adding hydrochloric acid to solid $\mathrm{MnO}_{2}$ and the gas stream directed to react with a flow of aqueous sodium hydroxide to yield sodium hypochlorite. To prevent its rapid decomposition, a buffer solution was combined to adjust the $\mathrm{pH}$ to around 9.5 (Scheme 79). The individual flow rates were then set in order to generate a specified range of $\mathrm{NaOCl}$ concentration, which had the correct strength to avoid the production of overoxidation products. In this setup, the alcohols along with TEMPO were mixed with the oxidising agent $(\mathrm{NaOCl})$ at $0{ }^{\circ} \mathrm{C}$. A mixture of geranial $((Z)-79)$ and neral $((E)-79)$ in a 6:4 ratio was produced in $80 \%$ yield, additionally 7 other aldehydes were synthesized in moderate to good yields.

Table 7: Continuous-flow aerobic oxidation of primary alcohols with $\mathrm{Cu}(\mathrm{I}) / \mathrm{TEMPO}$.

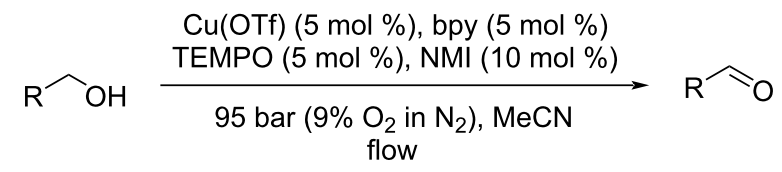

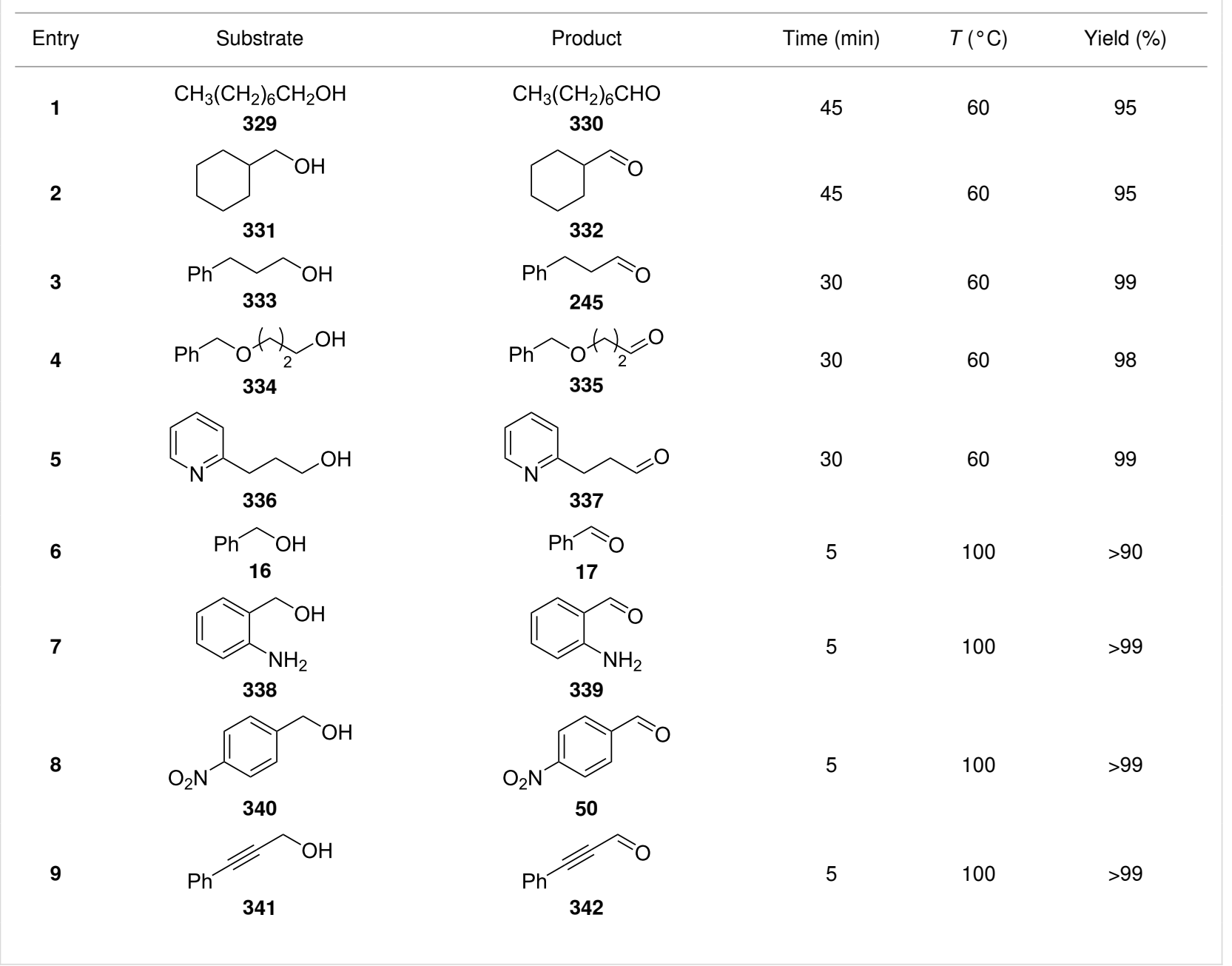




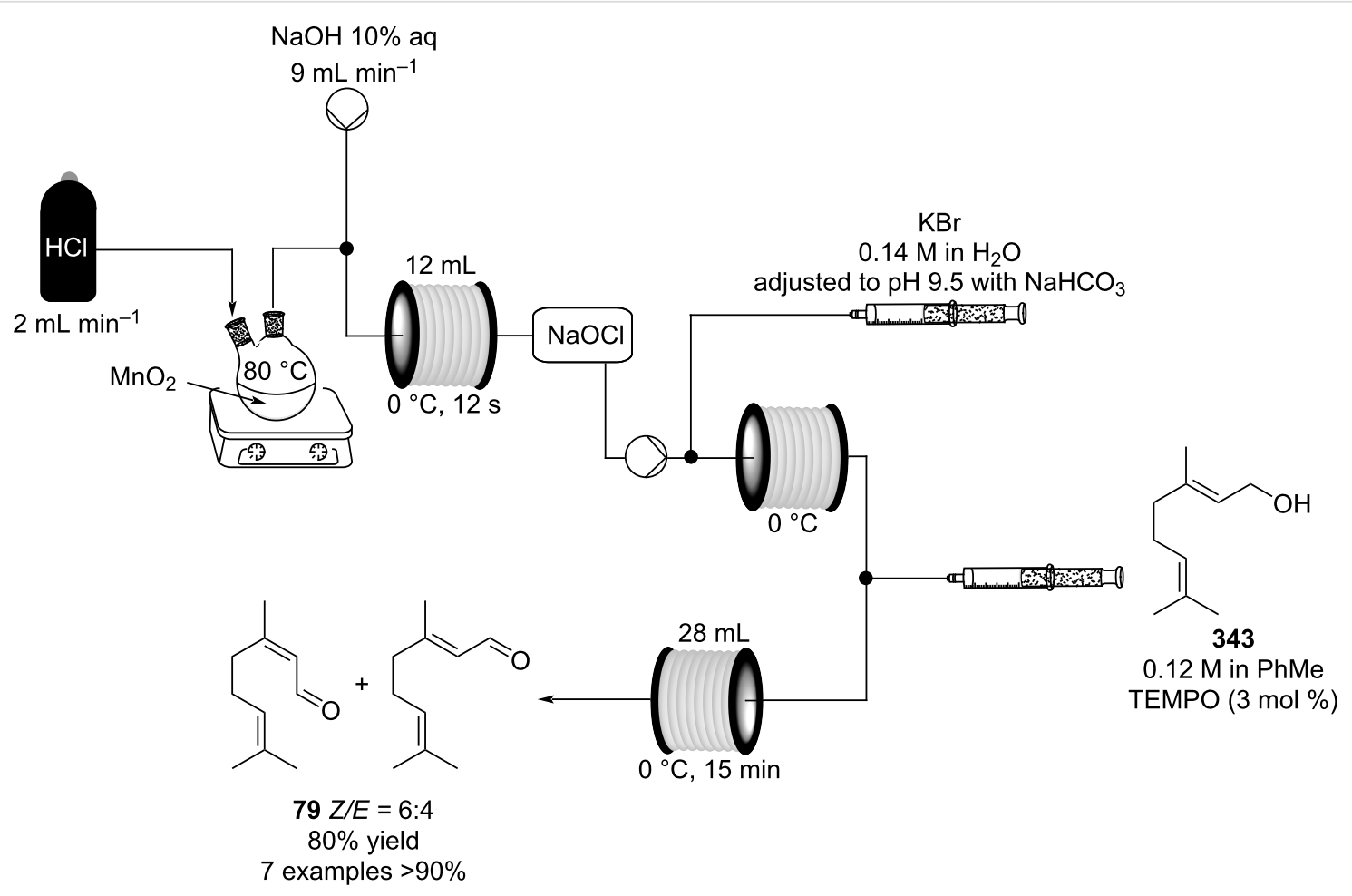

Scheme 79: Schematic continuous-flow sodium hypochlorite/TEMPO oxidation of alcohols.

Immobilisation of TEMPO ((2,2,6,6-tetramethylpiperidin-1yl)oxyl) has also been explored. In 2009, TEMPO was efficiently tethered on an AMBERZYME ${ }^{\circledR}$ oxirane resin [323] The catalyst was then introduced into a fluoroelastomeric tube and wrapped around metal bars used as a heat sink cooling system. The reaction media was formed of two phases: organic, and aqueous phase. The segmented flow produced provides enough phase interaction to allow a rapid oxidation of benzyl alcohol (16, 4.8 minutes). The use of the system resulted in efficient oxidation of many different types of aromatic and aliphatic alcohols, some highlighted examples are depicted in Scheme 80. Beneficially, the heterogeneous catalyst remains highly active even after hours of operation (up to 9 hours). Other TEMPO-immobilised systems have also been developed Sato and Okuno et al. explored immobilisation on a polyethylene-polyacrylic acid polymer, and Smarsly et al. using mesoporous silica particles [324,325]. Each support strategy offers different processing advantages and limitations based upon pressure drop, loading and ease of preparation. Herman and his group developed a continuous triphasic aerobic oxidation which involved nitric acid as co-oxidant and employed a silica-immobilised TEMPO [326].

Phase transfer catalysts (PTC) in biphasic oxidation reaction are well-known and described widely in the literature [327]. In fact, PTC-assisted oxidations have been comprehensively reviewed by Penso et al. in 2016. Flow examples have also been successfully conducted. As an example, the use of bleach and catalytic tetrabutylammonium bromide (TBAB) for the oxidation of alcohols and aldehydes in flow was reported by Jamison et al. in 2012 (Scheme 81) [328]. The simple setup and relatively safe reagent combination makes the approach particularly attractive [329].

Continuous-flow biphasic PTC-assisted oxidations using $\mathrm{NaOCl}$ has also been explored by Jensen and his group using a Corning Low Flow reactor (LFRs) and advanced flow reactors (AFRs). These types of mesoscale device allowed easy scale-up of a process up to $4 \mathrm{~g} \mathrm{~min}^{-1}$ without losing efficiency [330]. The same group later developed a flow system using hydrogen peroxide which was able to quantitatively transform $\mathbf{2 5 0}$ into acetophenone (289, Scheme 82 ). The authors pointed out the simplicity of scaling up the process from an $160 \mu \mathrm{L}$ microreactor to an $100 \mathrm{~mL}$ reactor (AFR) retaining the same processing efficiency [331]. To improve the phase separation, the authors employed a set of in-line PTFE membranes which enabled over $90 \%$ of the PTC to be extracted from the product.

Epoxidation in flow represents a potentially valuable tool for fragrance and flavour chemists with perceivable safety implications for the handling of hazardous or unstable epoxidising agents and reactive intermediates. 


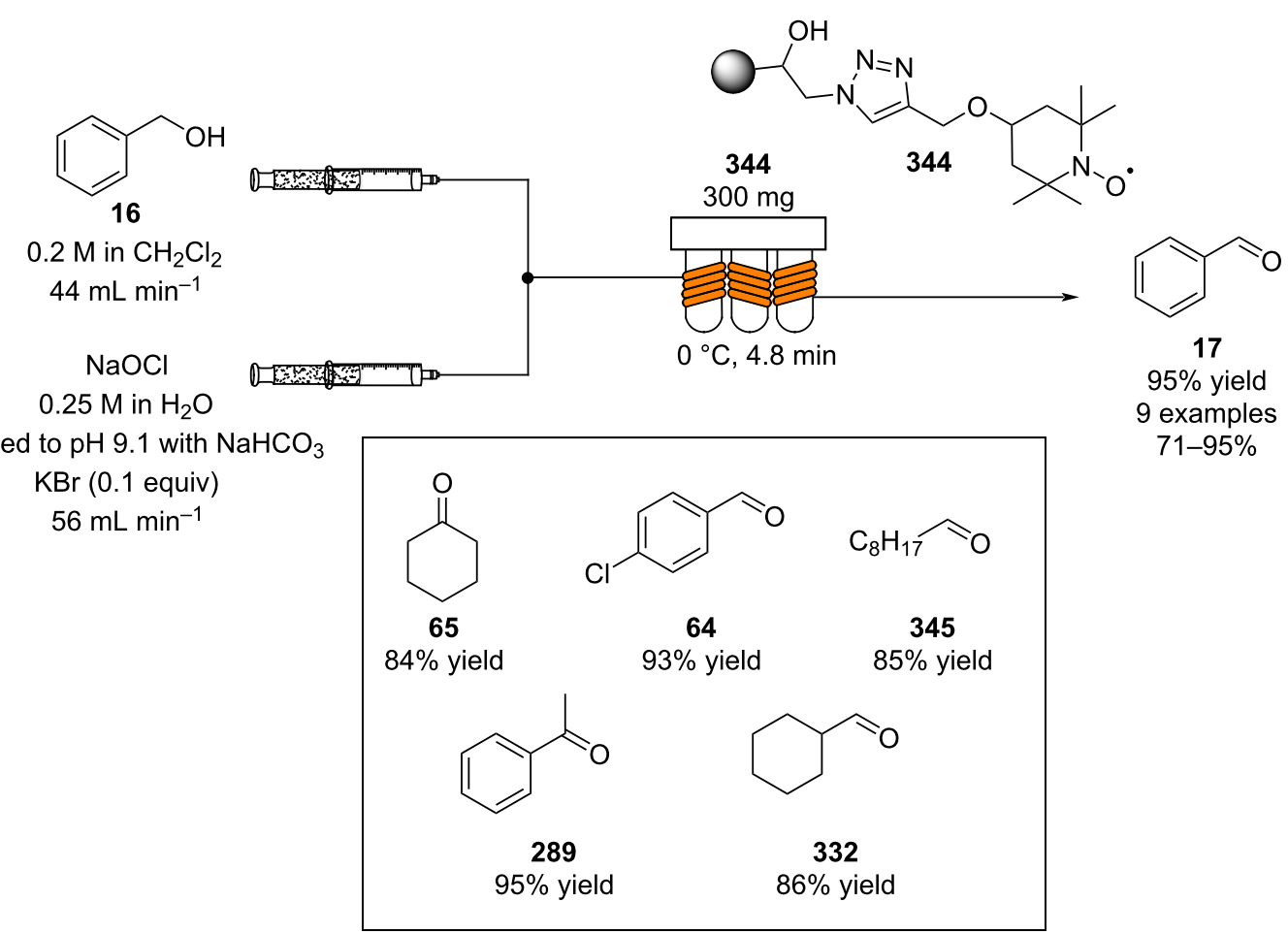

Scheme 80: Oxidation using immobilised TEMPO (344) was developed by McQuade et al.

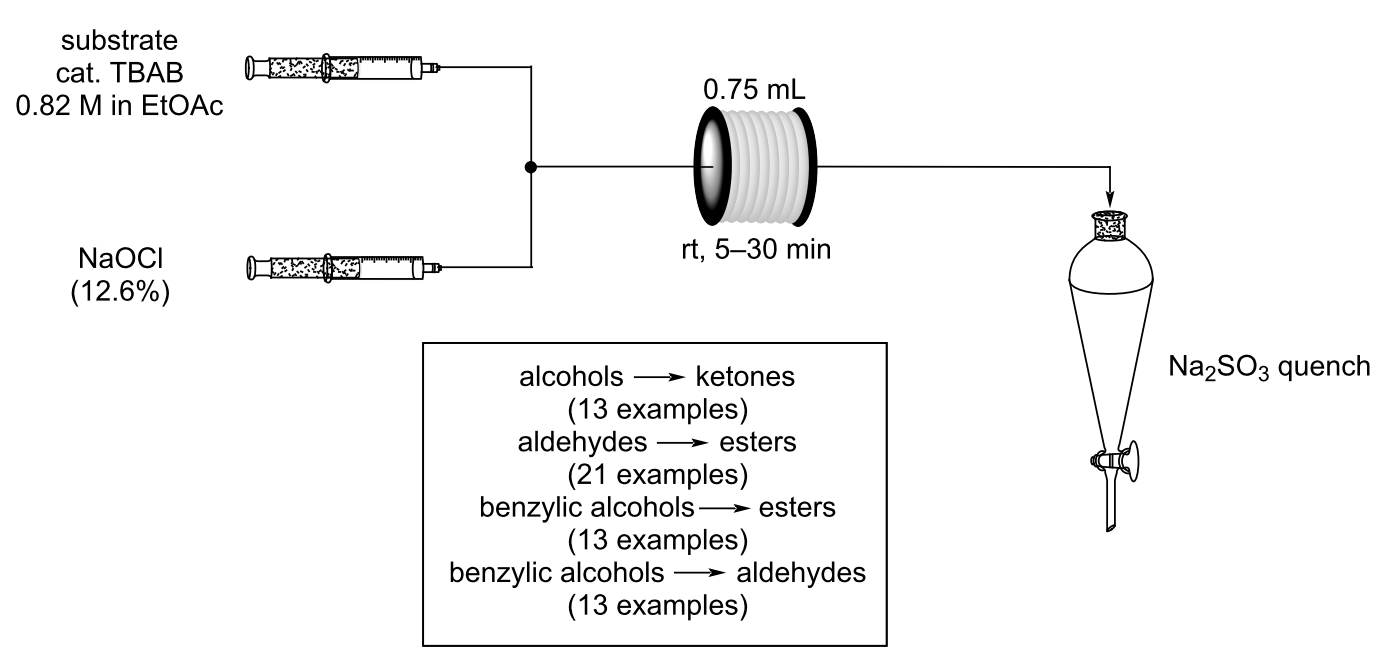

Scheme 81: General protocol for the bleach/catalytic TBAB oxidation of aldehydes and alcohols.

In 2016, Guo et al. addressed the problem of handling the hazardous epoxidising agent $m$-chloroperbenzoic acid ( $m$-CPBA) by in situ preparation from the corresponding acyl chloride and potassium peroxide (Scheme 83) [332]. The group explored an engineered micromixer to increase mass transfer for the biphasic system. Further optimisation of the process allowed the yield of the epoxidising agent to be enhanced to $87 \%$, and obtaining better selectivity than material provided by commer- cial suppliers. The setup was ultimately utilized for cyclohexene oxide preparation. Of particular value was that upon exiting the capillary tube reactor, the biphasic solution undergoes a continuous phase separation through an engineered device, which is based on the principles of inclined plate settler. The optimised flow apparatus allowed collection of $97 \%$ of the organic phase and isolation of cyclohexene oxide (349) in 100\% conversion and $95 \%$ selectivity. The authors highlighted the 


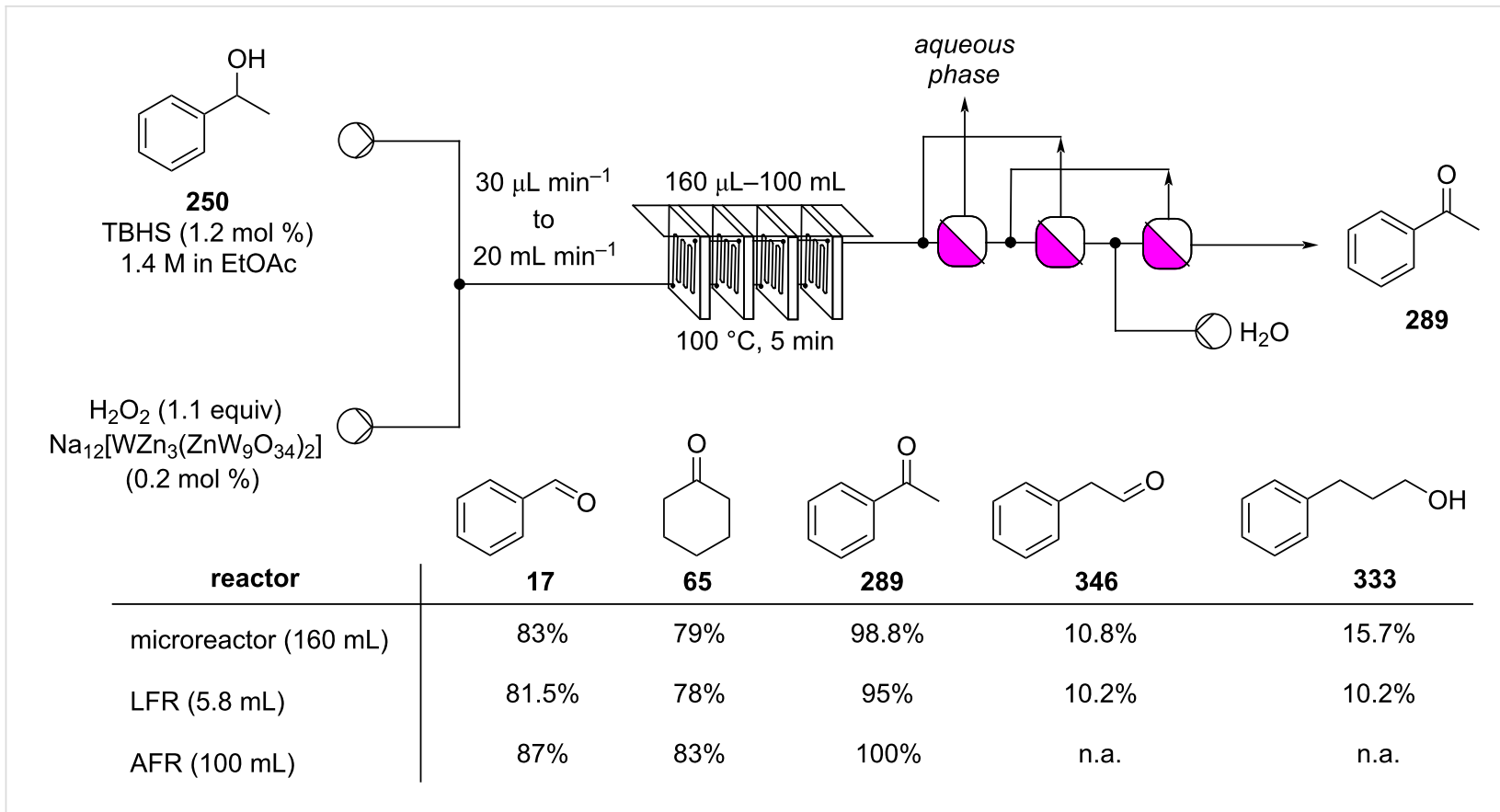

Scheme 82: Continuous-flow PTC-assisted oxidation using hydrogen peroxide. The process was easily scaled up by different types of microreactors (LFR and AFR).

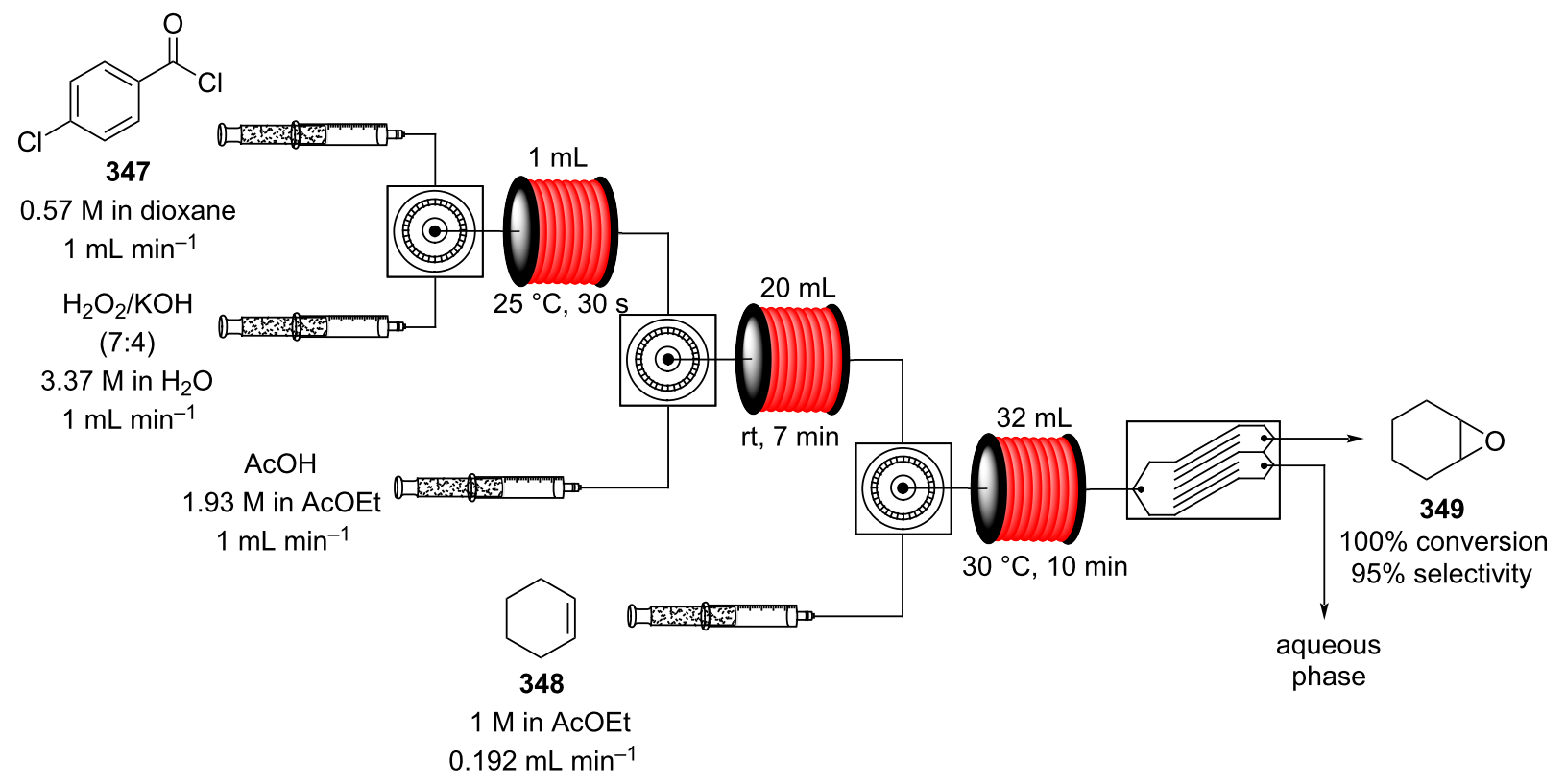

Scheme 83: Continuous-flow epoxidation of cyclohexene (348) and in situ preparation of $m$-CPBA.

advantage of the in situ preparation of $m$-CPBA, which cuts down the cost of a possible industrial production. The same group developed a similar system for the preparation of epoxidised soybean oil [333].

In situ formation of dimethyldioxirane (DMDO) has also been employed for the epoxidation of miscellaneous alkenes by
McCluskey and his group in a flow based process [334] (Scheme 84). Acetone was used as the solvent in the alkene solution, and solutions of $\mathrm{NaHCO}_{3}$ and Oxone ${ }^{\circledR}$ were directly mixed with the main stream. The author showed how important the temperature was, being maintained at $60{ }^{\circ} \mathrm{C}$, no epoxide formation was detected below this critical temperature and a too high temperature generated multiple byproducts. The generated 


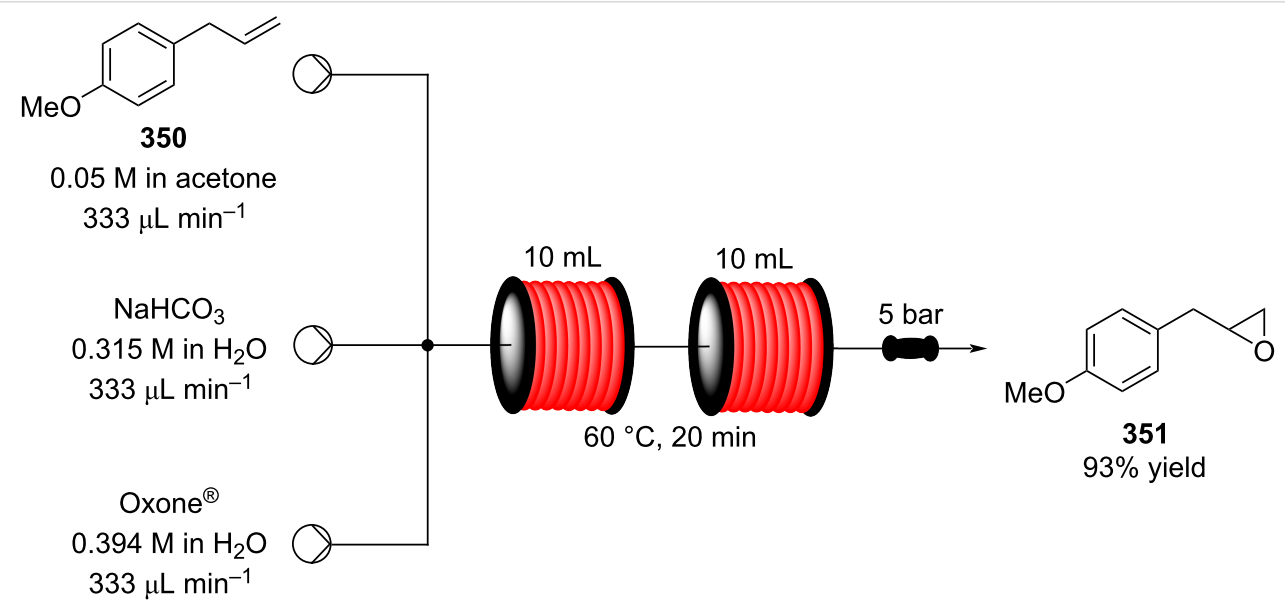<smiles>c1ccc(CC2CO2)cc1</smiles>

352

98\% yield<smiles>O=C1c2ccccc2C(=O)C2OC12</smiles>

355

$74 \%$ yield<smiles>O=C(O)c1ccc(CC2CC2)cc1</smiles>

353

$65 \%$ yield<smiles>BrCC1CO1</smiles>

356

$90 \%$ yield<smiles>OCC1OC1c1ccccc1</smiles>

354

$60 \%$ yield<smiles>CC(=O)C1OC1(C)C</smiles>

357

$90 \%$ yield

Scheme 84: Continuous-flow epoxidation using DMDO as oxidant.

epoxides were then employed for creating a compound library of potential new pharmaceuticals but could be used in many different applications.

Mukayama-type aerobic epoxidation has also been implemented in flow mode by Favre-Réguillon and his group [335]. A segmented flow regime increases the mass transfer and allowed a reduction in the required reaction time to 5 minutes (Scheme 85). Additionally, the system requires lower catalyst loading compared to the batch approach $(0.1 \mathrm{~mol} \%$ vs 100 ppm) and quantitative conversion and high selectivity (>99\%). The same system was also explored for the epoxidation of several fatty acids [336].

Various zeolite supports have found use as heterogeneous catalysts in flow through filled reactor cartridges and channels. Wan et al. used a titanium silicate zeolite micro-engineered reactor for the epoxidation of 1-pentene in flow [337]. However, the

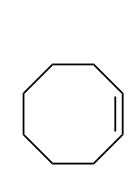

257

$0.67 \mathrm{M}$ in heptane 2-ethylhexanal (3 equiv) $\mathrm{Mn}$ (II) catalyst (100 ppm)

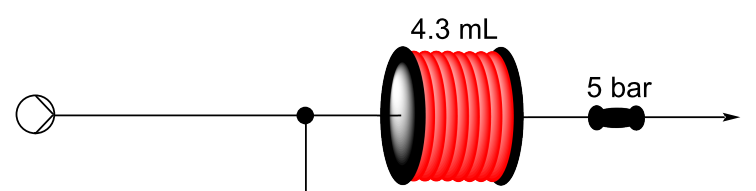

$\mathrm{rt}, 5 \mathrm{~min}$

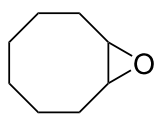

358

$100 \%$ conversion $>99 \%$ selectivity 
system showed deactivation over prolonged usage equating to $\approx 100$ hours $(\approx 500 \mathrm{mg}$ of starting material), meaning the catalyst had to be eventually replaced. Similarly, Okrut et al. developed a crystalline Ti-based zeolite for the epoxidation of 1-octene (650) with ethylbenzene hydroperoxide (EBHP) [338]. The catalyst was designed as a valid replacement for the silica supported Ti-based catalyst employed from Shell in propylene oxide preparation [339]. The Shell catalyst allows to convert the alkene up to $84 \%$ and higher temperatures are required to complete the transformation, leading to reduced catalyst selectivity and deactivation. The zeolite-based Ti catalyst proved to be more robust and active than the amorphous silica-supported one, showing no evidence of deactivation over 72 hours of runtime [338]. After use, the zeolite can be calcinated to regenerate its activity.

In 2015, Chen and Gao et al. demonstrated one of the few asymmetric epoxidations in flow exploiting a previously described biomimetic iron catalyst with ligand $\mathbf{3 6 0}$ (Scheme 86) [340,341].

The system was tested on several electron-deficient olefin derivatives 359 and the corresponding epoxides 361 were obtained in good to excellent yields (52-90\%) and moderate to good selectivity (63-92\% ee). The apparatus presented some advantages over the batch reaction such as reduced reaction times ( 2 minutes vs 2 hours) and decreased peroxyacid equivalents ( 1.25 equiv vs 2 equiv).
A micro-structured PEEK (polyether ether ketone) reactor was developed in 2009 for the enantioselective epoxidation of chalcone (235), catalysed by poly-L-leucine [342]. A staggered herringbone micromixer design generated efficient mixing, however, productivity amounted to only $0.5 \mathrm{~g}_{\text {day }}{ }^{-1}$ due to the small reactor dimensions. In the same year, Watts and Wiles et al. developed a continuous-flow system using a commercially available lipase supported on a macroporous acrylic polymer (Novozyme ${ }^{\circledR} 435$ ) for epoxidation [343]. In this example, as in many others, the authors demonstrated the use of flow allows for intensified reaction conditions to be used, increasing throughput and beneficially preventing enzyme deactivation (Scheme 87). Indeed, higher concentrations of hydrogen peroxide were tolerated $(0.3 \mathrm{M}$ vs $0.05 \mathrm{M})$ alongside higher reaction temperatures ( $\mathrm{rt}$ vs $70{ }^{\circ} \mathrm{C}$ ). The system could be efficiently operated for over 25 hours without any detrimental effects.

Ozonolysis is a related electrophilic oxygenation process which carries with its huge potential safety benefits with regards to handling explosive and hazardous reaction intermediates meaning likewise it is highly beneficial to perform in flow. In 2006, Jensen and his group developed an engineered multichannel microreactor for the ozonolysis of 1-decene (366) [344]. The reactor was fabricated in Pyrex ${ }^{\circledR}$ with silicon posts placed in the channels to increase the pressure drop, and allow liquid-gas interactions. The apparatus was explored for the oxidations of three different functional groups; phosphites,

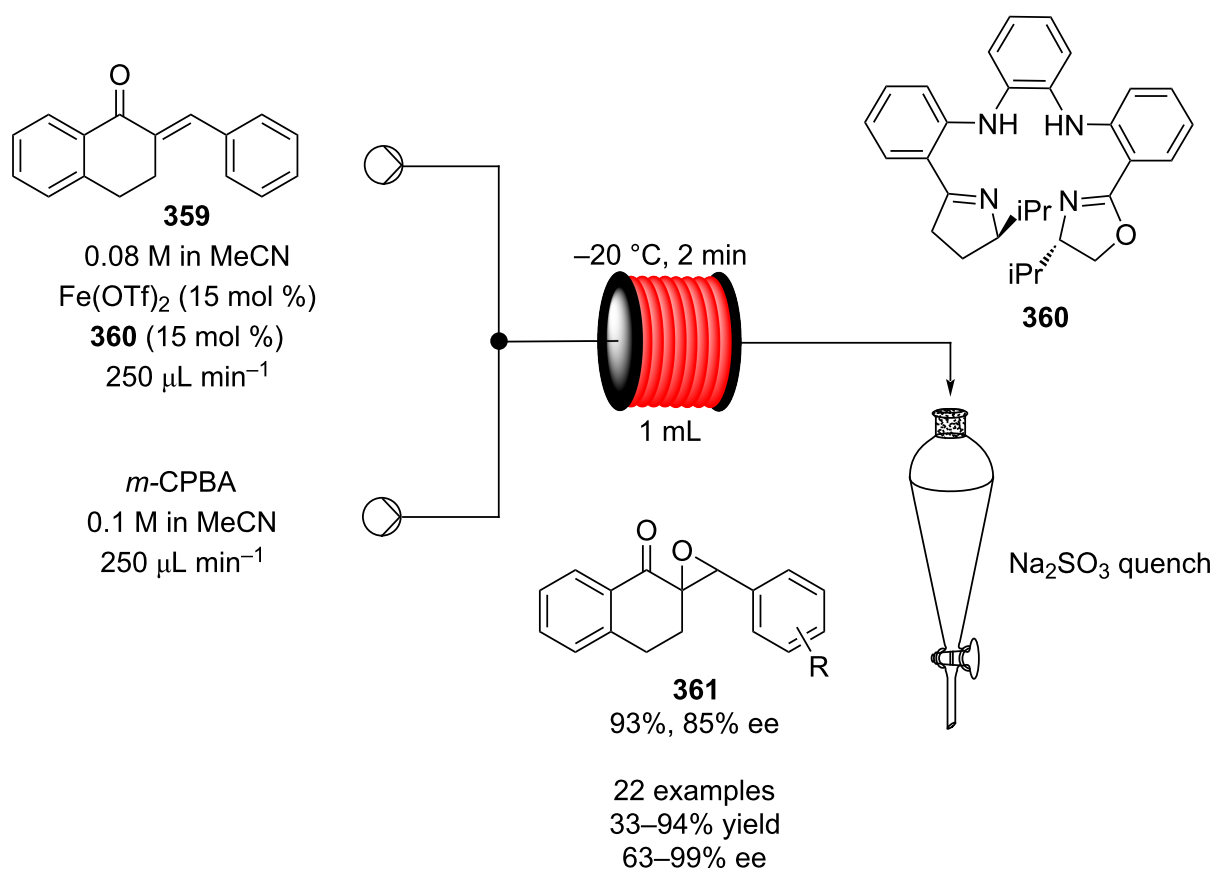

Scheme 86: Continuous-flow asymmetric epoxidation of derivatives of 359 exploiting a biomimetic iron catalyst. 


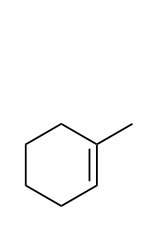

362

$0.1 \mathrm{M}$ in AcOEt

$\mathrm{H}_{2} \mathrm{O}_{2}$ (2 equiv)

$10 \mu \mathrm{L} \min ^{-1}$

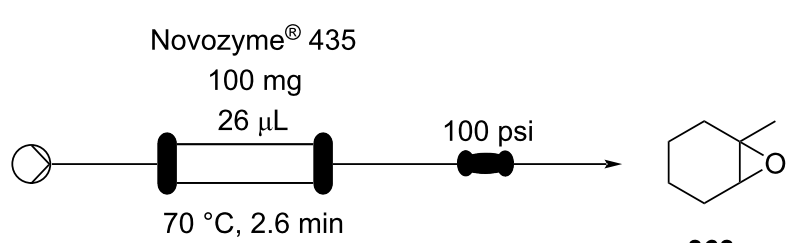

363

$99.1 \%$ yield

throughput $6.7 \mathrm{mg} \mathrm{h}^{-1}$

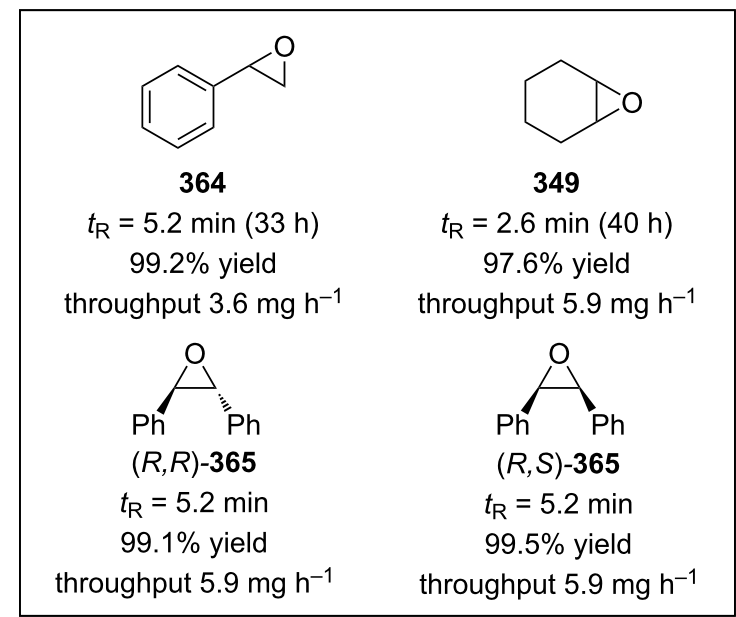

Scheme 87: Continuous-flow enzymatic epoxidation of alkenes developed by Watts et al.

amines, and alkenes. The authors pointed out a residence time as short as 0.32 seconds was enough to efficiently convert 1-decene (366) to nonanal (345, Scheme 88).

Jähnisch and co-workers also developed a micro-structured device for ozonolysis, which was employed for the preparation of a vitamin D precursor, 368 [345]. The system exploited a 5-channel micromixer called "cyclone", which allows a high mixing effect increasing the flow rate. The ozolonysis step was coupled with a reductive one and the system could produce $1.22 \mathrm{~kg}$ of desired material per day in $69 \%$ overall isolated yield. The same group also developed the ozonolysis for

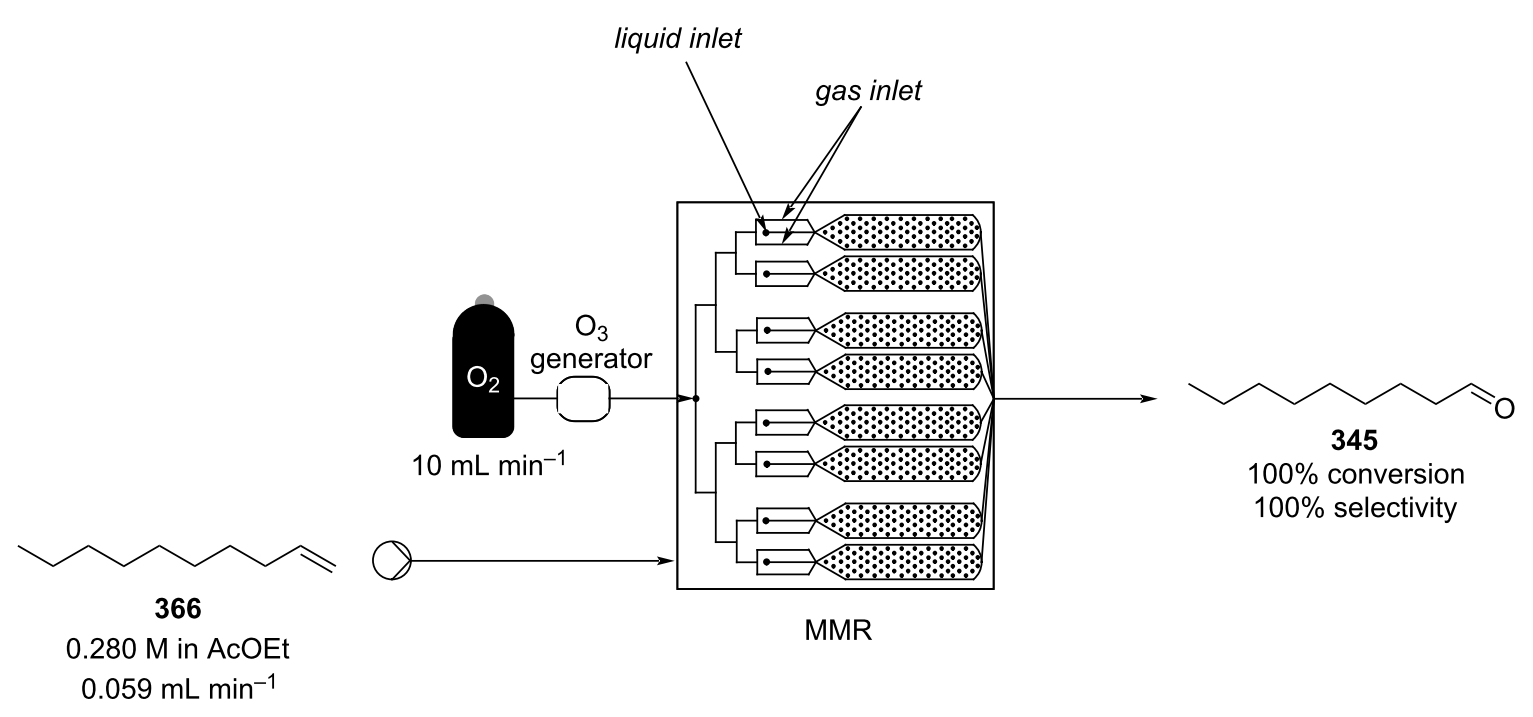

Scheme 88: Engineered multichannel microreactor for continuous-flow ozonolysis of $\mathbf{3 6 6 .}$ 
1-decene (366) and acetic acid 1-vinyl ester using a falling-film microreactor (FFMR) [346,347]. To monitor the conversion of the reaction, all these experiments were monitored using an on-line FTIR with an attenuated total reflectance (ATR) sensor (Scheme 89).

The general flow setup shown in Scheme 90 was used by Kappe et al. for the ozonolysis of various compounds, affording products 47, 264, and 344-348 all in good yields [348]. The ozonolysis was performed using a ThalesNano ${ }^{\circledR}$ device called the $\mathrm{O}_{-C u b e}{ }^{\mathrm{TM}}$. Ozone was generated in-line from electrolysis of $\mathrm{O}_{2}$ and was delivered through a PTFE frit to a precooled stream of starting material solution. The mixture was then run through a second reactor coil before being met by a stream of quenching solution. Conditions used were substrate specific and tuning of temperature and quenching solution could be used to achieve selectivity between sulfone $\mathbf{3 4 9}$ and sulfoxide $\mathbf{3 5 0}$.

In 2010, Ley and his group exploited a semi-permeable material (Teflon AF-2400) to build a simple and efficient flow apparatus for ozonolysis [349]. A coil of semi-permeable material was inserted into a glass bottle where ozone was flushed in, and a stream of alkene solution was pumped through the coil. The system was capable of oxidising different alkenes at room temperature in moderate to excellent yields and few examples are depicted in Scheme 91. After collection, the so formed ozonides were quenched using polymer-supported triphenylphosphine $\left(\mathrm{PS}-\mathrm{PPh}_{3}\right)$.

In 2016, Tyler and his group explored the use of a film-shear flow reactor to enhance liquid-gas interaction [350]. The authors pointed out the possibility to achieve a high throughput, as the residence time was only a few seconds, and helped to avoid falling-film microreactor and the need for stoichiometric reducers. In fact, the system employed water as a mild reductant (Scheme 92), following a modification described by Dussault and Schiaffo [351]. The ketones and aldehydes formed could be easily isolated from the organic phase. However, this flow methodology is not applicable for alkylalkenes as they produce too unstable Criegee intermediates, which rapidly decompose to the ozonide.

Significant work in this area by Gavriilidis and co-workers [352,353] and Sun et al. [354] has further demonstrated the potential of performing ozonolysis reactions and handling their intermediates in flow. In 2017, Subramaniam et al. used static mixer to enhance mixing for the ozonolysis of several fatty acid esters [355]. They confirmed that $\mathrm{scCO}_{2}$ could be used as the solvent, and additionally, the authors developed a CSTR for continuous ozonide decomposition. This system consists of a Parr reactor set to a certain temperature, which was continuously fed by a stream of ozonide reaction mixture. For safety reasons, the reactor was kept under nitrogen atmosphere and filled only one third of its full capacity. After 20 minutes of residence time, the outlet stream was collected in an open container to vent the generated gas. The authors investigated a large range of temperatures $\left(80\right.$ to $150{ }^{\circ} \mathrm{C}$ ) finding highest selectivity at $80{ }^{\circ} \mathrm{C}$.

As flow chemistry facilitates the use of in-line analysis, different mechanistic studies on ozone-driven oxidations of alkene compounds in air have been performed, leading to interesting

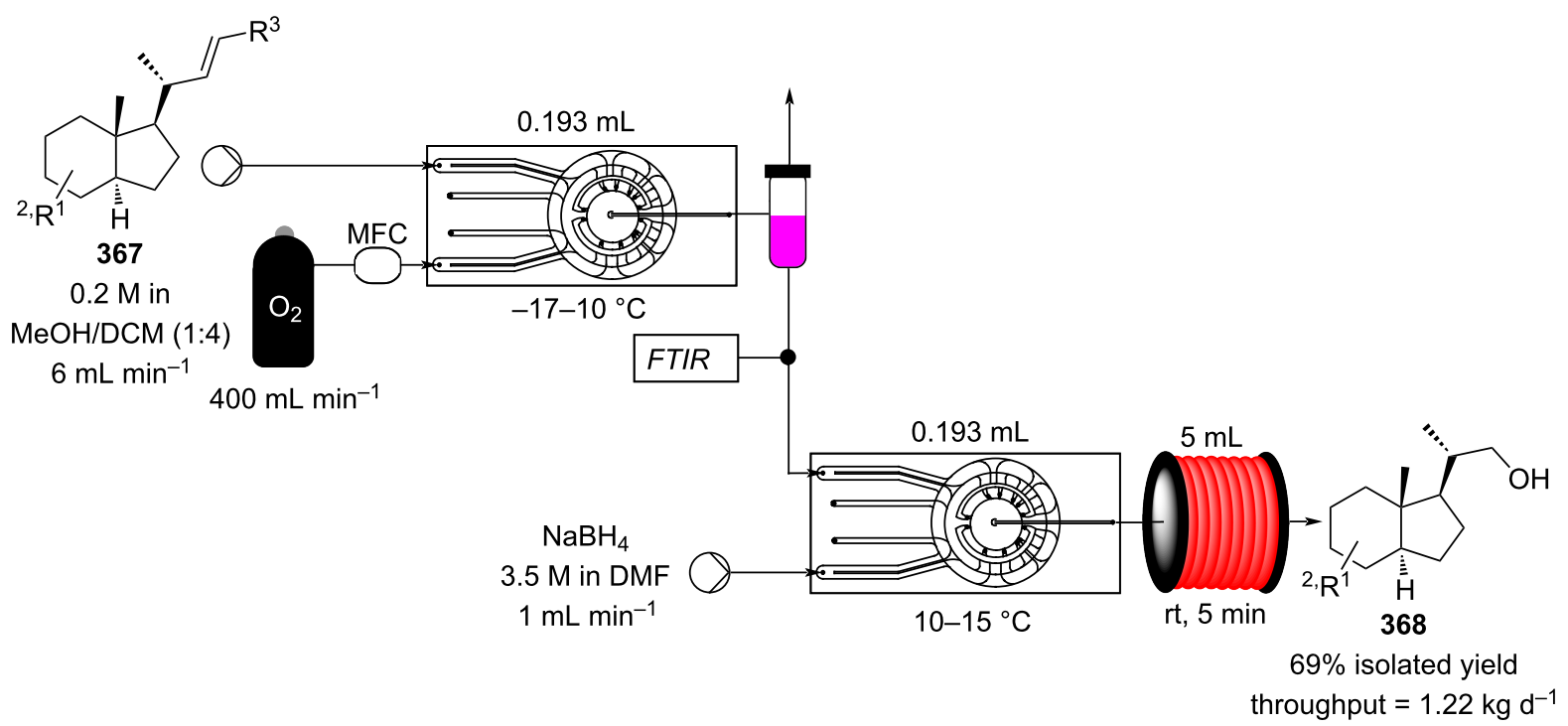

Scheme 89: Continuous-flow synthesis of the vitamin D precursor 368 using multichannel microreactors. MFC: mass flow controller. 


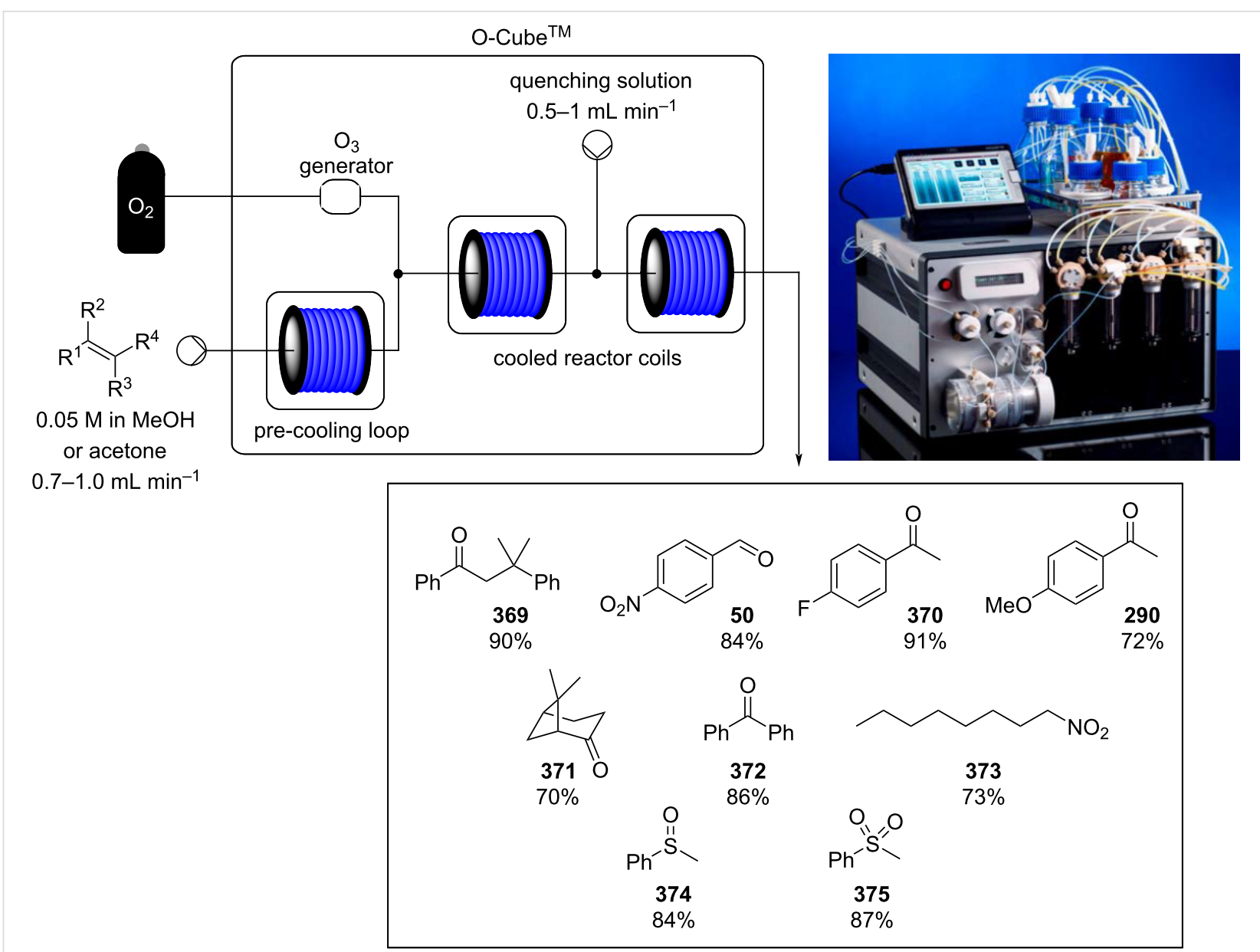

Scheme 90: Continuous ozonolysis setup used by Kappe et al. for the synthesis of various substrates employing ThalesNano ${ }^{\circledR}$ O-Cube ${ }^{\mathrm{TM}}(\mathbf{5 0}, \mathbf{2 9 0}$, 369-375) [348]. Reprinted with permission from [348]. Copyright 2011 American Chemical Society.

results difficult to be obtained without a flow approach $[62,77,356-358]$.

An alternative oxidative cleavage of olefins can be achieved through the use of molecular oxygen itself as the sole oxidant in a process catalysed by $\mathrm{Pd}^{\mathrm{II}}$ [359]. Improvements to the process have been obtained with a flow reactor system compared with the batch equivalent decreasing the time of reaction and the catalyst loading. Undoubtedly, the incorporation of flow technology into transformations requiring oxygen as well as other gaseous reagents will become increasingly popular for the reasons discussed above.

Organosulfur compounds such as ajoene (386) and allicin (388) are key components in onion and garlic extracts. These products provide odour and some therapeutic benefits to these herbs, and consequently are important materials in F\&F and pharmaceutical industry [360]. Different procedures for their synthesis have been disclosed so far and sulfide oxidation is described as the key step (Scheme 93) [361-364]. For instance, ajoene (386) can be prepared by hydrolysis and sulfenylation of the thioenaloate starting material $\mathbf{3 8 4}$ to prepare the intermediate $\mathbf{3 8 5}$, which can then be oxidised to the desired material 386. Allicin (388) could be prepared by oxidation of diallyl disulphide (387).

Over the years, many oxidising agents and conditions have been tested to selectively convert sulfides to the corresponding sulfoxides. However, due to their propensity for overoxidation, exact control of the reaction time and oxidant stoichiometry is of paramount importance to avoid sulfone formation, hence flow chemistry has been evaluated as a processing solution. While pursuing this work, a review on this subject has also been published by Colomer and co-workers [365]. In this area Doherty et al. have published widely on the application of peroxotungstates being immobilised on ionic resins that allow for selective oxidisation along with a low catalyst leaching. The application in flow enabled a lower catalyst loading and reduced residence times (Scheme 94) [366,367]. 

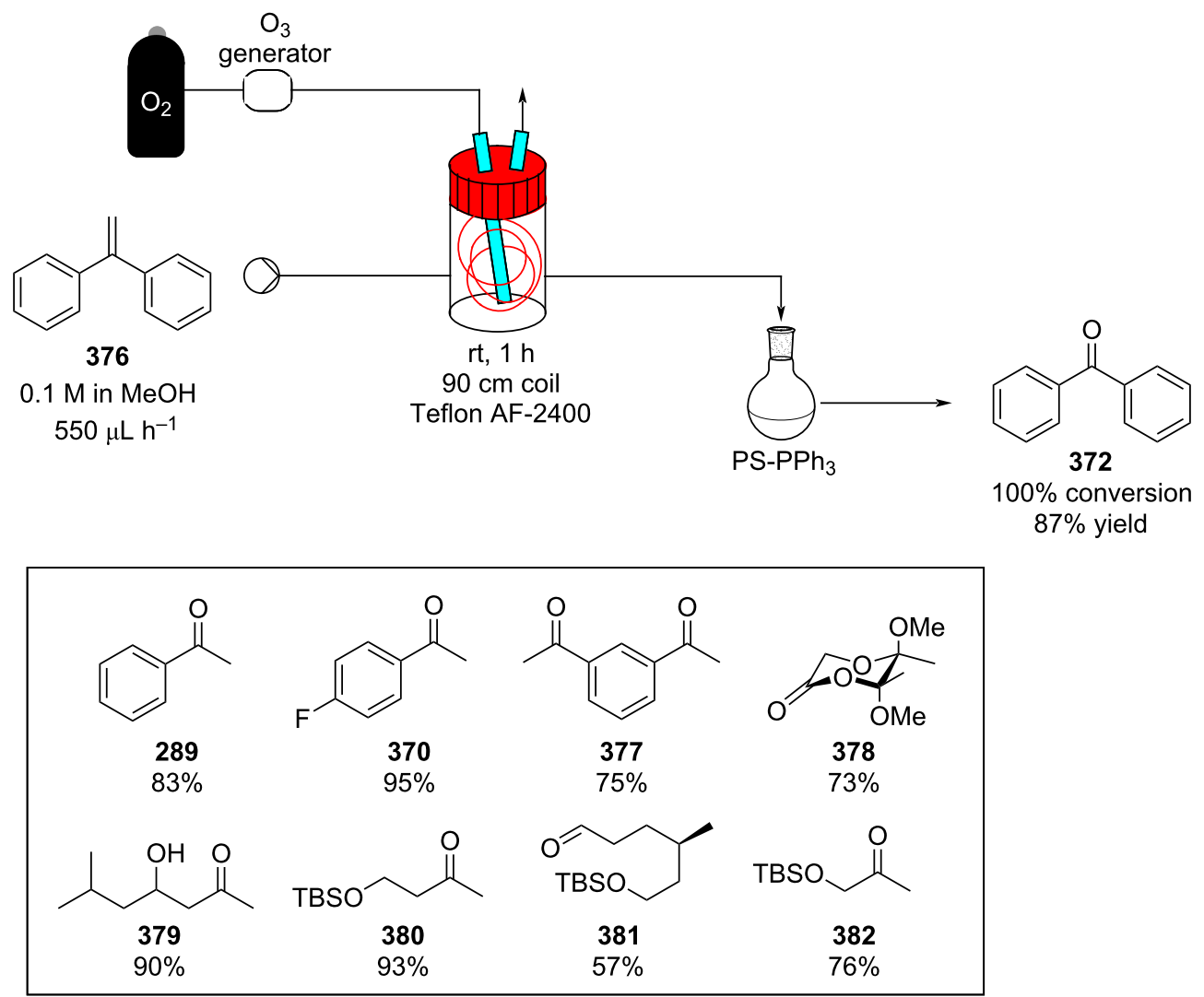

Scheme 91: Continuous-flow apparatus for ozonolysis as developed by Ley et al.

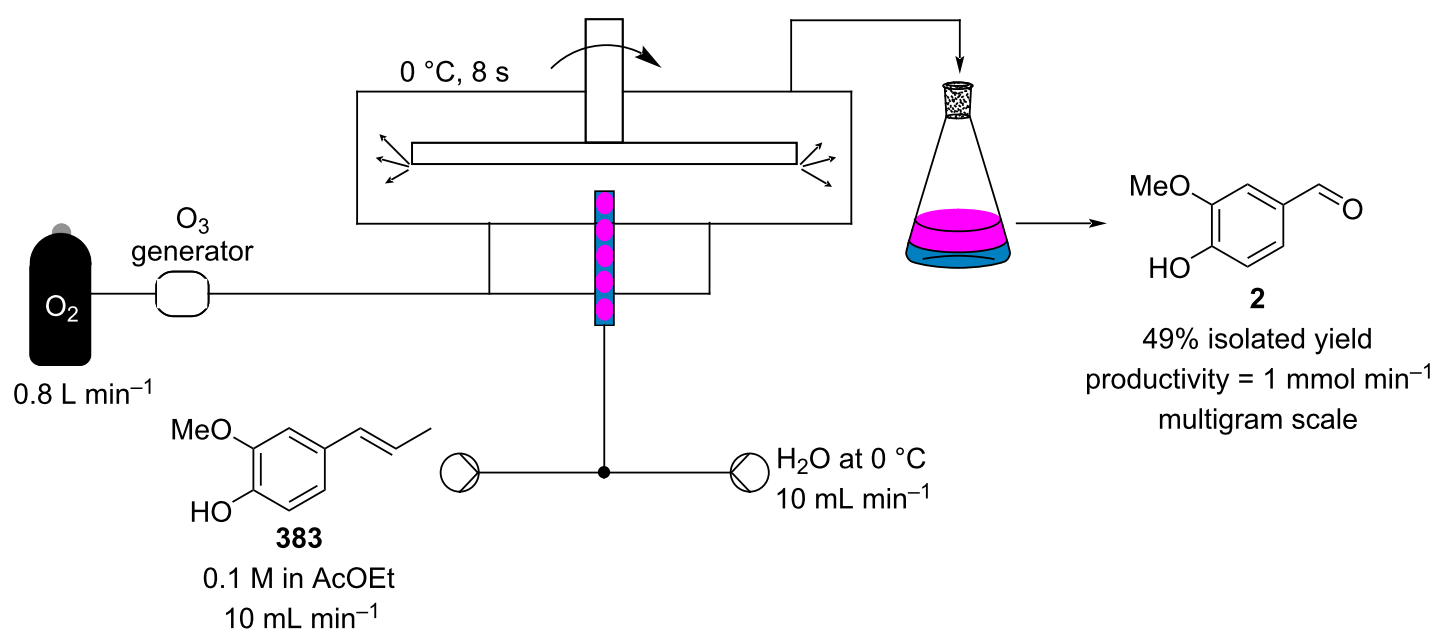

Scheme 92: Continuous-flow ozonolysis for synthesis of vanillin (2) using a film-shear flow reactor.

Along with the sulfoxides, thiosulfinates have been synthesised in flow using a packed-bed reactor filled with oxone as described by Wirth et al. (Scheme 95) [368]. This approach has been used to prepare very reactive metabolic sulfoxides and sulfenic acids present in garlic at increased scale.
Electrochemical reactions have various disadvantages which prevent them from being widely performed on scale. Organic decomposition on the electrode, and hot-spot formation reduce the performance over the time and limit process scalability. The usage of flow technology could overcome such issues. 

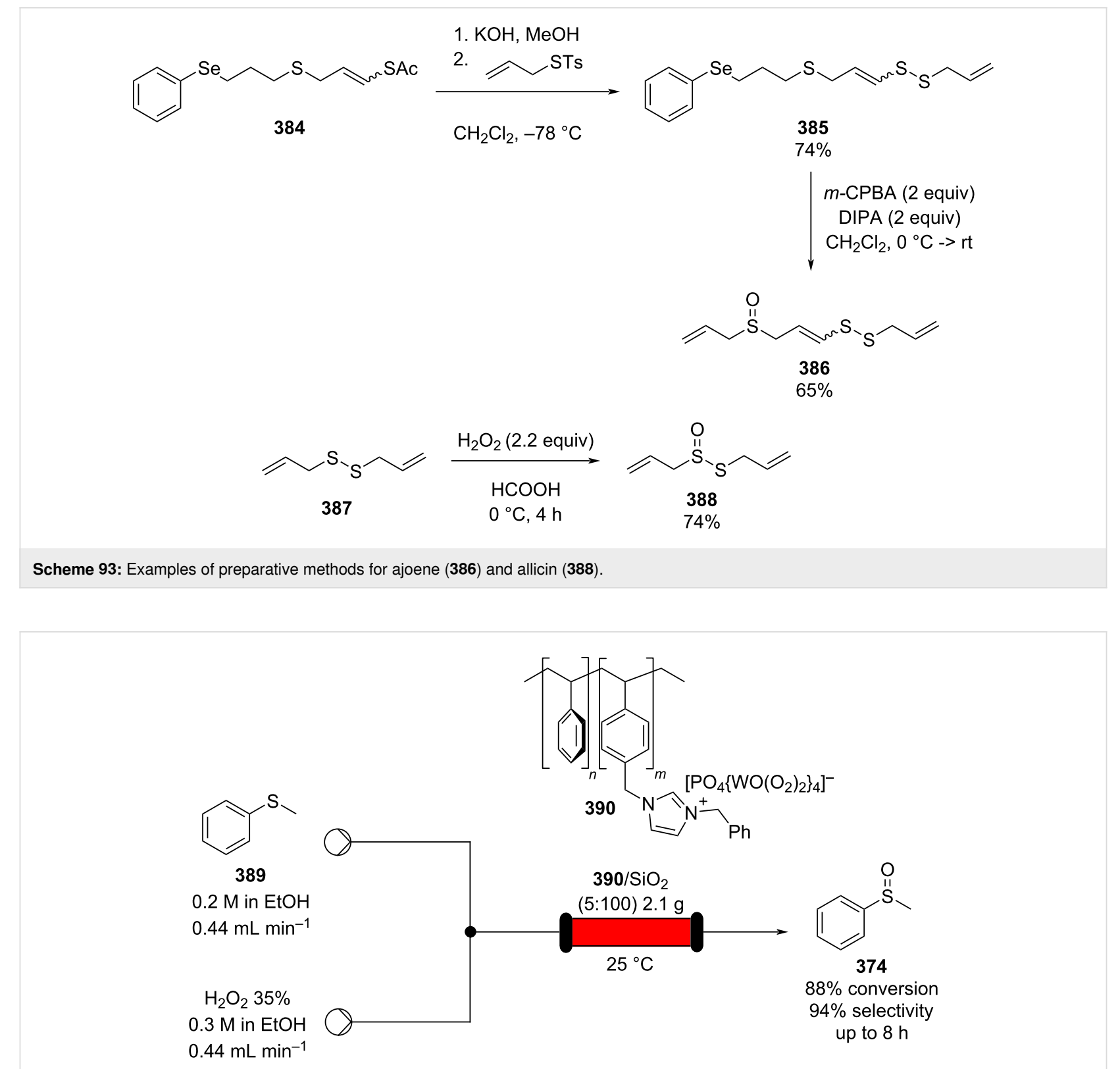

Scheme 94: Continuous-flow oxidation of thioanisole (389) using styrene-based polymer-supported peroxytungstate catalyst 390.

In 2017, Noël et al. assembled a microflow electrocell for the electrochemical oxidation of thioether compounds. This apparatus enable the avoidance of stoichiometric oxidising agents, and reduced reaction times (Scheme 96) [369]. Fine tuning the voltage and reaction times of the flow system, allowed 17 different substrates to be oxidised to their corresponding sulfoxides or sulfones in moderate to good yields $(31-92 \%)$.

Recent industrial interest in establishing continuous oxidation processes is demonstrated by the rapidly increasing patent literature, for example, for the synthesis of hexanoic acid from 2-octanol [370] and the microreactor-based continuous aerobic oxidation to chalcone (235, Scheme 97) [371].

In 2018, IFF, patented a continuous-flow process for the oxidation of methyl dihydrojasmonate (33) through a two-step chlorination-elimination reaction. The system enabled the efficiently synthesis the desired dehydrated material $\mathbf{4 0 1}$ in $72 \%$ yield safely using sulfuryl chloride as the chlorinating agent (Scheme 98) [372]. The chlorination step was carried out in an Uniqsis FlowSyn reactor and, after collection and $\mathrm{MeOH}$ addition, the elimination step occurred spontaneously in batch over a period of 16 hours. 


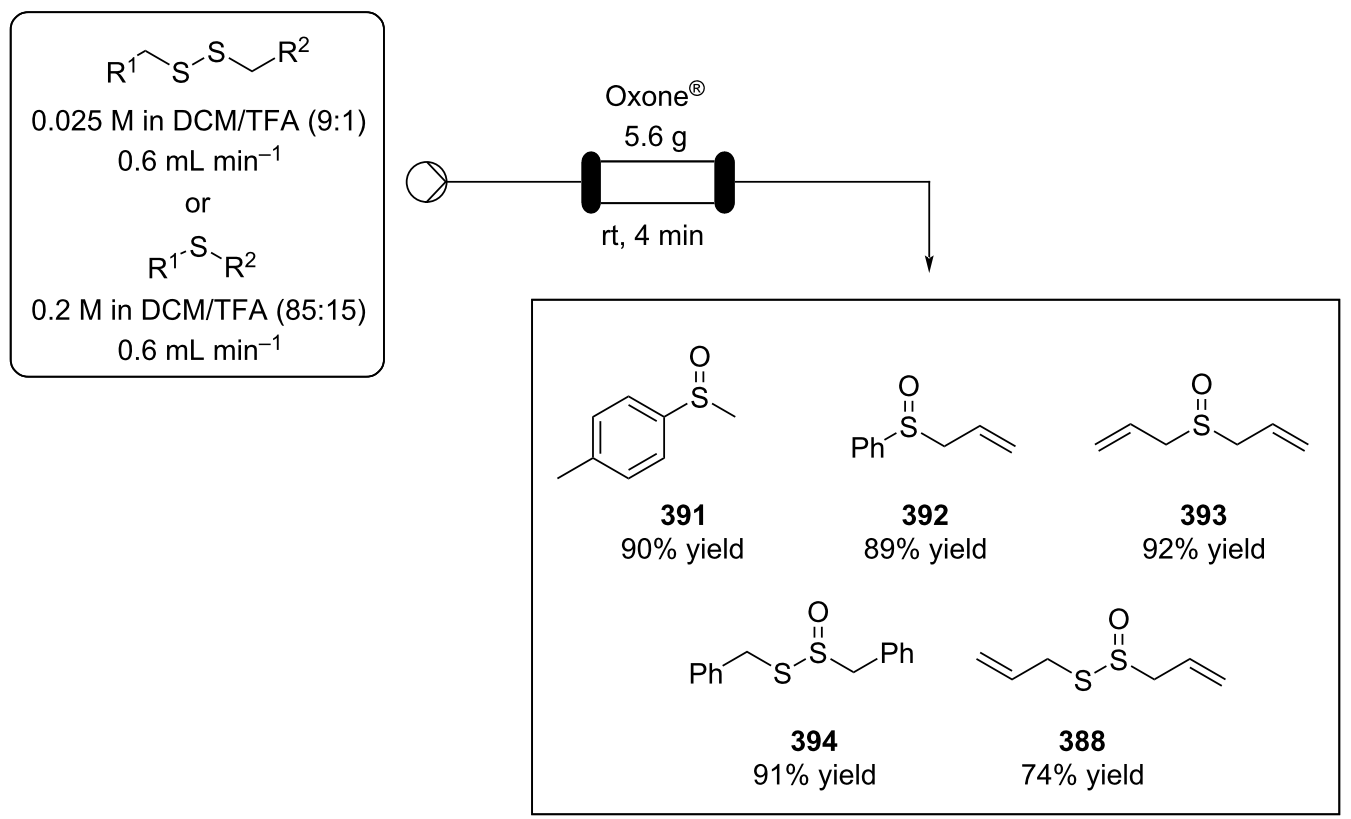

Scheme 95: Continuous oxidation of thiosulfinates using Oxone ${ }^{\circledR}$-packed reactor.
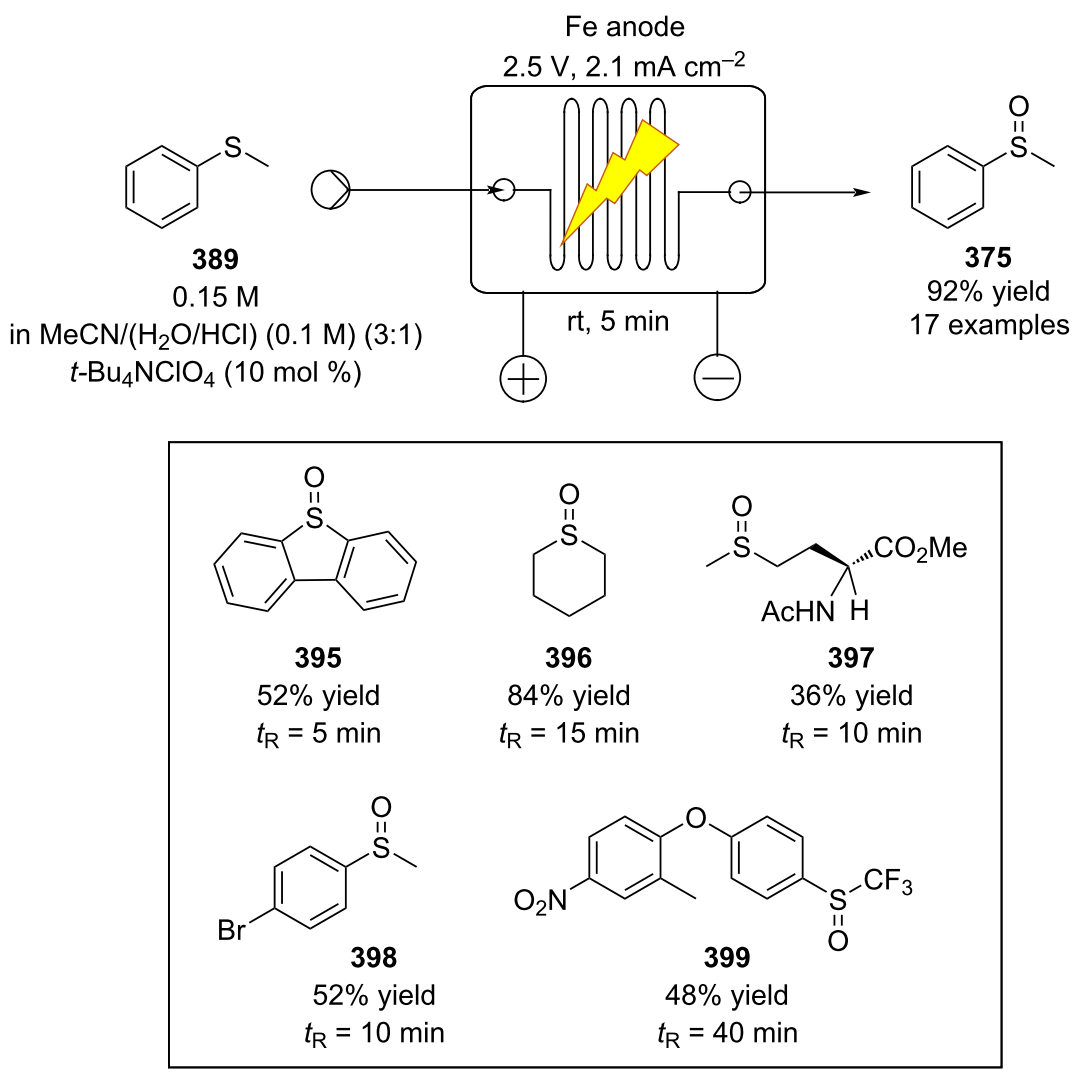

Scheme 96: Continuous-flow electrochemical oxidation of thioethers. 


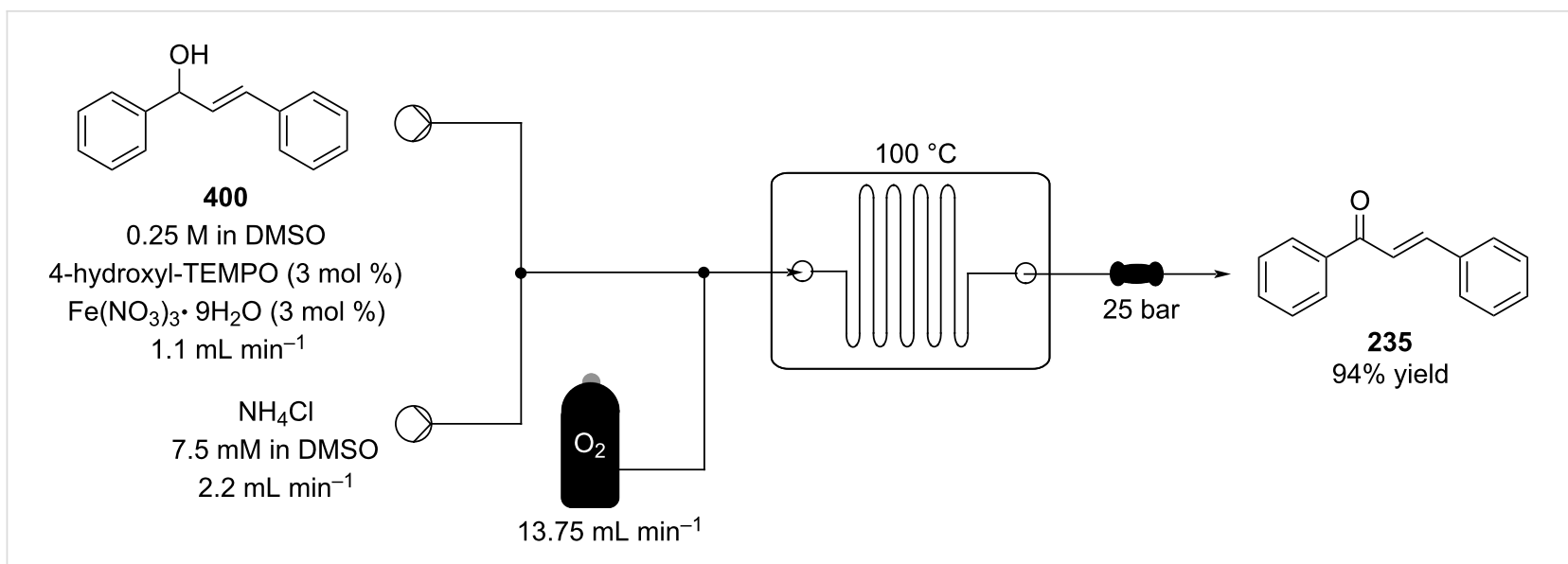

Scheme 97: Continuous-flow oxidation of $\mathbf{4 0 0}$ to cinnamophenone (235).

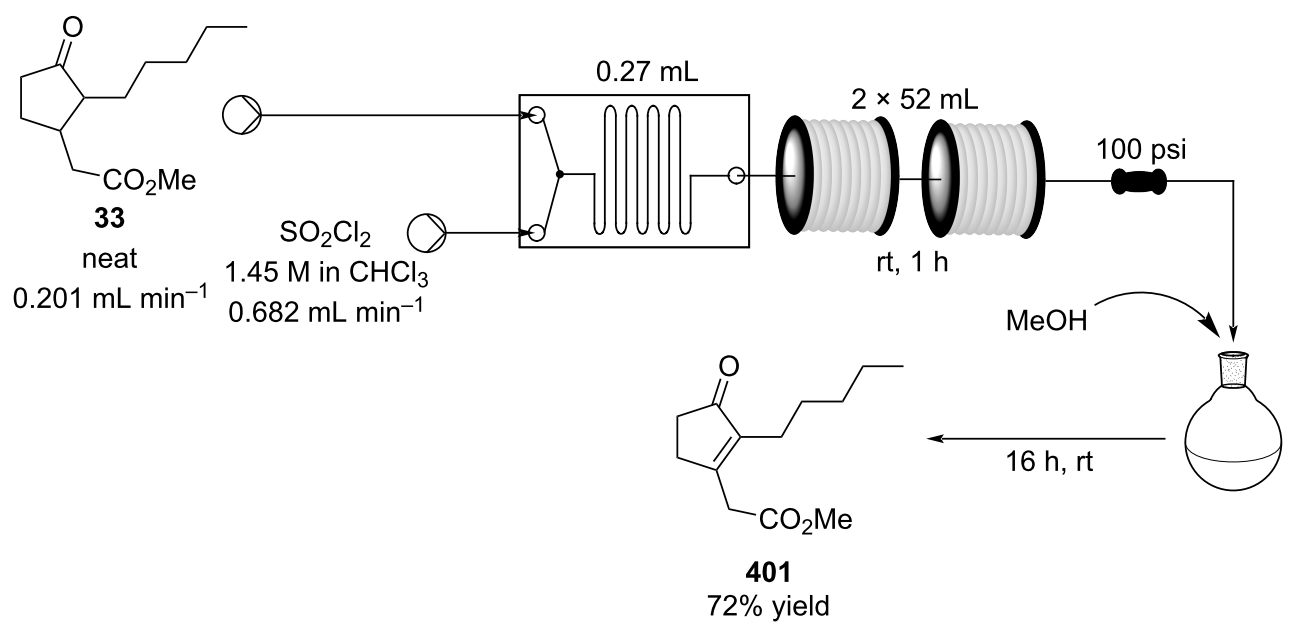

Scheme 98: Continuous-flow synthesis of dehydrated material 401 via oxidation of methyl dihydrojasmonate (33).

\section{The use of Grignard reagents}

The Grignard reaction is a particularly important $\mathrm{C}-\mathrm{C}$ bond forming sequence for the synthesis of higher order alcohol products and intermediates. Pertinent examples relating to the fragrance and flavours industry include the methylation of 6-methyl-5-hepten-2-one (210) [373] and for the introduction of vinyl groups to 2-chloro-5-cyclohexadecen-1-one (404), in a synthesis of velvione (407) (Scheme 99) [374]. Grignard reagents are also commonly employed as strong bases, for example, in the aldol reactions of cyclohexenyl methyl ketones, key intermediates in the synthesis of $\alpha$-, $\beta$ - and $\delta$-damascones (412) $[375,376]$. These structures represent a very reactive class of compounds that require strict control over the conditions including an inert environment and are renowned for possessing highly exothermic reaction profiles. The application of flow to the use and preparation of Grignard reagents has been explored fairly extensively in the past few years.
Industrially, Grignard reagents are mainly prepared in stirred tank reactors via halogen/magnesium insertion reaction. The process can be highly exothermic and can therefore be problematic as the ideal preparative solvents are normally ethers which have typically low boiling points and are highly flammable. Moreover, heat removal during activation can be difficult and inhomogeneous hot spots readily occur inducing side reactions (i.e., Wurtz coupling), reducing the efficacy of the preparation and increasing associated risk at scale.

In 2012, a continuous preparation of Grignard reagents was performed using a heated column with a multi-bladed stirrer filled with granulated magnesium (Scheme 100) [377]. The halide was fed from the bottom as a mixture in ether (diethyl ether or THF) and toluene. The organomagnesium halide compound stream was collected from the top of the reactor. The magnesium was first activated by introducing a mixture of ether and 


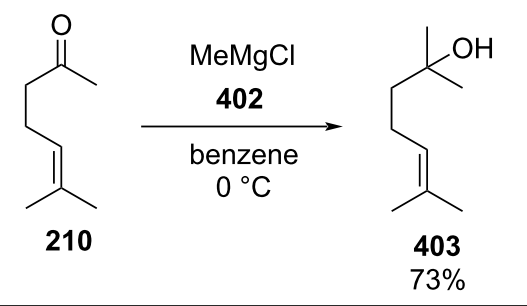<smiles>O=C1CCCCCCCCCCC1Cl</smiles>

404

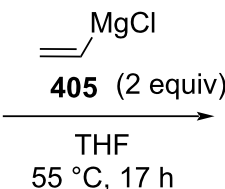

$55^{\circ} \mathrm{C}, 17 \mathrm{~h}$<smiles>C=CC(CCCCCCCCCCC)C(O)(C=C)CC</smiles>

406

$99 \%$

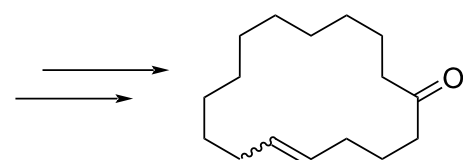

407<smiles>CC(=O)C1=C(C)C=CCC1(C)C</smiles>

408

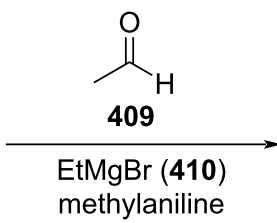

THF/toluene $10-15^{\circ} \mathrm{C}$-> rt, $90 \mathrm{~min}$<smiles>C[PH+]CC(O)CC(=O)C1CCCCC1(C)C</smiles>

411

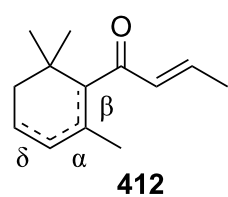

$73 \%$

Scheme 99: Some industrially important transformations involving Grignard reagents.

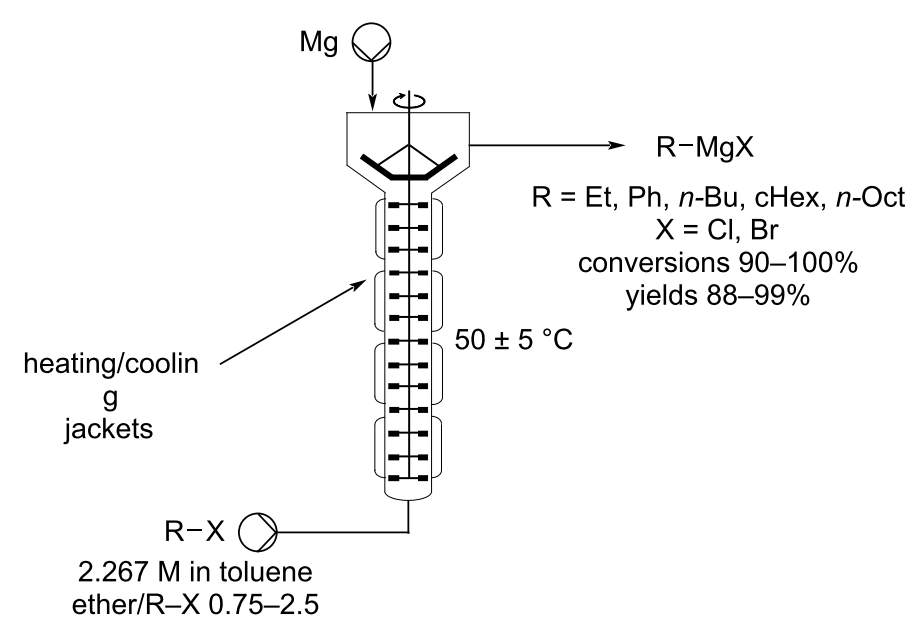

Scheme 100: Grachev et al. apparatus for continuous preparation of Grignard reagents.

dibromoethane in toluene and then a $2.267 \mathrm{M}$ solution of alkyl halide was constantly introduced. The preparation of different halides was optimised using ether/R-X ratios from 0.75 and 2.5 gaining excellent conversions (90-100\%) and excellent yields $(88-99 \%)$.

A few years later, the group of Duchateau also reported a continuous preparation of phenylmagnesium bromide using a magnesium fluid bed reactor (Scheme 101) [378].
Granular magnesium was loaded into a $75 \mathrm{~mL}$ stainless steel tube reactor and activated by flushing with a premade solution of the Grignard reagents in THF. A 1 M solution of bromobenzene in THF or cyclopentyl methyl ether (CPME) was pumped through the tube reactor at a settling velocity to allow the solid to fluidize into the flow stream. Instead of titrating, the air-sensitive magnesium halide was monitored using an on-line $42 \mathrm{MHz}$ NMR spectrometer. A screening of different reaction conditions enabled optimisation to ensure complete 


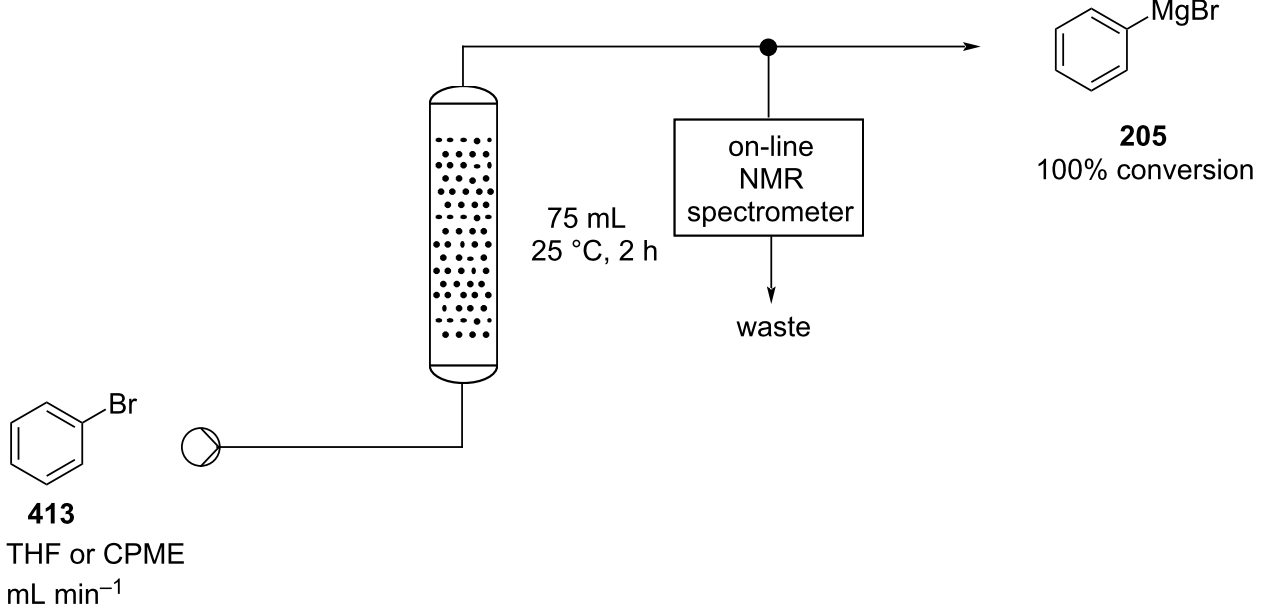

Scheme 101: Example of fluidized Mg bed reactor with NMR spectrometer as on-line monitoring system.

conversion of the halide input at $25{ }^{\circ} \mathrm{C}$ with a 2 hours residence time.

Alcázar et al. also used this approach to generate a stream of Grignard reagents from a column reactor (magnesium particle size 20-230 mesh) [379]. The solid packed reactor was initially flushed with a solution of DIBAL-H in THF/toluene (1:1) and then activated by flowing TMSCl and 1-chloro-2-bromoethane in toluene through the system. The Grignard reaction (section of aryl and alkyl halides) was then performed in the presence of
$\mathrm{LiCl}(0.5 \mathrm{M})$ in THF to avoid clogging of the column due to solid formation. After 7.5 minutes a solution $0.38 \mathrm{M}$ of the organomagnesium halide was collected (estimated metallic composition $76 \%$ ). On larger scales this performance did not change until $60-70 \%$ of the amount of magnesium had been consumed. Several examples of Grignard reagents were synthesised through this method and these were then directly mixed in-line with electrophiles gaining different addition products in good to excellent yields (Table 8). The freshly-made Grignards were also collected and employed in other reactions where the

Table 8: Continuous synthesis of Grignard reagents and subsequent quenching with electrophiles.
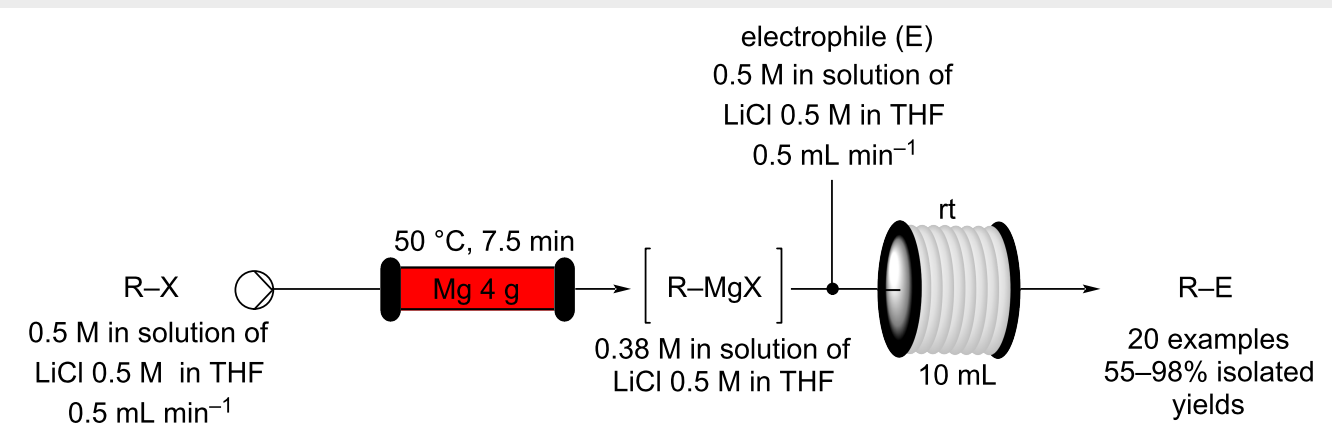

Entry Electrophile Product


Table 8: Continuous synthesis of Grignard reagents and subsequent quenching with electrophiles. (continued)

3<smiles>CON(C)C(=O)C(C)C(C)(C)Cl</smiles>

417

4<smiles>O=C=Nc1ccccc1</smiles>

419

5<smiles>O=Cc1cccnc1</smiles>

421

6<smiles>O=Cc1ccccc1</smiles>

7<smiles>O=Cc1ccccc1</smiles>

17

8<smiles>O=Cc1ccccc1</smiles>

17<smiles>Brc1ccccc1</smiles>

413<smiles>Brc1ccccc1</smiles>

413<smiles>Clc1cccc(Br)c1</smiles>

422<smiles>COc1ncccc1Br</smiles>

$n-\mathrm{Bu}-\mathrm{MgBr}$

426<smiles>CN1CCC(Cl)CC1</smiles><smiles>O=C(Cl)C(=O)c1ccccc1</smiles>

418<smiles>O=C(Nc1ccccc1)c1ccccc1</smiles>

420<smiles>OC(c1cccnc1)c1cccc(Cl)c1</smiles>

423<smiles>COc1ncccc1C(O)c1ccccc1</smiles>

75<smiles>CCCCC(O)c1ccccc1</smiles>

98

427<smiles>CN1CCC(C(O)c2ccccc2)CC1</smiles>

429 batch protocol is more suitable such as conjugate addition, sulfone formation and Negishi couplings. More recently, Noël et al. described a similar telescoped system for the in situ preparation of organomagnesium bromides to be employed in the iron-catalysed cross-coupling with alkenyl and styrenyl chlorides [380].

In 2010, a collection of secondary and tertiary alcohols, including the analgesic tramadol (anti-443), were prepared from their corresponding ketones in flow (Scheme 102) [381].
After merging the starting material streams at room temperature and passage through a series of reactor coils the mixture was directed through a packed column containing a polymersupported benzaldehyde resin which was used to scavenge any excess Grignard reagent. This yielded after a batch quenching step and work-up the alcohol products in high yields (Table 9).

Significant effort has been directed towards in-line work-ups for flow protocols involving Grignard reagents as a safer way of

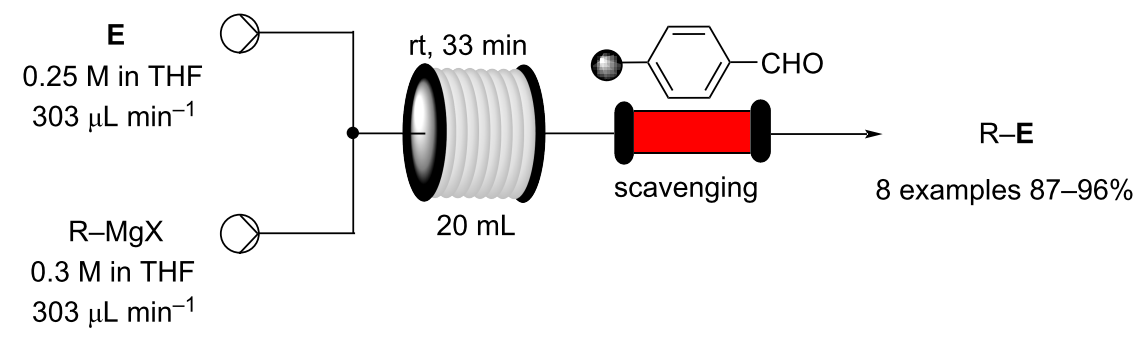

Scheme 102: Continuous-flow synthesis of Grignard reagents and subsequent quenching reaction. 
Table 9: Continuous-flow reactions of aromatic aldehydes/ketones with Grignard reagents and substrates synthesised.

\begin{tabular}{|c|c|c|c|c|}
\hline Entry & Electrophile & $\mathrm{R}-\mathrm{MgX}$ & Product & Yield (\%) \\
\hline 1 & 27 & 430 & 431 & 92 \\
\hline 2 & 27 & 432 & 433 & 93 \\
\hline 3 & 27 & 434 & 435 & 87 \\
\hline 4 & 289 & 430 & 436 & 94 \\
\hline 5 & 289 & 432 & 437 & 95 \\
\hline 6 & 289 & 434 & 438 & 90 \\
\hline 7 & 289 & $\begin{array}{c}\mathrm{MeMgBr} \\
439\end{array}$ & 440 & 95 \\
\hline 8 & 441 & $\begin{array}{l}\text { OMe } \\
\quad 442\end{array}$ & $\begin{array}{l}\mathrm{Me} \\
\text { anti-443 }\end{array}$ & $\begin{array}{c}96 \\
\operatorname{dr}(\text { syn/anti) } \\
2: 8\end{array}$ \\
\hline
\end{tabular}

quenching the reaction and reducing the decomposition of the intermediate metallated species. A continuous-flow approach to 3,3,3-trifluoromethylpropenes was reported in 2014 [382]. A range of derivatives were prepared via an initial Grignard reaction followed by a Peterson elimination in a fully continuous process that made use of both an in-line extraction and solvent switching (Table 10).
Reaction times were shorter and the yields obtained were markedly better than analogous batch procedures reported by the same group later in the same year [383]. The crude product from the Grignard reaction was hydrolysed in-line using downstream inlets of aqueous acid followed by a 9:1 hexane/chloroform mixture which aided extraction via a liquid-liquid membrane-based continuous separator (Figure 10) [52]. 
Table 10: Synthesis of 3,3,3-trifluoromethylpropenes by continuous Grignard reaction.

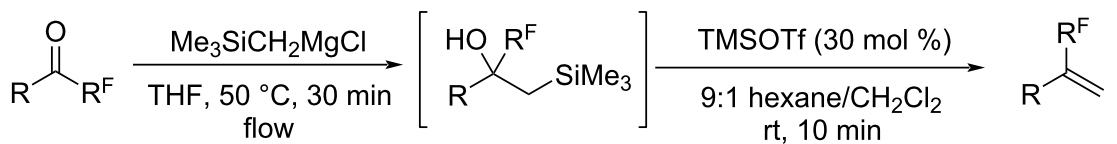

$$
\begin{aligned}
& \text { flow }
\end{aligned}
$$

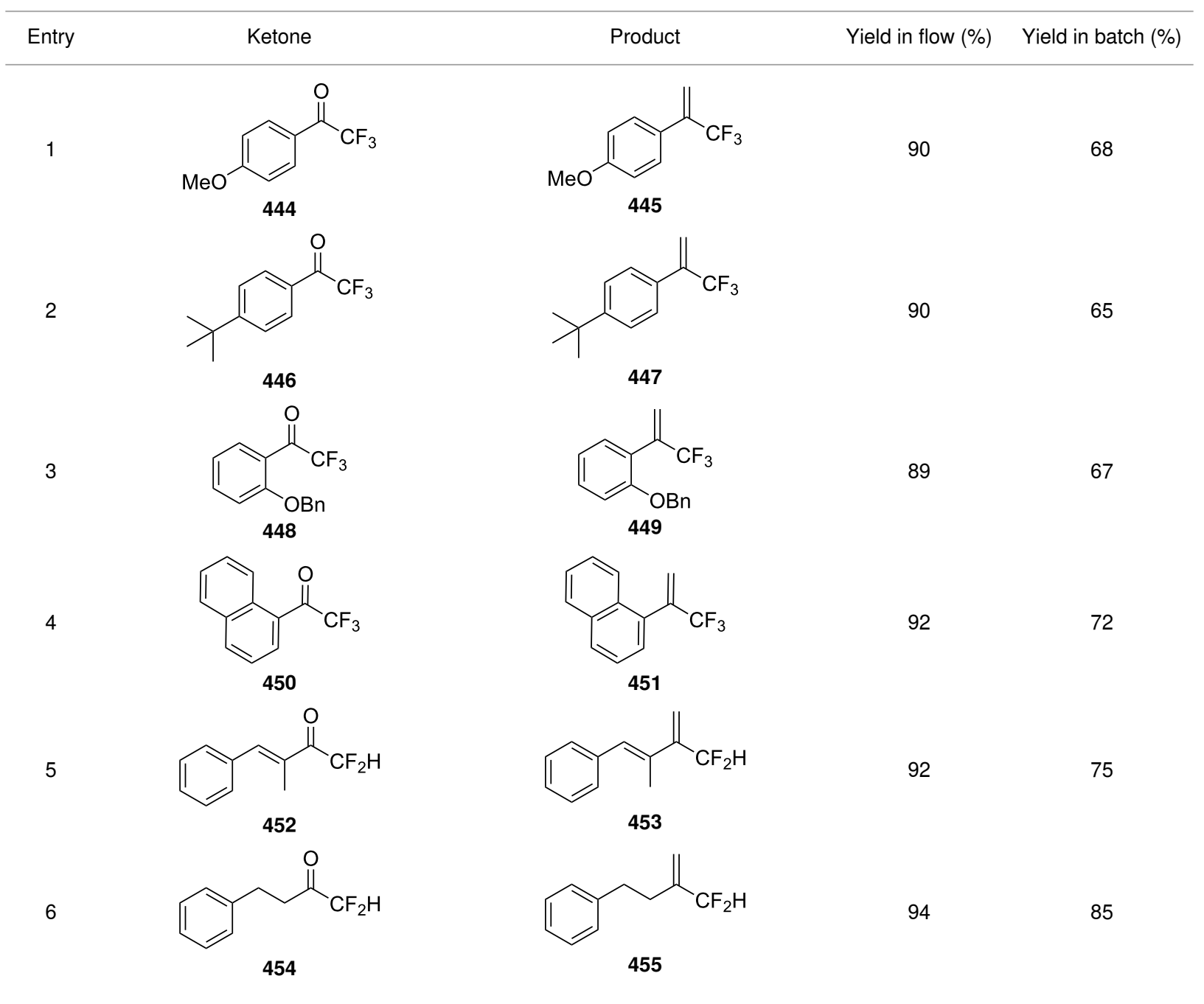
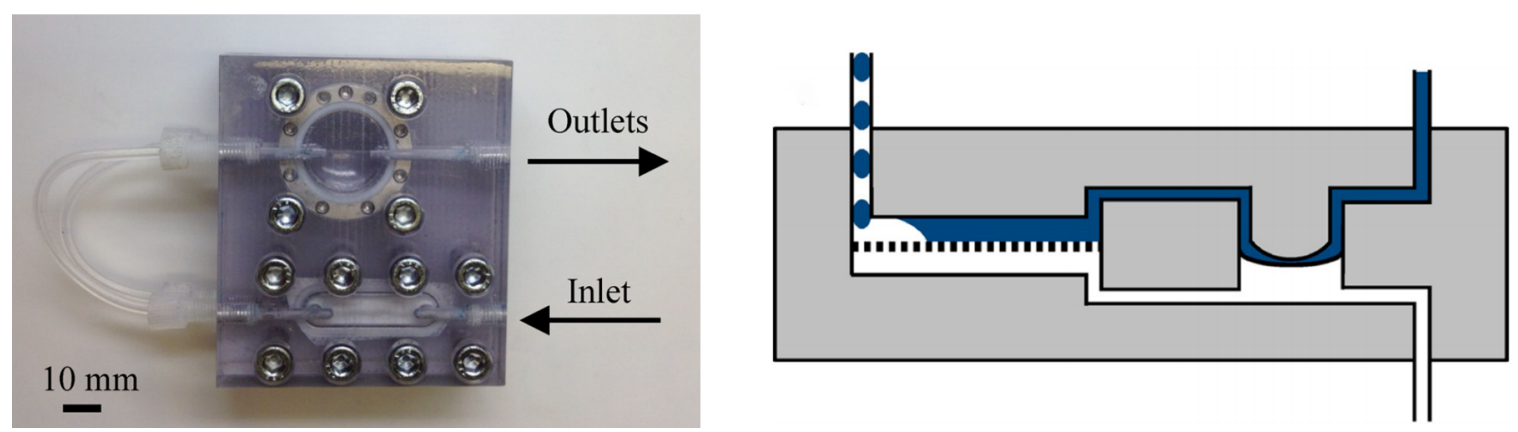

Figure 10: Membrane-based, liquid-liquid separator with integrated pressure control [52]. Adapted with permission from [52]. Copyright 2013 American Chemical Society. 
Many other multistep sequences that make use of Grignard reactions in flow can be found in the literature; excellent examples are found in the preparation of amitriptyline [384], (E/Z)-tamoxifen [212], and 2-aminoadamantane-2-carboxylic acid $[385,386]$.

In 2017, Gupton et al. developed a continuous-flow preparation for the intermediate of fluconazole (459), a potent antifungal, anti-HIV, and anticancer agent (Scheme 103) [387].

The flow apparatus consists of two steps: Grignard preparation and carbonyl addition. Initially, the two steps were optimised singularly and then telescoped. An excess of $\mathrm{iPrMgBr} \cdot \mathrm{LiCl}$ (1.8 equiv) was used to form the desired organomagnesium reagent from the 1-bromo-2,4-difluorobenzene (456). An excess of Grignard reagent was also employed in the second step to increase the conversion. After the flow systems, the authors realised the presence of a continuous stirring tanks reactor (CSTR) before the quenching with the ketone intermediate $\mathbf{4 5 7}$ (longer residence time) increased the yield of $\mathbf{4 5 8}$ (74\% vs $87 \%$ ). Due to the long reaction times of the last step, the synthesis of fluconazole (459) was finished using a batch step. For the specific Grignard addition into the carbonyl, the volume-time output (VTO) was calculated to be $8.962 \times 10^{-7} \mathrm{~m}^{3} \mathrm{~h} \mathrm{~kg}^{-1}$, which is in accordance with the aims of process chemistry $(\mathrm{VTO}<1)$.

Utilising Grignard reagents as strong bases to effect transformations in flow has also been shown with the Bodroux amide formation in 2012 [388] and continuous-flow Grignard addition to nitriles, acting as carbonyl surrogates, being reported in the following year [389]. Recently, amide formation was achieved using isocyanates as electrophiles in a copper-catalysed flow reaction. The flow system developed by Kerr, Leach and their co-workers employed stoichiometric amounts of reagents yielding amides in moderate to excellent yields [390]. In 2019, Wang, Castle and co-workers optimised the addition of Grignard reagents to benzoyl chlorides (Scheme 104) [391].

The reaction is extremely exothermic due to the combination of two strong electrophile/nucleophile being mixed. Also in this example, flow chemistry facilitates optimal mixing and heat transfer allowing a high selectively and good yield of the ketones (43-85\%). Interestingly, automation was incorporated into the reactor allowing several benzoyl chlorides to be screened sequentially using a 5-ways valve placed before the T-mixer. The system proved to be more efficient than the batch method ( $85 \%$ vs $34 \%$ ) and it could be employed for on-demand preparation of substrates enabling high throughputs (e.g., $3.16 \mathrm{~g} \mathrm{~h}^{-1}$ for the ketoprofen intermediate 462).

When moving to larger scales involving highly exothermic transformation such as Grignard additions, it is often problematic to conduct such processes in batch as safety concerns are greatly increased. The feasibility to scale up Grignard reactions in flow was demonstrated in a full-scale heterogeneous Grignard alkylation of a pharmaceutical intermediate $\mathbf{4 6 6}$ by Kiil et al. in 2013 (Scheme 105, Table 11) [392]. Due to solubility issues of $\mathbf{4 6 5}$, the design was based around a continuous stirred tank reactor (CSTR) which fed the solid $\mathbf{4 6 5}$ via a screw feeder and the Grignard solution, allylmagnesium chloride (464), via a pump. The reactor outlet was encased in a filter cartridge to

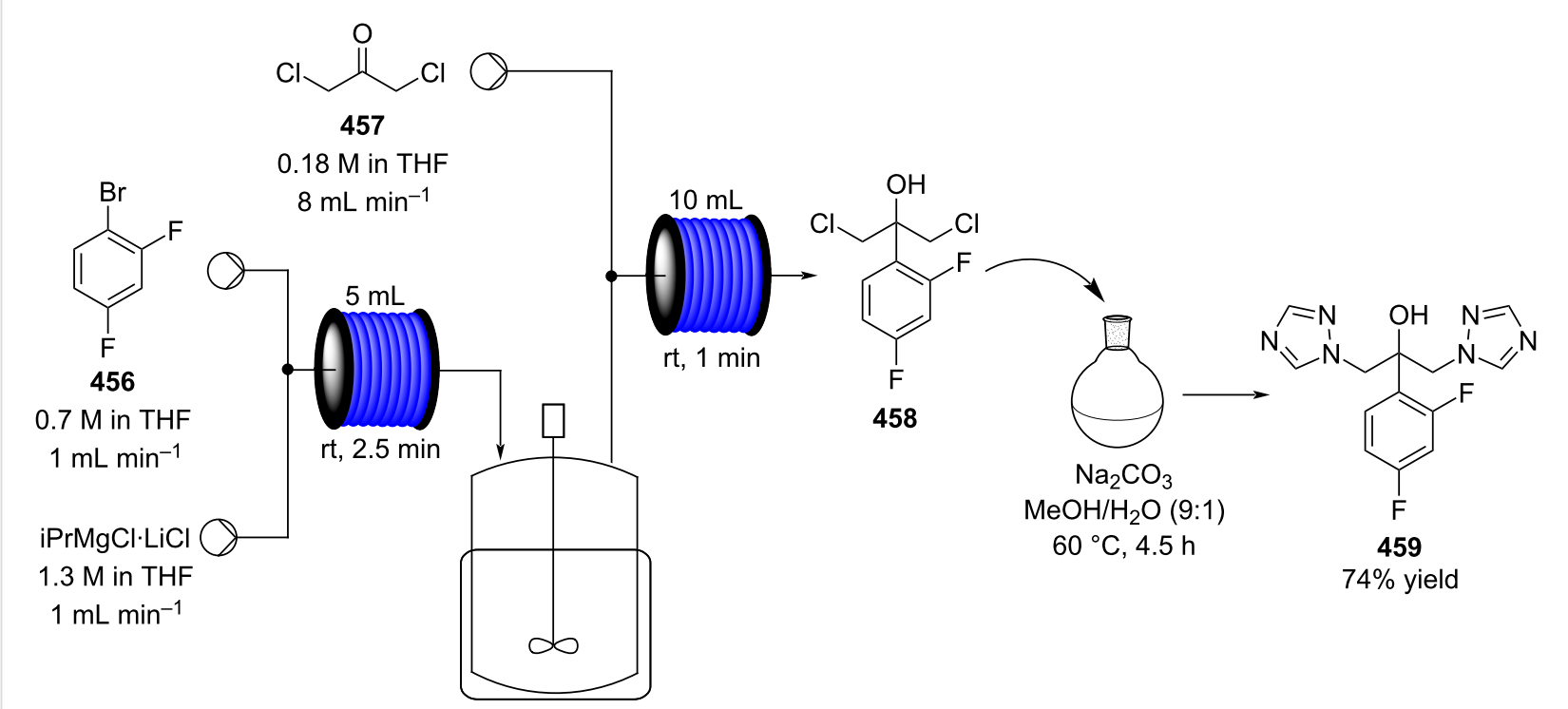




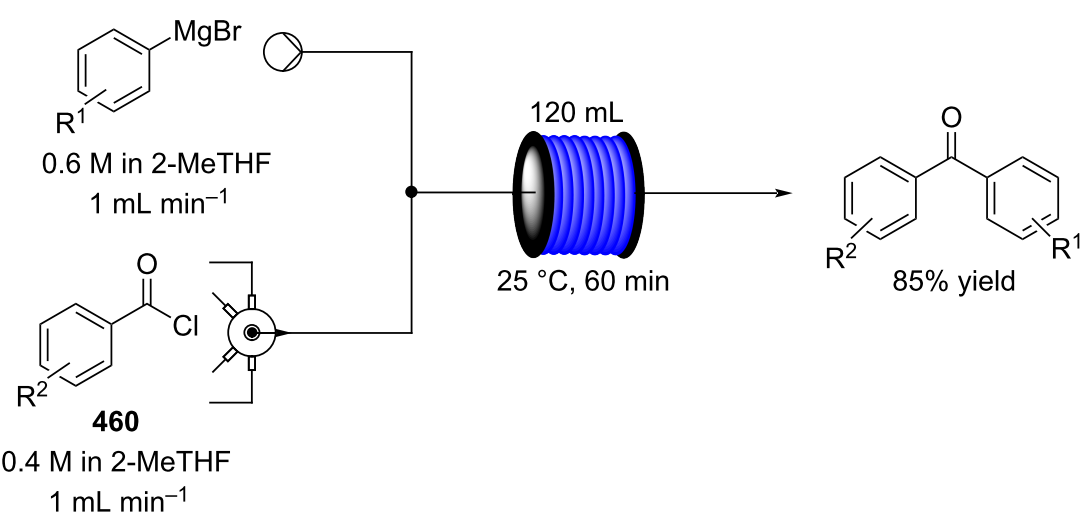<smiles>CC(C)(C)C(C)(C)C</smiles>

$43 \%$ yield<smiles>CC(C#N)c1cccc(C(=O)c2ccccc2)c1</smiles>

462

$56 \%$ yield

throughput $=3.16 \mathrm{~g} \mathrm{~h}^{-1}$<smiles>O=C(c1cccs1)c1ccccc1F</smiles>

463

$73 \%$ yield

Scheme 104: Continuous-flow synthesis of ketones starting from benzoyl chlorides.

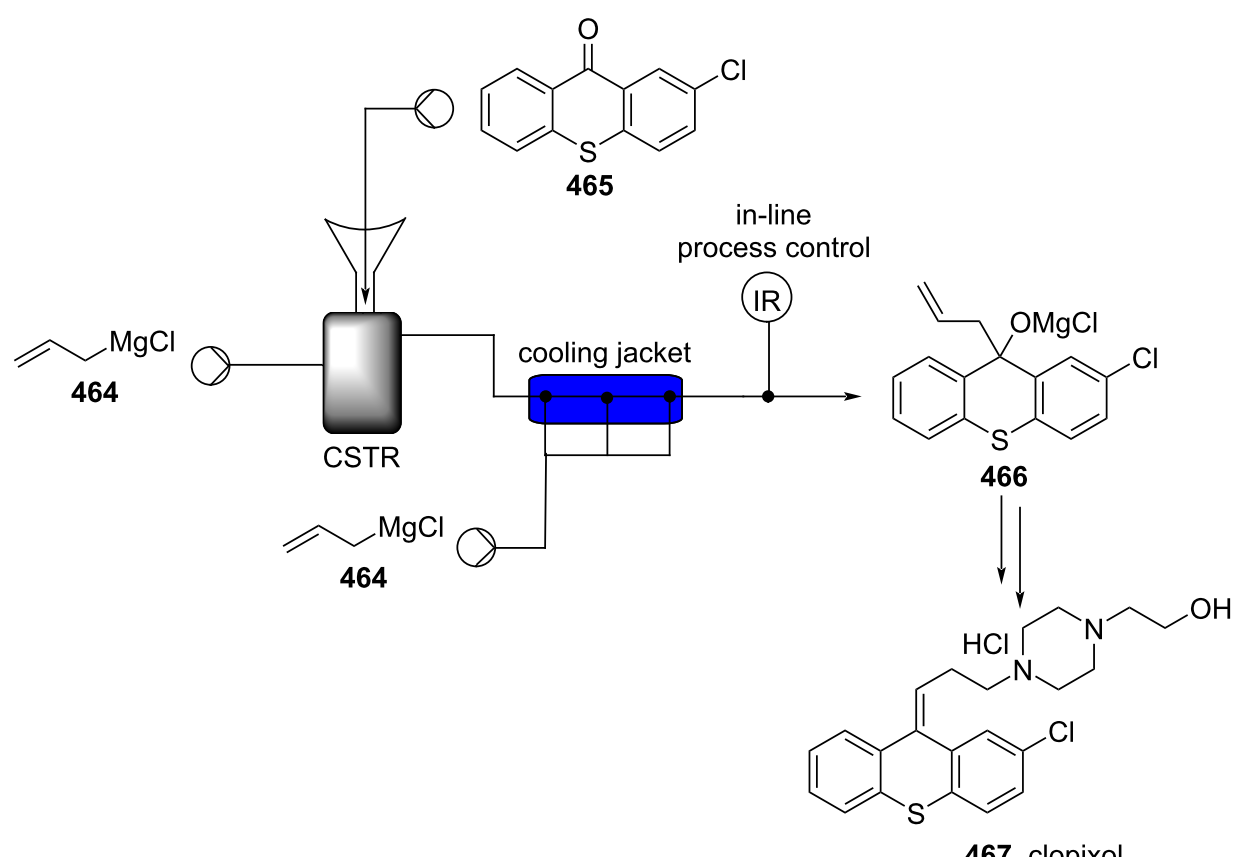

467, clopixol

Scheme 105: A Grignard alkylation combining CSTR and PFR technologies with in-line infrared reaction monitoring.

avoid further transfer of insoluble material into the secondary plug flow reactor (PFR).

Hydrolysis of the Grignard adduct $\mathbf{4 6 6}$ followed by further steps eventually produced clopixol (467), an antipsychotic drug.
Switching from a batch to flow regime afforded numerous advantages; a lower active volume, smaller setup size, improved yield and increased purity of product, a reduction in solvent consumption and favourable cleaning requirements. The incorporation of on-line infrared monitoring allowed for facile 
Table 11: Batch vs flow process comparison for the Grignard alkylation of 465.

\begin{tabular}{|c|c|c|c|}
\hline Parameter & $\begin{array}{l}\text { Full-scale } \\
\text { (semi-batch) }\end{array}$ & $\begin{array}{l}\text { Laboratory } \\
\text { (continuous) }\end{array}$ & $\begin{array}{c}\text { Full-scale } \\
\text { (continuous) }\end{array}$ \\
\hline active volume & $1600 \mathrm{~L}$ & $250 \mathrm{~mL}$ & $8 \mathrm{~L}$ \\
\hline total size & $\approx 10 \mathrm{~m}^{2}$ & $2 \mathrm{~m}^{2}$ & $1.45 \mathrm{~m}^{2}$ \\
\hline production time (h) & 4 & 900 & $<50$ \\
\hline yield (\%) & $>95$ & $>96$ & 95-99 \\
\hline purity (\%) & $>95$ & $>99$ & $96.5-99.8$ \\
\hline $\begin{array}{l}\text { solvent consumption } \\
(\mathrm{L} / \mathrm{kg} \text { of } 465)\end{array}$ & 5.8 & 2.3 & 3.8 \\
\hline cleaning & every batch & dedicated & dedicated \\
\hline
\end{tabular}

process control (process analytical technology, PAT), this is something that is well precedented and highly valuable for Grignard reactions [393-395].

In 2014, a mesoreactor scale-up was reported for the manufacture of ketones from the corresponding esters using a Grignard reagent [396]. Initial studies involved performing the reaction in a microchip reactor (internal volume $<1 \mathrm{~mL}$ ), an ART PR37 mesoreactor (internal volume $=13.6 \mathrm{~mL}$ ) was then used, resulting in a scalable procedure that could be viewed as an alternative to the Weinreb ketone synthesis (Scheme 106).

Continuous-flow processes can also be developed by modifying batch vessels. This approach presents several advantages, for instance it better tolerates solids, allows the use of higher concentration solutions, and it can make use of pre-existing plant equipment. At Eli Lilly batch vessels were explored for Grignard preparation, as magnesium solids may result in clogging of PFR's [397]. The CSTR system was continuously fed with aryl halide and the solution has a residence time in the vessel of roughly an hour. The so formed Grignard reagent was then removed and, to control the solid presence in the solution, passed through a pre-settling pipe and a Mg trap. The apparatus operated over 14 hours ( 2 working days) yielding the desired reagent 471 in $97 \%$ conversion, reducing the amount of $\mathrm{Mg}$ by $50 \%$ compared with the batch process (Scheme 107).

The newly formed organomagnesium halide was employed for the development of the continuous synthesis of $\mathbf{4 7 2}$, an intermediate of edivoxetine hydrochloride (473), an antidepressant agent (Scheme 108).

Interestingly, the authors conducted a comparison of the synthesis of compound 472 using the described CSTR Grignard process and employing a PFR-optimised lithiation. The Grignard process was selected as the most suitable method of preparation for their needs, as such a $2 \mathrm{~L}$ scale-up was carried out and after 32 hours (5 working days) roughly $4 \mathrm{~kg}$ of $\mathbf{4 7 2}$ was obtained in a yield of $82 \%$. Crystallisation of the crude product afforded the desired intermediate in $>99.8 \%$ purity with an overall yield of $78 \%$; an improvement in the yield of over $10 \%$.

Combining Grignard reactions with various gases in flow has also been achieved furnishing ketones [398], carboxylic acids [258] and phenols [399,400]. As early as 2011, the Ley group reported the use of a tube-in-tube reactor to effect the conver-

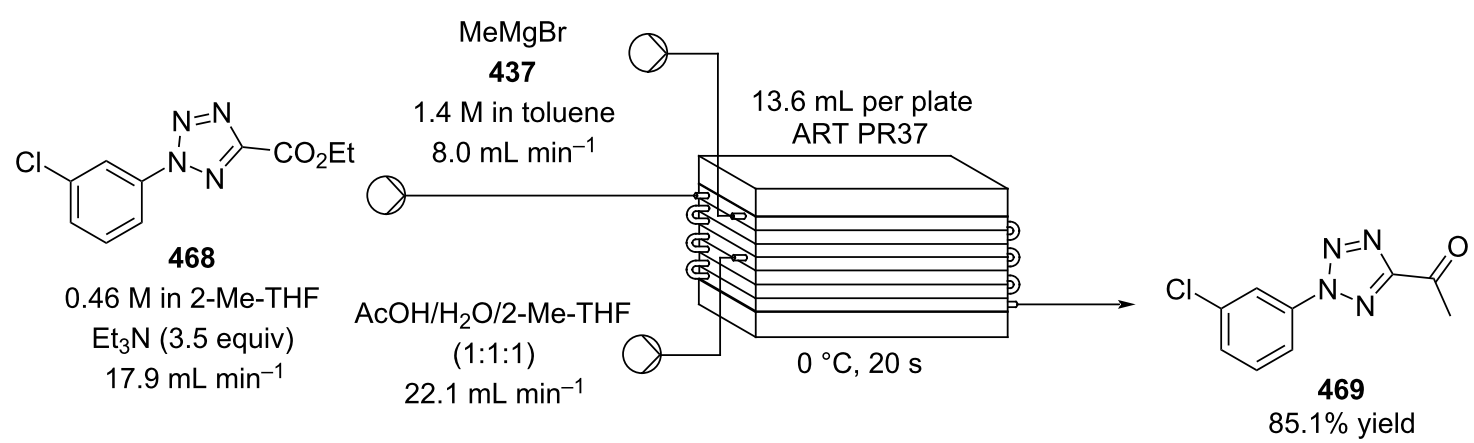

Scheme 106: Continuous-flow preparation of $\mathbf{4 6 9}$ from Grignard addition of methylmagnesium bromide. 


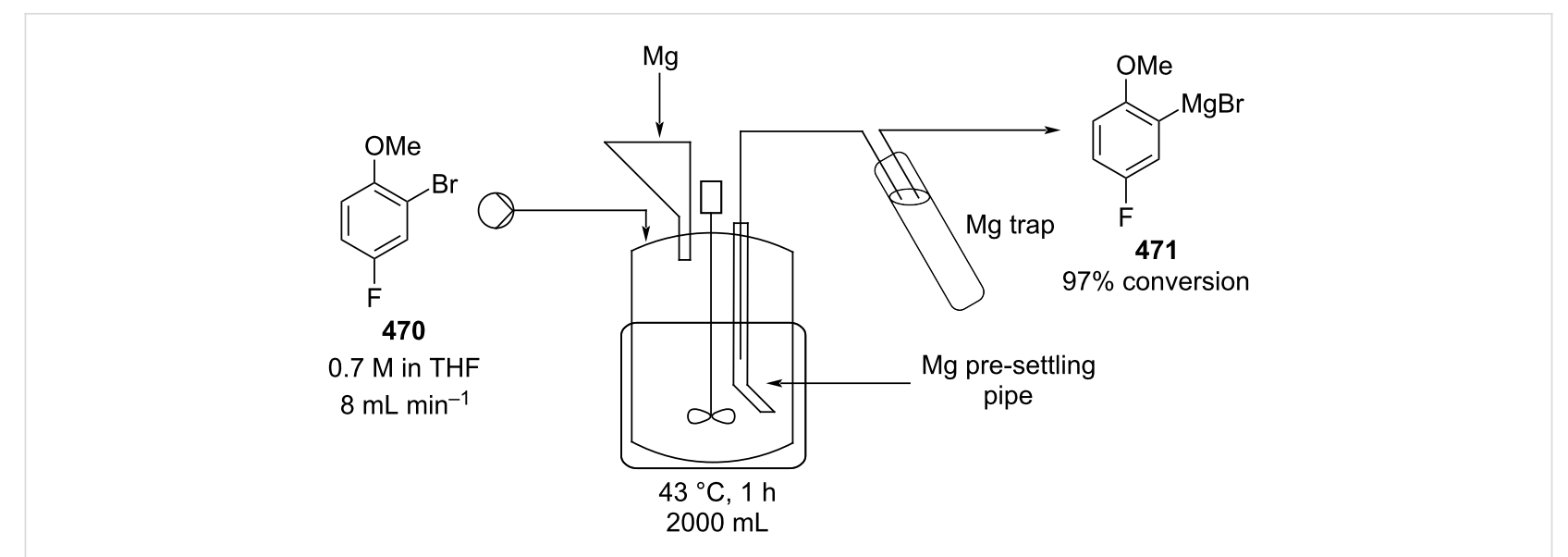

Scheme 107: Continuous-flow synthesis of Grignard reagents 471.

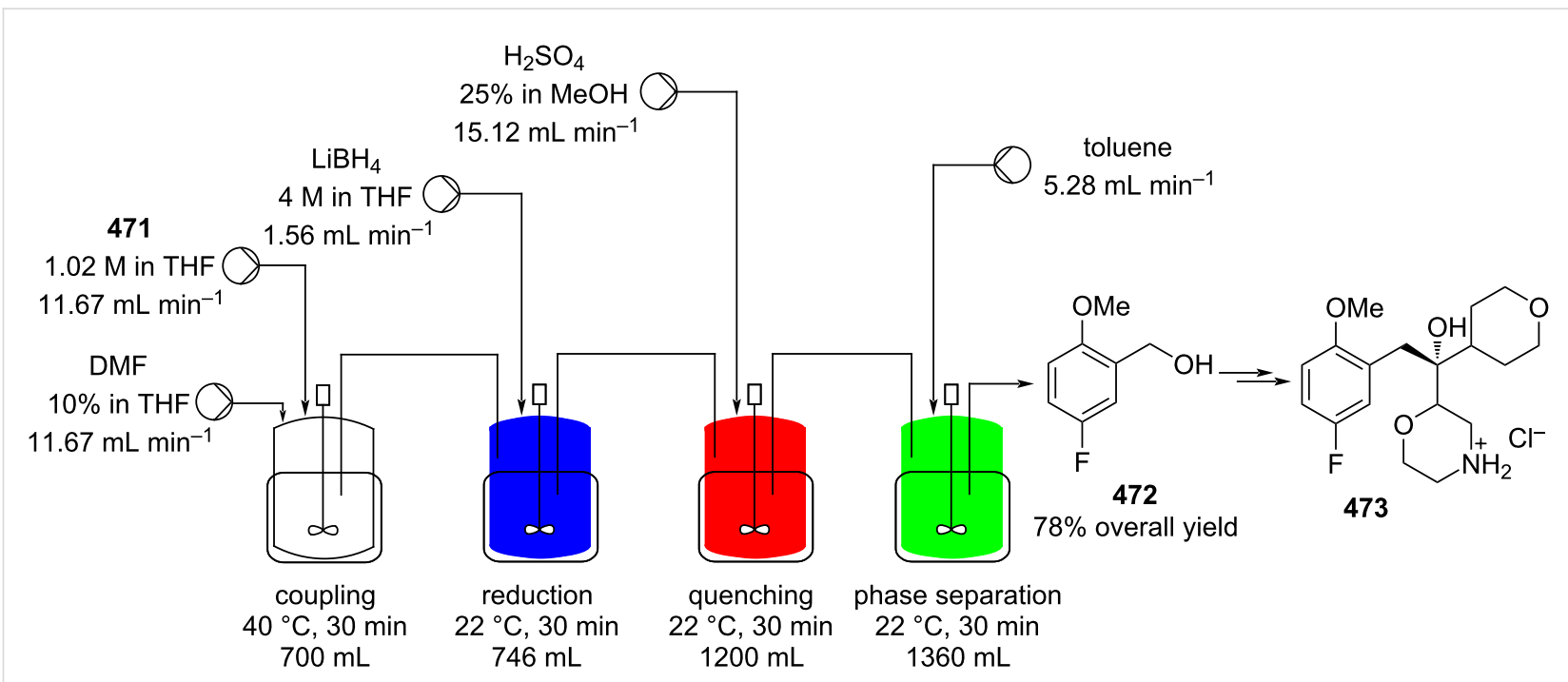

Scheme 108: Preparation of the Grignard reagent 471 using CSTR and the continuous process for synthesis of the edivoxetine's intermediate 472.

sion of a collection of aromatic Grignard reagents to their corresponding carboxylic acids in good to excellent yields (Scheme 109) [258].

A polymer-supported sulfonic acid column allowed in-line scavenging of the magnesium salts upon exiting the main reactor while simultaneously protonating the carboxylate product. A "catch-and-release" strategy was then adopted to further purify the product by using a polymer-supported ammonium hydroxide column.

In 2014 , the use of acetylene gas for the generation of an ethynyl-Grignard reagent and subsequent synthesis of the propargylic alcohols 474-479 was reported in flow (Scheme 110) [401]. The initial formation of the Grignard reagent from ethylmagnesium bromide and acetylene gas was conducted in a falling film microreactor (FFMR) with the output being telescoped into a second reactor along with a range of ketones. After acidification using ammonium chloride, the corresponding propargylic alcohols could be isolated. The methodology was applied to eighteen different substrates achieving mostly high yields (one $58 \%$ and all others $>88 \%$ ) and high selectivity (all $>92 \%$ ). In a similar way a Chinese patent application has been made for the process to be performed within a microstructured reactor [402].

Recently, Grignard reagents have been exploited in enantioselective arylation of aldehydes using a silica-supported catalyst ( $\mathrm{H}_{8}$-BINOL-derivative) [403]. The grafted catalyst was placed in a disposable pipette $(6 \times 100 \mathrm{~mm})$ connected to syringe pumps. At first, a solution of the Grignard and titanium isopropoxide in DCM $(0.1 \mathrm{M})$ was eluted to form the activated 


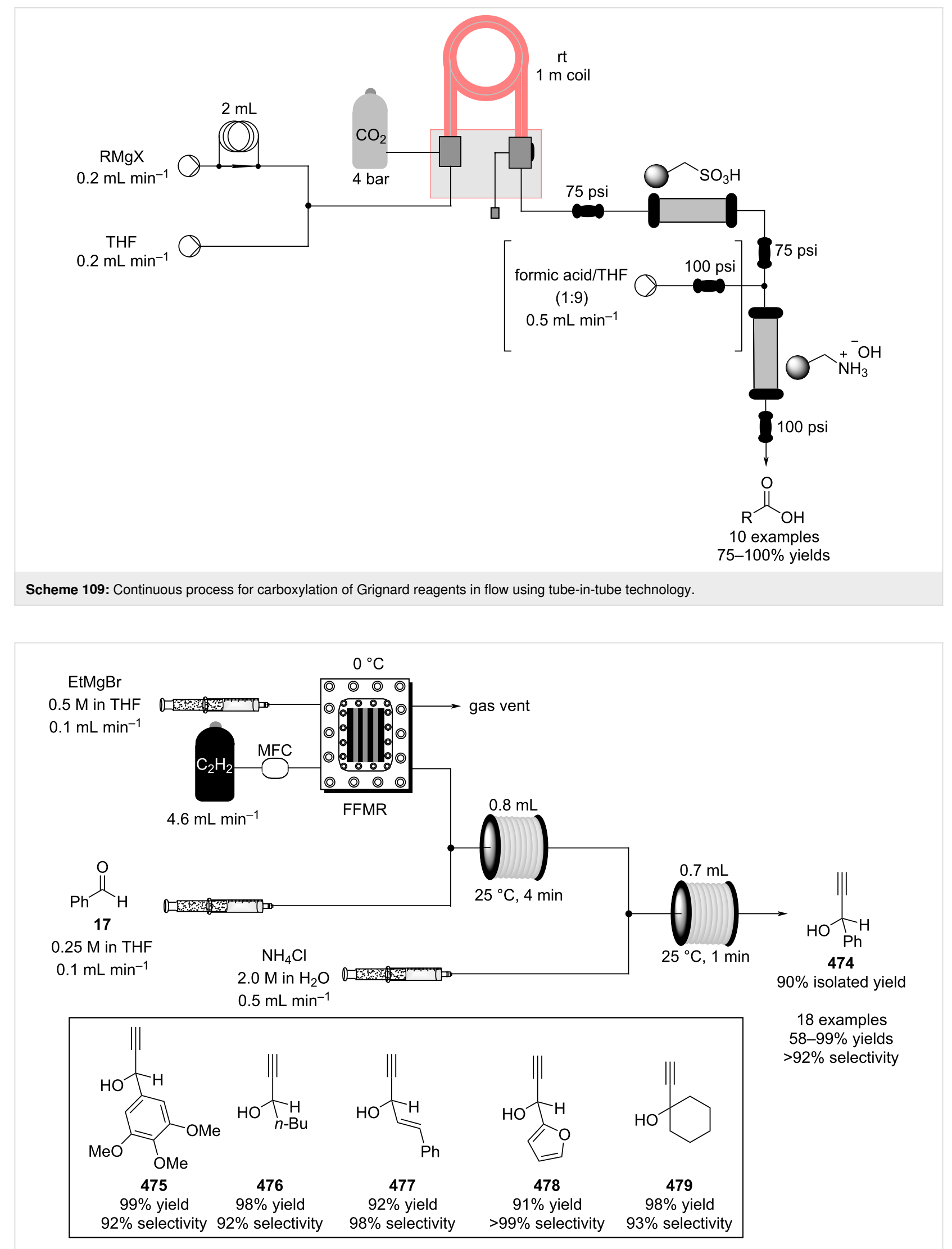

Scheme 110: Continuous synthesis of propargylic alcohols via ethynyl-Grignard reagent. 
Ti catalyst. Once formed, a solution of the aldehyde in DCM $(0.2 \mathrm{M})$ was introduced at a set flow rate that maintained the molar ratio between the reagents at 2 equivalents. After 30 minutes residence time, the solution was quenched. Several substrates were screened furnishing moderate to good conversions (40-80\%) and good to excellent enantioselectivites (83-95\%). Of particular importance is that the column could be repeatedly used for several experiments revealing the catalyst to be stable under the flow processing conditions (Scheme 111).

\section{Isomerisations and rearrangements}

Terpene-like products are key materials in medicinal and F\&F chemistry, where several total syntheses have been developed [376]. Isomerisation and rearrangement reactions are commonly employed during several stages of their production (Scheme 112) [404]. For instance, the synthesis of $\beta$-ionone (481) is made possible via an acid-catalysed rearrangement of $\Psi$-ionone (80) [405]. Similarly, galbascone (484) may be prepared by an acid-catalysed rearrangement of the dehydration product of dehydrolinalool (454) [406]. Acid-catalysed rearrangement have also been employed for the synthesis of musk acetate (487) [407]. Pyrolysis of $\alpha$-pinene to $o$-cimene (488) has also found great interest in preparation of $F \& F$ intermediates [408].

The selectivity and efficiency of many isomerisation and rearrangement reactions are highly dependent on the reaction temperature or irradiation wavelength and are therefore well suited to flow processing (improved contact surface areas resulting in better mass and heat transfer). This has been exemplified for the photo-oxidation of a range of fragrance-type substrates $[312,313,315,409]$. More recently, a continuous-flow system for catalytic alkene isomerization using visible light was reported by Rueping et al., for which the conversion of trans- (489) to cis-stilbene (489) was studied (Scheme 113) [410].

A batch process was initially developed involving the use of an $\left[\operatorname{Ir}(\mathrm{ppy})_{2}(\mathrm{bpy})\right]\left(\mathrm{PF}_{6}\right)$ photoredox catalyst with the ionic liquid, $[\mathrm{Bmim}] \mathrm{BF}_{4}$, which was eventually translated into a flow system with recycling of the easily spreadable photoredox catalyst system. Despite the relatively low flow rates investigated $\left(<20 \mathrm{~mL} \mathrm{~h}^{-1}\right)$, quantitative conversions were achieved without loss of the valuable iridium catalyst. In a related set of examples an iridium catalysed alkene isomerisation has also been shown to be possible in flow by using an immobilised version of Felkin's catalyst [126,411]. Trans-to-cis alkene photoisomerisation was also performed in flow on a ${ }^{18} \mathrm{~F}$-labelled $\mathrm{cis}$ cyclooctene employed as dienophile for Diels-Alder (DA) reaction [412].

Claisen rearrangements in flow have been studied extensively [413-419] and in 2011 a report by scientists at Eli Lilly \& Co. looked at comparing the thermal ortho-Claisen rearrangement of the allyl aryl ether, 490, an important early phase intermediate, in flow to batch (Table 12) [417]. Significant advantages were associated with the flow protocol, stemming from the ability to use higher concentrations (even solvent-free conditions), pressures and temperatures in a safe manner [419]. As a result, a much safer and highly reproducible system was achieved which was not easier to perform at higher temperatures, but also was associated with less problematic workup. Interestingly, in this area continuous-flow microwave reactors have also been developed for high temperature and pressure Claisen rearrangements $[413,418,420]$.

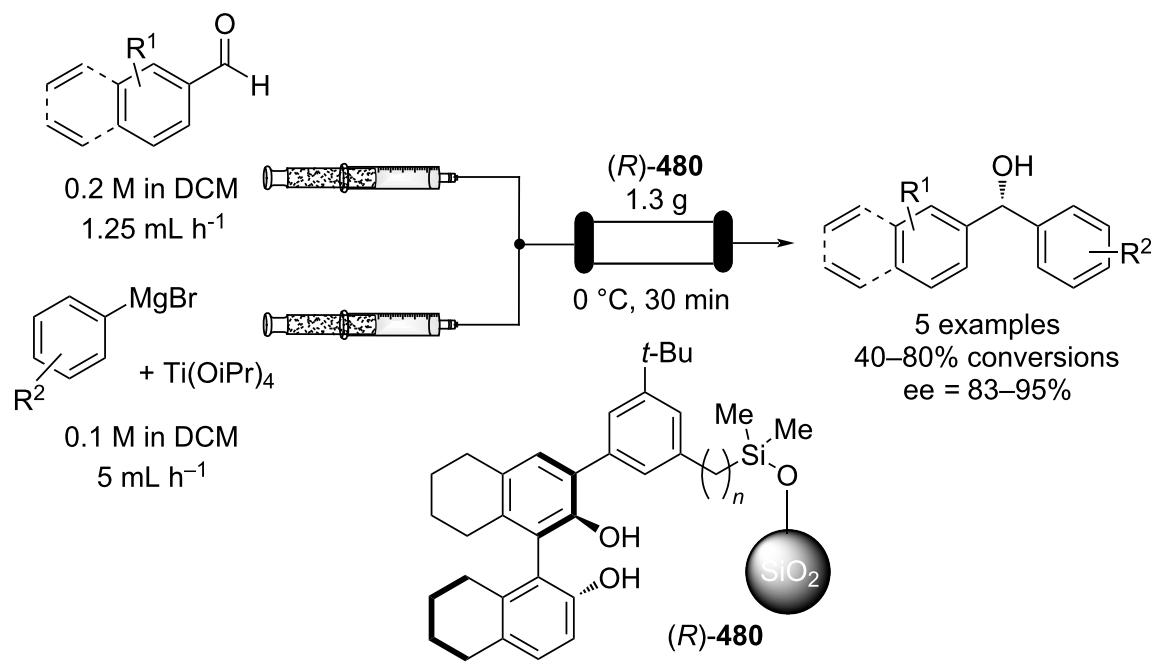

Scheme 111: Silica-supported catalysed enantioselective arylation of aldehydes using Grignard reagents in flow conditions. 
<smiles>CC(=O)C=CC=C(C)CCC=C(C)C</smiles>

80
1. $\mathrm{H}_{2} \mathrm{SO}_{4}$

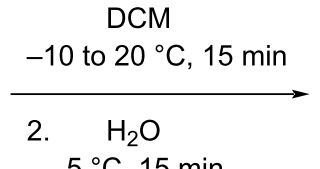

$5{ }^{\circ} \mathrm{C}, 15 \mathrm{~min}$<smiles>CC(=O)/C=C/C1=C(C)CCCC1(C)C</smiles>

481

$90 \%$
$+$<smiles>CC(=O)/C=C/C1C(C)=CCCC1(C)C</smiles>

$1 \%$<smiles>C=CCC#CC(=C)CCC=C(C)C</smiles>

483

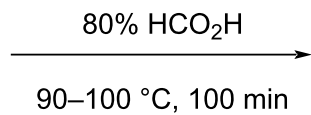

$90-100{ }^{\circ} \mathrm{C}, 100 \mathrm{~min}$<smiles>C=CCCC(=O)C1=CCCC(C)(C)C1</smiles>

484
$+$<smiles>C=CCCC(=O)C1=CC(C)(C)CCC1</smiles>

485

484/485 $=2: 3$

$75 \%$ yield

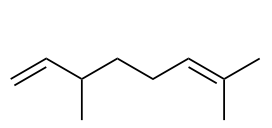

486

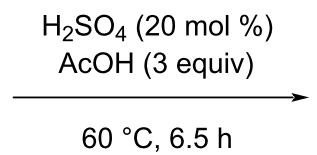

$60{ }^{\circ} \mathrm{C}, 6.5 \mathrm{~h}$

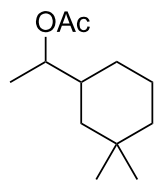

487

$45 \%$

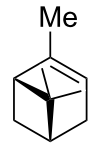

213

\section{$650^{\circ} \mathrm{C}, 10 \mathrm{~min}$}

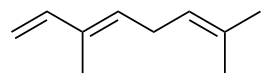

488

$46.5 \%$

Scheme 112: Acid-catalysed rearrangement of citral and dehydrolinalool derivatives.

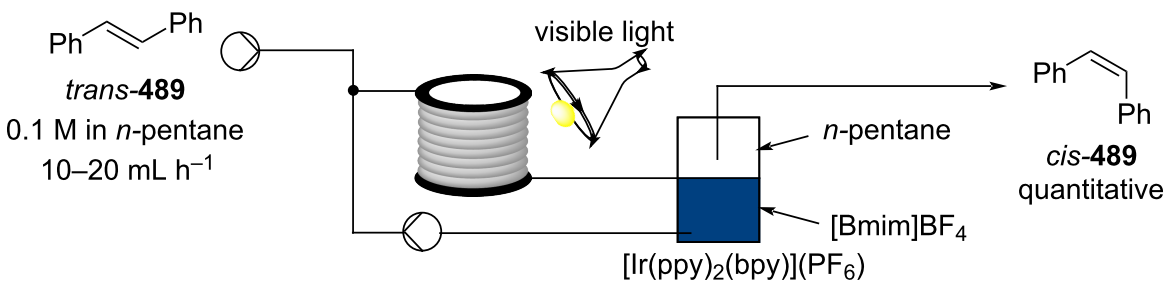

Scheme 113: Continuous stilbene isomerisation with continuous recycling of photoredox catalyst.

In 2015, a continuous-flow method for the preparation of $o$-cimene (488) from thermoisomeric $\alpha$-pinene $(\mathbf{2 1 3})$ in the liquid phase was patented by Jiangxi Jiayuan Fragrance Co Ltd. [421]. One year later, Ley and co-workers developed a continuous-flow 2-step telescoped solvent-free synthesis of 2-propylphenol (494), a smoky, and phenolic fragrance compound, starting from allyl phenyl ether (492, Scheme 114) [422].
The flow approach employed a Phoenix reactor (ThalesNano ${ }^{\circledR}$ ) as a high temperature operating device. In this example, a Claisen rearrangement was performed in 1 minute at $320^{\circ} \mathrm{C}$ and $100 \mathrm{bar}$. The reaction was also easily scale-up from $60 \mathrm{~g} \mathrm{~h}^{-1}$ to $480 \mathrm{~g} \mathrm{~h}^{-1}$ by only changing the reactor heated volume (from 1 to $8 \mathrm{~mL}$ ). As different flow rates were required to perform the two reaction steps, the intermediate $\mathbf{4 9 3}$ was collected in a 
Table 12: Batch vs flow for the thermal Claisen rearrangement of an ally aryl ether $\mathbf{4 9 0 .}$

\begin{tabular}{|c|c|c|}
\hline & Batch process & Flow process \\
\hline solvent & diphenyl ether & $N$-methylpyrrolidinone \\
\hline concentration & 33 wt \% & 50 wt \% \\
\hline temperature & $220^{\circ} \mathrm{C}$ & $230^{\circ} \mathrm{C}$ \\
\hline solvent bp & $259^{\circ} \mathrm{C}$ & $202-204^{\circ} \mathrm{C}$ \\
\hline operating pressure & 1 bar & 15 bar \\
\hline reaction time & $5 \mathrm{~h}$ & $4 \mathrm{~h}$ \\
\hline workup & $\begin{array}{l}\text { crystallisation on cooling (potential to } \\
\text { freeze solvent below } 27^{\circ} \mathrm{C} \text { ) }\end{array}$ & aqueous drown out and extraction \\
\hline safety & potential for runaway reaction & $\begin{array}{l}\text { improved heat transfer provides better temperature control; } \\
\text { contained system; potential to safely run at higher } \\
\text { concentrations }\end{array}$ \\
\hline manufacturability & $\begin{array}{l}220^{\circ} \mathrm{C} \text { not easily reached by typical } \\
\text { batch reactor heat transfer systems }\end{array}$ & $230^{\circ} \mathrm{C}$ easily reached in ovens \\
\hline robustness & $\begin{array}{l}\text { reaction temperature variations } \\
\text { leading to variation in yields and } \\
\text { purity }\end{array}$ & highly reproducible \\
\hline
\end{tabular}

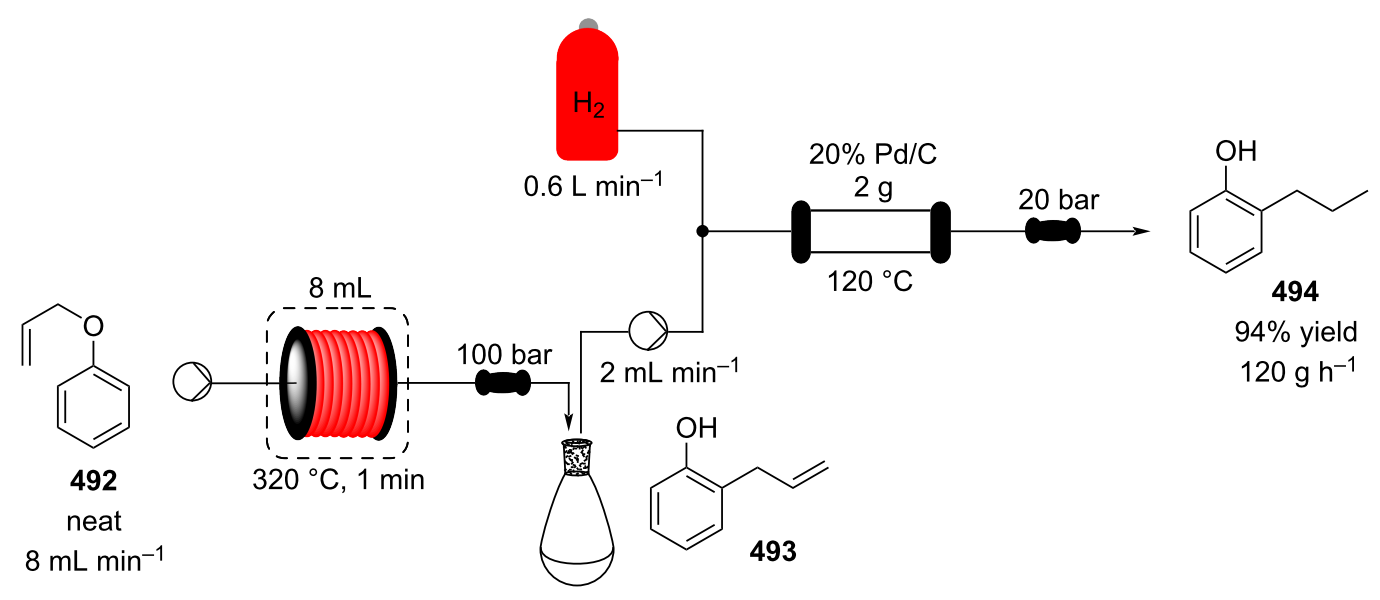

Scheme 114: Continuous-flow synthesis of compound 494 as developed by Ley et al.

reservoir, ready to be streamed in the secondary hydrogenator. The final step involves a $3 \mathrm{~mL}$ column reactor packed with $20 \%$ $\mathrm{Pd} / \mathrm{C}$ heated at $120{ }^{\circ} \mathrm{C}$. The optimised setup enabled the preparation of the desired material 494 in $94 \%$ yield with a promising throughput of $120 \mathrm{~g} \mathrm{~h}^{-1}$.

Isomerization and rearrangements in flow chemistry are relatively underexplored and is an area of active research currently.

\section{Cycloaddition reactions}

Cycloaddition reactions represent a powerful tool for the construction of rings, especially fused systems. It can also provide precursors to linear molecules through ring opening via ozonolysis and metathasis processes. Diels-Alder (DA) reactions have been extensively exploited by the F\&F industry and a rich body of work has been published in the literature [423]. DA synthesis of an Iso-E-Super intermediate $\mathbf{4 9 8}$ has been performed 
using aluminium trichloride as a Lewis acidic catalyst [424]. A $\delta$-damascone ( $\delta$-412) precursor is also prepared in this way as a further important example (Scheme 115) [425].

Although widely explored, the number of industrial applications is still low compared to other chemical transformations, mainly due to safety concerns originating from the inherent reactivity of dienes and dienophiles with regard to competing runaway reactions like polymerisation. Hence, flow chemistry and its advantage in respect to exacting control over reaction conditions has proved useful, reducing the likelihood of exothermic side reactions.

In 2015, a multistep flow synthesis of a spirocyclic compound $\mathbf{5 0 5}$, which is a potential fragrance compound, was achieved
[426]. It's precursor 504 can be synthesised via a DA dimerization of the intermediate diene $\mathbf{5 0 3}$ which was obtained via a Baylis-Hillman transformation and activated elimination of the hydroxy group. This process provided a robust way to generate product $\mathbf{5 0 4}$ in $89 \%$ purity, obtaining after 6.5 days $3.58 \mathrm{~kg}$ of product with a throughput of $23 \mathrm{~g} \mathrm{~h}^{-1}$ (Scheme 116).

Recently, continuous-flow DA cycloadditions have been performed on myrcene (506), which is an acyclic monoterpene widely used as a building block in the F\&F industry [427]. The authors wanted to prepare a new potential surfactant using myrcene (506) and acrylic acid (507). The DA adduct 508 obtained was scaled up from the batch to flow systems. The flow apparatus enables a reduction in reaction time $(10 \mathrm{~h}$ vs $30 \mathrm{~min})$<smiles>C=C(/C=C/C)CCC=C(C)C</smiles>

495
496

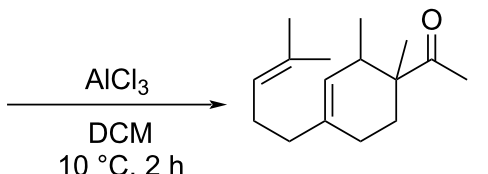

497
$10{ }^{\circ} \mathrm{C}, 2 \mathrm{~h}$<smiles>C/C=C\C=C/C</smiles>

499

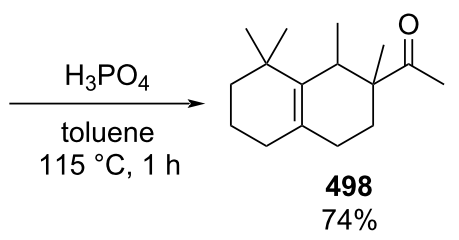

1.<smiles>CCCCC=C[O-]</smiles>
$\mathrm{EtMgBr}$ $0{ }^{\circ} \mathrm{C}->\mathrm{rt}$ rt, $30 \mathrm{~min}$

2. $\mathrm{TsOH}$ (cat.) reflux, Dean-Stark

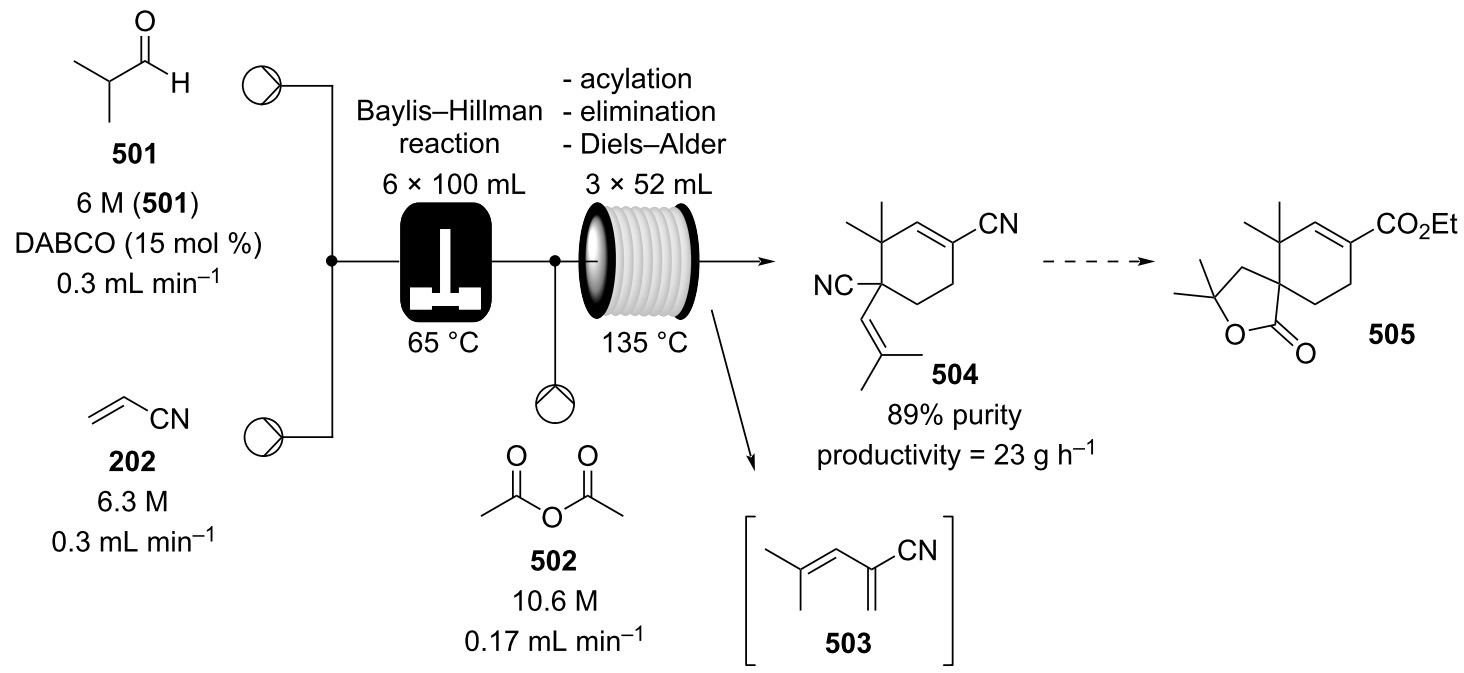


through an increase in temperature in the flow system $\left(110^{\circ} \mathrm{C}\right.$ vs $160{ }^{\circ} \mathrm{C}$ ), demonstrating a notable process intensification. Two different flow reactors of different sizes were employed in the scale-up: a $10 \mathrm{~mL}$ plug reactor (Vapourtec R-series), and a $100 \mathrm{~mL}$ plate reactor (Chemtrix Plantrix ${ }^{\circledR}$ MR260) (Scheme 117). In both case similar results were obtained (99\% vs $100 \%$ ), with an increase of 10-times the productivity to $2.79 \mathrm{~kg}$ of $\mathbf{5 0 8}$ per day $\left(\mathrm{STY}=1.11 \mathrm{~kg} \mathrm{~L}^{-1} \mathrm{~h}^{-1}\right)$.

DA reactions mostly involve the use of an $\alpha, \beta$-unsaturated ketone or aldehyde as the dienophile component. Lowering the energy of the LUMO of the dienophile via the incorporation of a Lewis acid is a commonly employed strategy in catalysing such reactions. While the use of salts such as aluminium(III) chloride may pose solubility issues in flow, numerous methods for the continuous processing of reaction slurries have been reported [44,428-431]. Alternative methods of catalysis have also been developed over the past few decades which offer feasible transposition to flow. Zeolite [432,433], heteropoly acids (HPA) [434] and other Lewis acid catalysis [435] have been exploited under flow conditions.

In addition, enantioselective organocatalytic DA reactions were first reported in 2000 by MacMillan et al. [436], following further discoveries [437], such catalysts have been incorporated into flow. Silica-supported imidazolidinones were used by Benaglia et al. in 2013 for stereoselective continuous-flow DA reactions of cyclopentadiene (510, Scheme 118) [438,439]. More recently, a monolith reactor containing polymer-based imidazolidinones has been developed by the same group [440]. An TADDOL-based organometallic version has also been attempted although this was less effective delivering poor ee $(6.5-25 \%)$ [441].

The advantages of conducting DA reactions in flow have been more widely demonstrated. In 2012 Abele et al. reported the DA reaction of (cyclohexa-1,5-dien-1-yloxy)trimethylsilane (513) with dienophiles $\alpha$-acetoxyacrylonitrile and acrylonitrile in flow (Scheme 119) [442]. Batch to flow comparisons revealed that overcoming thermokinetic issues associated with batch scale-ups was possible by adopting a flow approach. For the process involving acrylonitrile, access to a much larger temperature window $\left(<215{ }^{\circ} \mathrm{C}\right.$ compared to $\left.<90{ }^{\circ} \mathrm{C}\right)$ in flow allowed for accelerated reaction. A residence time of only 1 minute (cf. reaction time of 20 hours in batch at $90{ }^{\circ} \mathrm{C}$ ) using a simple stainless steel tube reactor design gave a productivity of $96 \mathrm{~g} \mathrm{~h}^{-1}$. Other DA reactions have similarly shown reductions in reaction time [443-446].

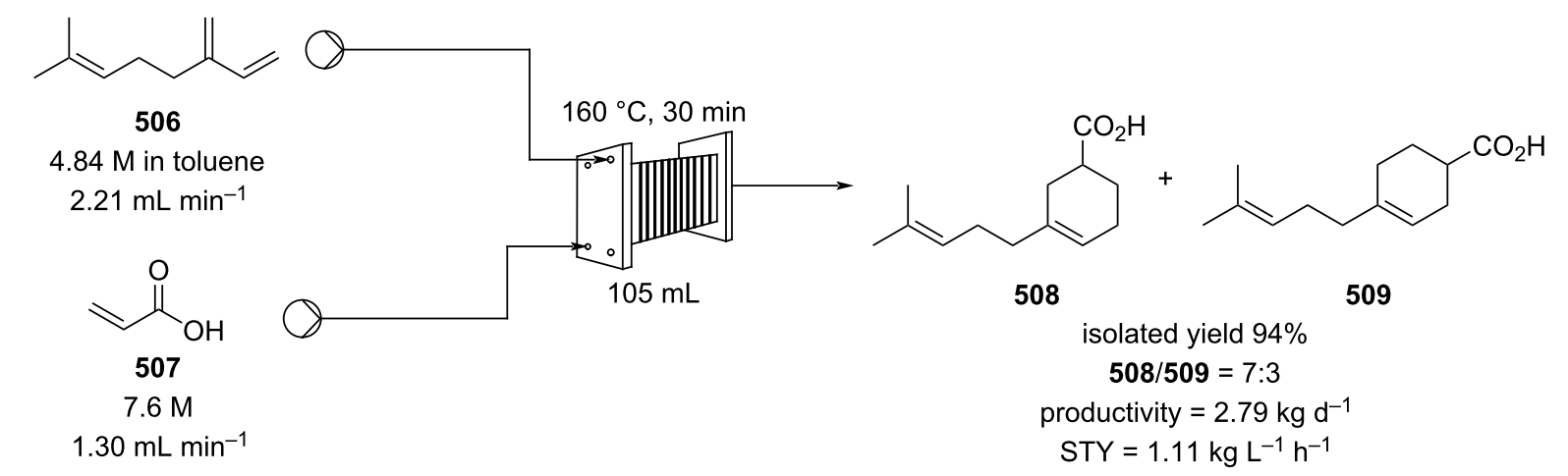

Scheme 117: Continuous-flow DA reaction developed in a plater flow reactor for the preparation of the adduct $\mathbf{5 0 8}$ from myrcene (506).

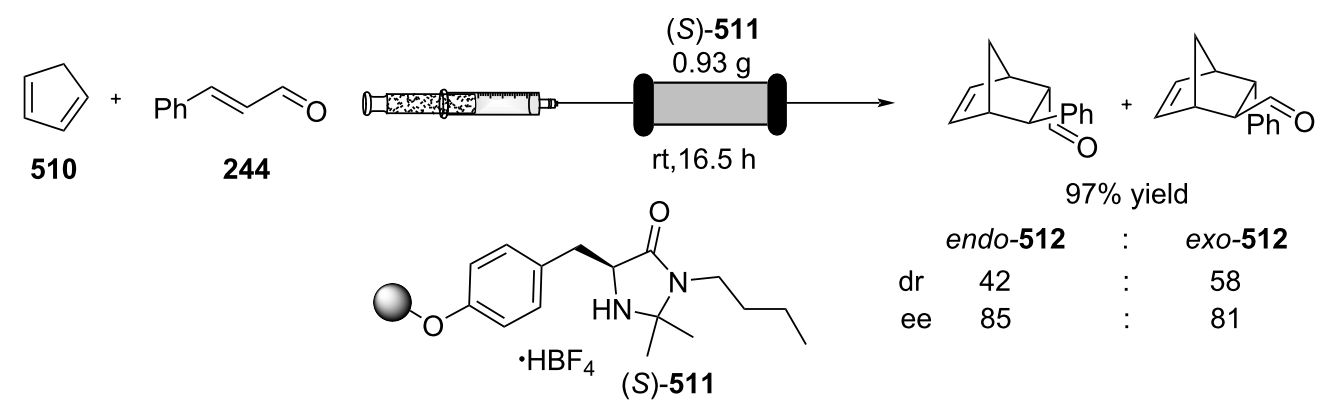




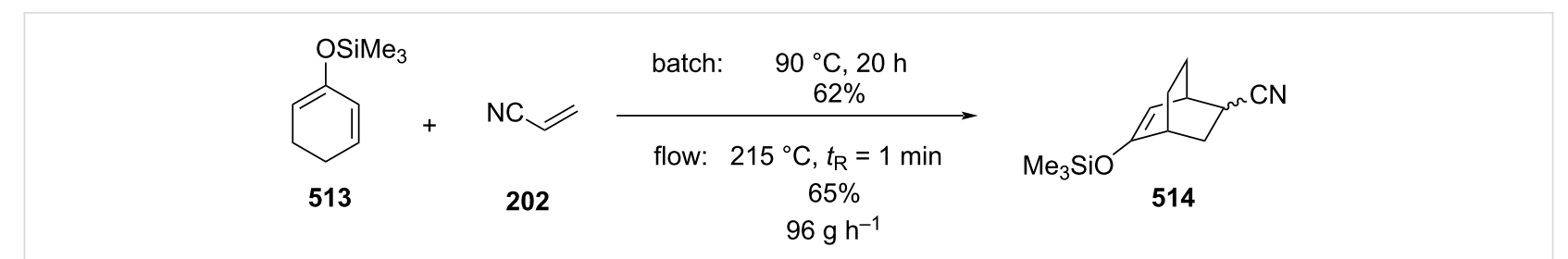

Scheme 119: Batch vs flow for the DA reaction of (cyclohexa-1,5-dien-1-yloxy)trimethylsilane (513) with acrylonitrile.

As DA reactions are characterised by a negative volume change, biphasic systems notably accellerate the reaction due to solvophobic effects. Karl and Löwe developed a flow system where this effect is exploited to enhance the reaction rates (Scheme 120) [447].

The apparatus consists of a segmented flow stream where core-shell droplets made of water (core) and the reaction mixture (shell) were directed into a horizontally placed coil. The droplets were formed utilising a engineered processing unit [448] and streamed by means of a fluorinated solvent (FC$\left.40^{\mathrm{TM}}\right)$. The DA reaction between cyclopentadiene (510) and methyl acrylate (515) was optmised. The authors pointed out the importance of the reactor positioning as gravity-induced mixing significantly improved the outcomes. The system was found to be more efficent than a slug flow one, allowing to perform the experiment at room temperature without the need for temperature and pressure control.

As discussed in previous sections, reactive intermediates can be preprared in situ and immediately utilised in consecutive steps of a integrated flow system. An example, of this was developed by Organ et al. where precursor $\mathbf{5 1 8}$ was used to form the benzyne intermediate, which then underwent a DA cycloaddition with cyclic dienes (furan, imidazole) to yield a range of scaffolds 519-524 (Scheme 121) [449]. The microflow system was able to rapidly investigate several derivatives allowing the isolatation of 12 examples in low to moderate yields (19-76\%).

In 2018, Gordon and co-workers optimised a continuous-flow protocol for the development of new drug compounds. The authors exploited an H-Cube ${ }^{\circledR}$ Pro system, which can provide high pressures and temperatures, to facilitate an intramolecular DA reaction [450]. The apparatus allowed access to the key intermediate $\mathbf{5 2 6}$ in higher throughput than the previously optimised batch mode $\left(0.002 \mathrm{~g} \mathrm{~h}^{-1}\right.$ vs $\left.0.035 \mathrm{~g} \mathrm{~h}^{-1}\right)$. The obtained scaffold 526 then underwent reduction using the same $\mathrm{H}-\mathrm{Cube}{ }^{\circledR}$ Pro but using different cartridges (Scheme 122). This example shows how a system can be easily reconfigured for different flow purposes.

Inverse-electron demand hetero-DA reactions require high temperatures to occur, therefore high boiling point solvents are often needed, many of these are inherently toxic and obviously difficult to remove. The works of Britton and Martin show how flow chemistry can offer simple ways to address this issue

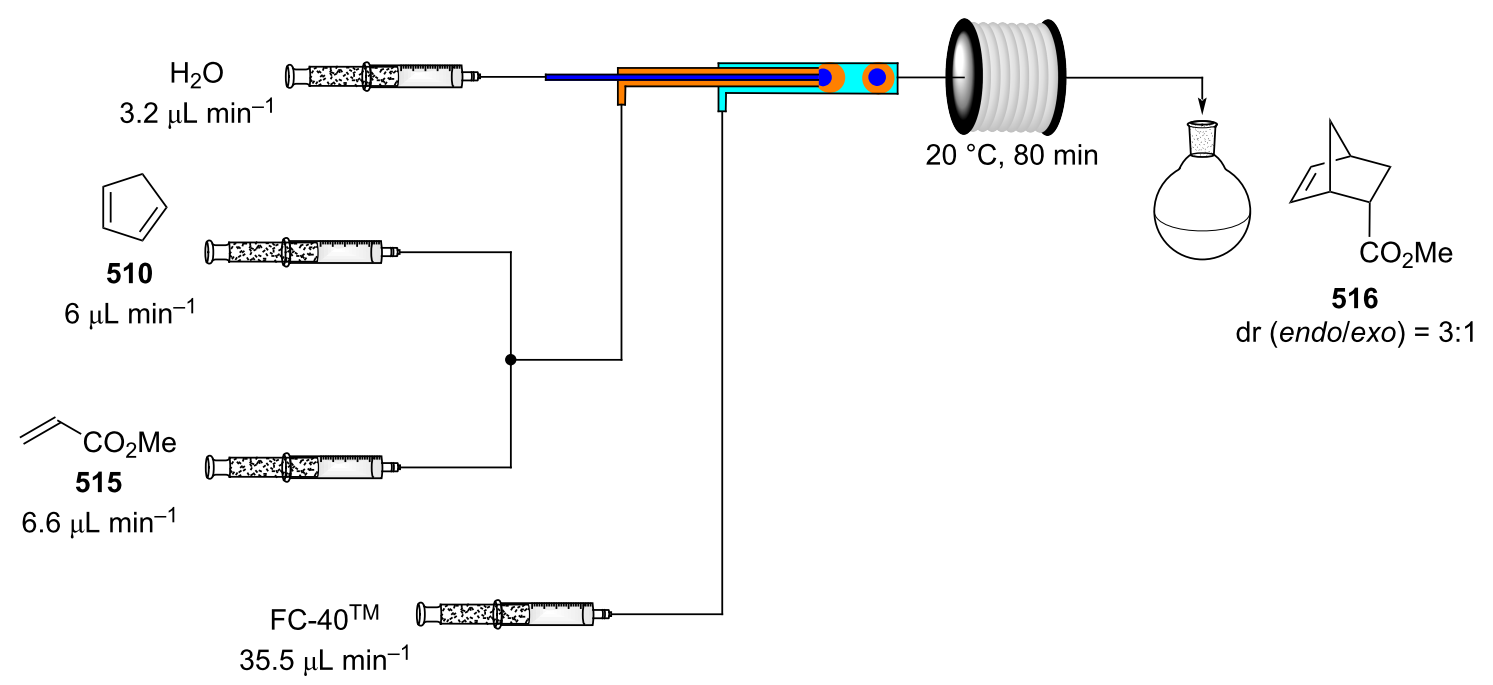

Scheme 120: Continuous-flow DA reaction between 510 and 515 using a shell-core droplet system. 


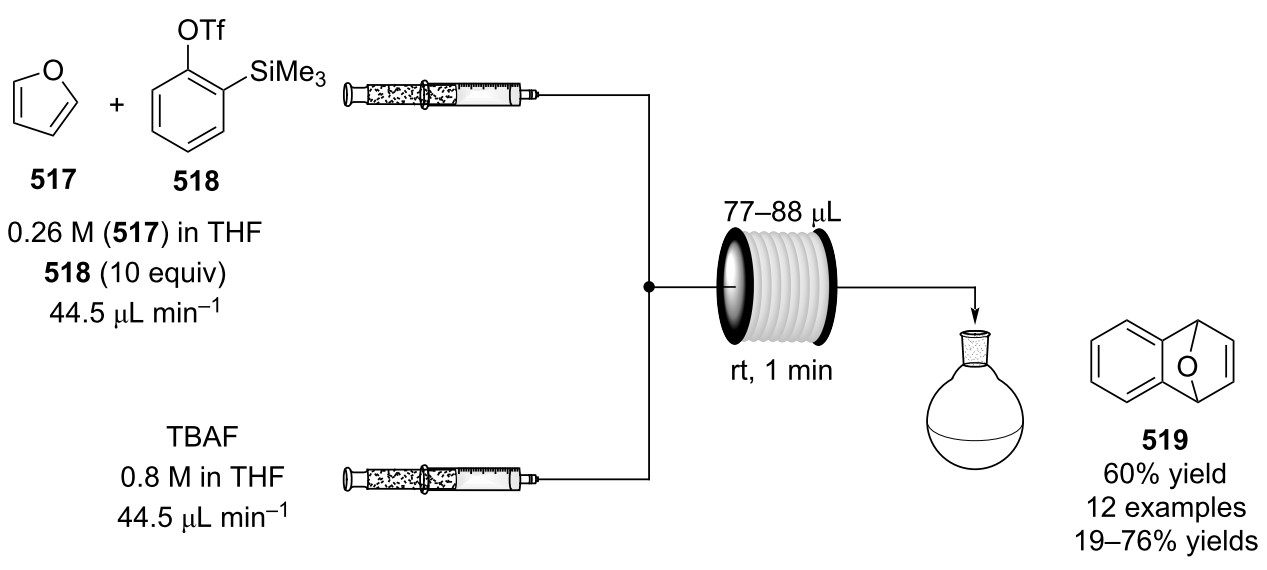<smiles>Cc1ccc2c(c1)C1C=CC2O1</smiles>

520 $68 \%$ yield<smiles>Cc1ccc2c(c1)C1C=CC2N1C(=O)O</smiles>

521 $59 \%$ yield<smiles>C1=CC2OC1c1ccc3ccccc3c12</smiles>

522 $71 \%$ yield<smiles>CN(c1ccccc1)c1cccc2ccccc12</smiles>

523

$19 \%$ yield<smiles>PN(P)c1cccc2ccccc12</smiles>

524

$21 \%$ yield

Scheme 121: Continuous-flow synthesis of bicyclic systems from benzyne precursors.

[451,452]. In these examples, they employed a lower boiling point solvent such as toluene at high temperature $\left(250^{\circ} \mathrm{C}\right)$ and high pressure (750 psi) [452]. This was achieved using a modified GC oven with a stainless steel coil reactor inserted inside. The mixture of $\mathbf{5 2 9}$ and $\mathbf{5 3 0}$ in toluene was pumped through the coil reactor heated at $230{ }^{\circ} \mathrm{C}$ under a pressure $750 \mathrm{psi}$ (Scheme 123). After 2 hours of residence time, the mixture was passed through a small column filled with silica gel and glass wool to prevent any clogging of the BPR. The flow system allowed to isolated the pyridine derivative $\mathbf{5 3 1}$ in 55\% yield. The authors adopted the apparatus for the preparation of other pyridine derivatives yielding low to good results (11-63\% yield).

A microwave flow reactor has been employed in the cycloaddition of furan (486) and diethyl acetylenedicarboxylate enabling the synthesis of the bicyclic structure in good yields and short residence times of around 1 minute [453]. Although promising this area needs additional research in order to streamline the sequence with other potentially interesting modern strategies for DA catalysis such as carbocation catalysis [454].
Retro-DA reactions have been extensively employed for the synthesis of heterocyclic scaffolds. The reaction requires high temperatures and the setup to avoid degradation process over the desired retro-addition. Flow processing enables precise optimisation of the thermal conditions and because of the reactor containment and applied system pressure the use of low-boiling point solvents become viable. In an example preparation the pyrimidinone scaffolds 532-536 was obtained from a retro-DA of the corresponding tricyclic structures (Scheme 124) $[455,456]$. Using the appropriate solvents in flow under high pressure (BPR 300 bar) the desired pyrimidinone products could be isolated in higher yields compared to the corresponding batch reaction.

Several $[2+3]$ cycloadditions have been developed in continuous-flow over the years, and some of these have been recently reviewed, such as the copper-catalysed version of Huisgen reaction [457-459]. The latter consists of a 1,3-dipolar cycloaddition between an azide and alkyne to form the 1,2,3-triazoles as a mixture of 1,4- and 1,5-adducts. To ensure the formation of only one regioisomer, few metal-catalysed versions have been 


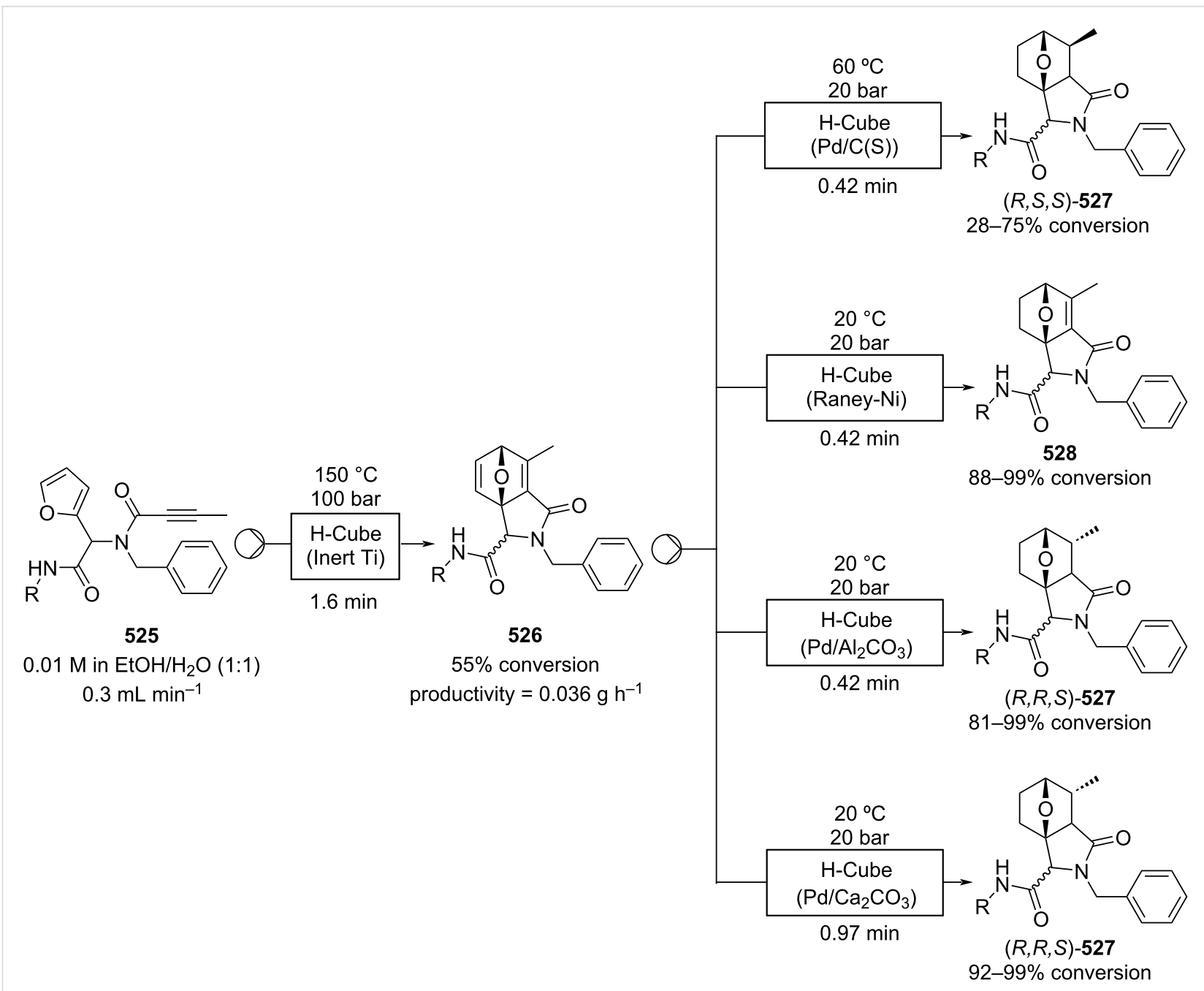

Scheme 122: Continuous-flow synthesis of bicyclic scaffolds 527 and $\mathbf{5 2 8}$ for further development of potential pharmaceutical compounds.

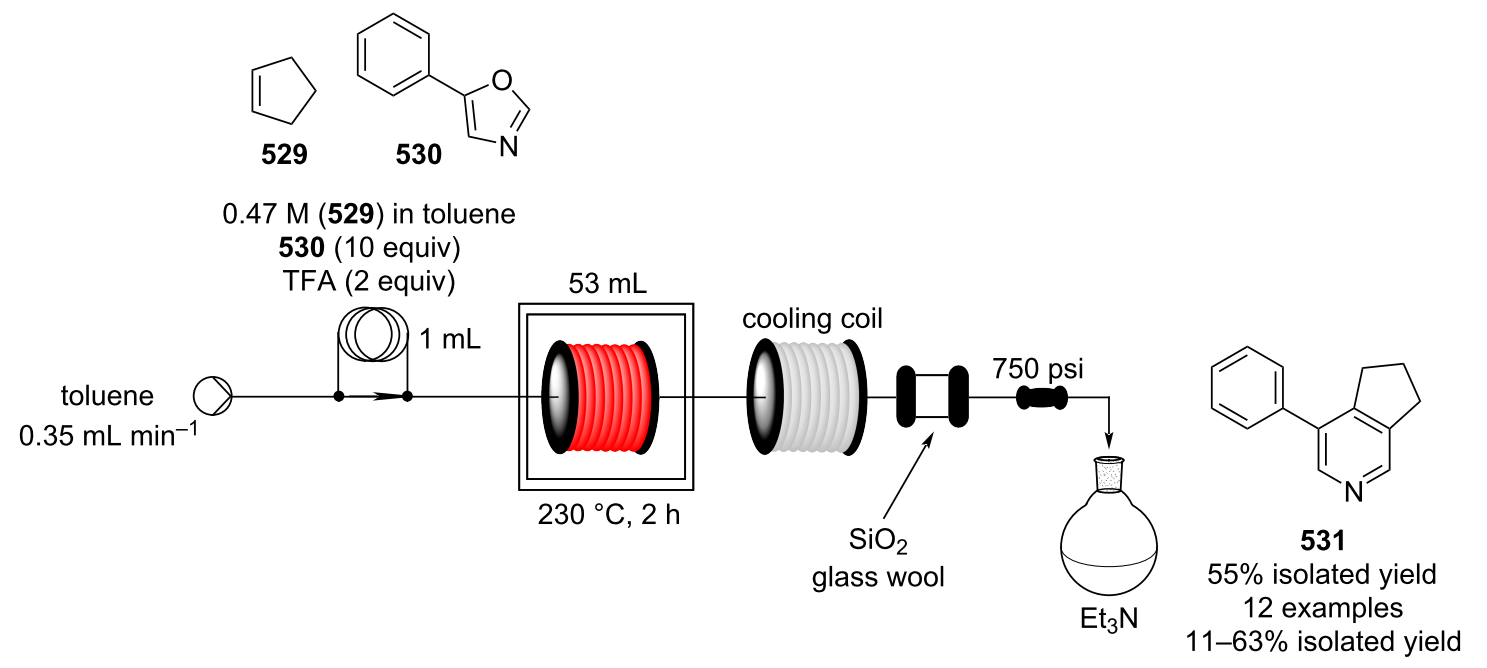

Scheme 123: Continuous-flow inverse-electron hetero-DA reaction to pyridine derivatives such as $\mathbf{5 3 1}$. 

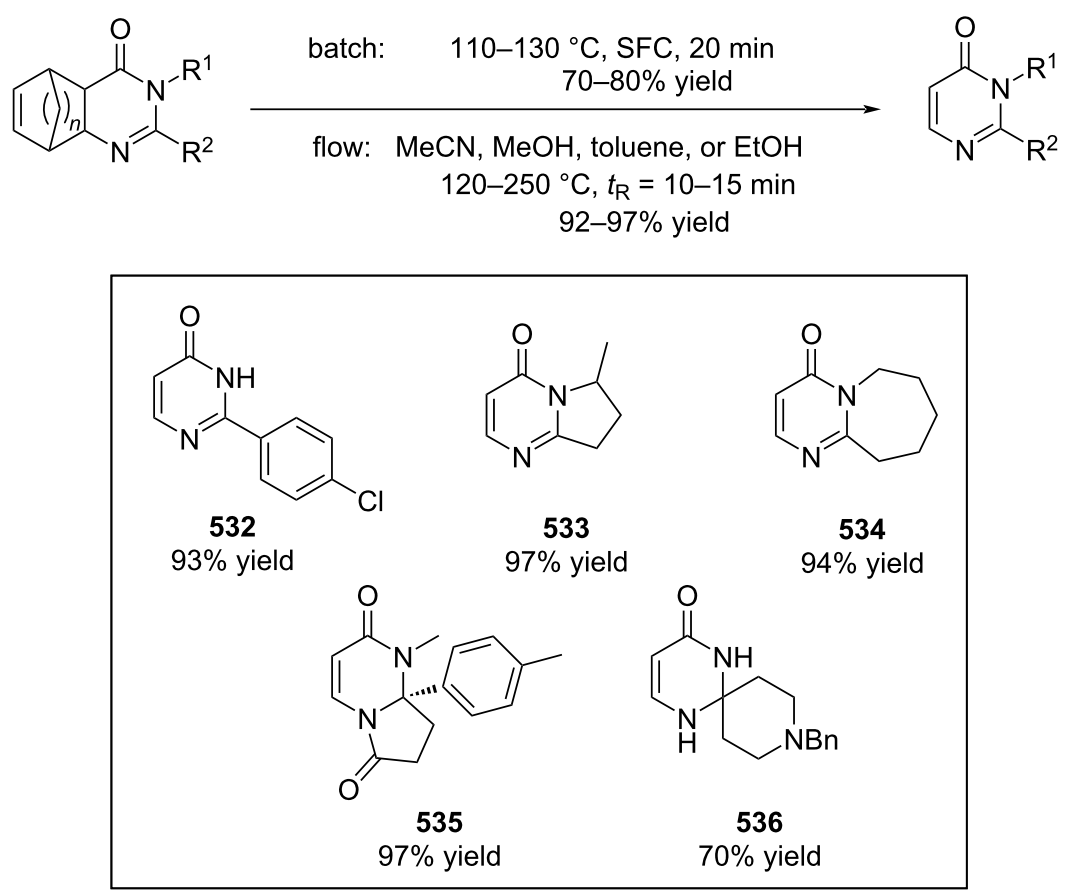

Scheme 124: Comparison between batch and flow for the synthesis of pyrimidinones 532-536 via retro-DA reaction using overheating conditions.

developed [460-462]. The CuAAC (Cu-catalysed alkyne-azide cycloaddition) allows the selective formation of the 1,4-adduct and has found a lot of interest particularly in medicinal chemistry. Several flow intensified processes have been described in the last few years permitting a reduction in reaction times due to the improved mixing and precise control of the reaction condi- tions. Raić-Malić and co-workers assembled a continuous-flow reactor coupled with an ultrasound system for the synthesis of L-ascorbic acid derivatives such as $\mathbf{5 0 5}$ (Scheme 125) [463].

The output stream was collected into a batch reactor and quenched using a benzylamine scavenger resin. With this appa-

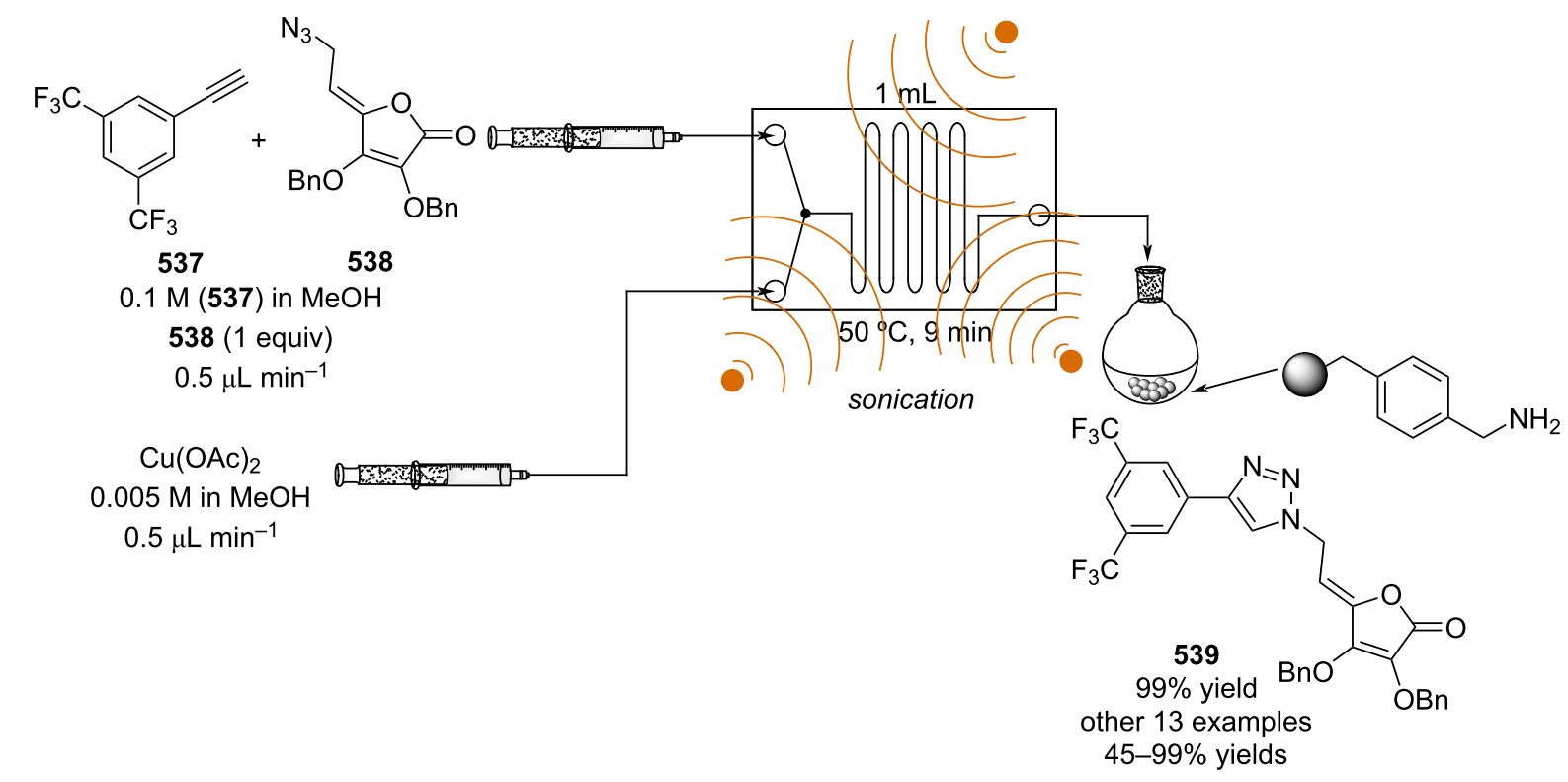

Scheme 125: Continuous-flow coupled with ultrasonic system for preparation of L-ascorbic acid derivatives 539 developed by Raić-Malić et al. 
ratus, the reaction times were reduced from hours to minutes, increasing the throughput and reducing the time for the preparation of a compound series.

Flow chemistry also enables better handling of the hazardous azide reagents [464,465]. In 2016, Vögtle et al. optimised a continuous preparation of 2-chloroazides to utilise in CuAAC (Scheme 126) [466].

The system employed one of the most challenging reagents to use, chlorine azide $\left(\mathrm{ClN}_{3}\right)$. The reagent was prepared in situ and directly utilised for the 1,2-azidochlorination of the olefin $\mathbf{5 4 0}$ After the reaction, the excess azide was quenched in-line using $\mathrm{NaHCO}_{3}$ and the resulting biphasic mixture separated in flow using a Zaiput membrane separator. The product $\mathbf{5 4 2}$ was obtained as a $0.2 \mathrm{M}$ solution which was diluted for the $\mathrm{CuAAC}$ reaction. To enact the coupling a copper coil reactor was employed, using a $0.1 \mathrm{M}$ solution which increased the substrate-tocatalyst ratio, improving the conversion. The flow system enabled isolation of $\mathbf{5 4 3}$ via solvent evaporation in $83 \%$ yield in a throughput of $542 \mathrm{mg} \mathrm{h}^{-1}$.

Immobilisation of copper catalysts has been further developed; alongside the usage of copper coils, already described by Tranmer and Fülöp [457,458,467,468], other new heterogeneous formats have also been reported [469-473]. As an example, in 2020, a polymer-supported tris-triazole ligand was prepared and utilised for $\mathrm{Cu}$ immobilisation [474]. The CuAAC was carried out in a monolith and a packed-bed reactor for comparison, and the latter proved to be more efficient, probably due to a higher contact area. To form the active $\mathrm{Cu}(\mathrm{I})$ species, sodium L-ascorbate was continuously streamed along with the reac- tants (Scheme 127). The packed reactor was investigated for the preparation of 8 substrates and the catalyst showed reduced activity only after roughly 20 hours of operation providing STYs of $63-250 \mathrm{~g} \mathrm{~L}^{-1} \mathrm{~h}^{-1}$.

Silver-catalysed alkyne-azide cycloaddition (AgAAC) have also been investigated under flow conditions. In 2017, Zhang and co-workers developed a immobilised silver catalyst, linked to an $\mathrm{N}$-heterocyclic carbene (NHC) ligand itself tethered to a polyacrylonitrile fibre $(\mathrm{Ag}-557)$ which could be packed into a column reactor for use in flow (Scheme 128) [475]. The authors described a three-component reaction (A3-coupling reaction) catalysed by Ag-557 under flow conditions, along with a domino A3-coupling/AgAAC. In A3-coupling reaction, the column was heated to $60{ }^{\circ} \mathrm{C}$ and after a 16 minutes residence time compound $\mathbf{5 5 8}$ was isolated in $95 \%$. The flow apparatus was run for 24 hours giving no signs of catalyst deactivation with a productivity of $\mathbf{5 5 8}$ of $28 \mathrm{mmol} \mathrm{h}^{-1}$. The domino A3-coupling/AgAAC reaction was setup at a higher temperature $\left(120^{\circ} \mathrm{C}\right)$ to complete the reaction. The use of a back pressure regulator (BPR) avoid the need to change the solvent as the pressure is increased to $500 \mathrm{psi}$.

Along with the Huisgen-type reactions, many other 1,3-dipolar cycloadditions have been developed in flow over the years. Flow conditions have found great utility in the synthesis of a diverse range of scaffolds such as pyrrolidines [476-479], pyrroles [480], pyrazoles [481-483], tetrazoles [484,485], isoxazoles [486-488], and bicyclic compounds [489-491].

In addition [2 +2$]$ cycloadditions have also been of great interest in organic synthesis as cyclobutanes are key intermedi-

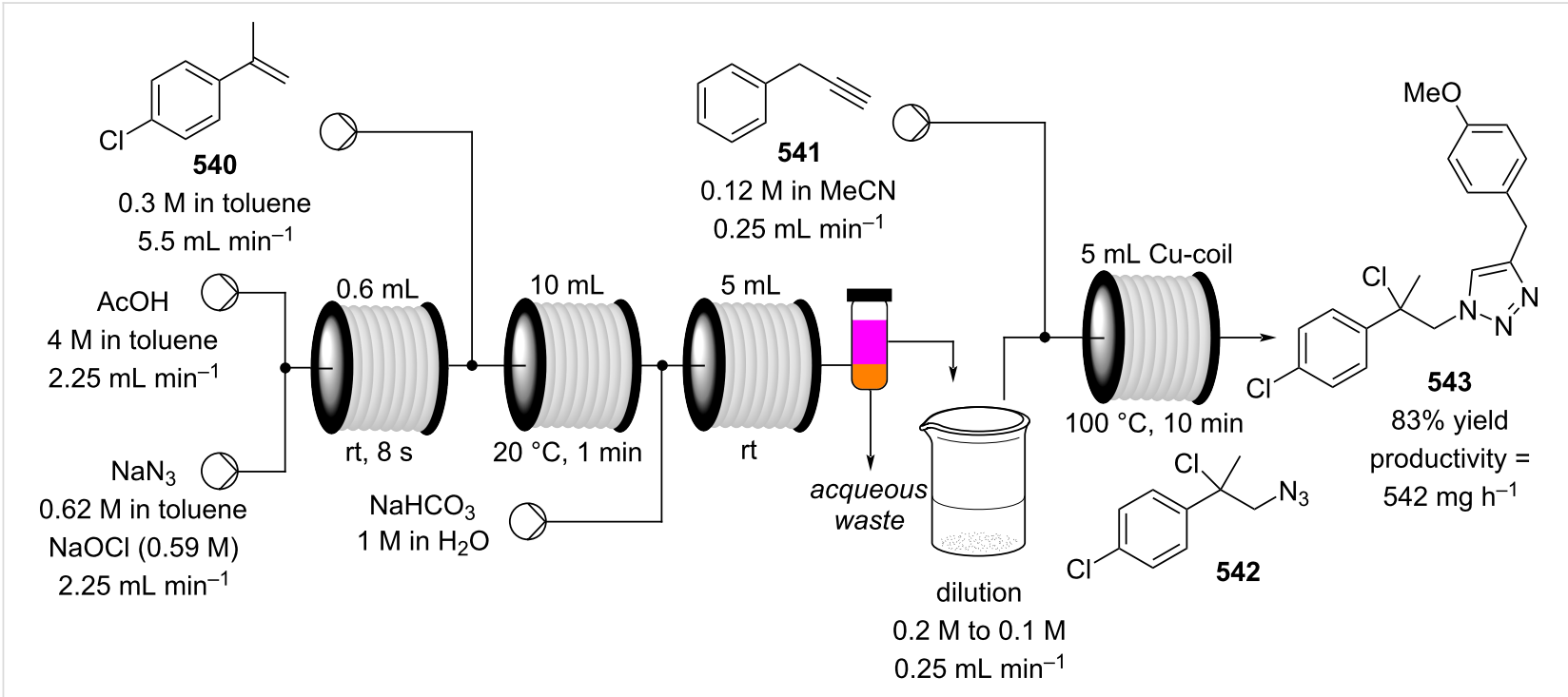

Scheme 126: Two-step continuous-flow synthesis of triazole $\mathbf{5 4 3 .}$ 


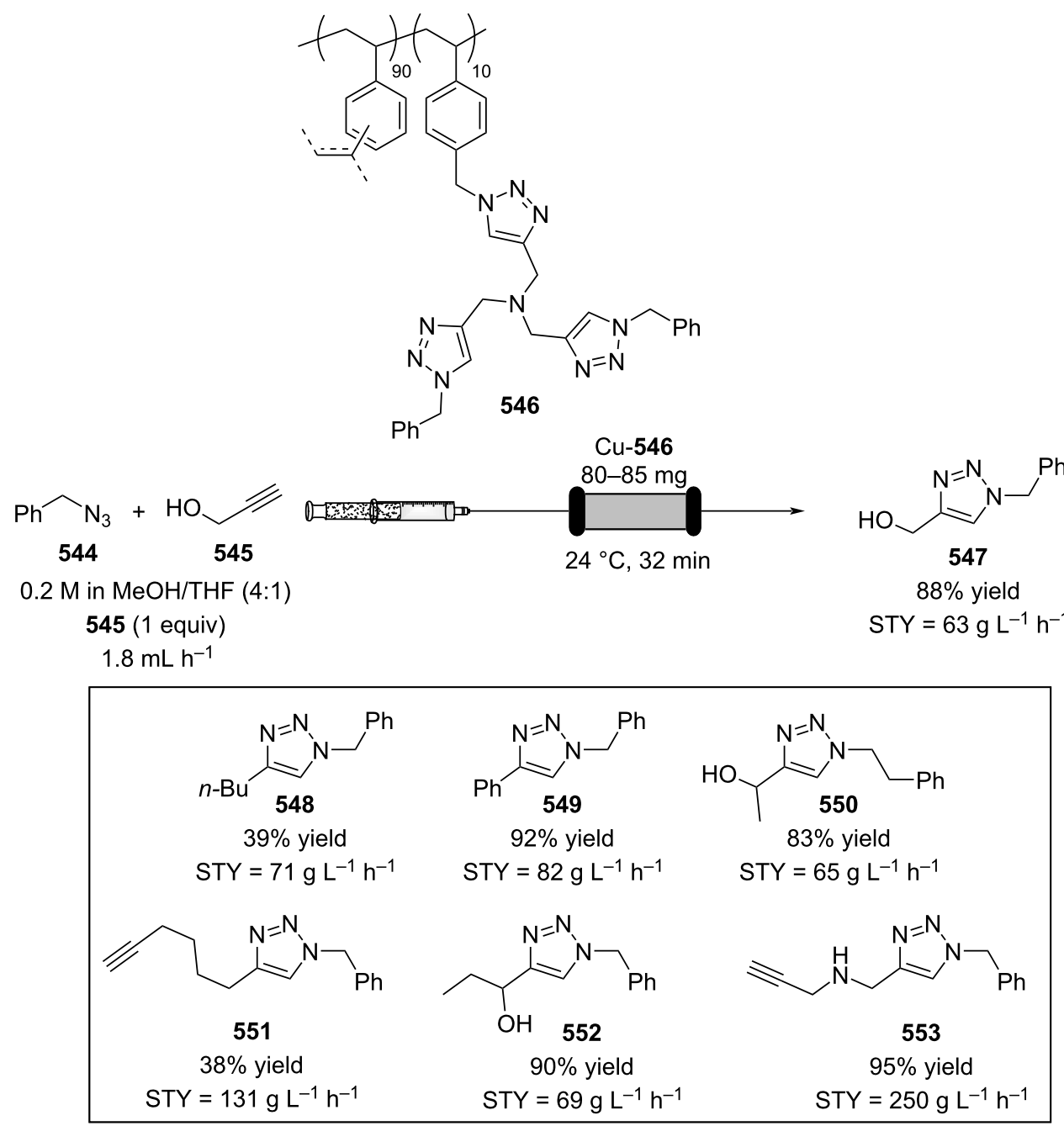

Scheme 127: Continuous-flow preparation of triazoles via CuAAC employing 546-based heterogeneous catalyst.

ates for the preparation of larger rings (via ring expansion) as well as enabling formation of interesting 4-membered rings such as lactam and lactones [492,493]. The [2+2] cycloadditions involve an olefin at its singlet/triplet excited state (SOMO) and a alkene at its ground state (LUMO) [494]. Photoreactions performed in batch mode present various limitations, such as low selectivity, and long reaction times. Since the light absorption for a photoreaction performed in a flask follows the Lambert-Beer law, these issues can be attributed to poor irradiation intensity. To overcome these problems, thin, low volume glassware has been developed, however, only small batches can be run resulting in low productivity. For these reasons, microfluidic flow reactor technology has been shown to be a good fit to reduce the reaction times and increase the efficiency, therefore gaining the desired throughputs [495-497]. Many examples of customised flow photoreactors have been developed and investigated for [2+2] cycloadditions [316,498-506]. In one of the early examples, Fukuyama, Ryu, and co-workers developed a continuous-flow photochemical $[2+2]$ cycloaddition between enones and vinyl acetate (Scheme 129) [502]. The system utilised a glass microreactor irradiated through one side of the device where the reactants undergo irradiation for 2 hours prior to collection. After confirming the efficiency of the approach compared to the batch one ( $88 \%$ vs $8 \%$ yield), the authors investigated other derivatives, also yielding the desired materials in moderate to good yields $(47-70 \%)$.

Working in flow also provides improved options for scaling of photochemical transformations. In 2005, Booker-Milburn et al. developed a flow reactor that could perform a large scale production run of a [2+2] cycloadduct 524 (Scheme 130) [316].

In this example, a customised immersion well made in Vycor was tightly wrapped with a fluorinated ethylenepropylene (FEP) 
<smiles>C#Cc1ccccc1</smiles>

554

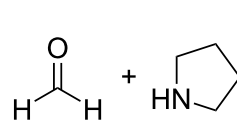

555

$1 \mathrm{M}(556)$ in $\mathrm{MeCN}$

554 (1.2 equiv)

555 (1.2 equiv)

$0.5 \mathrm{~mL} \mathrm{~min}^{-1}$

556

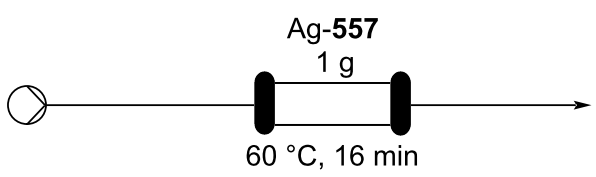<smiles>N#CC(=O)NCCCNC(=O)CN1C=CN(Cc2ccccc2)C1[Ge]Cl</smiles><smiles>C(#Cc1ccccc1)CN1CCCC1</smiles>

558

$95 \%$ yield over $24 \mathrm{~h}$ productivity $=28 \mathrm{mmol} \mathrm{h}^{-1}$ 6 examples 80-95\% yield<smiles>C#Cc1ccccc1</smiles>

554

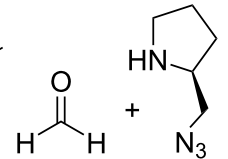

555

559

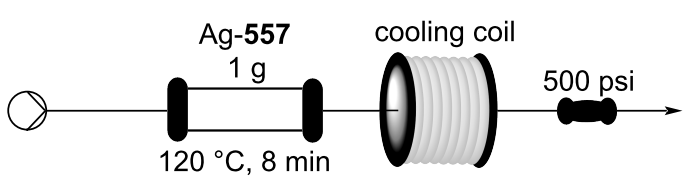

$1 \mathrm{M}(559)$ in $\mathrm{MeCN}$

554 (1.2 equiv)

555 ( 1.2 equiv)

$0.2 \mathrm{~mL} \mathrm{~min}^{-1}$

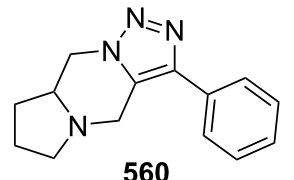

$87 \%$ yield

2 examples $80-87 \%$ yield

Scheme 128: Continuous-flow synthesis of compounds 558 through A3-coupling and $\mathbf{5 6 0}$ via AgAAC both employing the polyacrylonitrile-supported catalyst Ag-557.

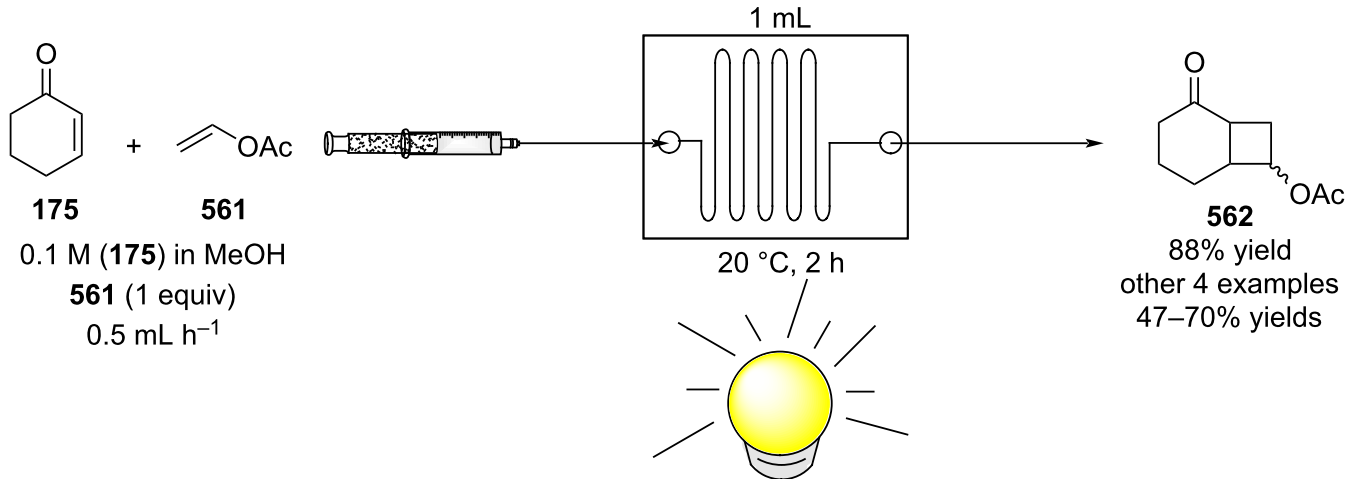

$\mathrm{Hg}, 300 \mathrm{~W}$

Scheme 129: Continuous-flow photoinduced [2+2] cycloaddition for the preparation of bicyclic derivatives of 562.

tubing ( $2.7 \mathrm{~mm}$ i.d.). Three layers of tubing were coiled around the well, in which was placed a $400 \mathrm{~W}$ medium-pressure mercury vapour discharge lamp. The input stream flowed from the outermost layer to the innermost one at a flow rate of $4 \mathrm{~mL} \min ^{-1}$. Using these conditions, a $0.1 \mathrm{M}$ solution of maleimide (563) and 1-hexyne (564) were combined and reacted over 24 hours through the flow reactor, gaining $85 \mathrm{~g}$ of the desired material 565 (isolated yield $82 \%$ ). The authors also confirmed the usage of a higher power lamp $(600 \mathrm{~W})$ allowed an increase in the reaction concentration to $0.4 \mathrm{M}$ with double the flow rate at $8 \mathrm{~mL} \mathrm{~min}{ }^{-1}$ to gain a projected throughput of $685 \mathrm{~g}$ $\mathrm{d}^{-1}$ when running uninterrupted. The same flow system was also employed for a [5+2] cycloaddition for the preparation of azepines 568 and $\mathbf{5 6 9}$, obtaining again higher throughputs compared to the batch mode.

The same group has also investigated a series of other [2 +2] cycloadditions demonstrating their scale-up using flow condi- 

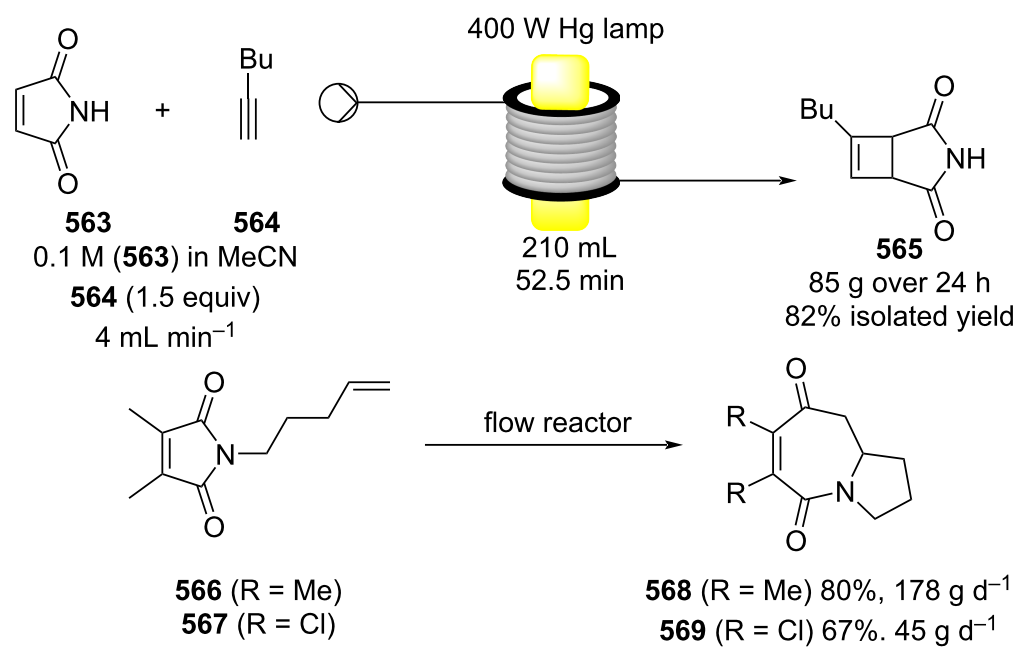

Scheme 130: Continuous-flow [2 +2$]$ and [5 +2$]$ cycloaddition on large scale employing a flow reactor developed by Booker-Milburn and co-workers.

tions [507-509]. As an example, in 2013, they developed a methodology for the synthesis of the aza-tricyclic structure 571, starting from a $[2+2]$ cycloaddition reaction of pyrrole $\mathbf{5 7 0}$ (Scheme 131) [510].

In order to increase the throughput, a flow apparatus was employed allowing roughly $8 \mathrm{~g}$ of final material to be isolated after 24 hours of runtime (57\% isolated yield). The same flow setup used for various other analogues enabled the intermediate structures to be exploit in the synthesis of more complex natural products, such as (+)-3-demethoxyerythratidinone (573) and the lycorane alkaloids (illustrated by compound 574) [511,512].
In 2015, Beeler et al. customised a photoreactor where a xenon light source was filtered and focused into a 2 inch beam. A conical coil reactor was placed in front of the light and kept refrigerated. Utilising this flow system, the group developed a thiourea-catalysed [2 +2$]$ photocycloaddition of cinnamates (Scheme 132) [503].

The first setup consisted of an acetonitrile solution of the reactants passing through the conical flow reactor. However, more recent findings showed that biphasic conditions were more efficient, with slug flow leading to a reduced residence time and higher throughput [504]. Of particular value was that the flow

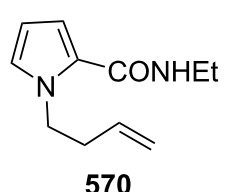

$5 \mathrm{mM}$ in $\mathrm{MeCN}$

$9.5 \mathrm{~mL} \mathrm{~min}^{-1}$
36 W PL-L lamp

$(254 \mathrm{~nm})$

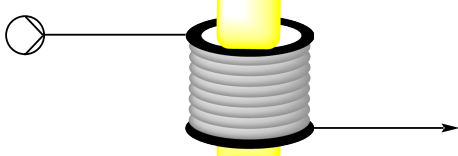

$9.5 \mathrm{~mL}$

$1 \mathrm{~h}$

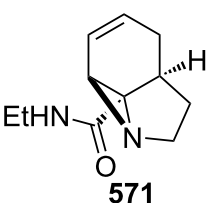

$57 \%$ isolated yield throughput $7.9 \mathrm{~g} \mathrm{~d}^{-1}$<smiles>COc1cc2c(cc1OC)[C@]13CCC(=O)C=C1CCN3CC2</smiles><smiles>CCCC1CCCCC1C</smiles><smiles>CC(C)(C)OC1C2C=CCC1(C)C1CNCC21</smiles><smiles>[C+]1C=CC=C1</smiles>

$39 \%$ isolated yield<smiles>[R5]CN1CCC2CCCC3c4cc5c(cc4C2C31)OCO5</smiles>
throughput $7.37 \mathrm{~g} \mathrm{~d}^{-1}$ 

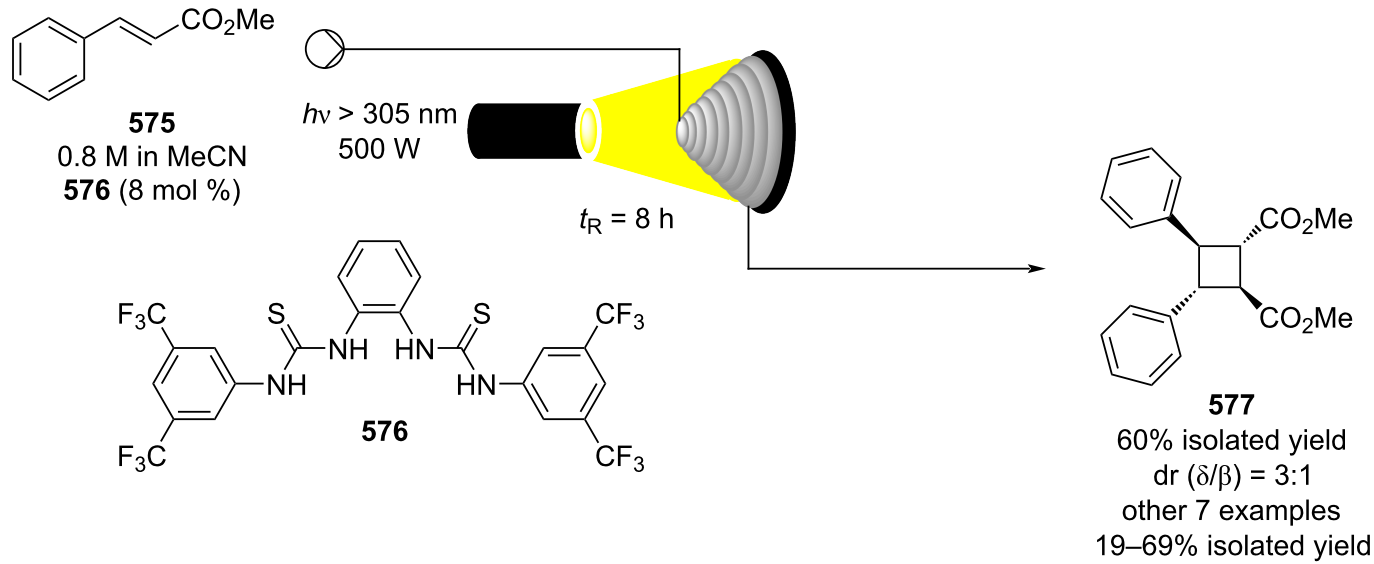

Scheme 132: Continuous-flow [2 +2$]$ photocyclization of cinnamates.

system enabled operation with particularly sensible cinnamates, which had proved problematic during monophasic reactions in batch. The authors attributed these outcomes to the improved mixing, and the smaller UV path length enhancing catalyst-substrate interactions and controlling UV absorbance. These conditions have also proven beneficial when employed for a Paternò-Büchi photoreaction as described by Nishiyama, Kakiuchi and co-workers [513].

Many of the photoinduced reactions employ pressurized mercury lamps as UV light sources. However, they present numerous drawbacks such as high energy consumption, low efficiency (including long start up times), a wide wavelength spectrum, and short bulb lifetimes [514]. As the use of sunlight would be highly desirable, the inhomogeneity of the solar irradiation over time and space makes it quite unfeasible for indus- trial applications. Therefore, great interest has been seen in the area of high efficiency narrow wavelength light-emitting diodes (LED) as alternative light sources. These systems provide several advantages over the pressurized mercury lamps, although their light spectrum are mainly limited to long wavelengths $(>340 \mathrm{~nm})$. To shorten this wavelengths, a catalytic amount of a triplet photosensitizer can be employed, which shortens the long wavelengths to higher-energy waves which is synthetically useful for reactions such as $[2+2]$ cycloadditions and other photoreactions.

As an example, Kappe et al. developed a [2+2] cycloaddition of maleic anhydride (578) and ethylene using $375 \mathrm{~nm}$ LED's as the light source and thioxanthone (579) as a photosensitizer (Scheme 133) [515]. The authors first optimised a flow version using benzophenone, however, higher catalyst loading

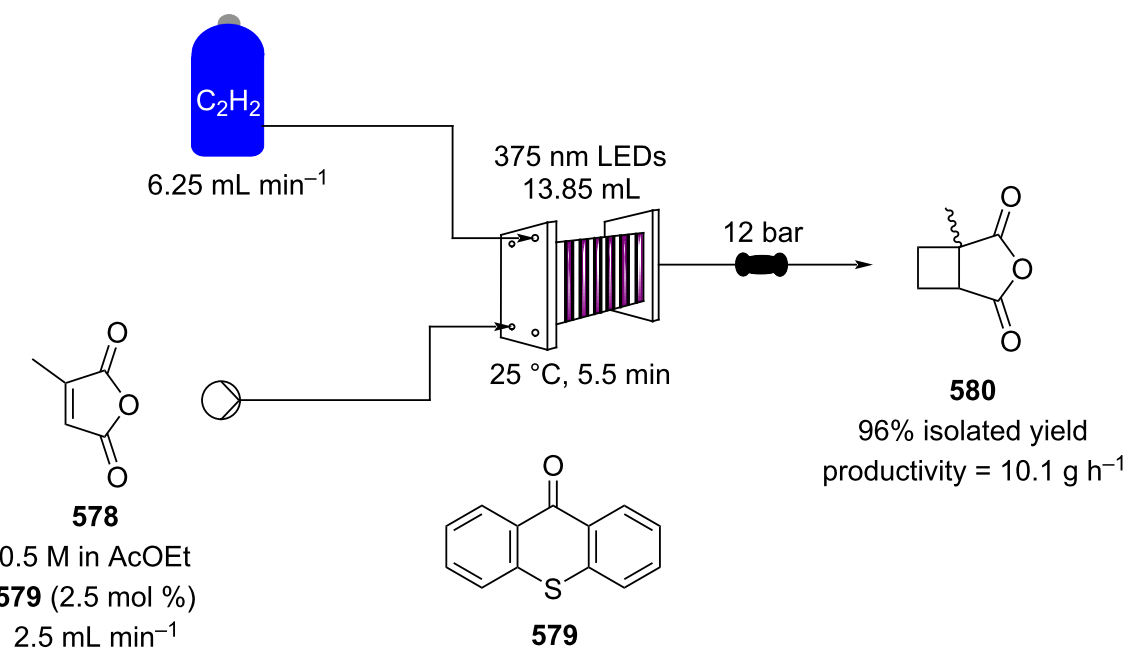

Scheme 133: Continuous-flow preparation of cyclobutane 580 on a 5-plates photoreactor. 
(40 mol \%) required an additional purification step at the end. Following optimisation, a $0.5 \mathrm{M}$ solution of methyl maleic anhydride (578) and $2.5 \mathrm{~mol} \%$ thioxanthone (579) in ethyl acetate was mixed with ethylene gas and directed into a $2.77 \mathrm{~mL}$ platebased reactor. An array of $375 \mathrm{~nm}$ LEDs was used to irradiate the photoreactor to furnish the cyclobutane product $\mathbf{5 8 0}$ in more than $99 \%$ conversion and a STY of $759 \mathrm{~g} \mathrm{~L}^{-1} \mathrm{~h}^{-1}$. Stacking five plates in series allowed a 5-fold scaling up of the process to obtain roughly $100 \mathrm{~g}$ of the cyclobutane $\mathbf{5 8 0}$ in $96 \%$ isolated yield across a 10 hours runtime (productivity $10.1 \mathrm{~g} \mathrm{~h}^{-1}$ ) (Scheme 133).

Recently, Zhang, Wang and co-workers employed a polymeric carbon nitride (PCN) material as a heterogeneous photocatalyst for $[2+2]$ cycloadditions under white LED lighting (Scheme 134) [516].

The PCN catalyst was prepared as a deposit on commercial glass beads which were packed into a fixed-bed photoreactor where a solution of $\alpha$-asarone (581) or other styrene derivatives in a solution of nitromethane was flowed through. Even though, the flow system provided a better tool than a reactor batch $(53 \%$ vs $87 \%$ ), interestingly the reaction times were longer for most of the examples explored (24-48 h). This was not investigated or explained but is probably due to reduced catalyst contact times in the flow system.

In 2016, the group of Booker-Milburn developed an innovative photoreactor for the preparation of compounds at the kilogram scale [517]. The apparatus nicknamed "The Firefly" (Figure 11) was made from quartz reaction tubes surrounding a powerful Hg lamp (1.5 kW-5 kW of power) and cooled using fans and a water cooling jacket. The reactor had a $120 \mathrm{~mL}$ internal volume. To increase the light absorption even at the outermost part of the pipes, a reflective metal film was wrapped around the outside of the reactor.

Seven different photochemical transformations were investigated using "The Firefly" device and in all cases high throughputs were achieved (Table 13). Noteworthy to mention is the intramolecular $[2+2]$ cycloaddition of the quinone $\mathbf{5 8 9}$ to Cookson's dione (590) that occurred in high yields and a throughput of roughly $8 \mathrm{~kg}$ of material per day (Table 13, entry 5 ). The authors pointed out how productivity is directly proportional to the power of the light source as the synthesis of the trichloro compound $\mathbf{5 8 5}$ provides $2.85 \mathrm{~g} \mathrm{~h}^{-1}$ in a $400 \mathrm{~W}$ FEP reactor and $28.8 \mathrm{~g} \mathrm{~h}^{-1}$ using $3 \mathrm{~kW}$-powered PTFR.

Silyl-protected hydroxycyclobutanes (e.g., 596) were prepared in flow from silyl enol ethers and $\alpha, \beta$-unsaturated esters via an acid-catalysed [2 + 2] cycloaddition [518]. The orbital symmetry of the reacting partners allows the reaction to proceed without the need for light, however, a catalyst is required. The in situ-formed silyl triflic imide $\left(\mathrm{Tf}_{2} \mathrm{NH}\right)$ employed as the catalyst proved both efficient and selective for a range of different substrates; however, in few examples oligomerization, polymerisation and product decomposition was observed in minor quantities. Takasu and co-workers setup a flow reactor which reduced the reaction time and temperature, thus eliminating the formation of byproducts. The system consisted of two Y-shape

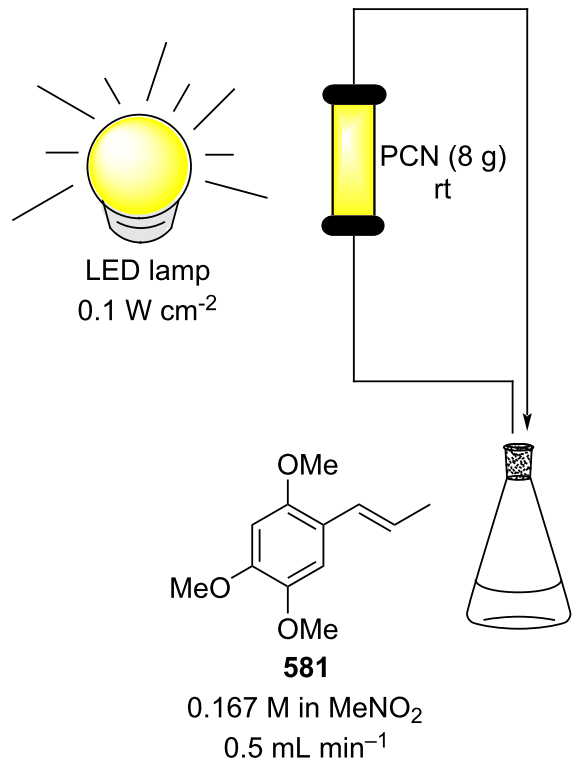

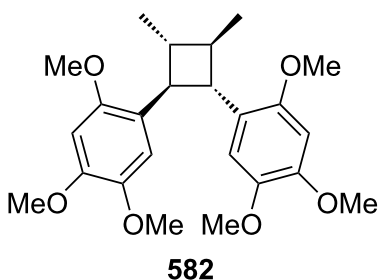

$87 \%$ isolated yield other 13 examples $49-80 \%$ yields 


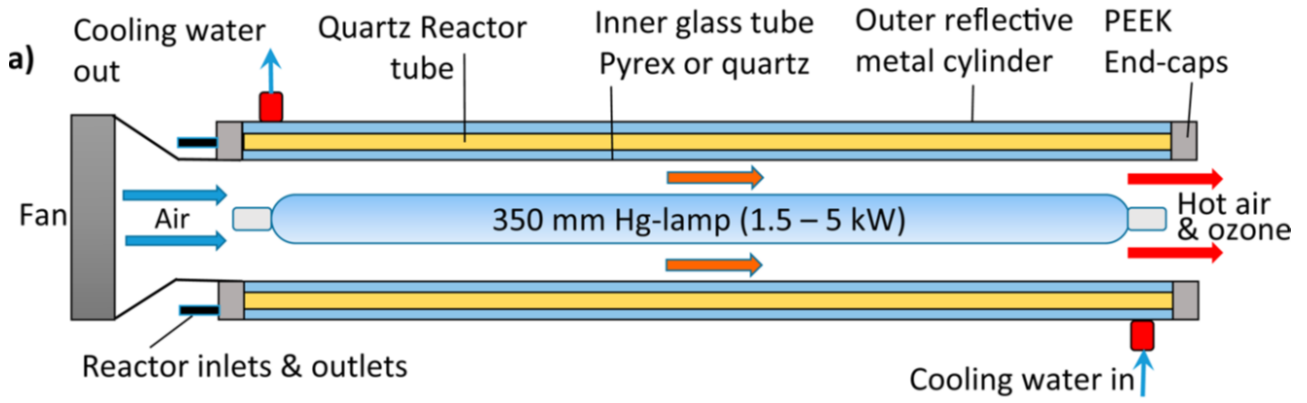

b)

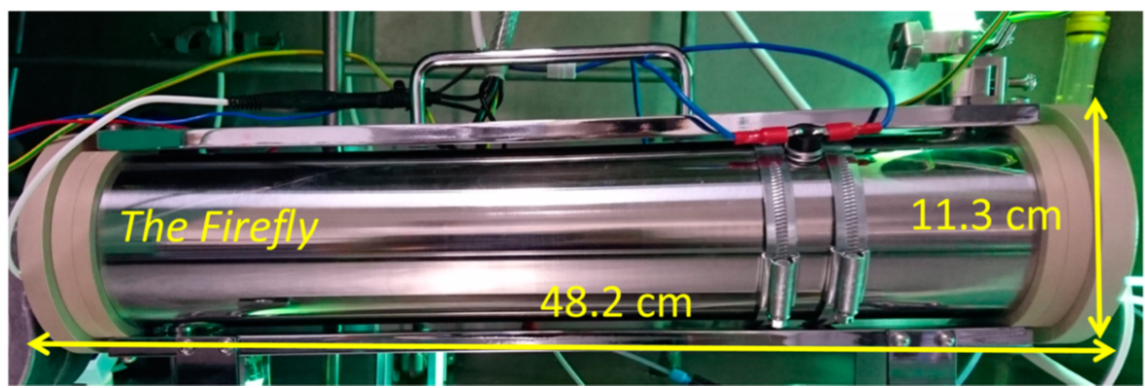

Figure 11: Picture of the parallel tube flow reactor (PTFR) "The Firefly" developed by Booker-Milburn et al. a) Diagram of the photoreactor. b) Rector from the outside [517]. Reprinted with permission from [517]. Copyright 2016 American Chemical Society.

Table 13: Photochemical reactions exploited using the parallel tube flow reactor "The Firefly".

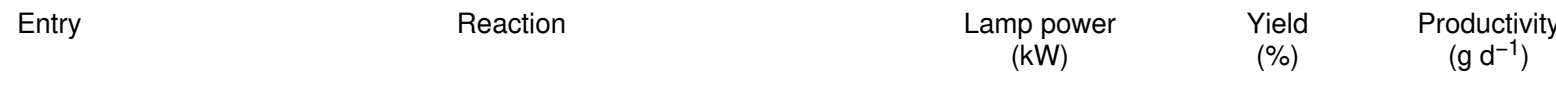

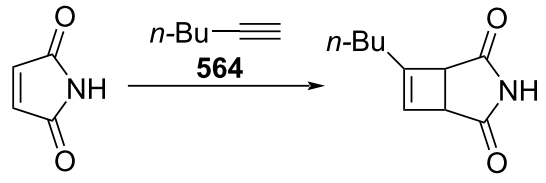

565<smiles>C#CCCCCCO</smiles>

563

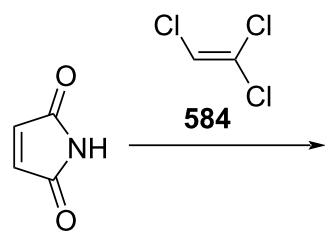

563<smiles>O=C1OC(=O)C2=C1CCCC2</smiles><smiles>O=C1NC(=O)C2C(CO)=CC12</smiles>

583<smiles>O=C1NC(=O)C2C1C(Cl)C2(Cl)Cl</smiles>

586

4

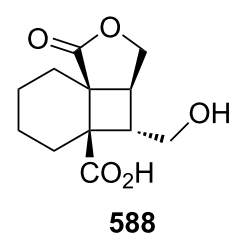

3

66

691 
Table 13: Photochemical reactions exploited using the parallel tube flow reactor "The Firefly". (continued)

5<smiles>O=C1C=CC(=O)C2C3C=CC(C3)C12</smiles><smiles></smiles>

589<smiles>O=C1CC2CC3CC2C(O)C13</smiles>

590

\section{5}

3

3
89

89

8,058<smiles>C=CCN(C(=C)c1ccccc1)C(=O)c1ccccc1</smiles>

592<smiles>O=C1c2ccccc2C2(O)CCCN12</smiles>

594 micromixers and two reaction microtubes. The silyl enol ether was first mixed with the $\mathrm{Tf}_{2} \mathrm{NH}$ to form the active silyl triflic imide catalyst. The reaction mixture then merged with the enophile $\mathbf{5 1 5}$ to yield the desired cyclobutane. Under flow conditions, the reaction occurred at higher temperature (rt vs $-78{ }^{\circ} \mathrm{C}$ ) and faster reaction times (12 s vs $2 \mathrm{~h}$ ) compared with the optimised batch reactor. The ability to efficiently control the reaction timing, thereby limiting the formation of undesired side products, and to adjust the residence times for less reactive substrates enhanced the yields. In this way, more Lewis acid sensitive silyl enol ethers could be used and the desired cyclobutanes such as 596-599 were isolated in good yields (Scheme 135).

Staudinger $[2+2]$ cycloaddition for the synthesis of $\beta$-lactams occurs between an imine and ketene. The latter is a highly reac-

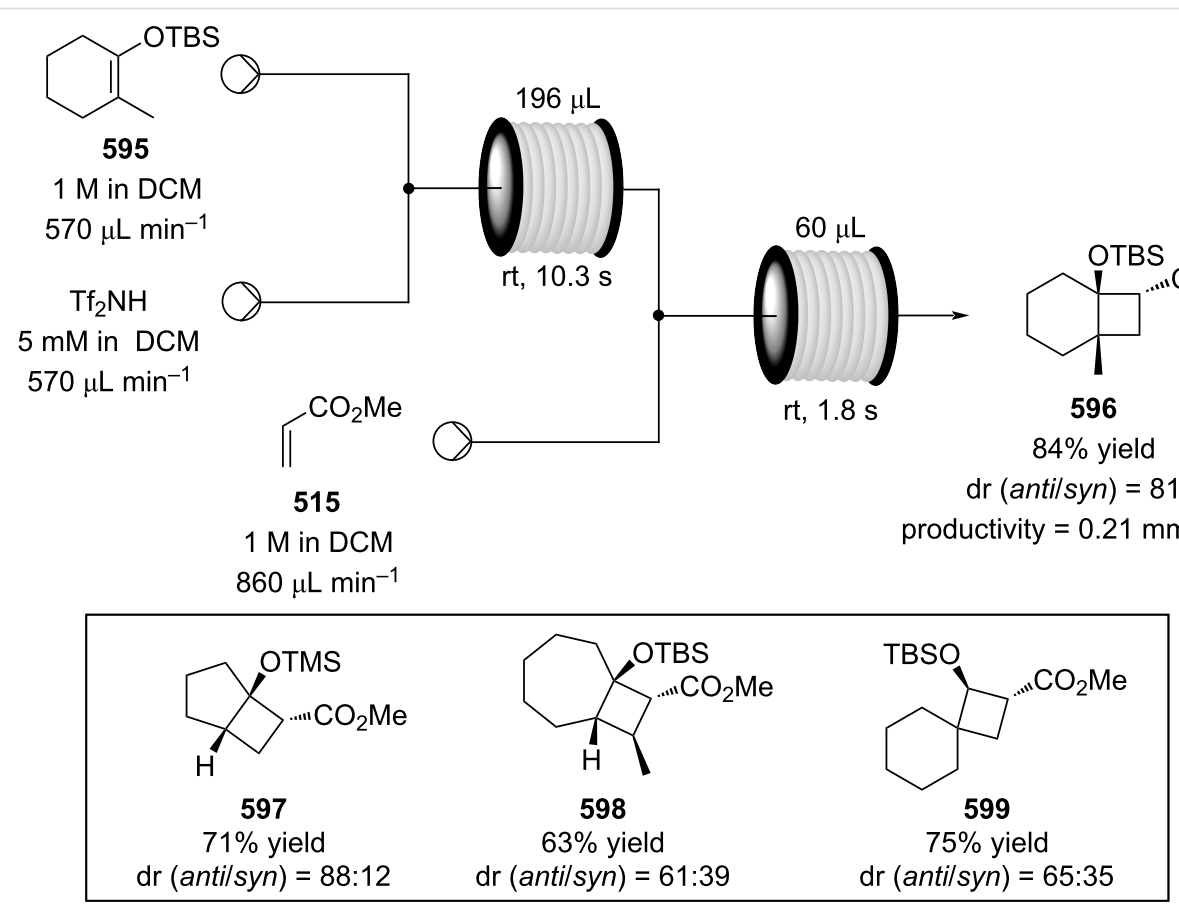

Scheme 135: Continuous-flow acid-catalysed [2 + 2] cycloaddition between silyl enol ethers and acrylic esters. 
tive species usually generated in situ to avoid decomposition through dimerization or polymerisation. As flow chemistry allows to efficiently handle reactive materials, several flow systems have been described over the years to aid this chemistry. A flow approach was first reported by Lectka et al., the system consisted of two jacketed columns joined together and filled with polymer-supported reagents (Scheme 136) $[519,520]$. In the first glass column the ketene was formed from an acyl chloride 600 using the BEMP-resin 603 as base at $-78{ }^{\circ} \mathrm{C}$. The outflow was directed into the second column where it was combined with a stream of $\alpha$-imino ester $\mathbf{6 0 1}$ (solution in THF). The desired [2+2] cycloaddition took place at a reduced temperature of $-43{ }^{\circ} \mathrm{C}$ using a solid-phase chiral catalyst 604. After 2 hours complete elution of the reaction mixture occurred and the lactam product $\mathbf{6 0 2}$ was isolated enantiomerically pure in $65 \%$ yield.

Ley and Hafner described a different approach for the ketene generation. Starting from $\alpha$-haloacyl halides such as $\mathbf{6 0 5}$, the ketene was prepared by a zinc-mediated dehalogenation [521]. The flow system employed a zinc-packed column at $\mathrm{rt}$ (Scheme 137). The generation of the reactive species was con-

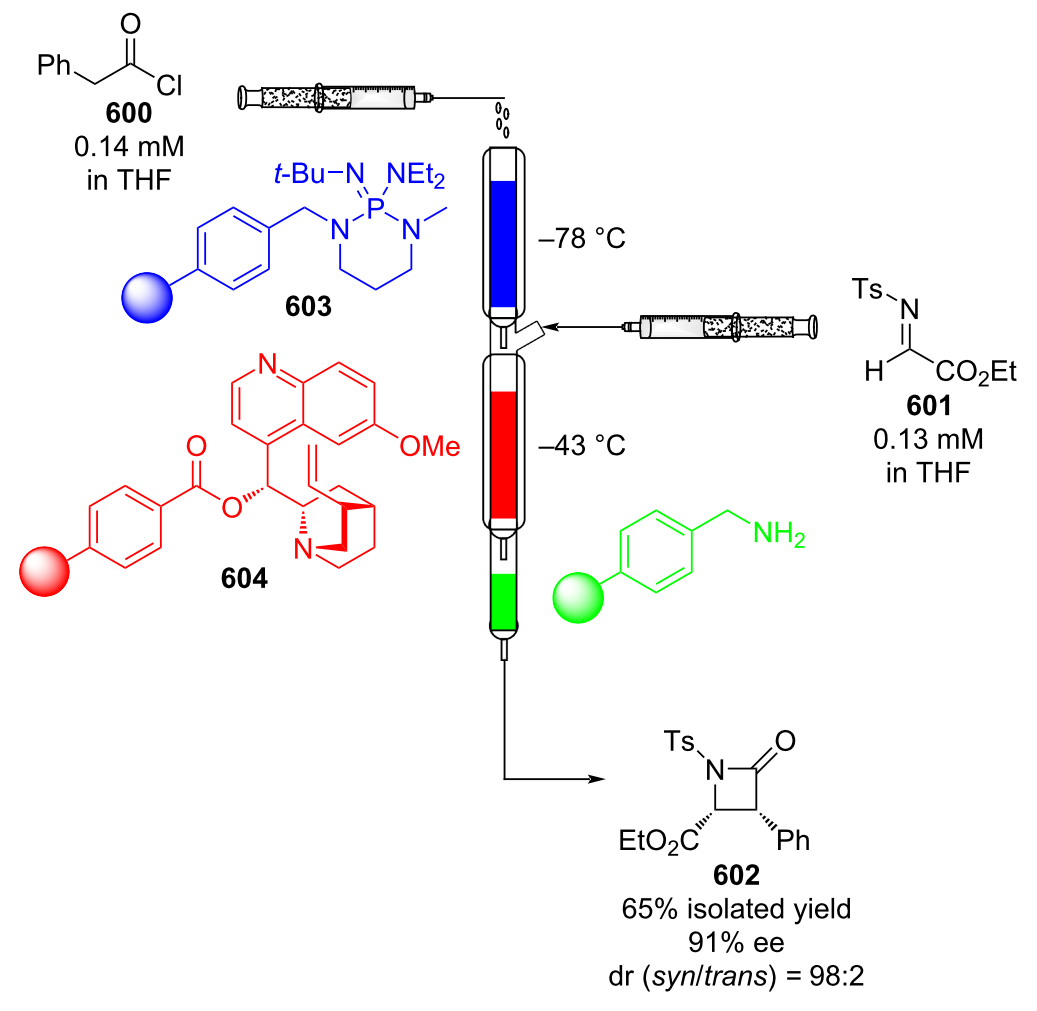

Scheme 136: Continuous synthesis of lactam 602 using glass column reactors.

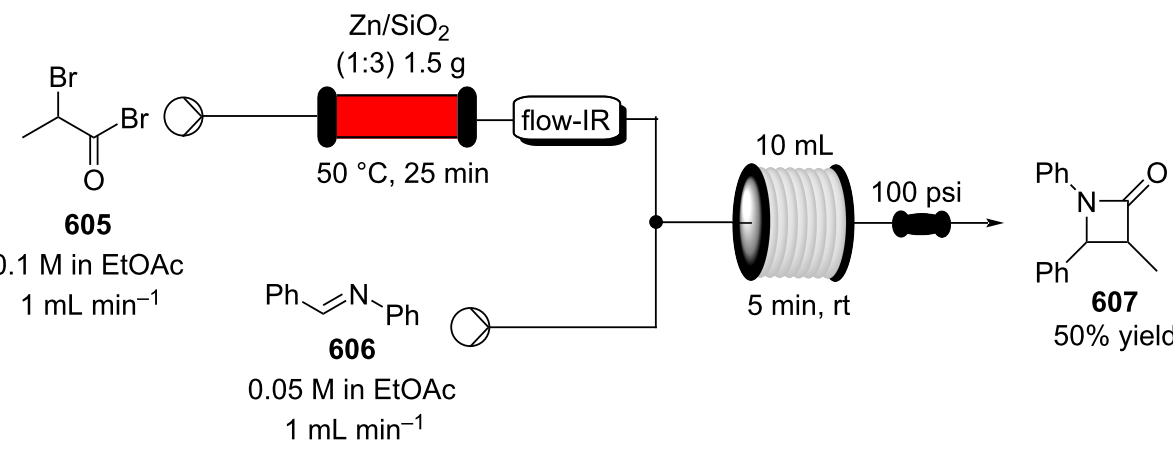

Scheme 137: In situ generation of ketenes for the Staudinger lactam synthesis developed by Ley and Hafner. 
trolled using an in-line flow IR spectrometer. Using this combination of apparatus, the lactam $\mathbf{6 0 7}$ was obtained after just 5 minutes in 50\% yield. Better results (98\% conversion) were obtained when diethyl ether was employed in a combined flowbatch mode. Due to high volatility of the ether solvent, the authors preferred to use an easier to handle solvent such as ethyl acetate.

Other continuous-flow systems in which a ketene was generated in situ and then directed into a [2+2] cycloaddition have been reported, many of these reactions present improved results compared to their batch modes with higher throughputs [522,523]

In a related approach a $[2+2+2]$ cyclotrimerization reaction was developed in flow. The flow mode was found particularly beneficial due to the harsh reaction conditions required and the ability to scale the reaction $[524,525]$. The system was based upon microwave irradiation instead of thermal heating, a glass coil was inserted into a standard microwave cavity of a Biotage reactor (Scheme 138) [525,526]. In this example, the product
609 was detected in $95 \%$ conversion within a 30 minutes residence time.

\section{Friedel-Crafts reactions}

Friedel-Crafts (FC) reactions have been exploited numerous times for the synthesis of F\&F compounds (Scheme 139). A few important examples include isocamphylcyclohexanol (216) from guaiacol (611) and camphene (610) [220], and the synthesis of cyclamenaldehyde (29) from cumene (613) [527]. Generally, musky odorants present a terpenoid-like skeleton which is usually obtained through a FC cyclisation as in the case of musk tetralin (619) prepared from $p$-cymene (616) and neohexene (617) $[528,529]$.

FC reactions typically require elevated temperatures and also Lewis acids such as aluminium trichloride in high stoichiometry, however, an enormous amount of waste salts are formed as byproducts. To prevent this waste, many heterogeneous catalysts have been investigated and described [530,531]. Flow chemistry has the potential to improve the reusability of these catalysts as has been previously discussed in this review (see<smiles>C#CCCCC#CCOCC#C</smiles>

608

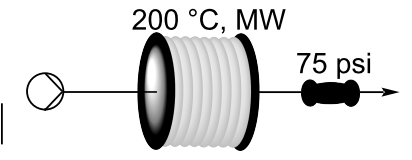

609

$95 \%$ conversion

Scheme 138: Application of $[2+2+2]$ cycloadditions in flow employed by Ley et al.
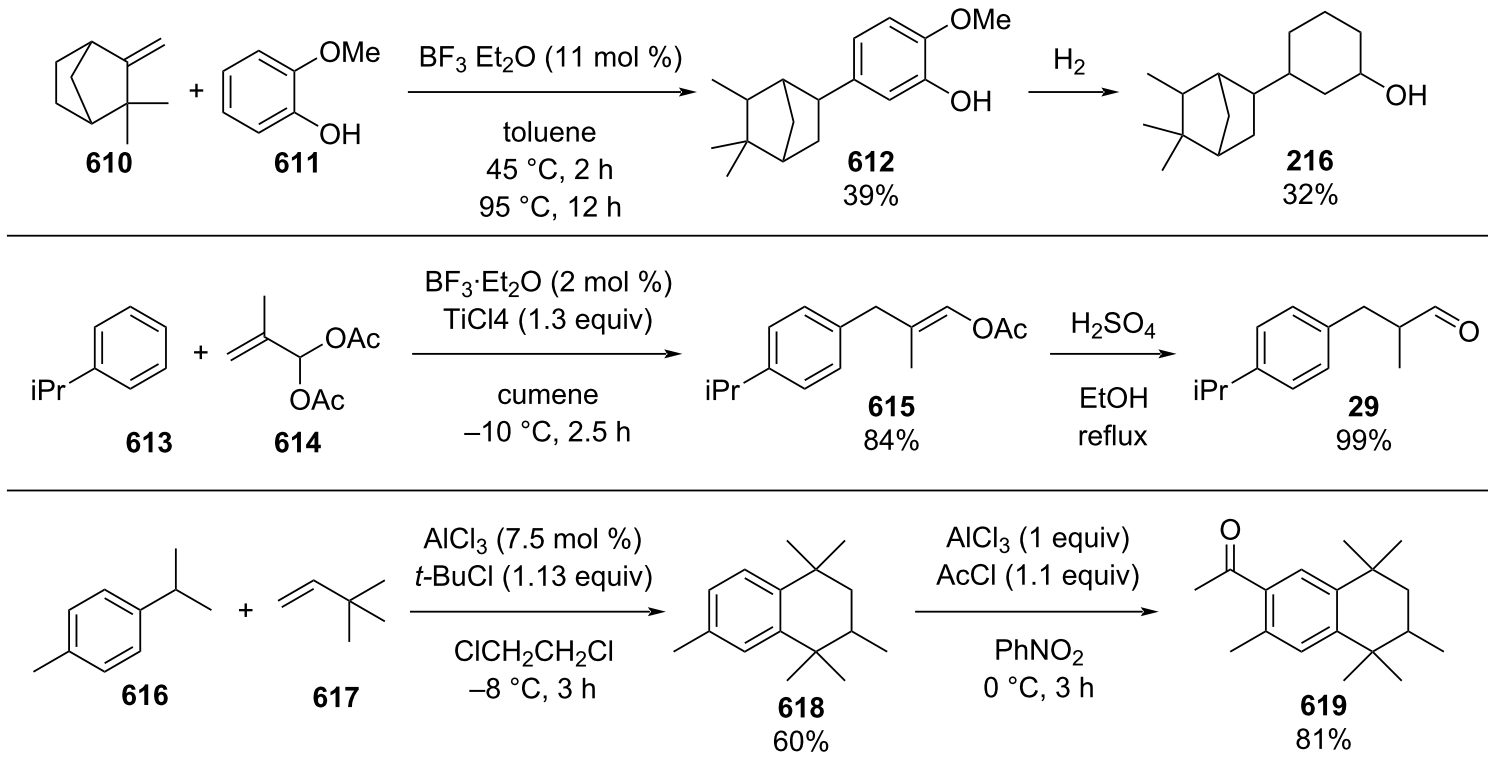
the chapters 'The advantages of a flow approach' and 'Condensation reactions'). One of the first papers describing FC reactions in a flow system came in 1977 from Toyoshima and Arata, where they optimised calcinated iron sulfate-catalysed FC reactions between toluene and isopropyl chloride in the gaseous phase [532]. As a first example, low conversions (10-20\%) were measured, however, selectivity and conversions remained steady over 50 minutes of runtime.

Over two decades later, Poliakoff et al. described a FC alkylation exploiting supercritical fluids such as $\mathrm{scCO}_{2}$ to achieve faster purification steps [533]. A sulfonic acid supported on polysiloxane (Deloxan ${ }^{\circledR}$ ) was employed as catalyst for the FC reaction of mesitylene with isopropanol. For this system, two different supercritical fluids ( $\mathrm{scCO}_{2}$ and scPropene) were investigated, and $\mathrm{scCO}_{2}$ proved more selective. The optimised flow apparatus allowed a modest yield of $290 \mathrm{mg} \mathrm{min}^{-1}$ of monoalkylated material in $19 \%$ yield.

In 2009, McQuade and co-workers achieved the FC reaction of isobutylbenzene (620) and propionic acid (621) in flow for the synthesis of ibuprofen (625, Scheme 140) [534].

For this setup, the authors chose to use triflic acid as the Lewis acid. Aluminium chloride proved to be more efficient, however, its byproducts were found detrimental for the subsequent steps. The neat stream of substrates $\mathbf{6 2 0}$ and $\mathbf{6 2 1}$ was mixed with triflic acid (622) in a tee junction cooled at $0{ }^{\circ} \mathrm{C}$ and then passed through a microreactor at $150{ }^{\circ} \mathrm{C}$. After 5 minutes, the precursor 627 (see structure of $\mathbf{6 2 7}$ in Scheme 141) was isolated in
$95 \%$. The overall synthesis of ibuprofen consisted of three microreactors; after the acylation step, a second coil at $50{ }^{\circ} \mathrm{C}$ was setup for the 1,2-aryl migration, and in the third warmed at $65^{\circ} \mathrm{C}$ where the methyl ester is hydrolysed to the final product 625.

More recently, an alternative continuous synthesis of ibuprofen (625) was reported by Jamison and Snead (Scheme 141) [535].

In this example, the aluminium chloride was solubilised in the propionyl chloride (626) and streamed through a coil reactor at $87^{\circ} \mathrm{C}$ along with the isobutylbenzene $(\mathbf{6 2 0})$. The flow stream was then quenched with $\mathrm{HCl}$ to terminate the reaction and avoid clogging of the insoluble aluminium salts. A separator membrane was then employed to separate the intermediate $\mathbf{6 2 7}$ from the aqueous waste. The organic phase containing 627 was collected in a separate flask from which it was then pumped and directly employed for the next steps to form ibuprofen. Additional flow processing of $\mathbf{6 2 7}$ afforded the ibuprofen sodium salt Na-625 in high yield (83\%), resulting in a short three-step continuous ibuprofen synthesis. The system allowed a production of $8.09 \mathrm{~g} \mathrm{~h}^{-1}$ of Na-625.

Intramolecular FC acylation using a microreactor setup allowed the synthesis of naphthalene derivative $\mathbf{6 2 9}$ in quantitative yield whilst also drastically reducing the reaction time (Scheme 142) [536]. Recently, microreactors have been used for direct acylation on naphthalene substrates. A solution of propionyl chloride (626, 1.3 equiv) and $\mathrm{AlCl}_{3}$ (1.5 equiv) in nitrobenzene was mixed at $0{ }^{\circ} \mathrm{C}$ with 2-methylnaphthalene and then streamed

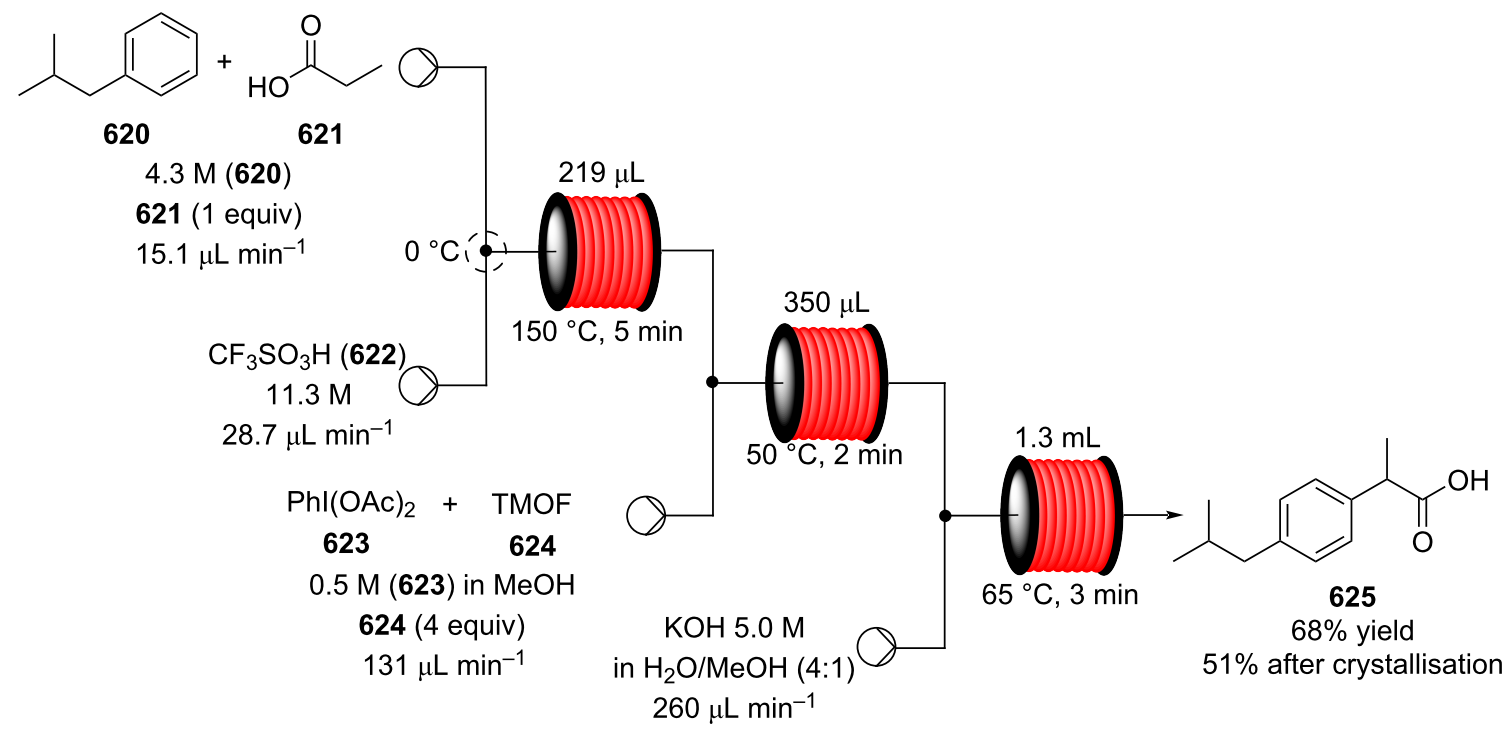



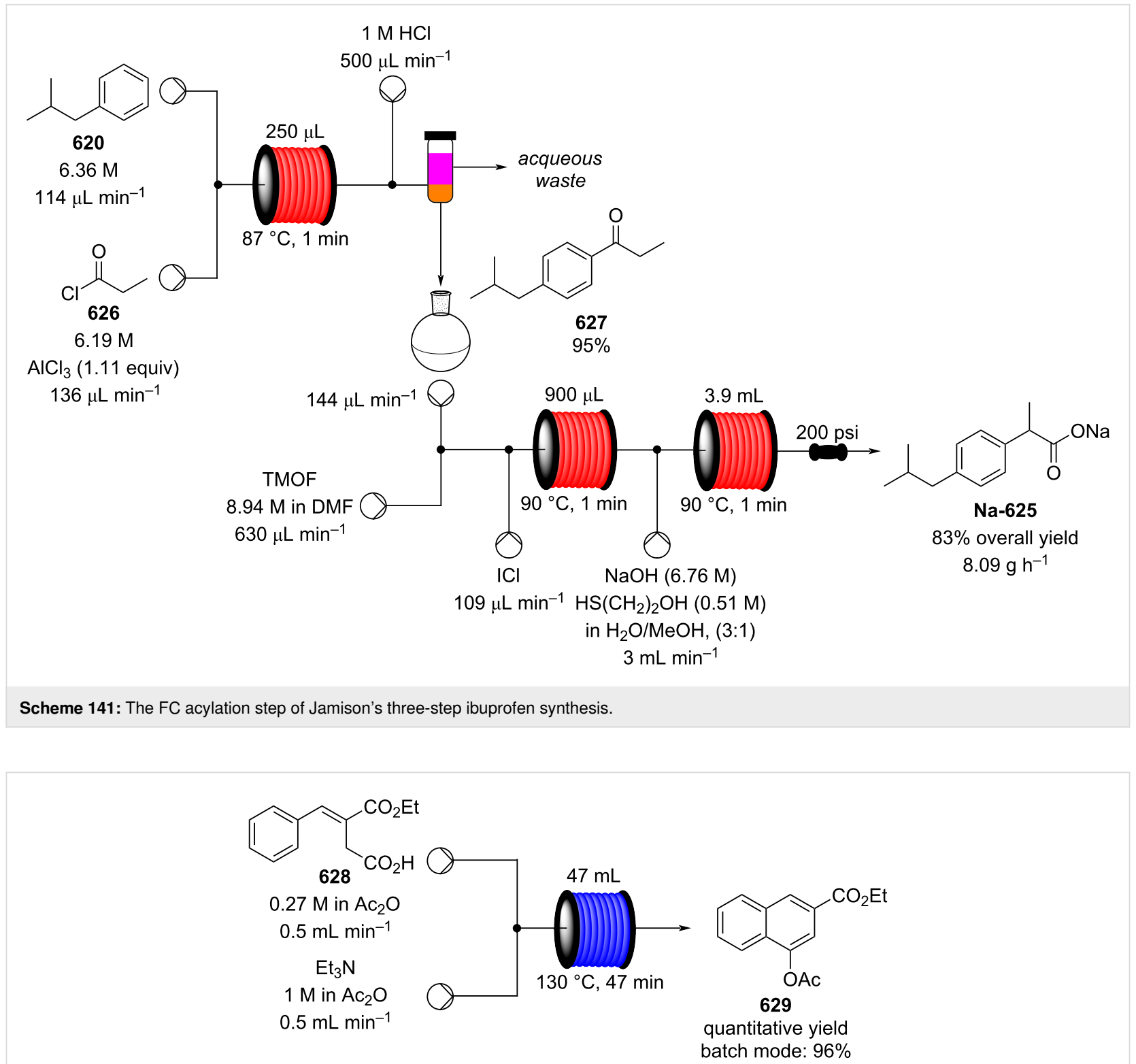

Scheme 142: Synthesis of naphthalene derivative 629 via FC acylation in microreactors.

through a micro-channelled reactor at $40{ }^{\circ} \mathrm{C}$ for 60 minutes. The authors claimed high selectivity $(87.5 \%)$ and yield $(85.8 \%)$ [537].

Finding the optimal reaction conditions remains a time and resource-consuming process. Flow chemistry techniques can expedite these investigations, drastically reducing the resource required during the process optimisation stages. A rapid catalyst screening system was developed by Weber, Floreancig and co-workers employing a system of loop injectors connected to a UHPLC (ultra-high performance liquid chromatography) system [538]. The loop injector creates individualised reaction zones where different catalysts can be screened (Scheme 143).
The authors explained no mixing between reaction zones occurs due to the narrow diameter of the capillaries and the low flow velocity, which suppress radial diffusion of reagents. At a flow rate of $0.9 \mu \mathrm{L} \mathrm{min}{ }^{-1}$, roughly one catalyst per hour could be sequentially screened (it should be noted that parallel systems could be run). The reactor was applied to the intramolecular FC acylation of acylaminal 630 to the $N$-acyl amine 631. The authors found that $20 \mathrm{~mol} \%$ of $\operatorname{Er}(\mathrm{OTf})_{3}$ at $40{ }^{\circ} \mathrm{C}$ for 30 minutes was optimal furnishing $93 \%$ yield. When the catalyst was employed in the related batch reaction, the system down-performed, requiring an increase in the amount of catalyst ( 1 equivalent) and a prolonged reaction time (8 hours). 


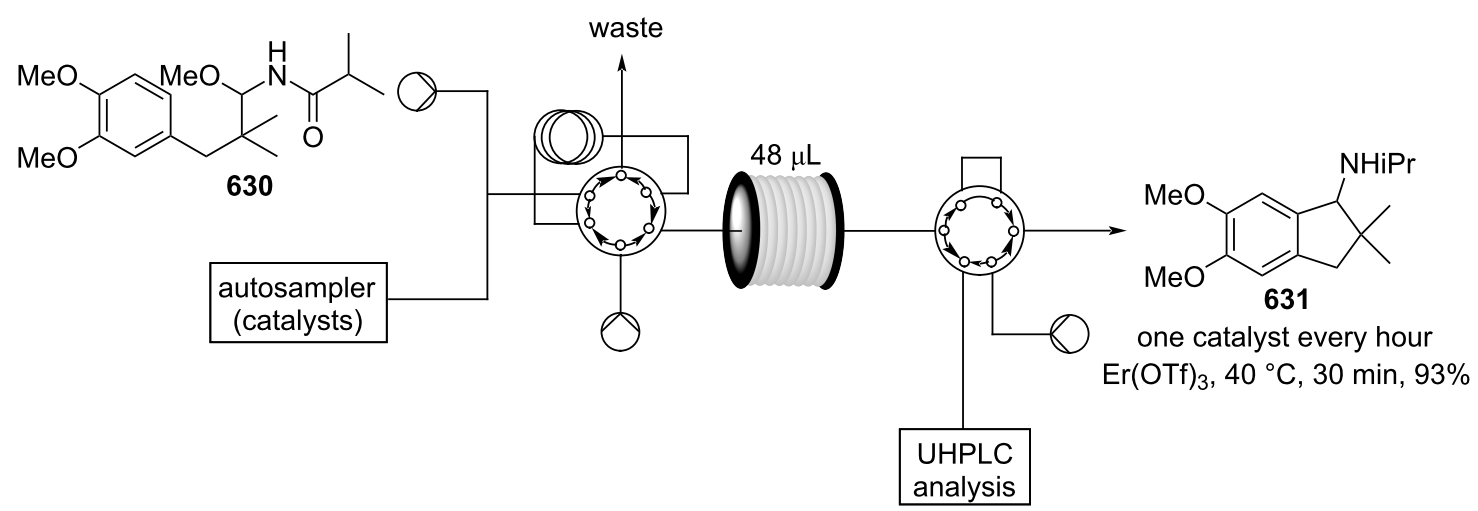

Scheme 143: Flow system for rapid screening of catalysts and reaction conditions developed by Weber et al.

Similarly, Bourne, Muller and co-workers developed a multistep kinetic model for use with flow conditions [539]. Indeed, flow chemistry has enhanced the ability of organic chemists to study and develop kinetic models of reactions creating reproducible conditions for scale-up. In their study the authors investigated the formation of the pharmaceutical intermediate $\mathbf{6 3 4}$ starting from 2,4-dichloropyrimidine (632) and 1-methylindole (633). This FC reaction can also lead to other byproducts such as 635 and the dimer $\mathbf{6 3 6}$. The flow apparatus consists of two HPLC pumps connected via a T-piece to a temperature regulated tubular coil reactor. In order to maximise the yield and reduce the byproduct formation, they developed a kinetic model employing a definitive screening design (DSD), augmenting the level of information acquired and reducing the number of experimental runs. The simulation determined the optimal conditions (Scheme 144) where the lowest amount of byproducts $\mathbf{6 3 5}$ (7\%) and $\mathbf{6 3 6}$ (trace) was formed. These results were comparable to the batch optimisation process $(82 \%$ yield).
In 2019, Oh and co-workers developed a flow system for the $\mathrm{AlCl}_{3}$-catalysed $\mathrm{FC}$ reaction of acyl chlorides to alkynes to yield $\beta$-chlorovinyl ketones [540]. The reaction in batch mode provided mixtures of the $Z$ - and $E$-isomers due to a $\mathrm{AlCl}_{3}$ alkyne complex formation, which promotes isomerisation. The authors found that by reducing the reaction times this process was decreased, therefore a mixing chip (MiChS $\beta$-type) with a secondary residence time loop $(100 \mu \mathrm{L})$ used for the task (Scheme 145).

A $0.1 \mathrm{M}$ solution of the alkyne in dichloromethane was mixed with a solution of the acyl chloride $\mathbf{4 6 0}$ and $\mathrm{AlCl}_{3}$ in the mixing chip at $23{ }^{\circ} \mathrm{C}$ and then sent to the loop reactor for 3 seconds. The output stream was immediately quenched in a conical flask with water. The $\beta$-chlorovinyl ketone $\mathbf{6 3 8}$ was obtained in $82 \%$ yield, with a 5:1 $E / Z$ ratio. The substrate was later employed in a flow preparation of isoxazoles through the reaction with vinyl azides [541].

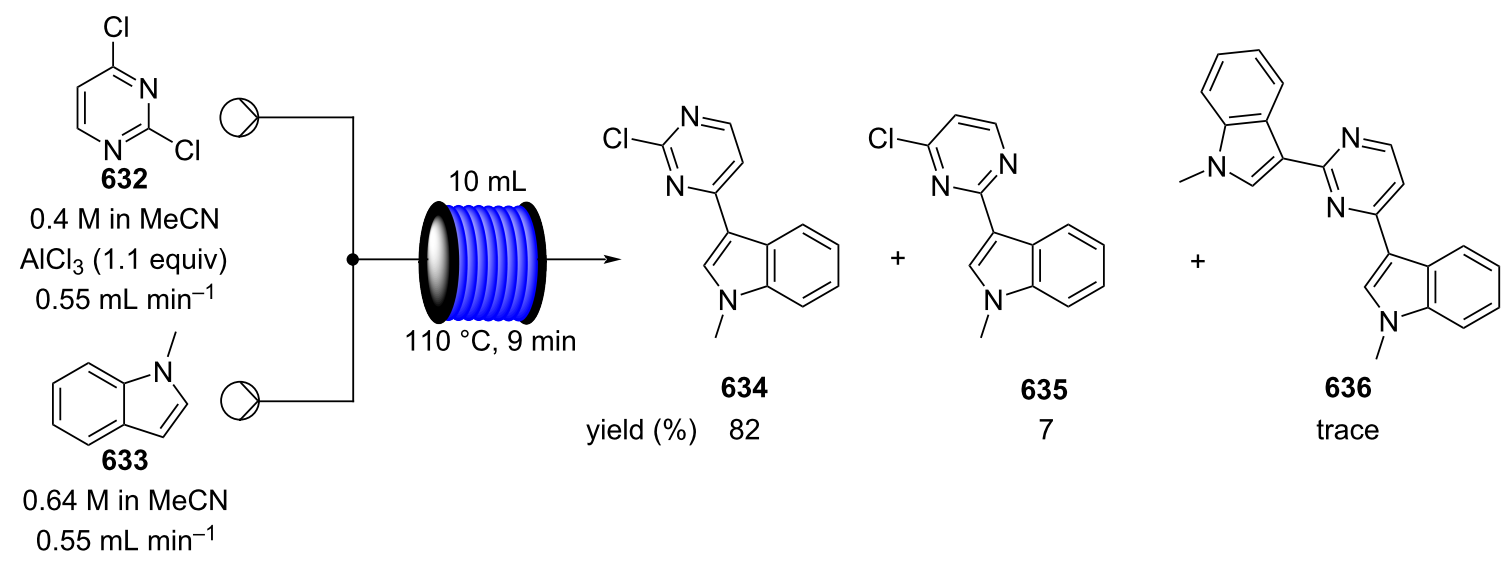

Scheme 144: Continuous-flow system developed by Buorne, Muller et al. for DSD optimisation of the FC acylation to 634. 


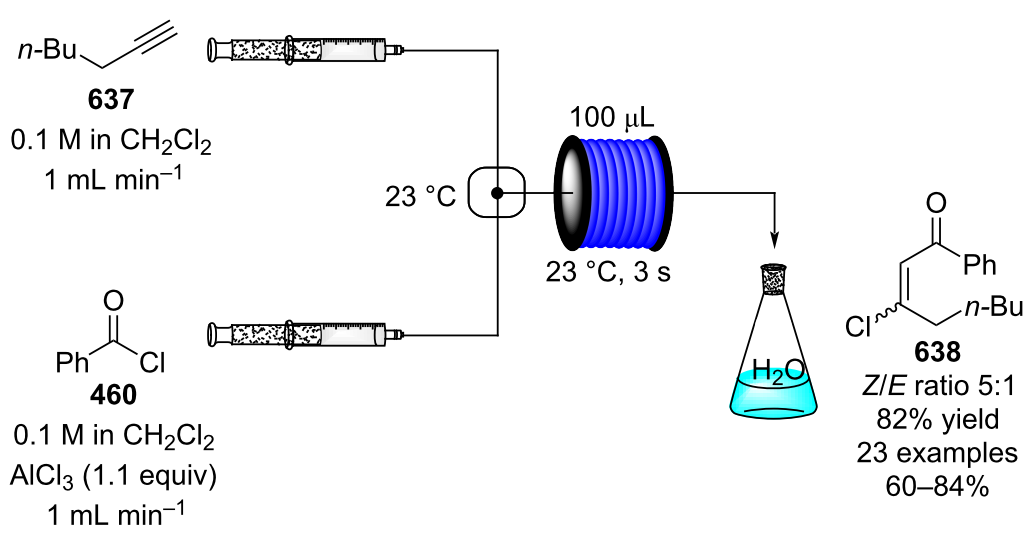

Scheme 145: Continuous-flow FC acylation of alkynes to yield $\beta$-chlorovinyl ketones such as 638 .

Very few examples of flow conditions applied to F\&F chemicals have recently been reported. However, as previously highlighted, the FC acylation is thought of paramount importance to the industry. Recently, Wang and co-workers developed a flow system for the preparation of tonalide (619), from 1,1,3,4,4,6hexamethyltetralin (618) [542]. A $2.5 \mathrm{M}$ stream of 618 in 1,2dichloroethane was mixed with acetyl chloride (639) and the catalyst in a $\mathrm{T}$-shaped mixer and through a microchannel module at $-5{ }^{\circ} \mathrm{C}$ (Scheme 146). The reaction mixture was then quenched with an aqueous solution of sodium chloride. The system allowed formation of $\mathbf{6 1 9}$ in $97.3 \%$ yield, which resulted in a more efficient system then the classic batch mode (95.3\%).

Heterogeneous catalysis for FC acylation is still a hard task to achieve due to rapid catalyst deactivation attributed to the adsorption of either byproducts or reactants onto the active sites, bringing about a sudden decrease in the catalytic activity. Aribert and co-workers developed a continuous-flow system employing zeolite $\mathrm{Y}$ as the catalyst. After a single use, the yield dropped drastically below $20 \%$ from $80 \%$, and calcination is required to re-establish the activity [543]. In total twelve byproducts along with other heavy molecules ("coke") were found by Zeng et al. on the surface of an HBEA zeolite (acidic form of the $\beta$-zeolite polymorph A) when exploited for continuous FC acetylation of toluene. The authors noticed an enhancement in activity using an excess of toluene and acetic acid as an additive [544]. A decrease of the zeolite's stability was also described by Kobayashi et al. in 2019 [545]. The authors noticed a reduction of aluminium content in the zeolite structure, which inevitably brought about a decrease in activity from $94 \%$ down to $59 \%$ yield $(\mathbf{6 4 0} \rightarrow \mathbf{2 9 0})$ after 28 hours of usage. To their delight, they found that doping the $\beta$-zeolite with zirconium cations increased the catalyst stability. The flow system was tested on up to 20 molecules, gaining the acylated materials in $70-99 \%$ yield (Scheme 147). The catalyst could be used for up to 5 days without any substantial loss of activity. The flow system employed either chlorobenzene or the arene substrates themselves as the solvent.

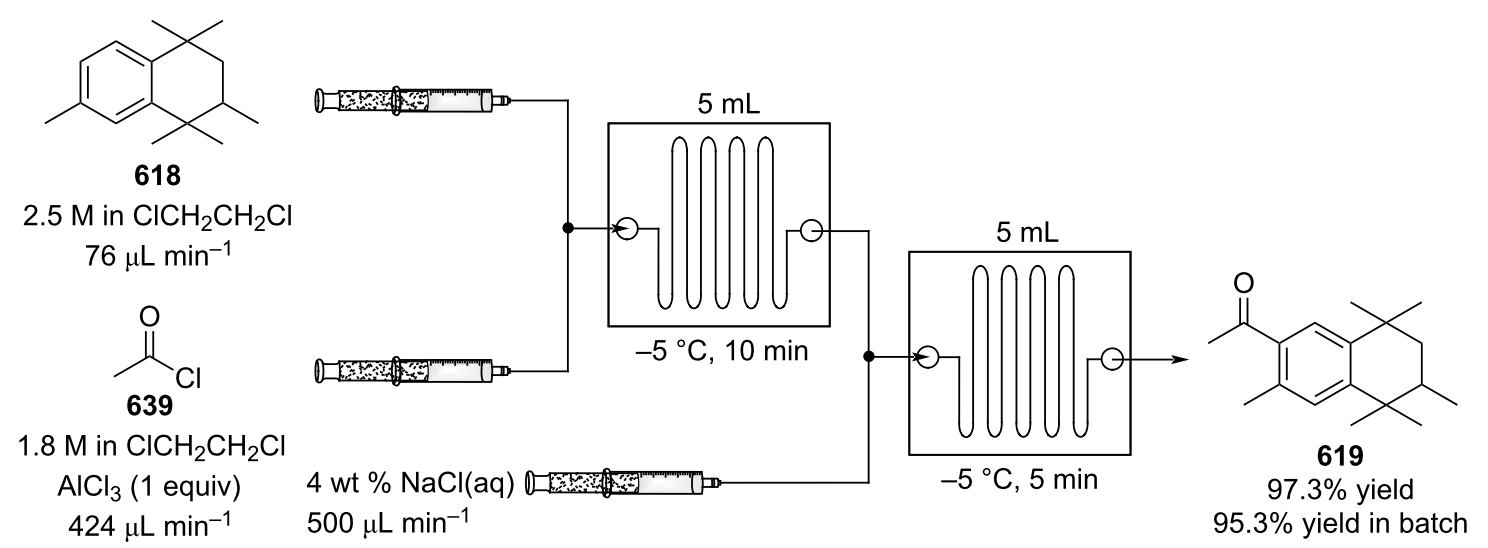

Scheme 146: Continuous-flow synthesis of tonalide (619) developed by Wang et al. 


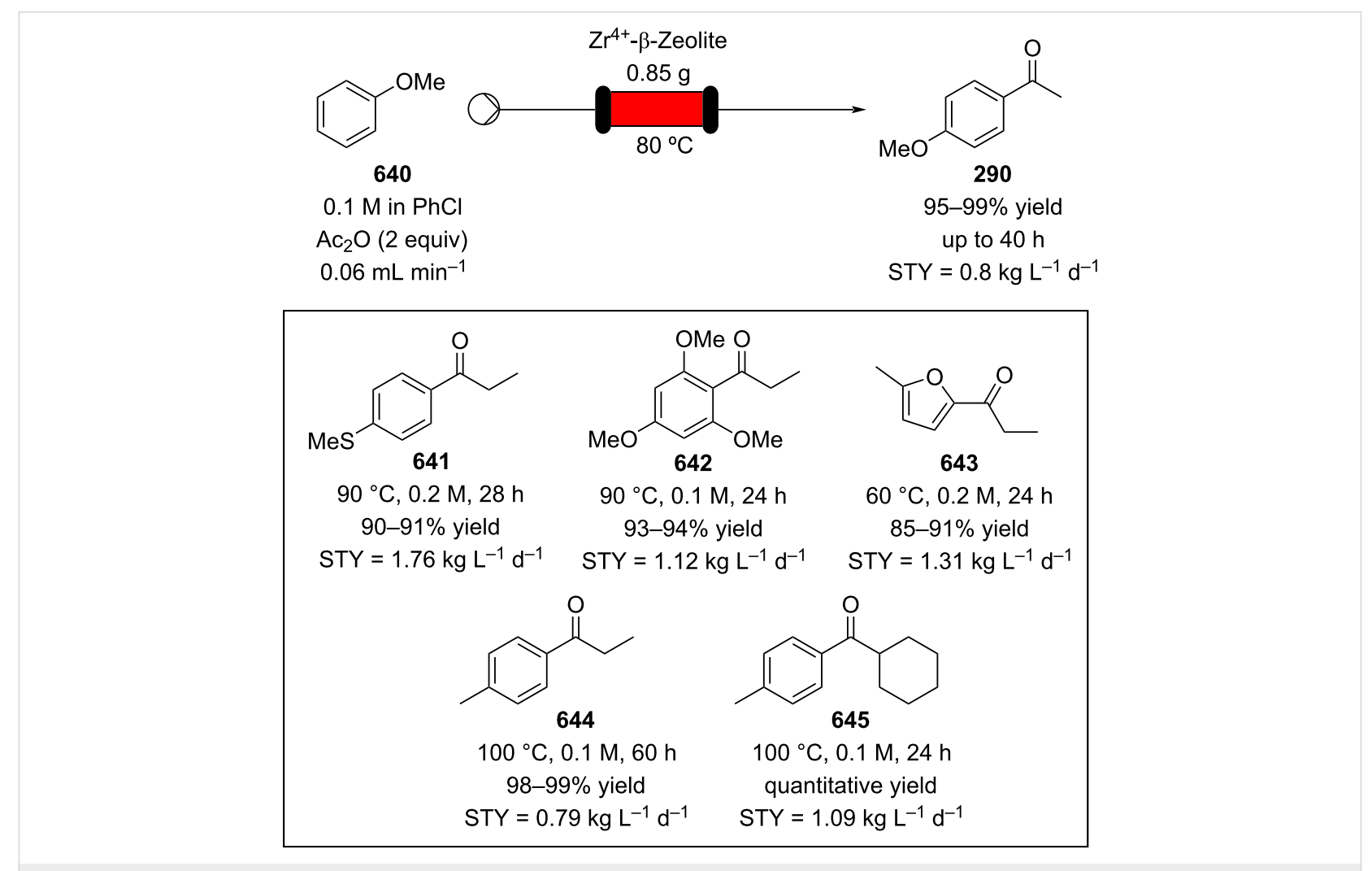

Scheme 147: Continuous-flow preparation of acylated arene such as 290 employing $\mathrm{Zr}^{4+}-\beta$-zeolite developed by Kobayashi et al.

Heterogeneous catalysts other than zeolites have also been described lately, such as the $\alpha-\mathrm{Fe}_{2} \mathrm{O}_{3}$ nanoparticles modified by Guo et al. [546], and the metal organic framework (MOF) with zirconium oxides on silica gel developed by Lin and co-workers [547]. These systems can be employed successfully for up to 4 hours prior to loss of activity in a flow reactor.

An area which is starting to gain much more interest is the use of immobilised asymmetric catalysts. A highly robust packedbed column reactor containing a supported phosphoric acid catalyst for enantioselective catalysis of FC reactions involving indoles [548] in flow was reported in 2014 [549]. Recently, a hydroquinine-based polymer supported catalyst 648 has also been developed by the same group for the continuous Aza-FC reaction of $\alpha$-naphthol (647) with 2-amino-2-oxindole derivative 646 [550]. The flow system was allowed to run over 400 minutes to yield the product $\mathbf{6 4 9}$ in $80 \%$ yield and $94 \%$ ee (Scheme 148). The authors pointed out the reduce reaction times (10.3 min vs $3 \mathrm{~h}$ ) compared to the batch mode and a productivity of 649 of $2.2 \mathrm{mmol} \mathrm{mmol}_{\text {cat }}{ }^{-1} \mathrm{~h}^{-1}$.

This area is still relatively underdeveloped due to the complexity and challenges inherent in generating asymmetric catalysts on solid support but will evidently become of greater importance.

\section{Hydroformylation}

Hydroformylation is a powerful and atom-efficient technique used for the introduction of formyl groups by conversion of alkene functions, often with high selectivity. Aldehydes are among the most important groups of molecules to the F\&F industry as both starting materials and also as products. It is reported that 2-methyldecanal and 2-methylundecanal can be prepared from 1-decene (366) via a hydroformylation process [551]. As with the other reaction types that require a gaseous reagent supply, flow chemistry presents several advantages for reasons already discussed, such as safety, ease of gas delivery and control over reagent exposure times and stoichiometry.

In one specific reaction, Cole-Hamilton et al. employed $\mathrm{scCO}_{2}$ and ionic liquids to increase catalyst surface contact and reduce deactivation (Scheme 149) [552].

The catalyst was dissolved in 1-pentyl-3-methylimidazolium 3-(diphenylphosphaneyl)benzenesulfonate, [PnMIM][TPPMS] (651), to which a 1-octene (650) solution in the $\mathrm{scCO}_{2}$ was flushed through. The flow system allowed preparation of a 3:1 linear/branched $(\mathbf{3 4 5} / \mathbf{6 5 2})$ mixture of aldehyde with a space time yield of $0.2 \mathrm{~mol} \mathrm{~L}^{-1} \mathrm{~d}^{-1}$. However, low substrate flow rate could only be used as increasing the flow rate would result in overfilling of the reactor and leaching of the ionic liquid. The 


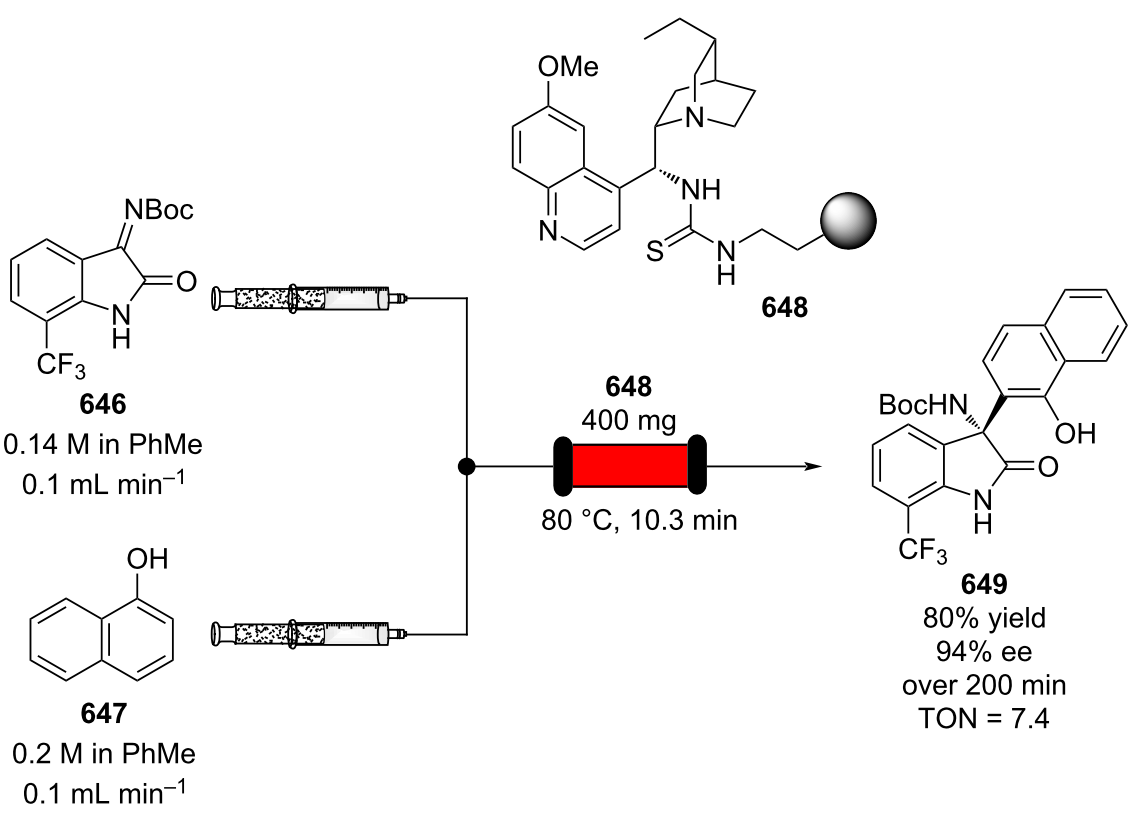

Scheme 148: Flow system applied on an Aza-FC reaction catalysed by the thiourea catalyst 648 .

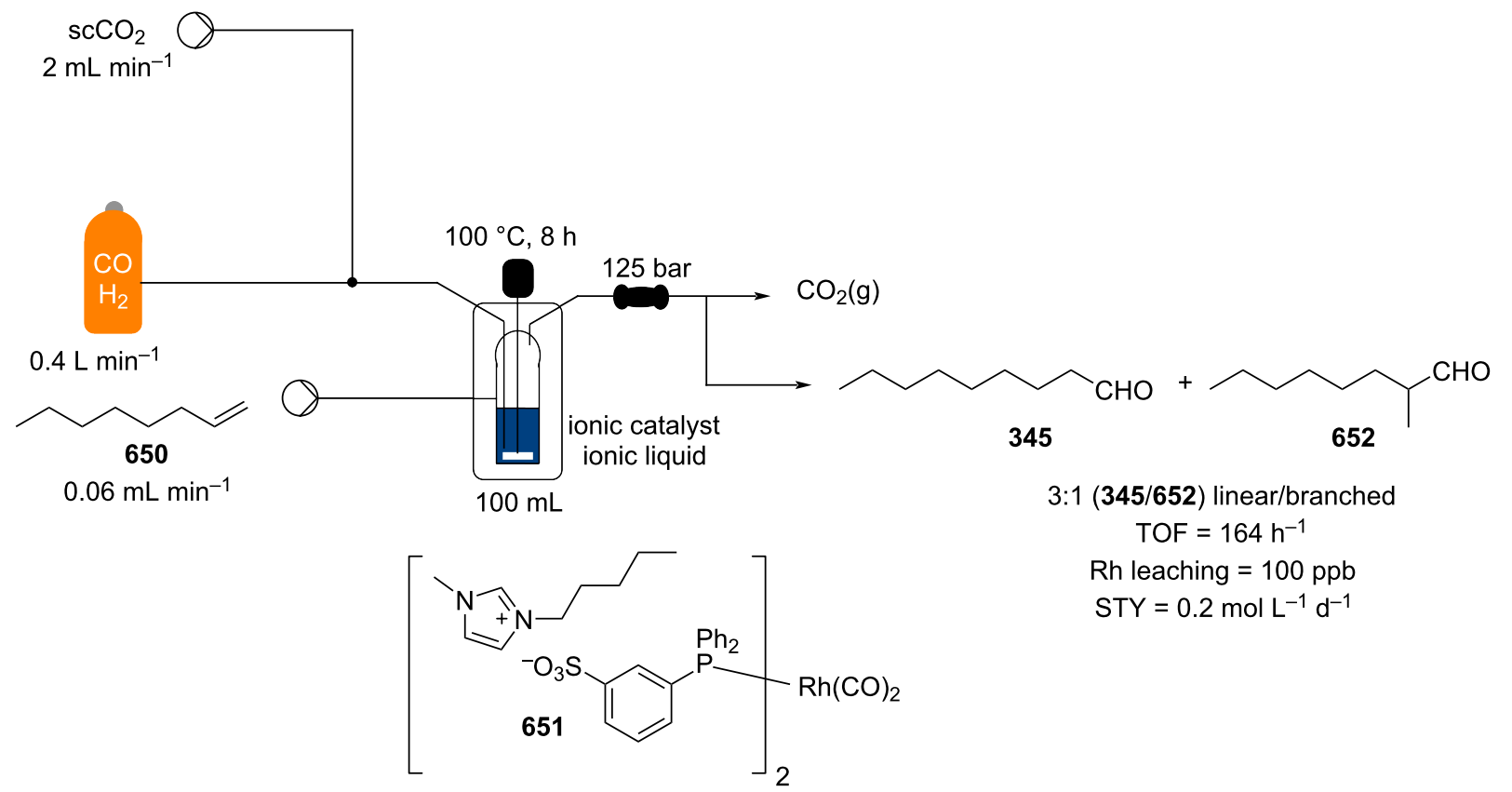

Scheme 149: Continuous hydroformylation in $\mathrm{scCO}_{2}$.

system performed at a lower efficiency than the apparatus described by the same group which used a higher pressure (200 bar vs 125 bar) [553]. Continuous-flow hydroformylation processes performed under supercritical conditions and employing heterogeneous catalysts have been comprehensively reviewed recently [554].
To recycle the secondary phase catalyst, supported ionic liquid phase (SILP) technology was also exploited. In 2019, Haumann et al. developed a membrane-based heterogenous catalyst made of a composite material of multiwalled carbon nanotubes modified with silica particles to control the porosity [555]. The composite supported a rhodium catalyst which avoided deactivation 
due to accumulation of high boiling point cross aldol-derived products. The continuous-flow system employed for the gasphase hydroformylation of 1-butene gave high linear to branched selectivity (96\%) and $64 \%$ selectivity towards the $n$-pentanal product. The apparatus allowed a large reduction in byproducts derived from secondary aldol condensation.

In 2011, Ley and co-workers developed a flow reactor for the hydroformylation of styrenes based upon gas addition using a tube-in-tube reactor system [556]. The reactor was highly efficient (81-97\% conversion) and flexible (12 examples). As a proof-of-concept, the synthesis was further extended to incorporate an initial Heck reaction between $p$-iodoanisole $(\mathbf{6 5 3})$ and ethylene gas using JohnPhos and a second tube-in-tube reactor preparing styrene $\mathbf{6 5 4}$ which was progressed to aldehyde $\mathbf{6 5 5}$ (Scheme 150). The first stage Heck reaction was found to generate significant amounts of palladium black, however, this was easily removed using a cartridge filled with cotton wool to act as a filter preventing downsteam aggrgation and blockage of the reactor
Takahashi et al. assembled a simple syringe pump driven system attached to a stainless steel coil which was employed for the hydroformylation of 1-octene (650) [557]. The system only gave moderate $\approx 19 \%$ conversions, however, the authors suggested the unreacted alkene could be easily recycled. This simple unit does highlight how to safely handle hazardous reagents such as syngas in a labortary employing a flow reactor.

Studying the kinetics of a process is paramount to its optimisation. The Jensen and Abolhasani groups independently developed two microreactor systems which allow kinetic measurements alongside investigation of optimum reaction conditions. Jensen's apparatus consisted of a $220 \mu \mathrm{L}$-silicon microreactor wherein the solution of catalyst and ligand was mixed with the syngas and 1-octene $(\mathbf{6 5 0})$. The liquid outlet stream was then separated from the gas phase and analysed with GC and ATRFTIR [558]. Abolhasani's system was fully automated and allowed on-demand screening of several mixtures of catalysts and ligands as well as the reaction conditions [559]. The setup employed an horseshoe-shaped tube-in-tube reactor wherein the

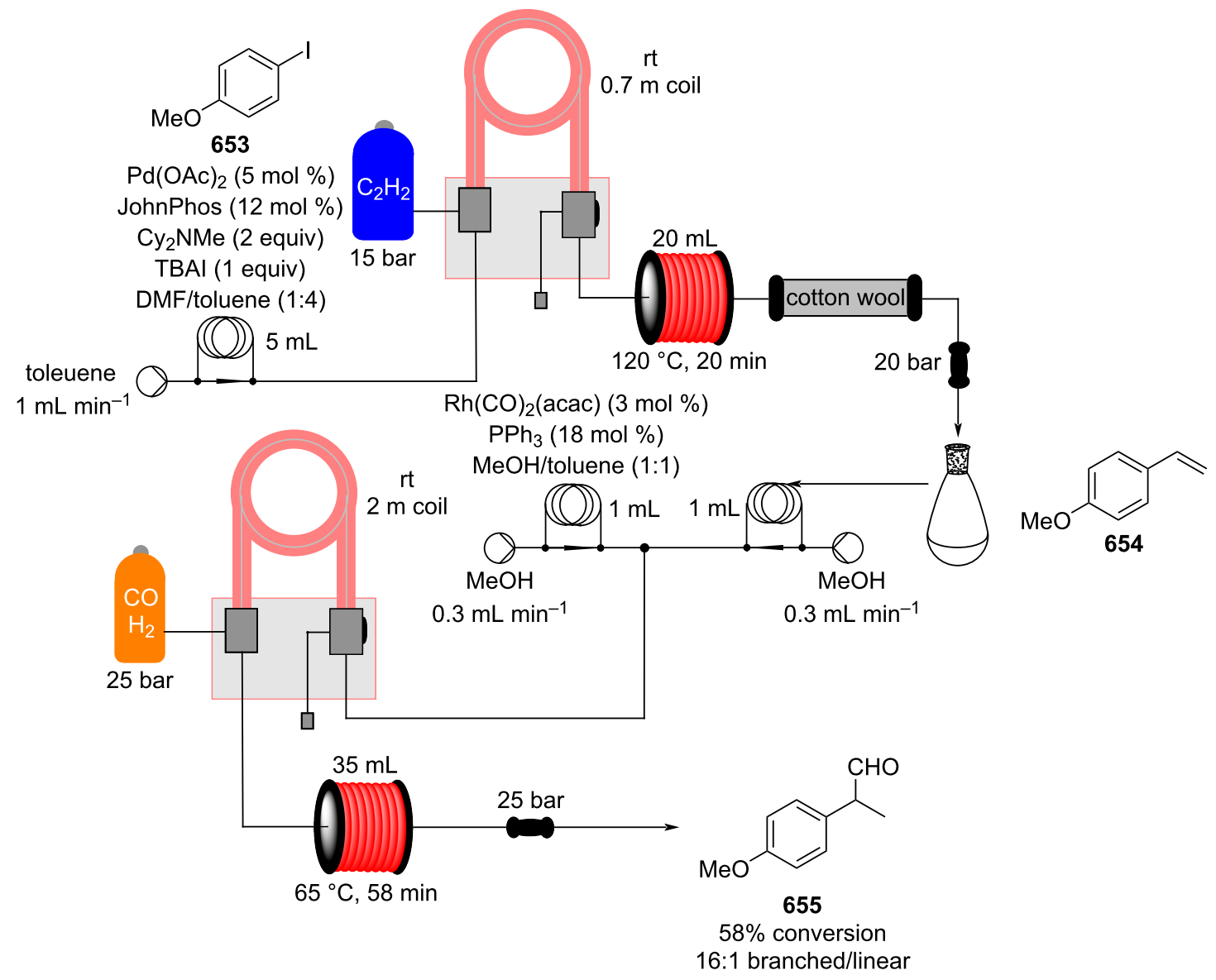

Scheme 150: Two-step flow synthesis of aldehyde 655 through a sequential Heck reaction and subsequent hydroformylation. 
reaction mixture droplet oscillates reacting with the syngas coming from the outmost tube (Scheme 151).

The authors pointed out the possibility of performing experiments exploiting a wide range of reaction times (10 seconds to 1 day) without changing internal volume reactor or flow rates. The outlet was then mixed with a quenching solution and analysed via a HPLC system coupled with evaporative light scattering detector (ELSD) and MS. The optimal conditions yielded by this screening platform was then applied to the synthesis of nonal in high regioselectivity (linear/branched, L/B > 15) under low syngas pressures (3.4 bar, Scheme 151) [560].

Sundmacher and co-workers developed a miniplant setup for the continuous hydroformylation of 1-dodecene (656) in a thermomorphic multiphase system (Scheme 152) [561].
To recycle the catalyst, a $N, N$-dimethyformamide (DMF)/ $n$-decane solvent system was employed which forms an homogenous phase at high temparatures around $95-115{ }^{\circ} \mathrm{C}$ and two separate phases when cooled down. The homogeneous reaction was initially measured and modelled on three different reactor systems: continuous stirred tanks reactor (CSTR), plug flow reactor (PFR) coupled with CSTR, and semibatch reactor (SBR) coupled with CSTR. Comparing all three cases, the last two allowed longer residence times and higher conversion, reducing the need of recycling the reagent. Furthermore, the PFR-CSTR system was experimentally constructed and configured [562]. A solution of 1 -dodecene (656) in $n$-decane was mixed with the rhodium catalyst and the ligand (BiPhePhos) in DMF and then the biphasic system was streamed through the PFR. The PFR consisted of a helically coiled reactor with two different temperature zones $\left(95-115^{\circ} \mathrm{C}\right)$ which allowed for better reaction kinetic control than a single temperature. The first part was

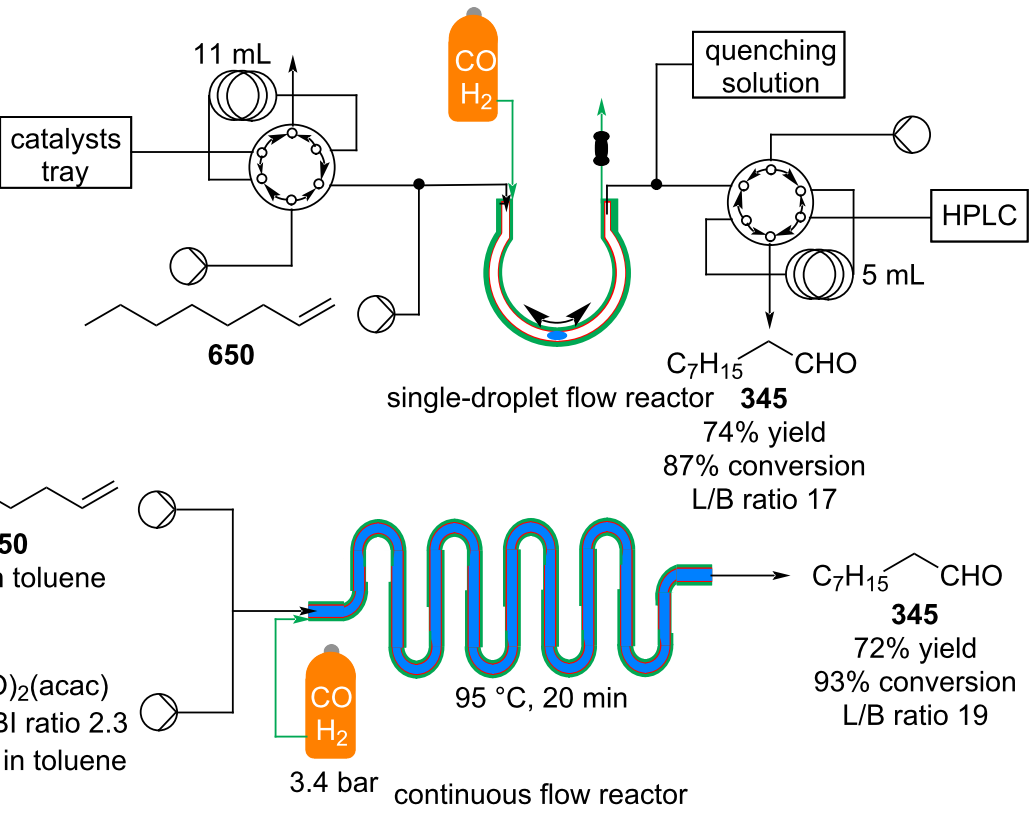

Scheme 151: Single-droplet (above) and continuous (below) flow reactors developed by Abolhasani et al. for the process optimisation of hydroformylation reactions.

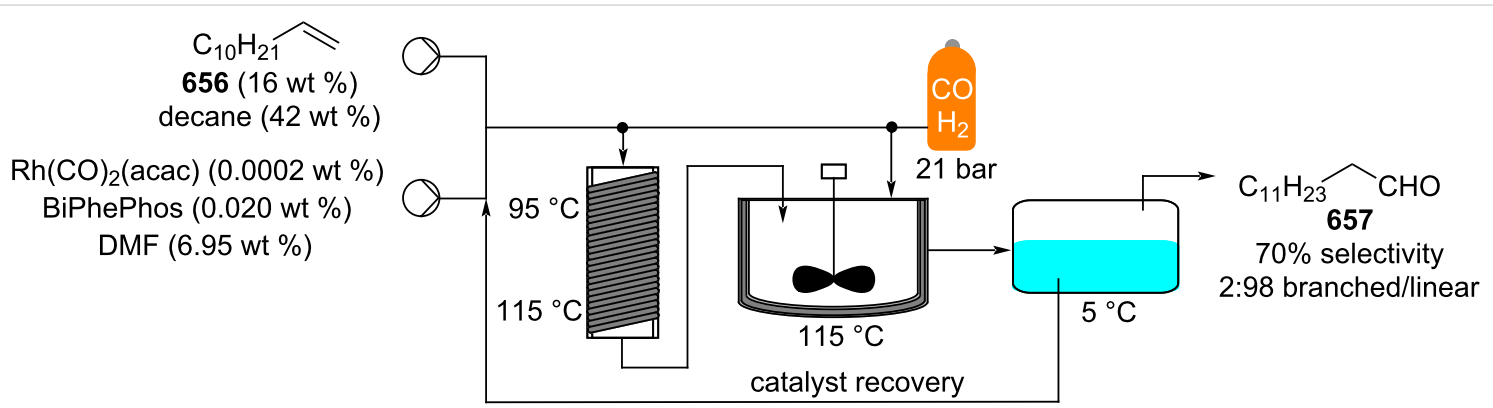

Scheme 152: Continuous hydroformylation of 1-dodecene (655) using a PFR-CSTR system developed by Sundmacher et al. 
heated at $95{ }^{\circ} \mathrm{C}$, which allowed the hydroformylation rate to be enhanced and the isomerisation rate to be reduced. The final section of the coil was warmed up to $115{ }^{\circ} \mathrm{C}$ and the outlet stream was then transfered into a CSTR (also heated at $115^{\circ} \mathrm{C}$ ) where the hydrogen content was higher. In this reactor, the fraction of isomer formed is allowed to be re-isomerised creating more reagent for hydroformylation The system allowed the tridecanal (98:2 linear/breanched) to form in 70\% selectivity and full conversion, with a low rhodium leaching content of between 1.56 and $2.26 \mathrm{ppm}$.

Recently, Zhang et al. developed a nature-inspired microreactor which is capable of increasing the gas-liquid mixing [563]. The reactor consists of an honeycombed microchannel reactor in which the channel cappilaries and branches assist with breaking the gas slugs up, enhancing the contact area in the mixture. With such a flow apparatus, the hydroformylation of 1-hexene occurs at $90 \%$ yield after only 30 minutes residence time compared with the over 3 hours required in batch mode. In addition, the regioselectivty of the reaction $(\mathrm{L} / \mathrm{B})$ was also enhanced to 24.7 from 16.9 obtained in batch.

In furtherance of this research asymmetic hydroformylations (AHF) have also been investigated in flow. Enantioselecitivity and regioselectivity have been reported to be highly sensitive to changes in CO partial pressure [564]. For this reason, a better gas-liquid mixing as provided in flow would be expected to impact the transformation. In 2016, Landis et al. at Eli Lilly \& Co. developed a reactor capable of extremely high mass transfer rates but coupled with long residence times. The reactor consists of a $17.8 \mathrm{~mL}$ stainless steel coiled reactor with a small internal diameter (i.d. $0.56 \mathrm{~mm}$ ) where every 3.66 metres a $0.3 \mathrm{~mL}$ vertical pipe was placed [31]. With this setup, the vertical pipes would be principally filled up with liquid and the tubing mostly filled with vapour (90-93\%) so that the residence times can be longer without influencing the amount of gas present along the coil reactor. Using this reactor setup, the AHF of 2-vinyl-6-methoxynaphthalene (658) was carried out employing the catalyst $\mathrm{Rh}$ (acac)(BDP) (659) at a loading of $0.074 \mathrm{~mol} \%$ at high pressure (150-800 psi). A $0.502 \mathrm{M}$ solution of substrate $\mathbf{6 5 8}$ along with the catalyst in toluene was mixed with the syngas flow and pumped through the stainless steel reactor heated up to $70{ }^{\circ} \mathrm{C}$ (Scheme 153). The flow system ran uninterrupted for over 130 hours yielding $\mathbf{6 6 0}$ in high conversion (97-98\%), high regioselectivity $($ branched/linear = 13-27), and enantioselectivity (ee $=80-92 \%$ ) [32].

In 2019, a supported catalyst for AHF was developed by Garcia-Suarez, Godard and co-workers [565]. The employed ligands consists of 1,3-diphosphites with furanose backbone carrying a pyrene functional group capable of $\pi-\pi$ stacking interactions with hydrophobic supports. The catalyst was then immobilised on several carbonic supports such as MWNT, reduced graphene oxide (rGO), and carbon beads (CBs). After
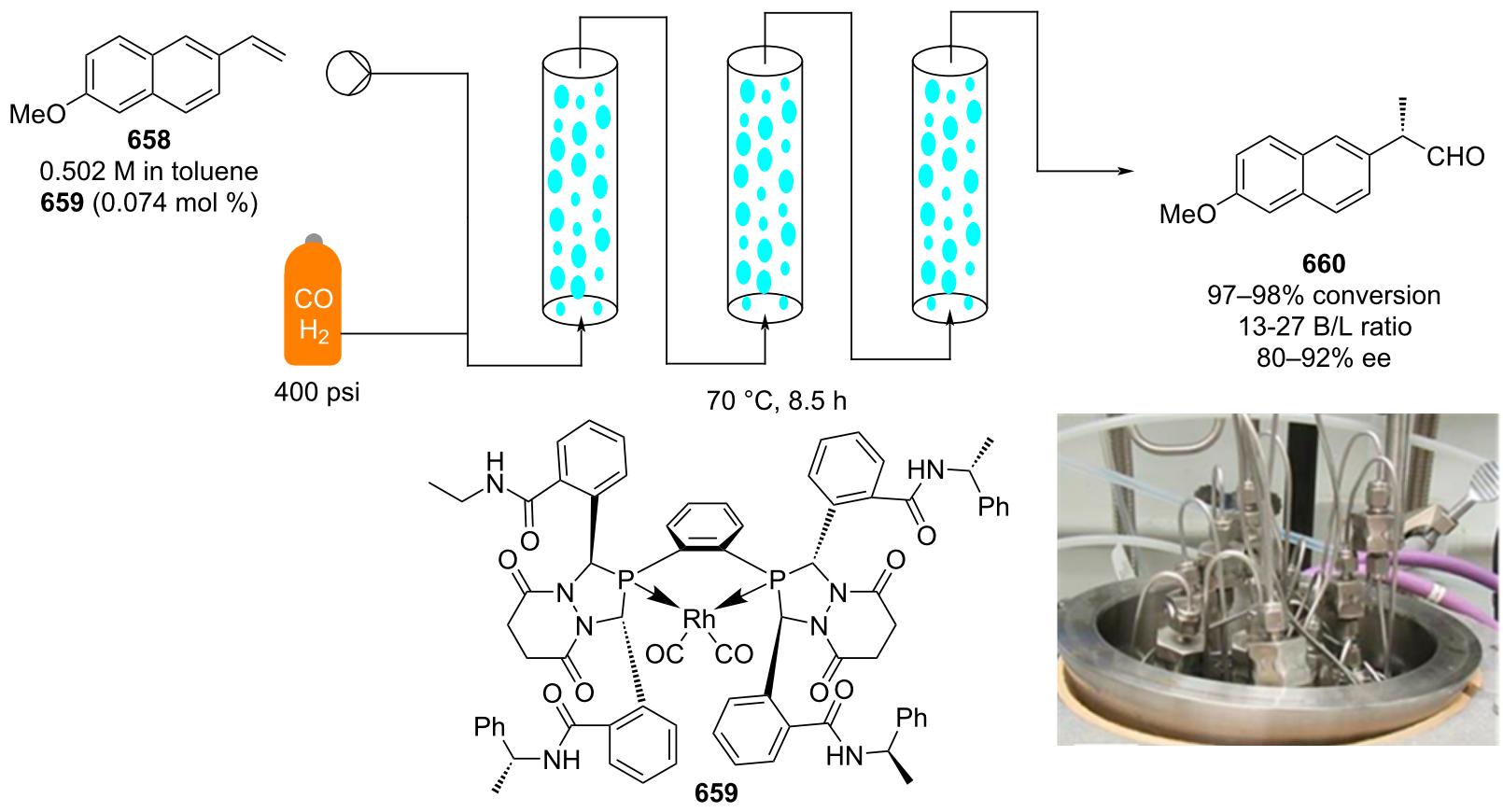

Scheme 153: Continuous-flow synthesis of the aldehyde 660 developed by Eli Lilly \& Co. [32]. Adapted with permission from [32]. Copyright 2016 American Chemical Society. 
immobilisation, the catalysts maintained their activity. The heterogeneous catalysts were then investigated in continuousflow mode using a U-shaped tube which was partially filled with cotton wool to enhance the initial gas-liquid mixing, and then partially filled with the catalyst bed (Scheme 154). The flow apparatus increases the enantioselectivity of the reaction $(661 \rightarrow$ 662), however, substantial metal leaching and activity loss was observed using MWCN, and rGO. Increasing the catalyst loading in the fixed bed and employing the CB-supported catalyst, the authors managed to setup a stable flow system allowing conversion up to $35 \%$ over 190 minutes of runtime with an ee of $70 \%$.

\section{Acetylation}

Acyl functionalities are often encountered in both fragrance products and their intermediates. Indeed, acetylation is a widely used transformation throughout of all synthetic chemistry; used as a protecting group, for activating an alcohol or as small molecular weight esters. As examples of acetylation in $F \& F$ industry, bornyl $(S, R, S$-664) and isobornyl $(S, S, S$-664) acetate are prepared via acetylation of their correspondent terpene derivatives such as borneol (665), pinene (S,S-314), and camphene $(S, R-610$, Scheme 155) [566].

These above processes are acid-catalysed reactions that go via carbocation intermediates. Therefore, the control of the conditions and the nature of the catalyst are essential to the resulting product composition. A continuous-flow approach for the synthesis of compound $S, R, S-\mathbf{6 6 4}$ has been reported using an oscillatory flow reactor and a heterogeneous catalyst (an acid cation exchange resin). The homogenous heat control and excellent dispersity of the catalyst in the reactor resulted in a higher
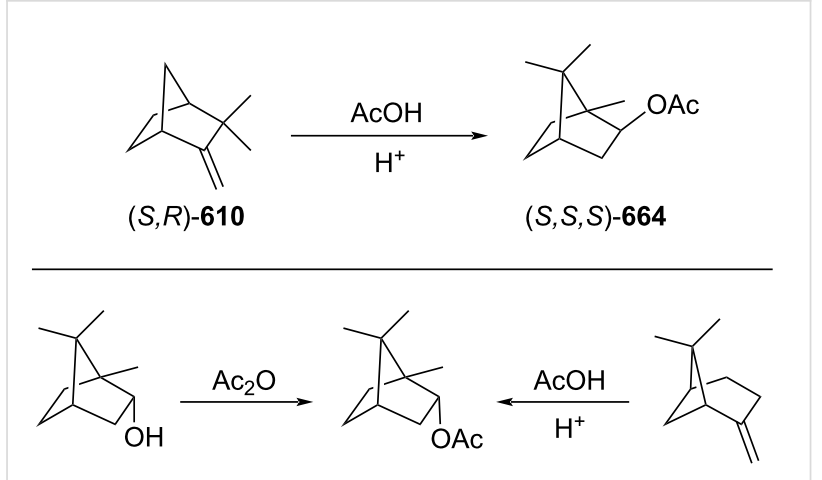

665

$(S, R, S)-664$

$(S, S)-314$

Scheme 155: Examples of acetylation in F\&F industry: synthesis of bornyl $(S, R, S-664)$ and isobornyl $(S, S, S-664)$ acetate.

selectivity and overall faster reaction compared to its corresponding batch mode (Scheme 156) [567].

Another example of F\&F chemical preparation is the acetylation of geraniol (343) through acetic anhydride developed by Adarme and co-workers in 2018 (Scheme 157) [568].

The reagent streams were combined together neat and passed through a heated coil reactor at $150{ }^{\circ} \mathrm{C}$. After 4 minutes, the reaction mixture was quenched in-line with a $10 \%$ solution of $\mathrm{Na}_{2} \mathrm{CO}_{3}$, thoroughly mixed using a glass beads-filled reactor, and then the two phases were continuously separated. The process lead to the final material $\mathbf{6 6 6}$ in $94.1 \%$ of purity $(99 \%$ conversion). The authors also developed a biocatalysed transesterification using ethyl acetate as the acetylating agent to yield the same material in high conversion ( $99 \%$ conversion).

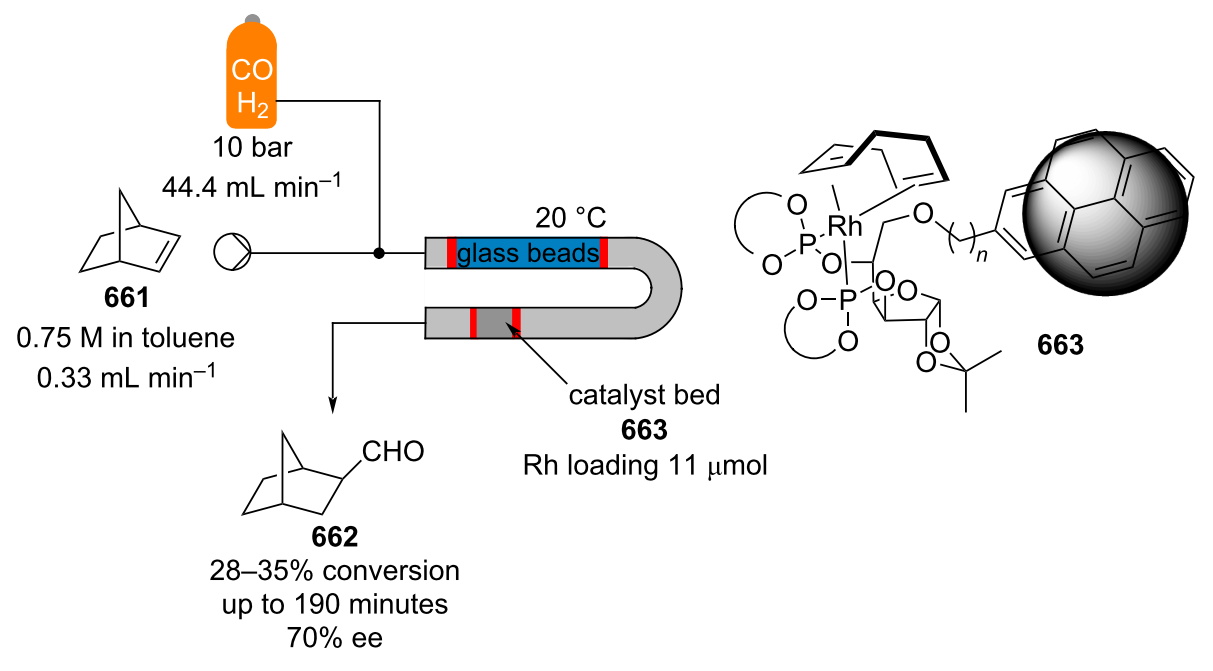

Scheme 154: Continuous asymmetric hydroformylation employing heterogenous catalst supported on carbon-based supports. 


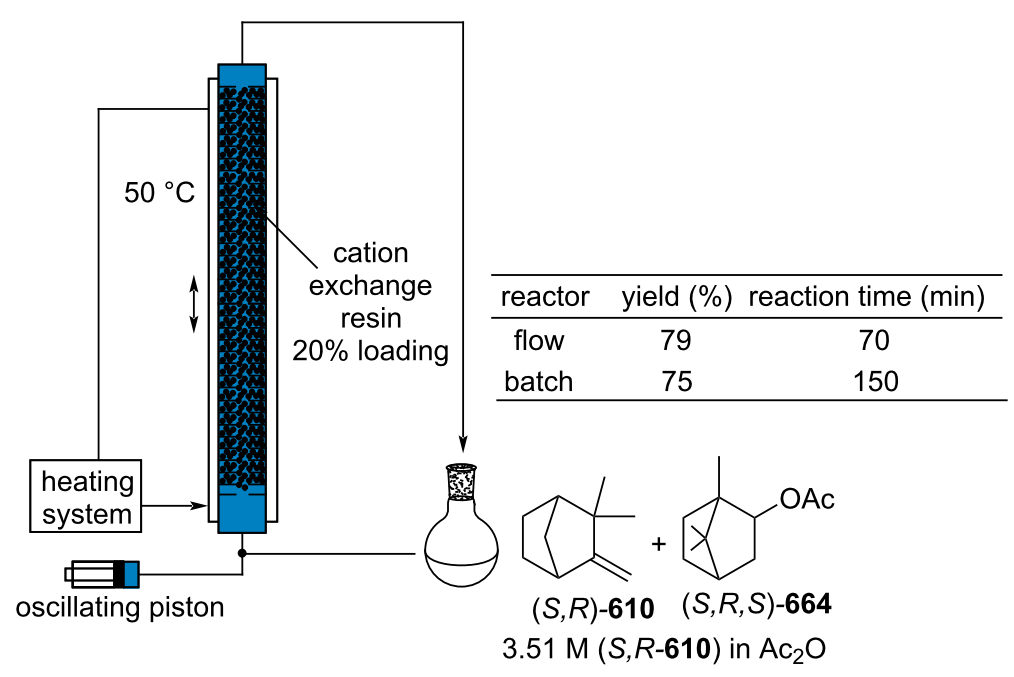

Scheme 156: Continuous-flow preparation of bornyl acetate $(S, R, S-664)$ employing the oscillating flow reactor.

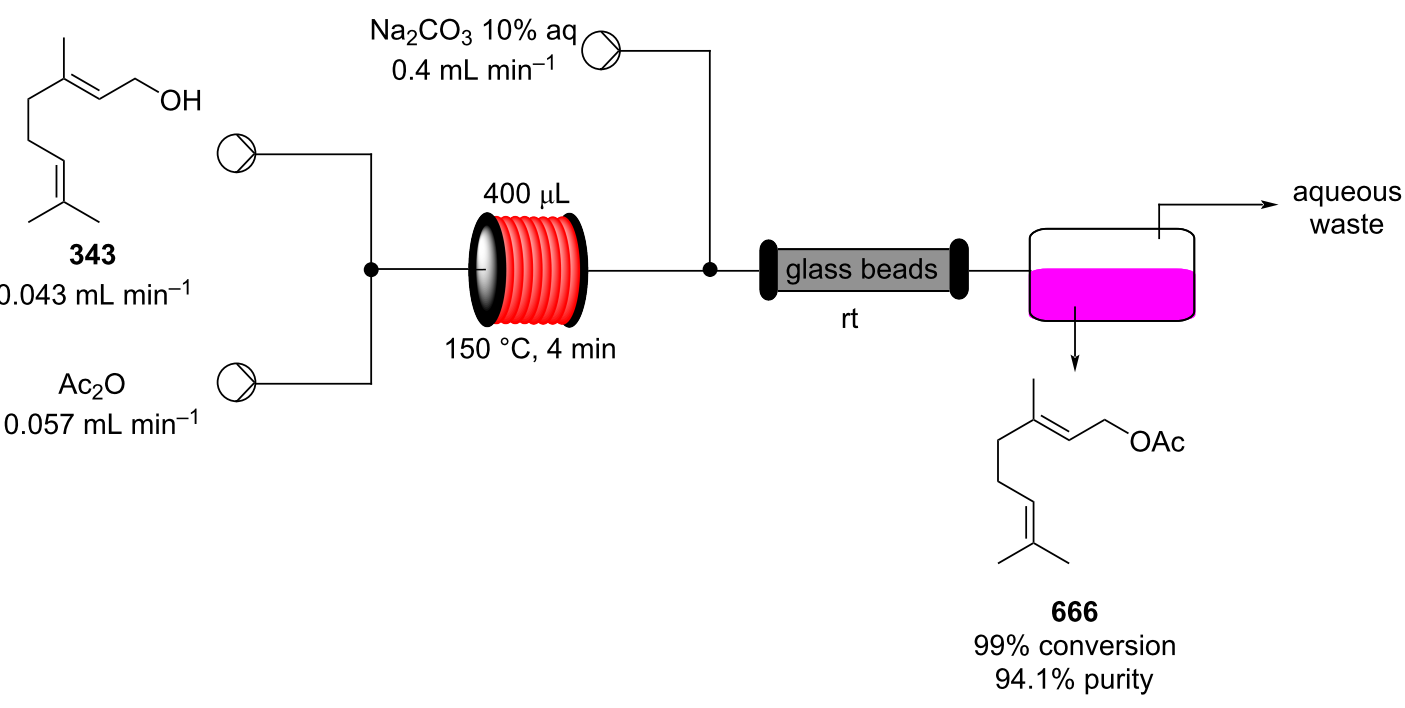

Scheme 157: Continuous-flow synthesis of geranyl acetate (666) from acetylation of geraniol (343) developed by Adarme et al.

Only a handful of reports exist that deal exclusively with acetylation in flow. In 2015 a polymer grafted dimethylaminopyridine ( $g$-DMAP) catalyst was used to promote a continuous acetylation as disclosed by Takeda and co-workers [569]. The immobilised reagent was developed as an alternative to DMAP supported on polystyrene ( $2 \%$ cross-linked with divinylbenzene) and was shown to give superior yields both in batch and flow. The starting material 1-phenyl-1-hexanol (427) was used as a model substrate, for which, it was found that the use of a $1.5 \mathrm{~mm}$ i.d. packed-bed reactor gave the best results. The packed column reactor was found to be highly robust, giving consistent conversions over the course of 50 hours. The reactors use in parallel for scale-up was also investigated
(Table 14). Good scalability was demonstrated with the authors using up to 8 parallel reactors simultaneously.

In 2012 the use of a 12-tungstosilicic acid-supported silica monolith $\left(\mathrm{H}_{4} \mathrm{SiW}_{12} \mathrm{O}_{40}\right.$-monolith) for the acetylation of a range of substrates in flow was reported by Haswell et al. (Scheme 158) [570].

The reactors were prepared in two sizes by immobilising the heteropolyacid (HPA) onto the monolith by adsorption and calcination. They were then clad in a heat-shrinkable Teflon ${ }^{\circledR}$ tube equipped with glass connectors at each end and heated to $330{ }^{\circ} \mathrm{C}$. The resultant columns were used for the acetylation of 
Table 14: Conversion values for individual reactors when used in parallel for continuous g-DMAP catalysed acetylation scale-up.

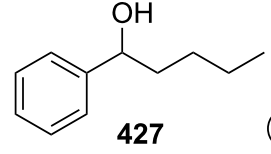

$0.3 \mathrm{M}$ in toluene $E t_{3} N(1.88$ equiv) $50 \mu \mathrm{L} \min ^{-1}$ reactor $^{-1}$

$\mathrm{Ac}_{2} \mathrm{O}$ (1.5 equiv)

$50 \mu \mathrm{L} \mathrm{min}^{-1}$ reactor

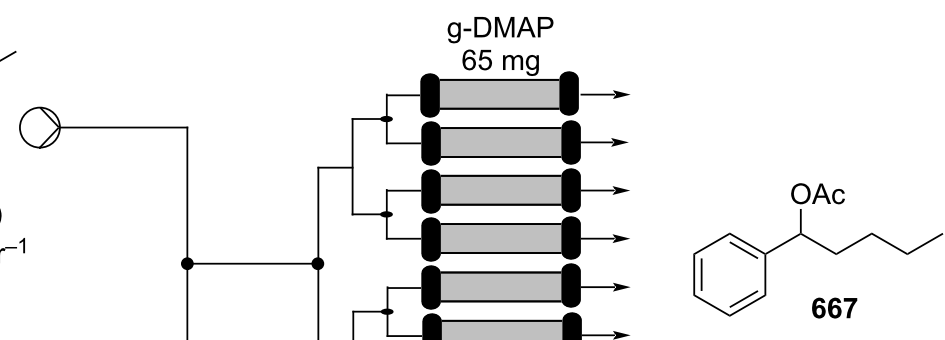

667

\begin{tabular}{|c|c|c|c|c|c|c|c|c|c|}
\hline \multirow[t]{2}{*}{ Entry } & \multirow[t]{2}{*}{ Number of parallel reactors } & \multicolumn{8}{|c|}{$\begin{array}{l}\text { Conversion }(\%)^{a} \\
\text { reactor number }\end{array}$} \\
\hline & & 1 & 2 & 3 & 4 & 5 & 6 & 7 & 8 \\
\hline 1 & 2 & 99 & 98 & - & - & - & - & - & - \\
\hline 2 & 3 & 94 & 99 & 97 & 95 & - & - & - & - \\
\hline 3 & 4 & 98 & 98 & 102 & 102 & 97 & 100 & 98 & 99 \\
\hline
\end{tabular}

aDetermined by HPLC.<smiles>O=[N+]([O-])C1CCC(O)CC1</smiles>

668

$0.3 \mathrm{M}$ in $\mathrm{Ac}_{2} \mathrm{O}$

$0.32 \mathrm{~mL} \mathrm{~min}^{-1}$

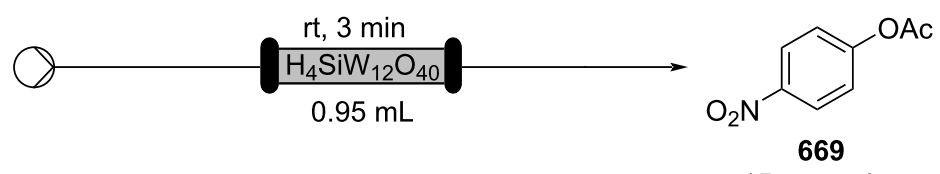

15 examples

97-100\%

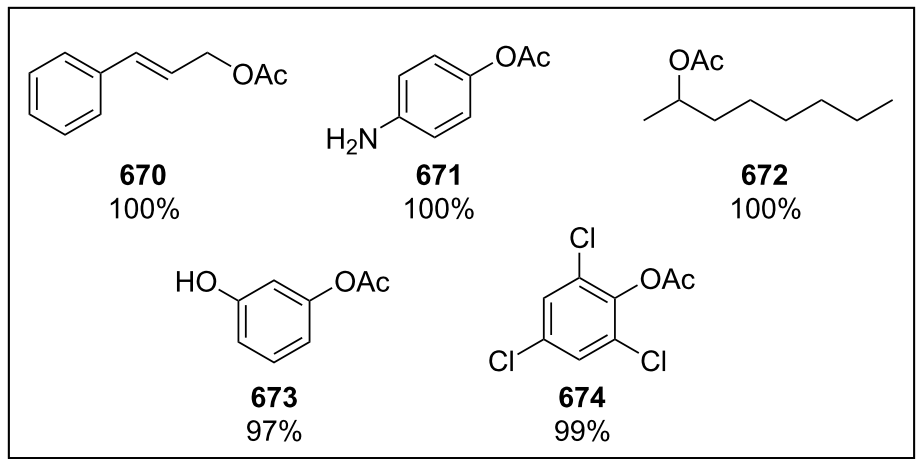

Scheme 158: 12-Ttungstosilicic acid-supported silica monolith-catalysed acetylation in flow.

various aromatic and aliphatic alcohols at room temperature with very high yields being obtained in all cases.

Another example of solid-supported catalyst is the acetylation of D-glucono-1,5-lactone (675) for the telescoped preparation of cyclopentenone 676 [571]. A CSTR system was developed where acetic anhydride and $\mathbf{6 7 5}$ were slowly added to a silicasupported sulfuric acid catalyst suspended in an stirred flask.
The calculated residence time was around 20 minutes and the penta-acetylated material was obtained in quantitative yield with a productivity of roughly $200 \mathrm{~g} \mathrm{~h}^{-1}$. When evaluated in batch mode, the authors noticed the reaction requires high amounts of $\mathrm{Ac}_{2} \mathrm{O}$ (4.5 equiv) and a precise control of the temperature to ensure high and clean conversion. Due to a low solublity of the lactone $\mathbf{6 7 5}$ in the acetylating agent, the continuous apparatus could only employ a CSTR, where the starting 
material solubilises during reaction. In the CSTR only 3.6 equivalents of $\mathrm{Ac}_{2} \mathrm{O}$ were employed enabling telescoping into a subsequent transformation. As such, in Scheme 159, a base-catalysed elimination followed by a methanolysis reaction were sequenced.

The final product 676 was then purified with a countercurrent extraction, employing a AM technologies ACR device allowing isolation of 676 in $48-50 \%$ overall yield. The authors also described a more efficient alternative procedure which employed an ion exchange resin for the last step. In this case, the cyclopentenone 676 was isolated in $71 \%$ yield with a producvtivity of $112 \mathrm{~g}$ over a $30 \mathrm{~h}$ runtime.

The acetylation of salicylaldehyde (88) in a continuous twostage synthesis of coumarin (90) has also described [572]. The first step was performed mixing a solution of $\mathbf{8 8}$ with a stream containing acetic anhydride and potassium acetate in acetic acid as the solvent. These were directed into a heated coil reactor maintained at $150{ }^{\circ} \mathrm{C}$. Complete conversion to the acetylated compound was reported, which converts to coumarin (90) after heating at $240{ }^{\circ} \mathrm{C}$. This process resulted in much shorter reaction times compared to working in batch and the apparatus was used to scale-up and allow collection of $120 \mathrm{~g}$ of material (Scheme 160).

Other examples of acetylation in flow include the acylation of racemic alcohols by lipases in continuous-flow bioreactors [573] and the high temperature transesterification of glycerol derivatives with alkyl and vinyl acetates [574]. In 2019, intensification process for the synthesis of melatonin was achieved employing solid-supported enzymes (Scheme 161) [575].

The reaction consists of a transesterification using ethyl acetate by MsAcT, which is an acetotransferase known for its remarkable activity. The authors investigated several hydrophilic

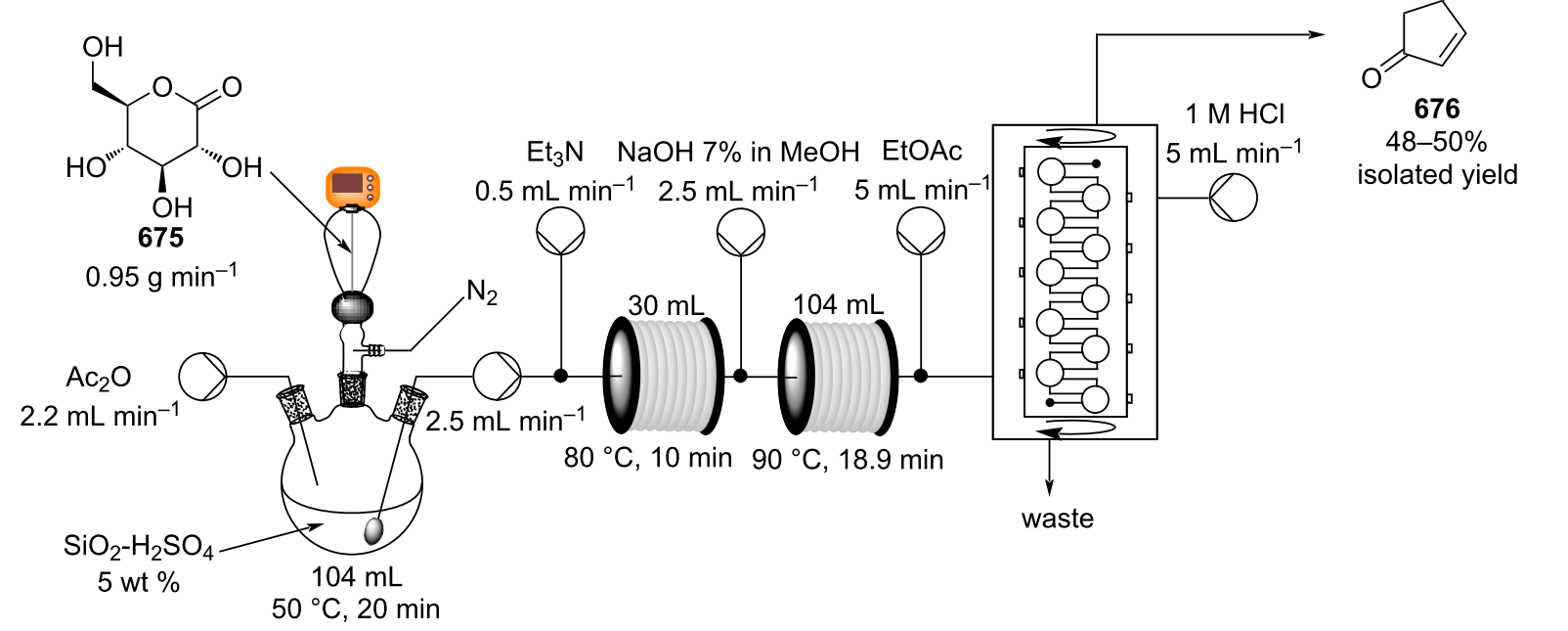

Scheme 159: Continuous-flow preparation of cyclopentenone 676<smiles>O=Cc1ccccc1O</smiles>

88

$0.22 \mathrm{~mL} \mathrm{~min}^{-1}$

$\mathrm{Ac}_{2} \mathrm{O}$

$\operatorname{AcOK}(0.01$ equiv)

$9.21 \mathrm{M}$ in $\mathrm{AcOH}$

$0.22 \mathrm{~mL} \mathrm{~min}^{-1}$

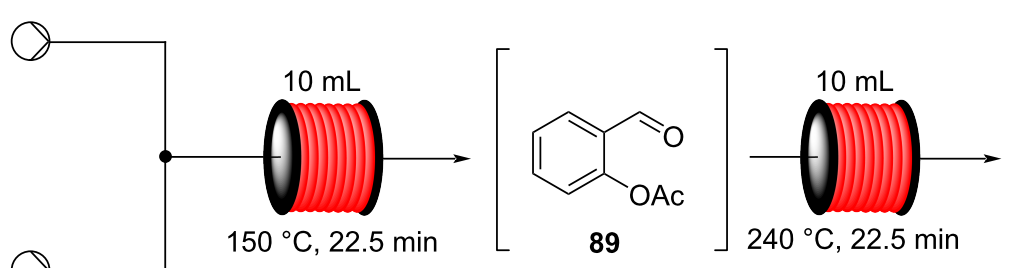

$91 \%$ conversion scaled-up to $120 \mathrm{~g}$ 


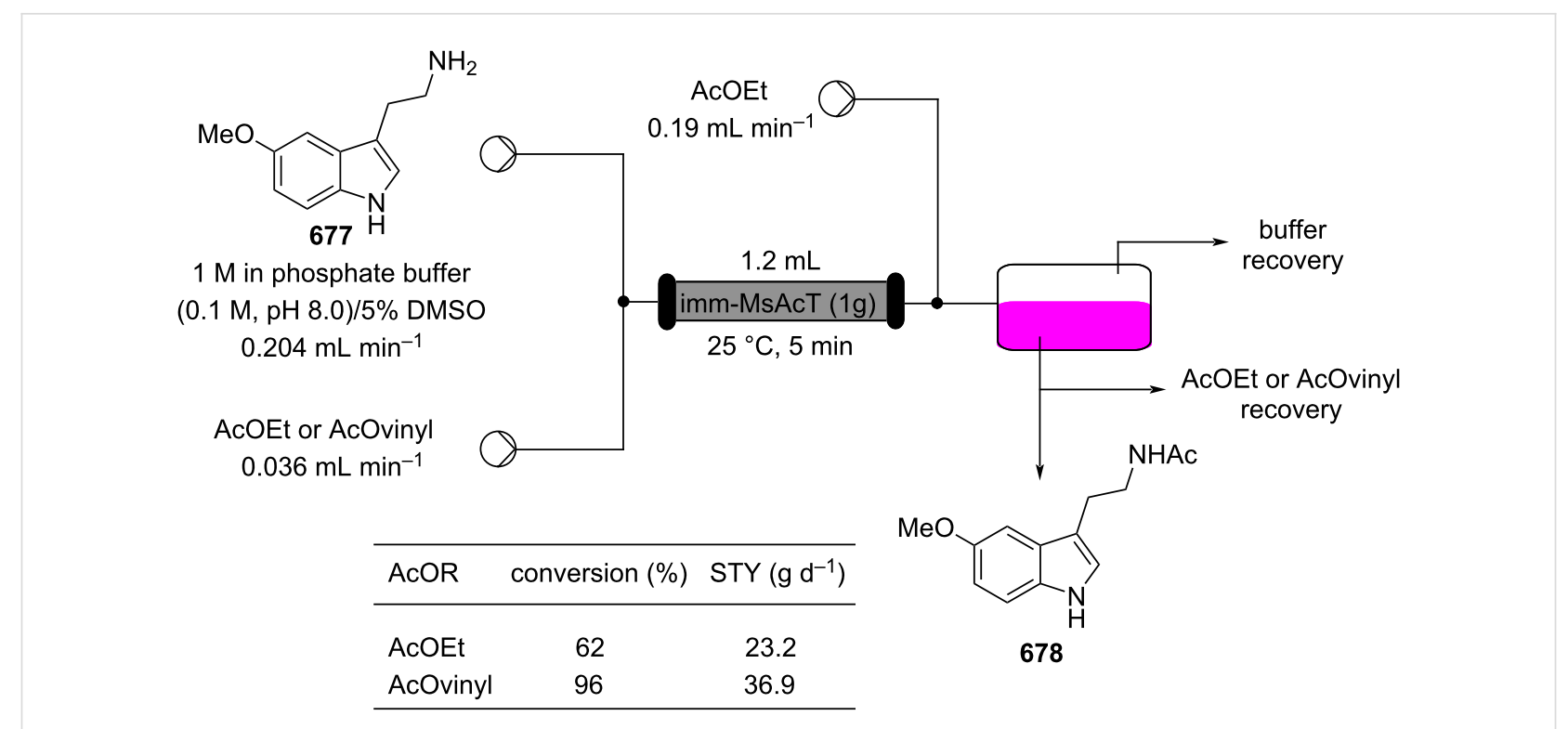

Scheme 161: Intensification process for acetylation of 5-methoxytryptamine (677) to melatonin (678) developed by Paradisi, Tamborini, and co-workers.

carriers and found out that glyoxyl agarose was the most suitable. Once packed in a column reactor, a $1 \mathrm{M}$ solution of tryptamine 677 in 85:15 pH 8 buffer/AcOEt was streamed though at $25{ }^{\circ} \mathrm{C}$. The heterogeneous mixture was then separated using a Zaiput liquid/liquid separator to yield melatonin in $62 \%$ conversions. Higher isolated yields (96\%) were obtained when vinyl acetate was used as an acetyl donor. The flow system allowed the authors to prepare roughly $36.9 \mathrm{~g} \mathrm{~d}^{-1}$ of the desired material 678. While there are numerous reports that feature acetylation as part of a multistep flow sequence [67,125,426,576], there is still much work to be done.

\section{Metathesis}

Discovered in the early 1950 s, olefin metathesis (OM) is a ground-breaking tool for organic synthesis, as highlighted by the award of the 2005 Nobel prize in chemistry jointly to Yves Chauvin, Robert H. Grubbs and Richard R. Schrock "for the development of the metathesis method in organic synthesis". Since the development and commercialisation of the first generation Grubbs catalyst, OM has found many applications [577] and it has now been industrially applied for the preparation of petrochemicals [578], polymers [579], and drugs [580,581]. Often disregarded by the F\&F industry due to issues with patent coverage and freedom to operate, there is now with many of the original patents expiring, a renewed interest, particularly for large ring formations. Ring-closing metathesis (RCM) allows for the easily formation of medium-large size rings, which would be less practicable using alternative strategies. Several catalysts have been developed for this task, achieving high activities (with TONs up to 315,000 ) and selectivities [582-585].
Beyond a few exceptional examples on small rings (damascone and galbanone derivatives) [586,587], the RCM has found more application in the synthesis of macrocyclic musky odorants. Starting from the natural civettone (679), muscone (680), and omega-pentadecalactone (681), numerous medium size macrocycles have been developed and are now commercially available (Scheme 162).

At the end of the twentieth century, only a few reported syntheses employing OM were known [588-590], often due to issues with selectivity and poor activity of the catalysts. Later, with progress in the catalyst design (especially stabilising ligand framework), various multistep preparations of macrocycles were being described [591-599]. However, common to all these examples is that ring-closing metathesis requires high dilution to avoid dimerization and as such it also needs long reaction times, a problematic issue when considering industrially application. Consequently, enabling technologies such as flow chemistry have been employed to aid with process intensification.

In 2005, Organ and Comer employed a flow OM setup combined with microwave irradiation to boost reaction times from 30 minutes to as little as 3 minutes yielding the products in full conversion (Scheme 163) [600]. Similar improvements were obtained when RCM was employed for the formation of smaller rings [601].

Lamaty et al. described a continuous-flow setup where a reaction in dimethyl carbonate (DMC) was passed into the coil at $110-120{ }^{\circ} \mathrm{C}$ for $1-5$ minutes and 2,5-dihydro- $1 H$-pyrroles were 


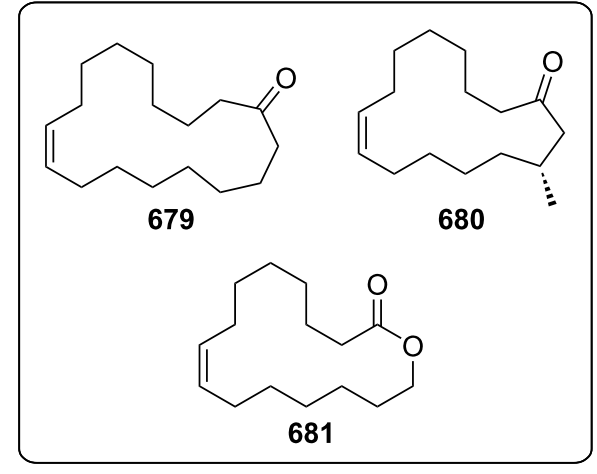

natural<smiles>O=C1CCCCCC/C=C\C/C=C/CCOC1</smiles>

682<smiles>CCCCCCCCCC(=O)OCCOC(=O)CCCCCCCCC</smiles>

synthetic

Scheme 162: Examples of macrocyclic musky odorants both natural (679-681) and synthetic (682 and 683).<smiles>C=CC(NCCCCCCCCCC)C(=O)N(CC(=O)OC(C)(C)C)c1ccccc1</smiles>

684

$685(1 \mathrm{~mol} \%)$

$0.15 \mathrm{M}$ in DCM
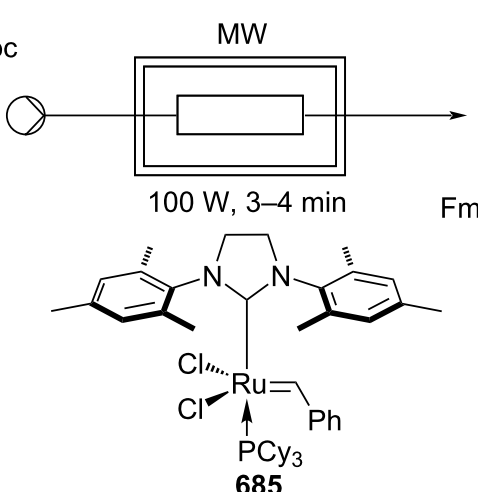

Fmoc-NH O

686

$100 \%$ conversion

Scheme 163: Flow setup combined with microwave for the synthesis of macrocycle 686 via RCM.

isolated in moderate to high yields (Scheme 164) [602]. The system allowed the larger scale processing of $\mathbf{6 8 7}(10 \mathrm{~g})$ and after 37 minutes the product $\mathbf{6 8 9}$ was isolated in $91 \%$ yield, with comparable result as obtained on a $1 \mathrm{mmol}$ scale in batch.
Leadbeater et al. applied a similar system to several other feedstocks such as linalool, citronellene (485), and eugenol which gave moderate to high conversions $(40-100 \%)$ to their products 691-693 in residence times of 10-30 minutes (Scheme 165) [603].<smiles>C=CCN([Te])C(C(=C)C(C)=O)c1ccccc1[N+](=O)[O-]</smiles>

687

$688(3 \mathrm{~mol} \%)$ $0.25 \mathrm{M}$ in DMC $4 \mathrm{~mL} \mathrm{~min}{ }^{-1}$
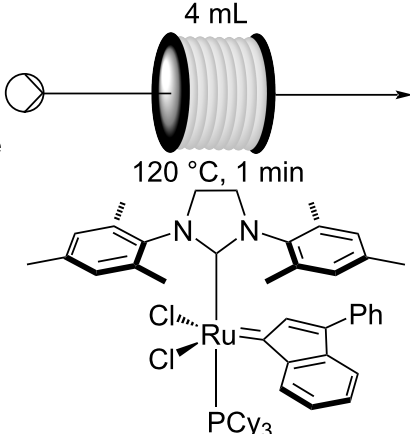

688<smiles>COC(=O)C1=CCN([As])C1c1ccccc1[N+](=O)[O-]</smiles>

689

$91 \%$ isolated yield on 10 gram-scale

6 examples $52-92 \%$ Isolated yield 

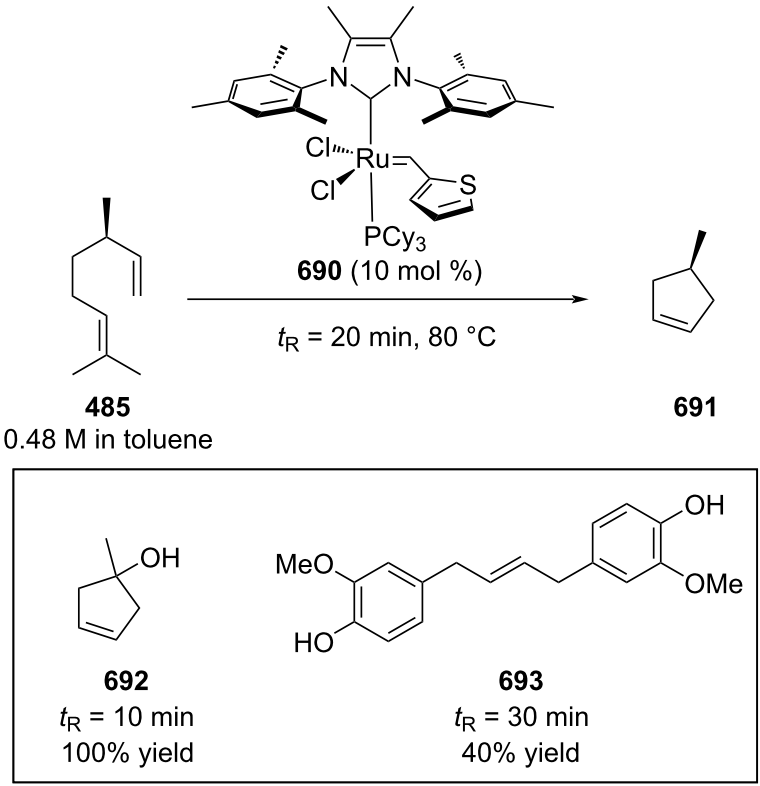

Scheme 165: Continuous-flow metathesis of $\mathbf{4 8 5}$ developed by Leadbeater et al.
Recently, ring-closing metathesis has been used in the synthesis of 3-methylcyclohexandec-6-enone (696), a Firmenich musky odorant product, which was performed in a continuousflow mode (Figure 12). The results were then compared to the reaction obtained in batch under conventional thermal and with microwave heating [604].

Although showing lower yields ( $32 \%$ vs $57 \%$ ), the flow and microwave reactions allowed the reaction to be conducted at a higher concentration (10 $\mathrm{mM}$ vs $5 \mathrm{mM}$ ) and gave much shorter reaction times (5-60 minutes vs 5 days). Additionally, the flow protocol was easily scaled up, yielding $1 \mathrm{~g}$ of product 696 in less than 5 hours. The high percentage of unreacted starting material suggests a more selective reaction and presents the possibility to recover unreacted starting material. The methodology was also further exploited in the synthesis of different 16-membered analogues and resulted in highly reproducible processes.

A general issue in many catalysis processes is the need for careful final purification of the material to remove residual metal and ligands, which is not always trivial. This is especially true as many examples still required high loading of the
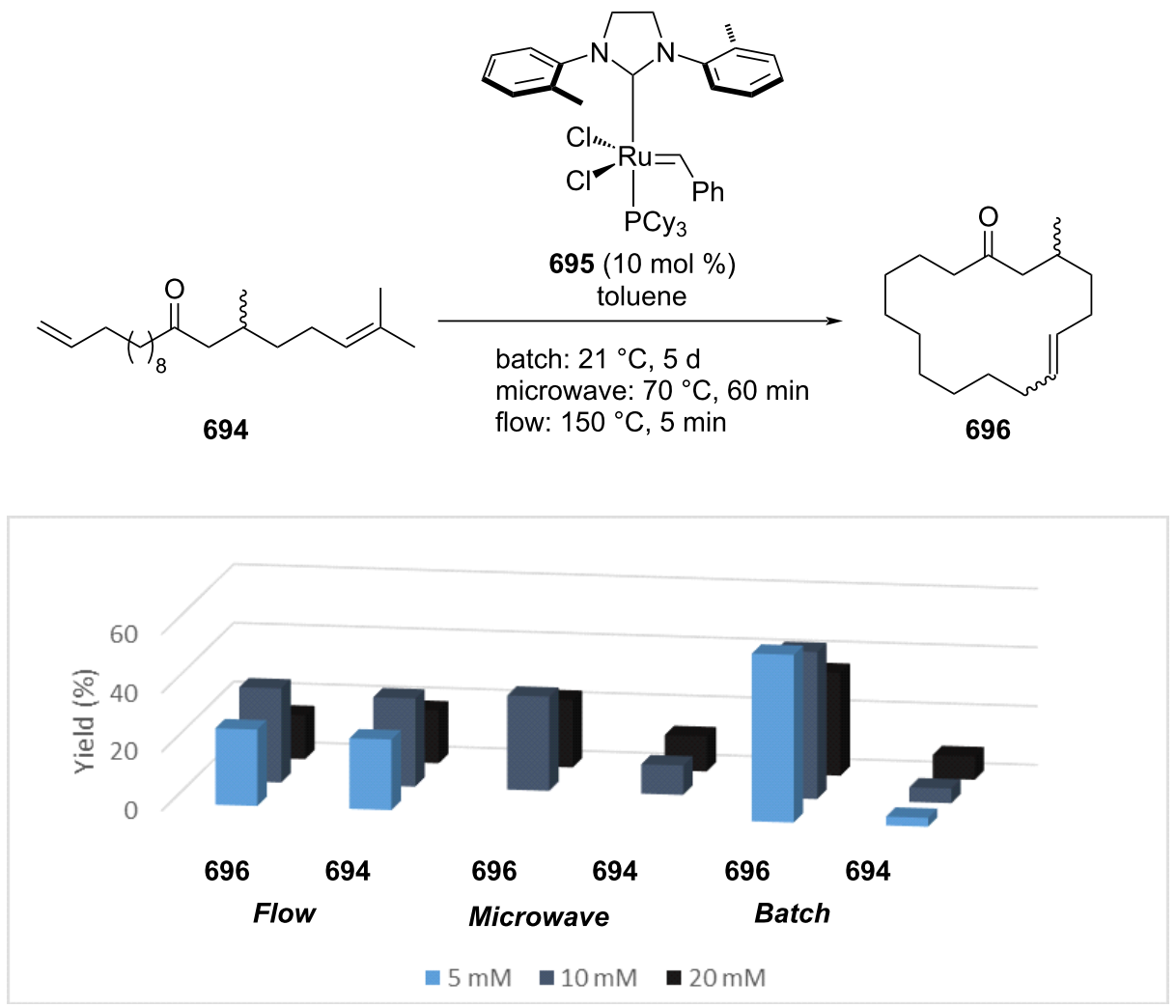

Figure 12: Comparison between RCM performed using different routes for the preparation of 696 . On the left the conversion of 696 and the percentage of unreacted material 694 on the right. 
catalyst and this also raises the issue of its necessary recovery due to the high metal costs. One possible option is to move to heterogeneous catalysis, but this still presents issues in terms of activity and recyclability.

As an excellent example of heterogeneous catalysis, Kirschning, Grela, and co-workers performed ruthenium immobilisation on a sulfonic acid resin by means of ionic interactions [605]. An amine-functionalised ligand was flushed through a sulfonic acid functionalised composite material shaped to fit in a stainless steel column (Scheme 166).

The ruthenium catalyst 698 was then installed via cross-metathesis of Grubbs II catalyst with the solid support. Despite the advantages in employing such immobilisation (fast support recovery and catalyst regeneration on large scales), the authors described a rapid drop in catalytic activity after 2 runs (100\% first run, $79 \%$ second run, $0 \%$ third run) when applied to the RCM of 697.

In 2012, Limbach et al. described a continuous-flow system equipped with a catalyst absorbed on silica gel [606]. The catalyst presents amino groups on the $N$-heterocyclic carbene moieties which enables ionic interactions between the catalyst and the silanol groups of the silica gel. The most active catalyst 700 (TON $=4350$ ) was used for ring-opening/ring-closing metathesis (RORCM) of cyclooctene (257), however, it achieved low selectivity for the desired dimer $\mathbf{7 0 1}$ (only 19\%, Scheme 167). The system was also exploited for the self-metathesis of methyl oleate (703), where turnover numbers of 4950 was used, but unfortunately, coupled with moderate Rh leaching $(19 \%)$.

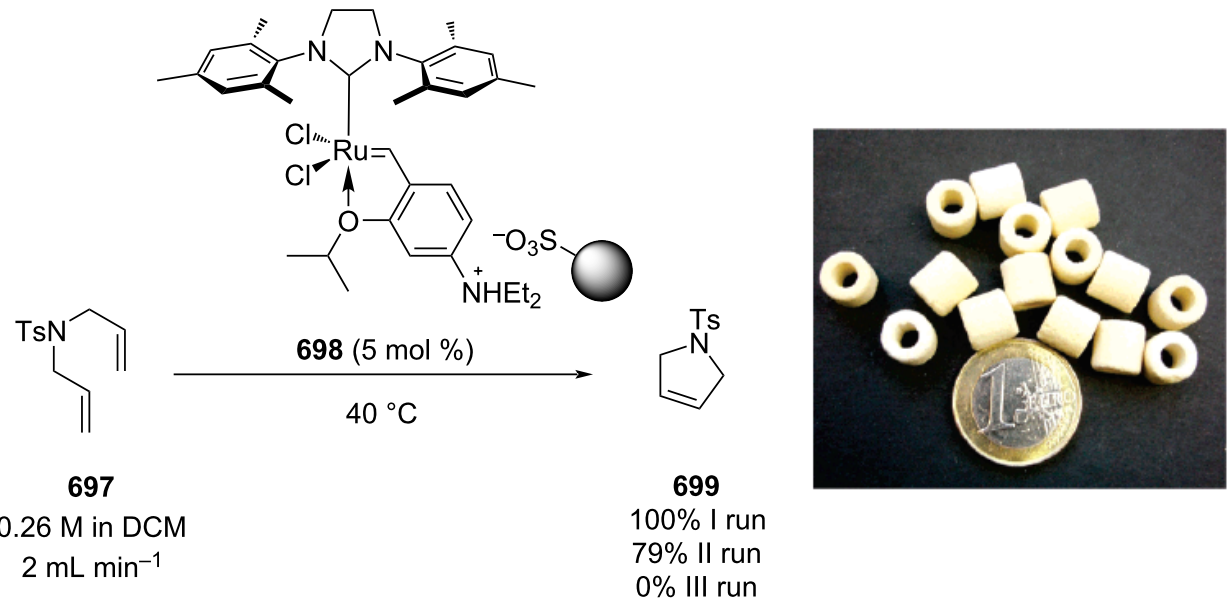

Scheme 166: Continuous-flow RCM of 697 employed the solid-supported catalyst 698 developed by Grela, Kirschning et al. [605] Picture showing the glass Raschig rings compared to a $1 €$ coin's dimension. Adapted with permission from [605]. Copyright 2006 American Chemical Society.

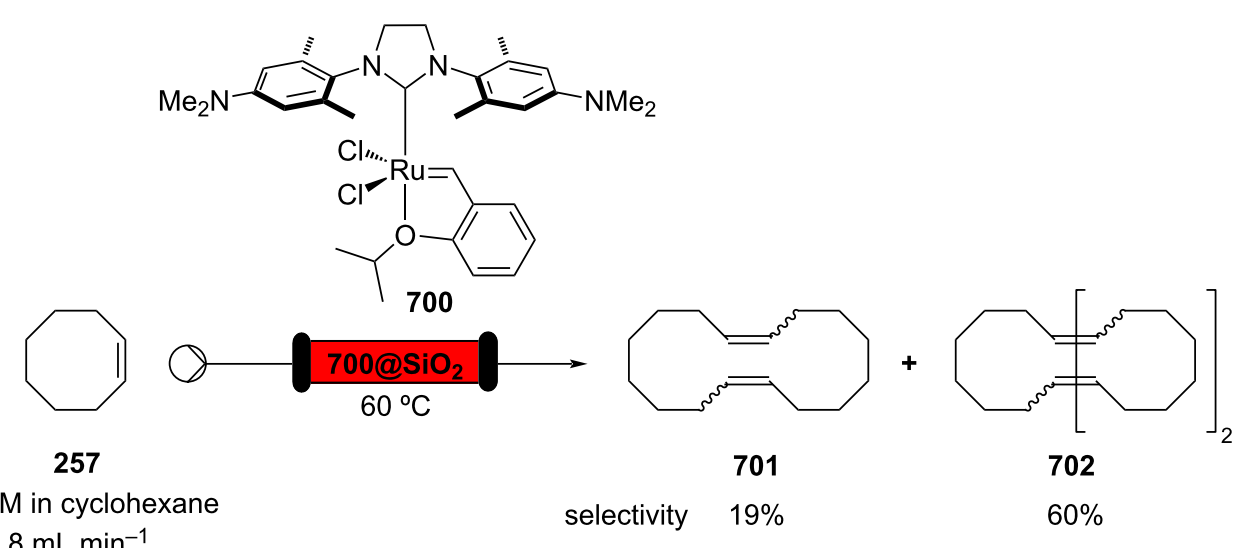

Scheme 167: Continuous-flow RORCM of cyclooctene employing the silica-absorbed catalyst 700 . 
Over the years, other immobilization concepts have been investigated. The immobilization strategy was performed through either impregnation-absorption [607] or binding of the metal to a ligand on the support [605,608-613]. Several supports were evaluated such as silica gel $[606,608,613]$, mesoporous silica [607,609,610], polymers [612], and oligomers [611]. A rhenium catalyst $\left(\mathrm{Re}_{2} \mathrm{O}_{7}\right)$ has also been reported in flow for the crossmetathesis of 1-octene (650) using $\mathrm{scCO}_{2}$ as the solvent to allow a better mass transport (higher pressure) and catalyst recovery [614].

Nolan et al. also employed an elegant catalyst immobilisation strategy involving impregnation of the supported ionic liquid phase (SILP) catalyst on silica [615]. The resulting silica-supported catalyst $\mathbf{7 0 4}$ was place into a $9 \mathrm{~mL}$ column reactor and the substrate were flushed through the system using $\mathrm{scCO}_{2}$ as the solvent (Scheme 168).

The system was found highly efficient for the self-metathesis of methyl oleate (703), reaching catalyst turnover numbers $>10000$, with low metal leaching ( $8 \mathrm{ppm}$ ). Cross-metathesis between substrate $\mathbf{7 0 3}$ and dimethyl maleate was also found to work well, however, with lower activity and therefore longer reaction time required. The system was however found unsuitable for metathesis of terminal alkenes.

More recently an alternative approach using an organic solvent nanofiltration (OSN) membrane for the RCM reaction has been reported [616-618]. The system uses a homogeneous catalyst but simplifies the catalyst removal by nanofiltration making it more industrially applicable. As an illustration Jensen et al. used an organic solvent nanofiltration (OSN) membrane to facilitate recovery of catalyst $\mathbf{7 0 7}$ enabling easy recycling (Scheme 169) [619].

In the reaction a co-feed of catalyst $707(2 \mathrm{mg})$ and substrate (ratio 1:1300) enabled 50 hours of continuous operation with a constant output of $94 \%$ of the desired product 699. Moreover, the metal content in the final material was less than $1 \mathrm{ppm}$. The authors highlighted the apparatus could be further optimised to maximize the catalyst TON, which was measured to be 950 .

Unfortunately, standard commercially available catalysts such as Hoveyda-Grubbs and Umicore M series [618], would be of too low molecular weight to allow their separation by current molecular membranes. For these reasons many modified higher molecular weight catalysts have been prepared and applied in continuous systems for a better catalyst recovery $[616,617]$.

Despite the extensive work on catalyst design and activity improvements, low catalyst activity and poor selectivity for certain substrates is still a big issue in the wider adoption of this reaction within industrial settings. As claimed by Fogg et al., the ethane produced by the reaction could stay entrapped in the system, causing catalyst's decomposition. Using catalyst $\mathbf{6 8 5}$, they obtained slightly better results using a continuous stirredtank reactor (CSTR) than a tubular flow reactor, confirming their hypothesis (Scheme 170) [620]. This is interesting and may offer further usage of reactor systems like the tube-in-tube reactor setup for the removal of volatiles such as ethane in flow processes to improve activity or change product compositions.

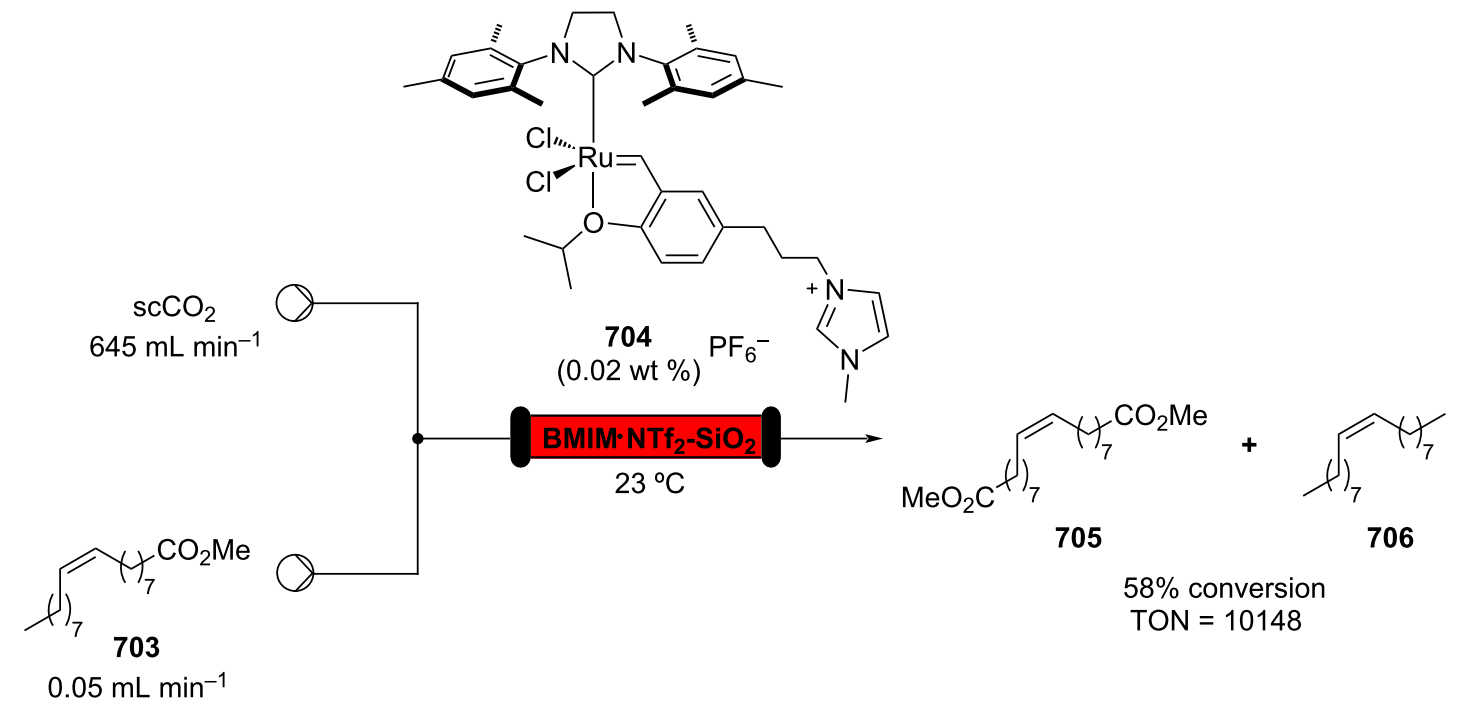

Scheme 168: Continuous-flow self-metathesis of methyl oleate (703) employing SILP catalyst 704. 


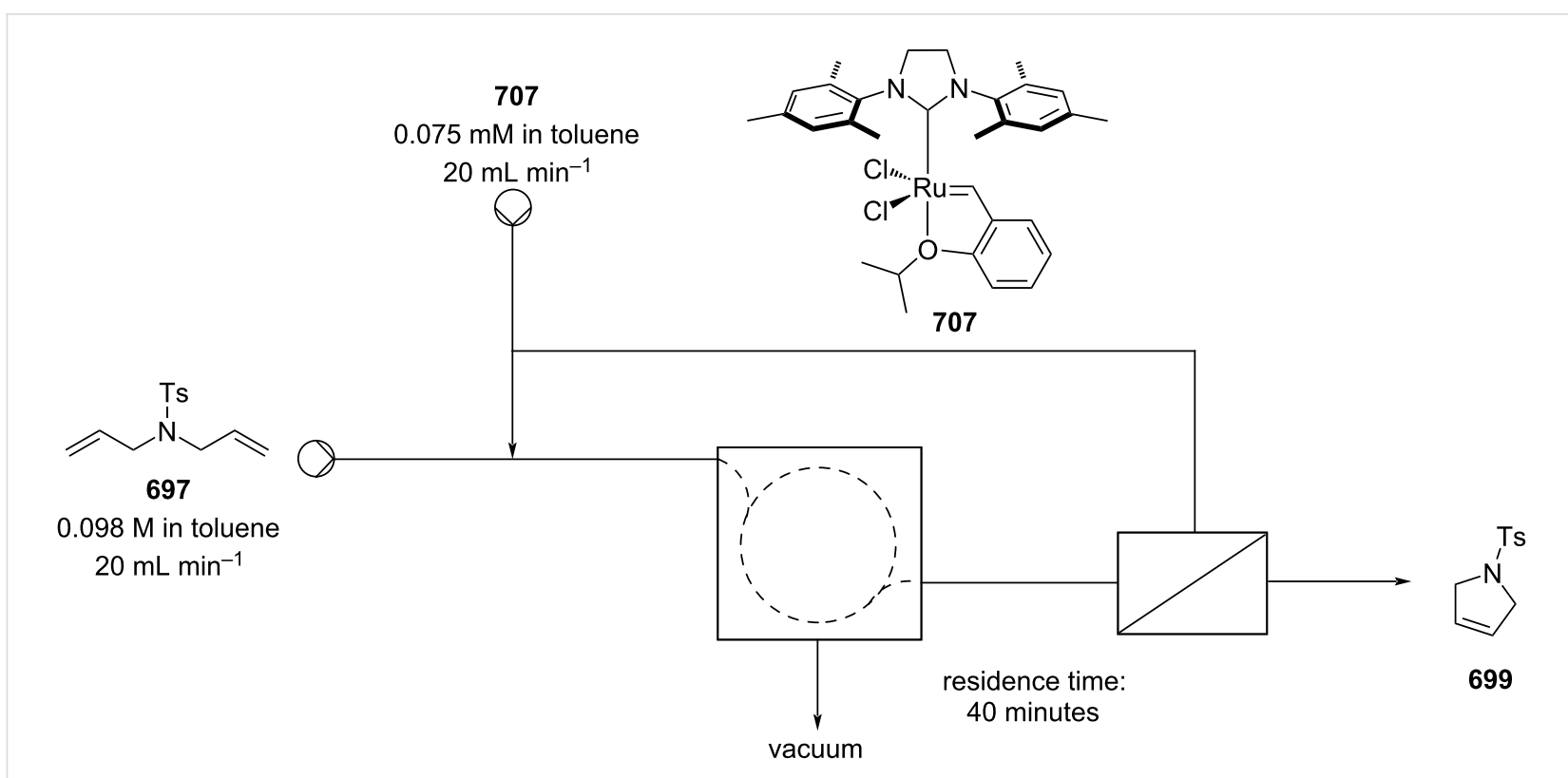

Scheme 169: Flow apparatus for the RCM of 697 using a nanofiltration membrane for the recovery and reuse of the catalyst 707.

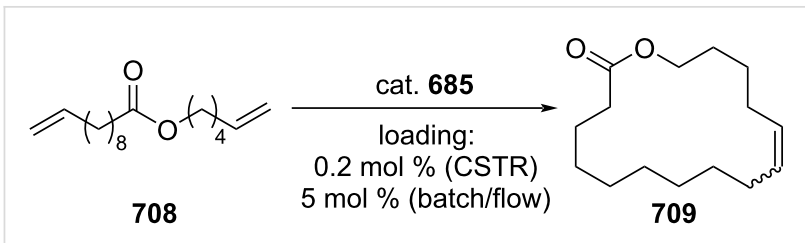

Scheme 170: Comparison of loadings between RCMs performed with different routes for the synthesis of 709 .

Indeed, this approach has been investigated by Jensen and O'Neal [619] and Skowerski et al. [621] who adopted a membrane reactor using Teflon AF2400 to allow the ethane removal by pervaporation (Scheme 169).

\section{Conclusion and Outlook}

Liquid fragrance ingredients and precursors are ideal candidates for continuous manufacturing. The majority of the examples in this review concerned smaller/research scale synthesis but in many cases scalability was also robust achieved. Other reaction types such as the Prins reaction and decarboxylation were not touched upon due to a lack of literature precedence. These should be acknowledged looking forward as should other problems associated with flow chemistry. There are currently limitations and weaknesses with regards to the integration of flow chemistry into manufacturing that need to be addressed. Much of the technology developed alongside flow equipment carries with it implications for various stages of production (camera integration [622], the use of feed-back algorithms [623] and in-line analytics techniques already mentioned), however, more research is needed to bring together flow chemistry and industrially relevant purification techniques such as distillation. Industry has generally been slow to adopt flow technology due to the "like what you know, know what you like" sentiment and the initial costs of investing in the necessary equipment to replace well established and well optimised processes. Despite this, the pharmaceutical industry is beginning to take advantage of the benefits offered by continuous manufacturing [624-626]. Undoubtedly, there are processes currently being used within the flavour and fragrance industry for which flow would offer an attractive alternative with regards to both cost and green/ environmental implications. These are very early days for flow within the fragrance industry, however, and much more work is needed to fully assess the feasibility of coupling the areas of flow chemistry and fragrance production.

\section{Funding}

We would like to thank International Flavors \& Fragrances Inc. (IFF) for funding (G.G. and J.S.S.).

\section{ORCID ${ }^{\circledR}$ iDs}

Guido Gambacorta - https://orcid.org/0000-0001-6603-7461

Ian R. Baxendale - https://orcid.org/0000-0003-1297-1552

\section{References}

1. Flavors and Fragrances Market Size, Share \& Industry Analysis, By Type (Flavors \{Natural and Synthetic\} and Fragrances \{Natural and Synthetic\}) By Application (Food \& Beverage, Cosmetics \& Personal Care, Pharmaceutical, Home \& Floor Care, Fine Fragrances. https://www.fortunebusinessinsights.com/flavors-and-fragrances-mark et-102329 (accessed Jan 4, 2021). 
2. Appendix I: Some of the More Important Natural Fragrance Materials. In The Chemistry of Fragrances: From Perfumer to Consumer, 2nd ed.; Sell, C. S., Ed.; The Royal Society of Chemistry: Cambridge. UK, 2006; p 348.

3. Fukuyama, T.; Rahman, M.; Sato, M.; Ryu, I. Synlett 2008, 151-163. doi:10.1055/s-2007-1000884

4. Baxendale, I. R.; Brocken, L.; Mallia, C. J. Green Process. Synth. 2013, 2, 211-230. doi:10.1515/gps-2013-0029

5. Ley, S. V.; Baxendale, I. R. Nat. Rev. Drug Discovery 2002, 1, 573-586. doi:10.1038/nrd871

6. Baxendale, I. R. J. Chem. Technol. Biotechnol. 2013, 88, 519-552. doi:10.1002/jctb.4012

7. Jas, G.; Kirschning, A. Chem. - Eur. J. 2003, 9, 5708-5723. doi: $10.1002 /$ chem. 200305212

8. Sell, C. S., Ed. The Chemistry of Fragrances: From Perfumer to Consumer, 2nd ed.; Royal Society of Chemistry: Cambridge. UK, 2006.

9. Bauer, K.; Garbe, D.; Surburg, H. Common Fragrance and Flavor Materials Preparation, Properties and Uses, 4th ed.; Wiley-VCH: Weinheim, Germany, 2001. doi:10.1002/3527600205

10. About the IFRA Transparency List. https://ifrafragrance.org/initiatives/transparency/ifra-transparency-list (accessed March 30, 2020).

11. Salvito, D.; Lapczynski, A.; Sachse-Vasquez, C.; Mclntosh, C.; Calow, P.; Greim, H.; Escher, B. Ecotoxicol. Environ. Saf. 2011, 74, 1619-1629. doi:10.1016/j.ecoenv.2011.05.002

12. Ohloff, G.; Pickenhagen, W.; Kraft, P. Scent and Chemistry. The Molecular World of Odors; Verlag Helvetica Chimica Acta and Wiley-VCH: Zurich, Switzerland, 2011.

13. Zarzo, M.; Stanton, D. T. Atten. Percept. Psychophys. 2009, 71 , 225-247. doi:10.3758/app.71.2.225

14. Webb, D.; Jamison, T. F. Chem. Sci. 2010, 1, 675-680. doi:10.1039/c0sc00381f

15. Wegner, J.; Ceylan, S.; Kirschning, A. Chem. Commun. 2011, 47, 4583-4592. doi:10.1039/c0cc05060a

16. Rossetti, I.; Compagnoni, M. Chem. Eng. J. 2016, 296, 56-70. doi:10.1016/j.cej.2016.02.119

17. Brzozowski, M.; O’Brien, M.; Ley, S. V.; Polyzos, A. Acc. Chem. Res. 2015, 48, 349-362. doi:10.1021/ar500359m

18. Ramanjaneyulu, B. T.; Vishwakarma, N. K.; Vidyacharan, S.; Adiyala, P. R.; Kim, D.-P. Bull. Korean Chem. Soc. 2018, 39, 757-772. doi:10.1002/bkcs.11467

19. Trojanowicz, M. Molecules 2020, 25, 1434. doi:10.3390/molecules25061434

20. Guidi, M.; Seeberger, P. H.; Gilmore, K. Chem. Soc. Rev. 2020, 49, 8910-8932. doi:10.1039/c9cs00832b

21. Britton, J.; Jamison, T. F. Nat. Protoc. 2017, 12, 2423-2446. doi:10.1038/nprot.2017.102

22. Darvas, F.; Dorman, G.; Hessel, V., Eds. Flow Chemistry Applications; Walter de Gruyter: Berlin, Germany, 2014. doi:10.1515/9783110367508

23. Vaccaro, L., Ed. Sustainable Flow Chemistry: Methods and Applications; Wiley-VCH: Weinheim, Germany, 2017. doi:10.1002/9783527689118

24. Jamison, T.; Koch, G., Eds. Flow Chemistry in Organic Synthesis; Science of Synthesis; Thieme: Stuttgart, Germany, 2018. doi:10.1055/b-00000101
25. Luis, S. V.; Garcia-Verdugo, E., Eds. Flow Chemistry: Integrated Approaches for Practical Applications; Green Chemistry Series, Vol. 62; Royal Society of Chemistry: Cambridge. UK, 2019. doi:10.1039/9781788016094

26. Len, C.; Luisi, R., Eds. Catalytic Methods in Flow Chemistry; MDPI: Switzerland, 2020.

27. Noël, T.; Luque, R., Eds. Accounts on Sustainable Flow Chemistry; Topics in Current Chemistry Collections; Springer International Publishing: Cham, Switzerland, 2020. doi:10.1007/978-3-030-36572-1

28. Kitching, M. O.; Dixon, O. E.; Baumann, M.; Baxendale, I. R. Eur. J. Org. Chem. 2017, 6540-6553. doi:10.1002/ejoc.201700904

29. Gérardy, R.; Emmanuel, N.; Toupy, T.; Kassin, V.-E.; Tshibalonza, N. N.; Schmitz, M.; Monbaliu, J.-C. M. Eur. J. Org. Chem. 2018, 2301-2351. doi:10.1002/ejoc.201800149

30. Baumann, M.; Baxendale, I. R. Beilstein J. Org. Chem. 2015, 11, 1194-1219. doi:10.3762/bjoc.11.134

31. Johnson, M. D.; May, S. A.; Calvin, J. R.; Lambertus, G. R.; Kokitkar, P. B.; Landis, C. R.; Jones, B. R.; Abrams, M. L.; Stout, J. R. Org. Process Res. Dev. 2016, 20, 888-900. doi:10.1021/acs.oprd.5b00407

32. Abrams, M. L.; Buser, J. Y.; Calvin, J. R.; Johnson, M. D.; Jones, B. R.; Lambertus, G.; Landis, C. R.; Martinelli, J. R.; May, S. A.; McFarland, A. D.; Stout, J. R. Org. Process Res. Dev. 2016, 20, 901-910. doi:10.1021/acs.oprd.5b00406

33. Kappe, C. O. Curr. Opin. Chem. Biol. 2002, 6, 314-320. doi:10.1016/s1367-5931(02)00306-x

34. Watts, P. QSAR Comb. Sci. 2005, 24, 701-711. doi:10.1002/qsar.200540004

35. Wiles, C.; Watts, P.; Haswell, S. J. Lab Chip 2007, 7, 322-330. doi:10.1039/b615069a

36. Pastre, J. C.; Browne, D. L.; Ley, S. V. Chem. Soc. Rev. 2013, 42 , 8849-8869. doi:10.1039/c3cs60246j

37. Baxendale, I.; Ley, S. Curr. Org. Chem. 2005, 9, 1521-1534. doi:10.2174/138527205774370513

38. Akwi, F. M.; Watts, P. Chem. Commun. 2018, 54, 13894-13928. doi:10.1039/c8cc07427e

39. Baumann, M.; Moody, T. S.; Smyth, M.; Wharry, S. Org. Process Res. Dev. 2020, 24, 1802-1813. doi:10.1021/acs.oprd.9b00524

40. Anastas, P.; Warner, J. Green Chemistry: Theory and Practice; Oxford University Press: Oxford, UK, 1998.

41. Wiles, C.; Watts, P. Green Chem. 2012, 14, 38-54. doi:10.1039/c1gc16022b

42. Anastas, P. T.; Zimmerman, J. B. Environ. Sci. Technol. 2003, 37, 94A-101A. doi:10.1021/es032373g

43. Sheldon, R. A. Green Chem. 2007, 9, 1273. doi:10.1039/b713736m

44. Browne, D. L.; Deadman, B. J.; Ashe, R.; Baxendale, I. R.; Ley, S. V. Org. Process Res. Dev. 2011, 15, 693-697. doi:10.1021/op2000223

45. Deadman, B. J.; Browne, D. L.; Baxendale, I. R.; Ley, S. V. Chem. Eng. Technol. 2015, 38, 259-264. doi:10.1002/ceat.201400445

46. Sharma, M. K.; Suru, A.; Joshi, A.; Kulkarni, A. A. Ind. Eng. Chem. Res. 2020, 59, 16462-16472. doi:10.1021/acs.iecr.9b06864

47. Bianchi, P.; Williams, J. D.; Kappe, C. O. J. Flow Chem. 2020, 10, 475-490. doi:10.1007/s41981-020-00105-6

48. Doyle, B. J.; Gutmann, B.; Bittel, M.; Hubler, T.; Macchi, A.; Roberge, D. M. Ind. Eng. Chem. Res. 2020, 59, 4007-4019. doi:10.1021/acs.iecr.9b04496 
49. Ley, S. V.; Baxendale, I. R.; Bream, R. N.; Jackson, P. S.; Leach, A. G.; Longbottom, D. A.; Nesi, M.; Scott, J. S.; Storer, R. I.; Taylor, S. J. J. Chem. Soc., Perkin Trans. 1 2000, 3815-4195. doi:10.1039/b006588i

50. Sussman, S. Ind. Eng. Chem., Ind. Ed. 1946, 38, 1228-1230. doi:10.1021/ie50444a010

51. Booth, R. J.; Hodges, J. C. Acc. Chem. Res. 1999, 32, 18-26. doi:10.1021/ar970311n

52. Adamo, A.; Heider, P. L.; Weeranoppanant, N.; Jensen, K. F. Ind. Eng. Chem. Res. 2013, 52, 10802-10808. doi:10.1021/ie401180t

53. Kralj, J. G.; Sahoo, H. R.; Jensen, K. F. Lab Chip 2007, 7, 256-263. doi:10.1039/b610888a

54. Sahoo, H. R.; Kralj, J. G.; Jensen, K. F. Angew. Chem. 2007, 119, 5806-5810. doi:10.1002/ange.200701434

55. Hornung, C. H.; Mackley, M. R.; Baxendale, I. R.; Ley, S. V. Org. Process Res. Dev. 2007, 11, 399-405. doi:10.1021/op700015f

56. Mason, B. P.; Price, K. E.; Steinbacher, J. L.; Bogdan, A. R.; McQuade, D. T. Chem. Rev. 2007, 107, 2300-2318. doi:10.1021/cr050944c

57. Lee, C.-Y.; Chang, C.-L.; Wang, Y.-N.; Fu, L.-M. Int. J. Mol. Sci. 2011, 12, 3263-3287. doi:10.3390/ijms12053263

58. Carter, C. F.; Lange, H.; Ley, S. V.; Baxendale, I. R.; Wittkamp, B.; Goode, J. G.; Gaunt, N. L. Org. Process Res. Dev. 2010, 14, 393-404. doi:10.1021/op900305v

59. Perro, A.; Lebourdon, G.; Henry, S.; Lecomte, S.; Servant, L.; Marre, S. React. Chem. Eng. 2016, 1, 577-594. doi:10.1039/c6re00127k

60. Poh, J.-S.; Browne, D. L.; Ley, S. V. React. Chem. Eng. 2016, 1, 101-105. doi:10.1039/c5re00082c

61. Glotz, G.; Lebl, R.; Dallinger, D.; Kappe, C. O. Angew. Chem., Int. Ed. 2017, 56, 13786-13789. doi:10.1002/anie.201708533

62. Segal-Rosenheimer, M.; Dubowski, Y. J. Phys. Chem. C 2007, 111, 11682-11691. doi:10.1021/jp072937t

63. Giraudeau, P.; Felpin, F.-X. React. Chem. Eng. 2018, 3, 399-413. doi:10.1039/c8re00083b

64. Thomson, C. G.; Jones, C. M. S.; Rosair, G.; Ellis, D.; Marques-Hueso, J.; Lee, A.-L.; Vilela, F. J. Flow Chem. 2020, 10, 327-345. doi:10.1007/s41981-019-00067-4

65. Sagmeister, P.; Williams, J. D.; Hone, C. A.; Kappe, C. O. React. Chem. Eng. 2019, 4, 1571-1578. doi:10.1039/c9re00087a

66. Archambault, C. M.; Leadbeater, N. E. RSC Adv. 2016, 6, 101171-101177. doi:10.1039/c6ra19662d

67. Mancino, V.; Cerra, B.; Piccinno, A.; Gioiello, A. Org. Process Res. Dev. 2018, 22, 600-607. doi:10.1021/acs.oprd.8b00038

68. Hopkin, M. D.; Baxendale, I. R.; Ley, S. V. Chem. Commun. 2010, 46, 2450-2452. doi:10.1039/c001550d

69. Krishnadasan, S.; Brown, R. J. C.; deMello, A. J.; deMello, J. C. Lab Chip 2007, 7, 1434-1441. doi:10.1039/b711412e

70. Trunina, D.; Liauw, M. A. Chem. Ing. Tech. 2020, 92, 659-664. doi:10.1002/cite.201900145

71. Loren, B. P.; Wleklinski, M.; Koswara, A.; Yammine, K.; Hu, Y.; Nagy, Z. K.; Thompson, D. H.; Cooks, R. G. Chem. Sci. 2017, 8 , 4363-4370. doi:10.1039/c7sc00905d

72. Browne, D. L.; Wright, S.; Deadman, B. J.; Dunnage, S.; Baxendale, I. R.; Turner, R. M.; Ley, S. V.

Rapid Commun. Mass Spectrom. 2012, 26, 1999-2010. doi:10.1002/rcm.6312
73. Benz, C.; Boomhoff, M.; Appun, J.; Schneider, C.; Belder, D. Angew. Chem., Int. Ed. 2015, 54, 2766-2770. doi:10.1002/anie.201409663

74. Schwolow, S.; Braun, F.; Rädle, M.; Kockmann, N.; Röder, T. Org. Process Res. Dev. 2015, 19, 1286-1292. doi:10.1021/acs.oprd.5b00184

75. Mozharov, S.; Nordon, A.; Littlejohn, D.; Wiles, C.; Watts, P.; Dallin, P.; Girkin, J. M. J. Am. Chem. Soc. 2011, 133, 3601-3608. doi:10.1021/ja1102234

76. Hamlin, T. A.; Leadbeater, N. E. Beilstein J. Org. Chem. 2013, 9, 1843-1852. doi:10.3762/bjoc.9.215

77. Pelletier, M. J.; Fabiilli, M. L.; Moon, B. Appl. Spectrosc. 2007, 61, 1107-1115. doi:10.1366/000370207782217725

78. Baxendale, I. R.; Ley, S. V. Solid Supported Reagents in Multi-Step Flow Synthesis. In New Avenues to Efficient Chemical Synthesis; Seeberger, P. H.; Blume, T., Eds.; Ernst Schering Foundation Symposium Proceedings, Vol. 2006/3; Springer: Berlin, Heidelberg, 2007. doi:10.1007/2789_2007_033

79. Baumann, M.; Baxendale, I. R.; Ley, S. V.; Smith, C. D.; Tranmer, G. K. Org. Lett. 2006, 8, 5231-5234. doi:10.1021/ol061975c

80. Dräger, G.; Kiss, C.; Kunz, U.; Kirschning, A. Org. Biomol. Chem. 2007, 5, 3657-3664. doi:10.1039/b712804e

81. Baxendale, I. R.; Ley, S. V.; Smith, C. D.; Tranmer, G. K. Chem. Commun. 2006, 4835. doi:10.1039/b612197g

82. Smith, C. D.; Baxendale, I. R.; Tranmer, G. K.; Baumann, M.; Smith, S. C.; Lewthwaite, R. A.; Ley, S. V. Org. Biomol. Chem. 2007, 5, 1562-1568. doi:10.1039/b703033a

83. Smith, C. D.; Baxendale, I. R.; Lanners, S.; Hayward, J. J.; Smith, S. C.; Ley, S. V. Org. Biomol. Chem. 2007, 5, 1559-1561. doi:10.1039/b702995k

84. Baumann, M.; Baxendale, I. R.; Ley, S. V.; Nikbin, N.; Smith, C. D. Org. Biomol. Chem. 2008, 6, 1587-1593. doi:10.1039/b801634h

85. Baumann, M.; Baxendale, I. R.; Ley, S. V. Synlett 2008, 2111-2114. doi:10.1055/s-2008-1078026

86. Mallia, C. J.; Baxendale, I. R. Org. Process Res. Dev. 2016, 20, 327-360. doi:10.1021/acs.oprd.5b00222

87. Wiles, C.; Watts, P.; Haswell, S. J.; Pombo-Villar, E. Lab Chip 2001, 1, 100. doi:10.1039/b107861e

88. Kawaguchi, T.; Miyata, H.; Ataka, K.; Mae, K.; Yoshida, J.-i. Angew. Chem., Int. Ed. 2005, 44, 2413-2416. doi:10.1002/anie.200462466

89. Wiles, C.; Watts, P.; Haswell, S. J.; Pombo-Villar, E. Lab Chip 2004, 4, 171-173. doi:10.1039/b400280f

90. Hua, J.; Liu, X.; Xu, W.; An, L.; Zhang, J.; Wang, K.; Wan, M.; Zhang, F. Production Process of Anisyl Propionaldehyde. Chin. Patent CN103073403A, April 1, 2013.

91. Knorr, A.; Weissenborn, A. Manufacture of Para-Isopropyl-Alpha-Methyl Hydrocinnamic Aldehyde. U.S. Patent US1844013A, Sept 2, 1932.

92. Chapuis, C.; Skuy, D.; Richard, C.-A. Chimia 2019, 73, 194-204. doi:10.2533/chimia.2019.194

93. Mine, K.; Fukada, K. Process for Producing Cycloalkanone Derivatives. Eur. Patent EP1,433,773, June 30, 2004.

94. Stetter, H. P. D.; Kuhlmann, H. D. C. Verfahren Zur Herstellung von Ketonen. Ger. Patent DE2,437,219, Nov 11, 1976.

95. Pecunioso, A.; Menicagli, R. J. Org. Chem. 1988, 53, 2614-2617. doi:10.1021/jo00246a041

96. Tanaka, K.; Motomatsu, S.; Koyama, K.; Fukase, K. Tetrahedron Lett. 2008, 49, 2010-2012. doi:10.1016/j.tetlet.2008.01.057 
97. Tanaka, K.; Fukase, K. Org. Process Res. Dev. 2009, 13, 983-990. doi:10.1021/op900084f

98. Tanaka, K.; Motomatsu, S.; Koyama, K.; Tanaka, S.-i.; Fukase, K. Org. Lett. 2007, 9, 299-302. doi:10.1021/ol062777o

99. Doi, T.; Otaka, H.; Umeda, K.; Yoshida, M. Tetrahedron 2015, 71, 6463-6470. doi:10.1016/j.tet.2015.05.051

100.Gauthier, D. R., Jr.; Sherry, B. D.; Cao, Y.; Journet, M.; Humphrey, G.; Itoh, T.; Mangion, I.; Tschaen, D. M. Org. Lett. 2015, 17, 1353-1356. doi:10.1021/ol503625z

101. Mukherjee, S.; Yang, J. W.; Hoffmann, S.; List, B. Chem. Rev. 2007, 107, 5471-5569. doi:10.1021/cr0684016

102.Zhou, Q.-L. Angew. Chem., Int. Ed. 2016, 55, 5352-5353. doi:10.1002/anie.201509164

103.Odedra, A.; Seeberger, P. H. Angew. Chem., Int. Ed. 2009, 48, 2699-2702. doi:10.1002/anie.200804407

104.Schober, L.; Ratnam, S.; Yamashita, Y.; Adebar, N.; Pieper, M.; Berkessel, A.; Hessel, V.; Gröger, H. Synthesis 2019, 51, 1178-1184. doi:10.1055/s-0037-1610404

105. Munirathinam, R.; Huskens, J.; Verboom, W. Adv. Synth. Catal. 2015, 357, 1093-1123. doi:10.1002/adsc.201401081

106.Bortolini, O.; Caciolli, L.; Cavazzini, A.; Costa, V.; Greco, R.; Massi, A.; Pasti, L. Green Chem. 2012, 14, 992-1000. doi:10.1039/c2gc16673a

107.Ayats, C.; Henseler, A. H.; Pericàs, M. A. ChemSusChem 2012, 5 , 320-325. doi:10.1002/cssc.201100570

108.Ötvös, S. B.; Mándity, I. M.; Fülöp, F. J. Catal. 2012, 295, 179-185. doi:10.1016/j.jcat.2012.08.006

109. Nakashima, E.; Yamamoto, H. Chem. - Eur. J. 2018, 24, 1076-1079. doi:10.1002/chem.201705982

110.Brown, A. J.; Brunelli, N. A.; Eum, K.; Rashidi, F.; Johnson, J. R.; Koros, W. J.; Jones, C. W.; Nair, S. Science 2014, 345, 72-75. doi:10.1126/science. 1251181

111. Rezaei, F.; Lively, R. P.; Labreche, Y.; Chen, G.; Fan, Y.; Koros, W. J.; Jones, C. W. ACS Appl. Mater. Interfaces 2013, 5, 3921-3931. doi:10.1021/am400636c

112.Moschetta, E. G.; Negretti, S.; Chepiga, K. M.; Brunelli, N. A.; Labreche, Y.; Feng, Y.; Rezaei, F.; Lively, R. P.; Koros, W. J.; Davies, H. M. L.; Jones, C. W. Angew. Chem., Int. Ed. 2015, 54 , 6470-6474. doi:10.1002/anie.201500841

113.He, Y.; Jawad, A.; Li, X.; Atanga, M.; Rezaei, F.; Rownaghi, A. A. J. Catal. 2016, 341, 149-159. doi:10.1016/j.jcat.2016.07.001

114.Babich, L.; Hartog, A. F.; van Hemert, L. J. C.; Rutjes, F. P. J. T.; Wever, R. ChemSusChem 2012, 5, 2348-2353. doi:10.1002/cssc.201200468

115. Kikhtyanin, O.; Hora, L.; Kubička, D. Catal. Commun. 2015, 58, 89-92. doi:10.1016/j.catcom.2014.09.002

116. Kaizik, A.; Fridag, D.; Lueken, H. G.; Bueschken, W. Process for Preparing $\alpha, \beta$-Unsaturated C10-Aldehydes. U.S. Patent US8,581,008, Nov 12, 2013.

117.Bermann, D.; Frey, G. D.; Schalapski, K.; Strutz, H. Method for Carrying Out Multiphase Aldol Condensation Reactions to Give Mixed $\alpha, \beta$-Unsaturated Aldehydes. U.S Pat. Appl. US2014/0005441 A1, Jan 2, 2014.

118. Viviano, M.; Glasnov, T. N.; Reichart, B.; Tekautz, G.; Kappe, C. O. Org. Process Res. Dev. 2011, 15, 858-870. doi:10.1021/op2001047

119.Bonrath, W.; Pressel, Y.; Schütz, J.; Ferfecki, E.; Topp, K.-D. ChemCatChem 2016, 8, 3584-3591. doi:10.1002/cctc.201600867

120.Laroche, B.; Saito, Y.; Ishitani, H.; Kobayashi, S. Org. Process Res. Dev. 2019, 23, 961-967. doi:10.1021/acs.oprd.9b00048
121.Zhou, F.; Liu, H.; Wen, Z.; Zhang, B.; Chen, G. Ind. Eng. Chem. Res. 2018, 57, 11288-11298. doi:10.1021/acs.iecr.8b02367

122.Dobler, W.; Bahr, N.; Breuer, K.; Kindler, A. Continuous Process for Producing Pseudoionones and lonones. U.S. Patent US7141698B2, Nov 28, 2006.

123.Waldron, C.; Cao, E.; Cattaneo, S.; Brett, G. L.; Miedziak, P. J.; Wu, G.; Sankar, M.; Hutchings, G. J.; Gavriilidis, A. Chem. Eng. J. 2019, 377, 119976. doi:10.1016/j.cej.2018.09.137

124.Stevens, J. G.; Bourne, R. A.; Poliakoff, M. Green Chem. 2009, 11 , 409-416. doi:10.1039/b819687g

125. Chen, A. B.; Li, X.; Zhou, Y. Z.; Huang, L. L.; Fang, Z.; Gan, H. F.; Guo, K. Adv. Mater. Res. 2013, 781-784, 936-941. doi:10.4028/www.scientific.net/amr.781-784.936

126.Zak, J.; Ron, D.; Riva, E.; Harding, H. P.; Cross, B. C. S.; Baxendale, I. R. Chem. - Eur. J. 2012, 18, 9901-9910. doi:10.1002/chem.201201039

127. Alvarez-Casao, Y.; Marques-Lopez, E.; Herrera, R. P. Symmetry 2011, 3, 220-245. doi:10.3390/sym3020220

128. Milner, S. E.; Moody, T. S.; Maguire, A. R. Eur. J. Org. Chem. 2012, 3059-3067. doi:10.1002/ejoc.201101840

129. Luzzio, F. A. Tetrahedron 2001, 57, 915-945. doi:10.1016/s0040-4020(00)00965-0

130. Ballini, R.; Palmieri, A. Curr. Org. Chem. 2006, 10, 2145-2169. doi:10.2174/138527206778742632

131. Ballini, R.; Petrini, M.; Rosini, G. Molecules 2008, 13, 319-330. doi:10.3390/molecules13020319

132. Rosini, G.; Ballini, R.; Sorrenti, P. Tetrahedron 1983, 39, 4127-4132. doi:10.1016/s0040-4020(01)88632-4

133. Soldi, L.; Ferstl, W.; Loebbecke, S.; Maggi, R.; Malmassari, C.; Sartori, G.; Yada, S. J. Catal. 2008, 258, 289-295. doi:10.1016/j.jcat.2008.07.005

134. Biradar, A. V.; Sharma, K. K.; Asefa, T. Appl. Catal., A 2010, 389 , 19-26. doi:10.1016/j.apcata.2010.08.056

135. Lee, J.-W.; Mayer-Gall, T.; Opwis, K.; Song, C. E.; Gutmann, J. S.; List, B. Science 2013, 341, 1225-1229. doi:10.1126/science.1242196

136. Wang, L.; Xu, G.; Xiao, J.; Tao, M.; Zhang, W. Ind. Eng. Chem. Res. 2019, 58, 12401-12410. doi:10.1021/acs.iecr.9b01375

137. Ogawa, T.; Kumagai, N.; Shibasaki, M. Angew. Chem., Int. Ed. 2013, 52, 6196-6201. doi:10.1002/anie.201302236

138. Hashimoto, K.; Kumagai, N.; Shibasaki, M. Org. Lett. 2014, 16, 3496-3499. doi:10.1021/ol501432h

139. Nonoyama, A.; Hashimoto, K.; Saito, A.; Kumagai, N.; Shibasaki, M. Tetrahedron Lett. 2016, 57, 1815-1819. doi:10.1016/j.tetlet.2016.03.041

140. Karasawa, T.; Oriez, R.; Kumagai, N.; Shibasaki, M. J. Am. Chem. Soc. 2018, 140, 12290-12295. doi:10.1021/jacs.8b08236

141. Nonoyama, A.; Kumagai, N.; Shibasaki, M. Tetrahedron 2017, 73, 1517-1521. doi:10.1016/j.tet.2017.01.066

142.Parra-Cabrera, C.; Achille, C.; Kuhn, S.; Ameloot, R. Chem. Soc. Rev. 2018, 47, 209-230. doi:10.1039/c7cs00631d

143. Au, A. K.; Huynh, W.; Horowitz, L. F.; Folch, A. Angew. Chem., Int. Ed. 2016, 55, 3862-3881. doi:10.1002/anie.201504382

144. Neumaier, J. M.; Madani, A.; Klein, T.; Ziegler, T. Beilstein J. Org. Chem. 2019, 15, 558-566. doi:10.3762/bjoc.15.50

145. Rossi, S.; Porta, R.; Brenna, D.; Puglisi, A.; Benaglia, M. Angew. Chem., Int. Ed. 2017, 56, 4290-4294. doi:10.1002/anie.201612192 
146.Dai, Y.-f.; Tian, H.-y.; Sun, B.-g.; Sun, Y.-m.; Chen, H.-t.; Liu, X.-y. J. Chem. Res. 2012, 36, 495-496. doi:10.3184/174751912x13402955389383

147. Mane, J.; Clinet, J.-C. Substituted Octane(Ene) Nitriles, Methods for the Synthesis Thereof and Uses Thereof in Perfumery. U.S. Pat. Appl. US20100279917A1, Nov 4, 2010.

148.DeSimone, R. S. Process for the Preparation of $\alpha, \beta$-Unsaturated Nitriles. U.S. Pat. Appl. US3960923A, June 1, 1976.

149. Bonrath, W.; Medlock, J.; Lehmann, H.; Beumer, R.; Fischesser, J. Process for the Manufacture of Hydrogenated Nitriles. WO Pat. Appl. WO2015/024978A1, Feb 26, 2015.

150. Hopp, R.; Thielmann, T.; Gottsch, W. Cyclopentyl-Cyanomethyl-Cyclopentenes, Process for Their Preparation and Their Use as Odoriferous Substances. U.S. Pat. Appl. US5212153A, May 18, 1993.

151.Weiss, M.; Brunner, B.; Ebel, K.; Krause, W. rocess for the Preparation of Ethylgeranonitrile. U.S. Pat. Appl. US20100204083A1, Aug 12, 2010.

152.Johnson, F.; Paul, K. G.; Favara, D. Process for Preparing Methyl Jasmonate and Related Compounds. U.S. Pat. Appl. US4014919A, March 29, 1977.

153. Flachsmann, F.; Bachmann, J.-P. Cycloalkylidene-(Ortho Substituted Phenyl)-Acetonitriles and Their Use as Odorants. U.S. Patent US7655701B2, Feb 2, 2010.

154. Angeletti, E.; Canepa, C.; Martinetti, G.; Venturello, P. J. Chem. Soc., Perkin Trans. 1 1989, 105-107. doi:10.1039/p19890000105

155.Xu, D. Z.; Shi, S.; Wang, Y. RSC Adv. 2013, 3, 23075-23079. doi:10.1039/c3ra43921f

156.Lai, S. M.; Ng, C. P.; Martin-Aranda, R.; Yeung, K. L. Microporous Mesoporous Mater. 2003, 66, 239-252. doi:10.1016/j.micromeso.2003.09.014

157.Sylla, M.; Joseph, D.; Chevallier, E.; Camara, C.; Dumas, F. Synthesis 2006, 1045-1049. doi:10.1055/s-2006-926344

158.Zhang, G.; Zhang, X.; Lv, J.; Liu, H.; Qu, J.; Yeung, K. L. Catal. Today 2012, 193, 221-225. doi:10.1016/j.cattod.2012.04.008

159.Zhang, G.; Zhang, T.; Zhang, X.; Yeung, K. L. Catal. Commun. 2015, 68, 93-96. doi:10.1016/j.catcom.2015.05.008

160.Jackson, T.; Clark, J. H.; Macquarrie, D. J.; Brophy, J. H. Green Chem. 2004, 6, 193-195. doi:10.1039/b315764b

161. Fletcher, P. D. I.; Haswell, S. J.; Pombo-Villar, E.; Warrington, B. H.; Watts, P.; Wong, S. Y. F.; Zhang, X. Tetrahedron 2002, 58, 4735-4757. doi:10.1016/s0040-4020(02)00432-5

162. Nikbin, N.; Watts, P. Org. Process Res. Dev. 2004, 8, 942-944. doi:10.1021/op049857x

163. Vivier, D.; Soussia, I. B.; Rodrigues, N.; Lolignier, S.; Devilliers, M.; Chatelain, F. C.; Prival, L.; Chapuy, E.; Bourdier, G.; Bennis, K.; Lesage, F.; Eschalier, A.; Busserolles, J.; Ducki, S. J. Med. Chem. 2017, 60, 1076-1088. doi:10.1021/acs.jmedchem.6b01285

164.dos Anjos, J. V.; Srivastava, R. M.; Costa-Silva, J. H.; Scotti, L.; Scotti, M. T.; Wanderley, A. G.; Leite, E. S.; de Melo, S. J.; Mendonça Junior, F. J. B. Molecules 2012, 17, 809-819. doi:10.3390/molecules 17010809

165.Wang, Y.; Shi, D.; Tao, S.; Song, W.; Wang, H.; Wang, X.; Li, G.; Qiu, J.; Ji, M. ACS Sustainable Chem. Eng. 2016, 4, 1602-1610. doi:10.1021/acssuschemeng.5b01541

166.Song, W.; Shi, D.; Tao, S.; Li, Z.; Wang, Y.; Yu, Y.; Quu, J.; Ji, M.; Wang, X. J. Colloid Interface Sci. 2016, 481, 100-106. doi:10.1016/j.jcis.2016.07.052
167.Long, L.-Y.; Weng, Y.-X.; Wang, Y.-Z. Polymers (Basel, Switz.) 2018, 10, 623. doi:10.3390/polym10060623

168. Tsutsumi, Y.; Koga, H.; Qi, Z.-D.; Saito, T.; Isogai, A. Biomacromolecules 2014, 15, 4314-4319. doi:10.1021/bm501320b

169. Qiao, Y.; Teng, J.; Wang, S.; Ma, H. Molecules 2017, 23, 43. doi:10.3390/molecules23010043

170.Xu, G.; Jin, M.; Kalkhajeh, Y. K.; Wang, L.; Tao, M.; Zhang, W. J. Cleaner Prod. 2019, 231, 77-86. doi:10.1016/j.jclepro.2019.05.211

171.Zhu, H.; Xu, G.; Du, H.; Zhang, C.; Ma, N.; Zhang, W. J. Catal. 2019, 374, 217-229. doi:10.1016/j.jcat.2019.04.040

172. Ishitani, H.; Kanai, K.; Saito, Y.; Tsubogo, T.; Kobayashi, S. Eur. J. Org. Chem. 2017, 6491-6494. doi:10.1002/ejoc.201700998

173. Crawford, D. E.; Miskimmin, C. K. G.; Albadarin, A. B.; Walker, G.; James, S. L. Green Chem. 2017, 19, 1507-1518. doi:10.1039/c6gc03413f

174. Crawford, D. E.; Miskimmin, C. K.; Cahir, J.; James, S. L. Chem. Commun. 2017, 53, 13067-13070. doi:10.1039/c7cc06010f

175.Sharma, B. M.; Atapalkar, R. S.; Kulkarni, A. A. Green Chem. 2019, 21, 5639-5646. doi:10.1039/c9gc02447f

176.Aziridines and Epoxides in Organic Synthesis; Yudin, A. K., Ed.; Wiley-VCH: Weinheim, Germany, 2006. doi:10.1002/3527607862

177. de los Santos, J. M.; Ochoa de Retana, A. M.; Martínez de Marigorta, E.; Vicario, J.; Palacios, F. ChemCatChem 2018, 10, 5092-5114. doi:10.1002/cctc.201801026

178. Rotstein, B. H.; Zaretsky, S.; Rai, V.; Yudin, A. K. Chem. Rev. 2014, 114, 8323-8359. doi:10.1021/cr400615v

179. Brechtelsbauer, C.; Lewis, N.; Oxley, P.; Ricard, F.; Ramshaw, C. Org. Process Res. Dev. 2001, 5, 65-68. doi:10.1021/op0000834

180. Michael, A. Am. Chem. J. 1887, 9, 112.

181.Stork, G.; Brizzolara, A.; Landesman, H.; Szmuszkovicz, J.; Terrell, R. J. Am. Chem. Soc. 1963, 85, 207-222. doi:10.1021/ja00885a021

182. Thweatt, J. G. Preparation of Hydrocoumarin, Coumarin, and Alkyl Substituted Derivatives. U.S. Pat. Appl. US3442910A, May 6, 1969.

183. Wilson, R. A.; Mookherjee, B. D.; Hall, J. B.; Stork, G. Process for the Preparation of Methyl Dihydrojasmonate and Lower Alkyl Homologues. U.S. Pat. Appl. US4260830A, April 7, 1981.

184. Cohen, A. M. Proces for the Preparation of 3-Mono-Alkyl and 3,6-Dialkyl-Resorcylic Esters. Brit. Pat. Appl. GB1416040A, Dec 3, 1975.

185. Strotman, N. A.; Tan, Y.; Powers, K. W.; Soumeillant, M.; Leung, S. W. Org. Process Res. Dev. 2018, 22, 721-727. doi:10.1021/acs.oprd.8b00100

186. Christoffers, J.; Baro, A. Angew. Chem., Int. Ed. 2003, 42, 1688-1690. doi:10.1002/anie.200201614

187.Zheng, K.; Liu, X.; Feng, X. Chem. Rev. 2018, 118, 7586-7656. doi:10.1021/acs.chemrev.7b00692

188. Christoffers, J.; Koripelly, G.; Rosiak, A.; Rössle, M. Synthesis 2007, 1279-1300. doi:10.1055/s-2007-966005

189. Hayashi, M.; Matsubara, R. Tetrahedron Lett. 2017, 58, 1793-1805. doi:10.1016/j.tetlet.2017.03.044

190. Hattori, H. Chem. Rev. 1995, 95, 537-558. doi:10.1021/cr00035a005

191. Borah, P.; Yamashita, Y.; Kobayashi, S. Angew. Chem., Int. Ed. 2017, 56, 10330-10334. doi:10.1002/anie.201701789

192. Tsubogo, T.; Yamashita, Y.; Kobayashi, S. Chem. - Eur. J. 2012, 18, 13624-13628. doi:10.1002/chem.201202896

193. Tsubogo, T.; Oyamada, H.; Kobayashi, S. Nature 2015, 520, 329-332. doi:10.1038/nature14343

194.Bandini, M.; Fagioli, M.; Umani-Ronchi, A. Adv. Synth. Catal. 2004, 346, 545-548. doi:10.1002/adsc.200303213 
195. Melchiorre, P.; Marigo, M.; Carlone, A.; Bartoli, G. Angew. Chem., Int. Ed. 2008, 47, 6138-6171. doi:10.1002/anie.200705523

196. Mondal, A.; Bhowmick, S.; Ghosh, A.; Chanda, T.; Bhowmick, K. C. Tetrahedron: Asymmetry 2017, 28, 849-875. doi:10.1016/j.tetasy.2017.05.011

197. Atodiresei, I.; Vila, C.; Rueping, M. ACS Catal. 2015, 5, 1972-1985. doi:10.1021/acscatal.5b00002

198. Tůma, J.; Kohout, M. RSC Adv. 2018, 8, 1174-1181. doi:10.1039/c7ra12658a

199.Scatena, G. S.; de la Torre, A. F.; Cass, Q. B.; Rivera, D. G.; Paixão, M. W. ChemCatChem 2014, 6, 3208-3214. doi:10.1002/cctc.201402501

200. Arakawa, Y.; Wennemers, H. ChemSusChem 2013, 6, 242-245. doi:10.1002/cssc.201200740

201. Osorio-Planes, L.; Rodríguez-Escrich, C.; Pericàs, M. A. Catal. Sci. Technol. 2016, 6, 4686-4689. doi:10.1039/c6cy00473c

202. Kasaplar, P.; Rodríguez-Escrich, C.; Pericàs, M. A. Org. Lett. 2013, 15, 3498-3501. doi:10.1021/ol400974z

203. Izquierdo, J.; Ayats, C.; Henseler, A. H.; Pericàs, M. A. Org. Biomol. Chem. 2015, 13, 4204-4209. doi:10.1039/c5ob00325c

204.Sagamanova, I.; Rodríguez-Escrich, C.; Molnár, I. G.; Sayalero, S.; Gilmour, R.; Pericàs, M. A. ACS Catal. 2015, 5, 6241-6248. doi:10.1021/acscatal.5b01746

205.Du, J.; Shuai, B.; Tao, M.; Wang, G.; Zhang, W. Green Chem. 2016, 18, 2625-2631. doi:10.1039/c5gc02622a

206.Ötvös, S. B.; Pericàs, M. A.; Kappe, C. O. Chem. Sci. 2019, 10, 11141-11146. doi:10.1039/c9sc04752b

207. Ogasawara, S.; Hayashi, Y. Synthesis 2017, 49, 424-428. doi:10.1055/s-2016-0036-1588899

208. Hayashi, Y.; Ogasawara, S. Org. Lett. 2016, 18, 3426-3429. doi:10.1021/acs.orglett.6b01595

209.Du, L.-H.; Ling, H.-M.; Luo, X.-P. RSC Adv. 2014, 4, 7770-7773. doi:10.1039/c3ra47470d

210.Jadhav, A. S.; Anand, R. V. Eur. J. Org. Chem. 2017, 3716-3721. doi:10.1002/ejoc.201700587

211.Jadhav, A. S.; Anand, R. V. Org. Biomol. Chem. 2017, 15, 56-60. doi:10.1039/c6ob02277d

212. Murray, P. R. D.; Browne, D. L.; Pastre, J. C.; Butters, C.; Guthrie, D.; Ley, S. V. Org. Process Res. Dev. 2013, 17, 1192-1208. doi:10.1021/op4001548

213.Feringa, B. L.; Badorrey, R.; Pena, D.; Harutyunyan, S. R.; Minnaard, A. J. Proc. Natl. Acad. Sci. U. S. A. 2004, 101, 5834-5838. doi:10.1073/pnas.0308008101

214.Bonrath, W.; Crevatin, A.; Aquino, F. Process for the Preparation of Methylheptenone. WO2005075400A1, Aug 18, 2005.

215.Campbell, K. N.; Eby, L. T. J. Am. Chem. Soc. 1941, 63, 2683-2685. doi:10.1021/ja01855a050

216. Vani, P. V. S. N.; Chida, A. S.; Srinivasan, R.; Chandrasekharam, M.; Singh, A. K. Synth. Commun. 2001, 31, 219-224. doi:10.1081/scc-100000202

217. McMurry, J. E.; Melton, J. J. Am. Chem. Soc. 1971, 93, 5309-5311. doi:10.1021/ja00749a086

218.Buchi, G.; Egger, B. J. Org. Chem. 1971, 36, 2021-2023. doi:10.1021/jo00813a045

219.Selka, A.; Levesque, N. A.; Foucher, D.; Clarisse, O.; Chemat, F.; Touaibia, M. Org. Process Res. Dev. 2017, 21, 60-64. doi:10.1021/acs.oprd.6b00344

220. Hall, J. B.; Wiegers, W. J. Process and Product Produced by Said Process. U.S. Pat. Appl. US4014944A, March 29, 1977.
221. Gralla, G.; Heydrich, G.; Bergner, E. J.; Ebel, K. Process for Preparation of Menthol by Hydrogenation of Isopulegol. U.S. Patent US7.960,593B2, June 14, 2011.

222. Manuale, D. L.; Betti, C.; Marchi, A. J.; Yori, J. C.; Romeo, E. Quim. Nova 2010, 33, 1231-1234. doi:10.1590/s0100-40422010000600002

223. Cossar, P. J.; Hizartzidis, L.; Simone, M. I.; McCluskey, A.; Gordon, C. P. Org. Biomol. Chem. 2015, 13, 7119-7130. doi:10.1039/c5ob01067e

224. Abdel-Hamid, M. K.; Macgregor, K. A.; Odell, L. R.; Chau, N.; Mariana, A.; Whiting, A.; Robinson, P. J.; McCluskey, A. Org. Biomol. Chem. 2015, 13, 8016-8028. doi:10.1039/c5ob00751h

225. Fiasella, A.; Nuzzi, A.; Summa, M.; Armirotti, A.; Tarozzo, G.; Tarzia, G.; Mor, M.; Bertozzi, F.; Bandiera, T.; Piomelli, D. ChemMedChem 2014, 9, 1602-1614. doi:10.1002/cmdc.201300546

226. Baumann, M.; Baxendale, I. R.; Deplante, F. Beilstein J. Org. Chem. 2017, 13, 2549-2560. doi:10.3762/bjoc.13.251

227. Fabry, D. C.; Heddrich, S.; Sugiono, E.; Liauw, M. A.; Rueping, M. React. Chem. Eng. 2019, 4, 1486-1491. doi:10.1039/c9re00032a

228. Rossi, S.; Puglisi, A.; Benaglia, M. ChemCatChem 2018, 10 , 1512-1525. doi:10.1002/cctc.201701619

229.Silm, E.; Kaabel, S.; Järving, I.; Kanger, T. Synthesis 2019, 51, 4198-4204. doi:10.1055/s-0039-1690484

230. Boichenko, M. A.; Babkin, I. Y.; Kobylskoy, S. G.; Chagarovskiy, A. O.; Ivanova, O. A.; Trushkov, I. V. Molbank 2019, M1061. doi:10.3390/m1061

231.Szabó, B.; Tamás, B.; Faigl, F.; Éles, J.; Greiner, I. J. Flow Chem. 2019, 9, 13-17. doi:10.1007/s41981-018-00028-3

232.Sharma, Y.; Nikam, A. V.; Kulkarni, A. A. Org. Process Res. Dev. 2019, 23, 170-176. doi:10.1021/acs.oprd.8b00008

233.Szabó, B.; Szakter, K.; Thurner, A.; Faigl, F.; Éles, J.; Greiner, I. Period. Polytech., Chem. Eng. 2020, 64, 1-8. doi:10.3311/ppch.14275

234.Carter, C. F.; Lange, H.; Sakai, D.; Baxendale, I. R.; Ley, S. V. Chem. - Eur. J. 2011, 17, 3398-3405. doi:10.1002/chem.201003148

235. Chen, Z.; Mitchell, S.; Krumeich, F.; Hauert, R.; Yakunin, S.; Kovalenko, M. V.; Pérez-Ramírez, J. ACS Sustainable Chem. Eng. 2019, 7, 5223-5230. doi:10.1021/acssuschemeng.8b06148

236. Büchele, S.; Chen, Z.; Mitchell, S.; Hauert, R.; Krumeich, F.; Pérez-Ramírez, J. ChemCatChem 2019, 11, 2812-2820. doi:10.1002/cctc.201900547

237.Chen, Z.; Vorobyeva, E.; Mitchell, S.; Fako, E.; López, N.; Collins, S. M.; Leary, R. K.; Midgley, P. A.; Hauert, R.; Pérez-Ramírez, J. Natl. Sci. Rev. 2018, 5, 642-652. doi:10.1093/nsr/nwy048

238. Albani, D.; Shahrokhi, M.; Chen, Z.; Mitchell, S.; Hauert, R.; López, N.; Pérez-Ramírez, J. Nat. Commun. 2018, 9, 2634. doi:10.1038/s41467-018-05052-4

239. Coetzee, J.; Manyar, H. G.; Hardacre, C.; Cole-Hamilton, D. J. ChemCatChem 2013, 5, 2843-2847. doi:10.1002/cctc.201300431

240.Schmölzer, C.; Nowikow, C.; Kählig, H.; Schmid, W. Carbohydr. Res. 2013, 367, 1-4. doi:10.1016/j.carres.2012.11.019

241.Tran, K. T.; Pallesen, J. S.; Solbak, S. M. Ø.; Narayanan, D.; Baig, A.; Zang, J.; Aguayo-Orozco, A.; Carmona, R. M. C.; Garcia, A. D.; Bach, A. J. Med. Chem. 2019, 62, 8028-8052. doi:10.1021/acs.jmedchem.9b00723

242. Sharma, S.; Yamini, Y.; Das, P. New J. Chem. 2019, 43, 1764-1769. doi:10.1039/c8nj04646h 
243. Manzoni, L.; Belvisi, L.; Bianchi, A.; Conti, A.; Drago, C.; de Matteo, M.; Ferrante, L.; Mastrangelo, E.; Perego, P.; Potenza, D.; Scolastico, C.; Servida, F.; Timpano, G.; Vasile, F.; Rizzo, V.; Seneci, P. Bioorg. Med. Chem. 2012, 20, 6687-6708. doi:10.1016/j.bmc.2012.09.020

244.De Vita, E.; Smits, N.; van den Hurk, H.; Beck, E. M.; Hewitt, J.; Baillie, G.; Russell, E.; Pannifer, A.; Hamon, V.; Morrison, A.; McElroy, S. P.; Jones, P.; Ignatenko, N. A.; Gunkel, N.; Miller, A. K. ChemMedChem 2020, 15, 79-95. doi:10.1002/cmdc.201900536

245.Bryan, M. C.; Wernick, D.; Hein, C. D.; Petersen, J. V.; Eschelbach, J. W.; Doherty, E. M. Beilstein J. Org. Chem. 2011, 7, 1141-1149. doi:10.3762/bjoc.7.132

246. Bryan, M. C.; Hein, C. D.; Gao, H.; Xia, X.; Eastwood, H.; Bruenner, B. A.; Louie, S. W.; Doherty, E. M. ACS Comb. Sci. 2013, 15, 503-511. doi:10.1021/co400078r

247.Gintner, M.; Yoneda, Y.; Schmölzer, C.; Denner, C.; Kählig, H.; Schmid, W. Carbohydr. Res. 2019, 474, 34-42. doi:10.1016/j.carres.2019.01.009

248. Rattray, N. J. W.; Zalloum, W. A.; Mansell, D.; Latimer, J.; Jaffar, M.; Bichenkova, E. V.; Freeman, S. Tetrahedron 2013, 69, 2758-2766. doi:10.1016/j.tet.2013.01.086

249. Cooper, C. G. F.; Lee, E. R.; Silva, R. A.; Bourque, A. J.; Clark, S.; Katti, S.; Nivorozhkin, V. Org. Process Res. Dev. 2012, 16, 1090-1097. doi:10.1021/op2001222

250.Bana, P.; Szigetvári, Á.; Kóti, J.; Éles, J.; Greiner, I. React. Chem. Eng. 2019, 4, 652-657. doi:10.1039/c8re00266e

251.Ponomarev, K. Y.; Suslov, E. V.; Zakharenko, A. L.; Zakharova, O. D.; Rogachev, A. D.; Korchagina, D. V.; Zafar, A.; Reynisson, J.; Nefedov, A. A.; Volcho, K. P.; Salakhutdinov, N. F.; Lavrik, O. I. Bioorg. Chem. 2018, 76, 392-399. doi:10.1016/j.bioorg.2017.12.005

252. Liu, J.; Fitzgerald, A.; Mani, N. Synthesis 2012, 44, 2469-2473. doi:10.1055/s-0032-1316550

253.Baumann, M.; Baxendale, I. R.; Kuratli, C.; Ley, S. V.; Martin, R. E.; Schneider, J. ACS Comb. Sci. 2011, 13, 405-413. doi:10.1021/co2000357

254. Escribà-Gelonch, M.; de Leon Izeppi, G. A.; Kirschneck, D.; Hessel, V. ACS Sustainable Chem. Eng. 2019, 7, 17237-17251. doi:10.1021/acssuschemeng.9b03931

255.Tukacs, J. M.; Sylvester, Á.; Kmecz, I.; Jones, R. V.; Óvári, M.; Mika, L. T. R. Soc. Open Sci. 2019, 6, 182233. doi: $10.1098 /$ rsos. 182233

256. Cova, C. M.; Zuliani, A.; Muñoz-Batista, M. J.; Luque, R. Green Chem. 2019, 21, 4712-4722. doi:10.1039/c9gc01596e

257.Cova, C. M.; Zuliani, A.; Manno, R.; Sebastian, V.; Luque, R. Green Chem. 2020, 22, 1414-1423. doi:10.1039/c9gc04091a

258. Polyzos, A.; O'Brien, M.; Petersen, T. P.; Baxendale, I. R.; Ley, S. V. Angew. Chem., Int. Ed. 2011, 50, 1190-1193. doi:10.1002/anie.201006618

259. Mercadante, M. A.; Kelly, C. B.; (Xiang) Lee, C.; Leadbeater, N. E. Org. Process Res. Dev. 2012, 16, 1064-1068. doi:10.1021/op300019w

260.Dalla-Vechia, L.; Reichart, B.; Glasnov, T.; Miranda, L. S. M.; Kappe, C. O.; de Souza, R. O. M. A. Org. Biomol. Chem. 2013, 11, 6806-6813. doi:10.1039/c3ob41464g

261.Glasnov, T. N.; Kappe, C. O. Adv. Synth. Catal. 2010, 352, 3089-3097. doi:10.1002/adsc.201000646

262. Baxendale, I. R.; Deeley, J.; Griffiths-Jones, C. M.; Ley, S. V.; Saaby, S.; Tranmer, G. K. Chem. Commun. 2006, 2566. doi:10.1039/b600382f
263. Obermayer, D.; Glasnov, T. N.; Kappe, C. O. J. Org. Chem. 2011, 76, 6657-6669. doi:10.1021/j02009824

264.Brasholz, M.; Macdonald, J. M.; Saubern, S.; Ryan, J. H.; Holmes, A. B. Chem. - Eur. J. 2010, 16, 11471-11480. doi:10.1002/chem.201001435

265. O'Brien, M.; Taylor, N.; Polyzos, A.; Baxendale, I. R.; Ley, S. V. Chem. Sci. 2011, 2, 1250. doi:10.1039/c1sc00055a

266. Kobayashi, J.; Mori, Y.; Okamoto, K.; Akiyama, R.; Ueno, M.; Kitamori, T.; Kobayashi, S. Science 2004, 304, 1305-1308. doi:10.1126/science.1096956

267. Kobayashi, J.; Mori, Y.; Kobayashi, S. Chem. Commun. 2005, 2567-2568. doi:10.1039/b501169h

268.Bakker, J. J. W.; Zieverink, M. M. P.; Reintjens, R. W. E. G.; Kapteijn, F.; Moulijn, J. A.; Kreutzer, M. T. ChemCatChem 2011, 3, 1155-1157. doi:10.1002/cctc.201100044

269. Britton, J.; Stubbs, K. A.; Weiss, G. A.; Raston, C. L. Chem. - Eur. J. 2017, 23, 13270-13278. doi:10.1002/chem.201700888

270.Phillips, J. M.; Ahamed, M.; Duan, X.; Lamb, R. N.; Qu, X.; Zheng, K.; Zou, J.; Chalker, J. M.; Raston, C. L. ACS Appl. Bio Mater. 2019, 2, 488-494. doi:10.1021/acsabm.8b00678

271.Avril, A.; Hornung, C. H.; Urban, A.; Fraser, D.; Horne, M.; Veder, J.-P.; Tsanaktsidis, J.; Rodopoulos, T.; Henry, C.; Gunasegaram, D. R. React. Chem. Eng. 2017, 2, 180-188. doi:10.1039/c6re00188b

272.Hornung, C. H.; Nguyen, X.; Carafa, A.; Gardiner, J.; Urban, A.; Fraser, D.; Horne, M. D.; Gunasegaram, D. R.; Tsanaktsidis, J. Org. Process Res. Dev. 2017, 21, 1311-1319. doi:10.1021/acs.oprd.7b00180

273. Nguyen, X.; Carafa, A.; Hornung, C. H. Chem. Eng. Process. 2018, 124, 215-221. doi:10.1016/j.cep.2017.12.007

274. Gardiner, J.; Nguyen, X.; Genet, C.; Horne, M. D.; Hornung, C. H.; Tsanaktsidis, J. Org. Process Res. Dev. 2018, 22, 1448-1452. doi:10.1021/acs.oprd.8b00153

275. Takahashi, Y.; Mitsudome, T.; Mizugaki, T.; Jitsukawa, K.; Kaneda, K. Green Chem. 2013, 15, 2695. doi:10.1039/c3gc41322e

276. Russell, M. G.; Veryser, C.; Hunter, J. F.; Beingessner, R. L.; Jamison, T. F. Adv. Synth. Catal. 2020, 362, 314-319. doi:10.1002/adsc.201901185

277.Sachse, A.; Linares, N.; Barbaro, P.; Fajula, F.; Galarneau, A. Dalton Trans. 2013, 42, 1378-1384. doi:10.1039/c2dt31690k

278. Linares, N.; Moreno-Marrodan, C.; Barbaro, P. ChemCatChem 2016, 8, 1001-1011. doi:10.1002/cctc.201501126

279.Strohmann, M.; Bordet, A.; Vorholt, A. J.; Leitner, W. Green Chem. 2019, 21, 6299-6306. doi:10.1039/c9gc02555c

280.Lázaro, N.; Franco, A.; Ouyang, W.; Balu, A.; Romero, A.; Luque, R.; Pineda, A. Catalysts 2019, 9, 142. doi:10.3390/catal9020142

281. Ouyang, W.; Zhao, D.; Wang, Y.; Balu, A. M.; Len, C.; Luque, R. ACS Sustainable Chem. Eng. 2018, 6, 6746-6752. doi:10.1021/acssuschemeng.8b00549

282. Brandi, F.; Bäumel, M.; Molinari, V.; Shekova, I.; Lauermann, I.; Heil, T.; Antonietti, M.; Al-Naji, M. Green Chem. 2020, 22, 2755-2766. doi:10.1039/c9gc03826d

283. Hattori, T.; Ida, T.; Tsubone, A.; Sawama, Y.; Monguchi, Y.; Sajiki, H. Eur. J. Org. Chem. 2015, 2492-2497. doi:10.1002/ejoc.201500050

284. Miyamura, H.; Suzuki, A.; Yasukawa, T.; Kobayashi, S. J. Am. Chem. Soc. 2018, 140, 11325-11334. doi:10.1021/jacs.8b06015

285. Kobayashi, S.; Okumura, M.; Akatsuka, Y.; Miyamura, H.; Ueno, M.; Oyamada, H. ChemCatChem 2015, 7, 4025-4029. doi:10.1002/cctc.201500973 
286. Oyamada, H.; Naito, T.; Kobayashi, S. Beilstein J. Org. Chem. 2011, 7, 735-739. doi:10.3762/bjoc.7.83

287.Johnson, M. D.; May, S. A.; Calvin, J. R.; Remacle, J.; Stout, J. R.; Diseroad, W. D.; Zaborenko, N.; Haeberle, B. D.; Sun, W.-M.; Miller, M. T.; Brennan, J. Org. Process Res. Dev. 2012, 16, 1017-1038. doi:10.1021/op200362h

288. Meemken, F.; Baiker, A. Chem. Rev. 2017, 117, 11522-11569. doi:10.1021/acs.chemrev.7b00272

289.Ager, D. J.; de Vries, A. H. M.; de Vries, J. G. Chem. Soc. Rev. 2012, 41, 3340-3380. doi:10.1039/c2cs15312b

290.Sullivan, R. J.; Newman, S. G. Chem. Sci. 2018, 9, 2130-2134. doi:10.1039/c7sc05192a

291. Tripathi, S.; Singh, S. N.; Yadav, L. D. S. RSC Adv. 2016, 6, 14547-14551. doi:10.1039/c5ra26623h

292. Fritzsche. No Title. DE 207,702, 1909.

293. Kapelle, I. B. D.; Irawadi, T. T.; Rusli, M. S.; Mangunwidjaja, D.; Mas'ud, Z. A. Procedia Chem. 2015, 16, 480-488. doi:10.1016/j.proche.2015.12.082

294.Fisher, G. S.; Stinson, J. S.; Goldblatt, L. A. J. Am. Chem. Soc. 1953, 75, 3675-3678. doi:10.1021/ja01111a021

295.Wang, S.; Zhang, A. Org. Prep. Proced. Int. 2008, 40, 405-410. doi:10.1080/00304940809458102

296. Gavriilidis, A.; Constantinou, A.; Hellgardt, K.; Hii, K. K. (Mimi).; Hutchings, G. J.; Brett, G. L.; Kuhn, S.; Marsden, S. P. React. Chem. Eng. 2016, 1, 595-612. doi:10.1039/c6re00155f

297.Gemoets, H. P. L.; Su, Y.; Shang, M.; Hessel, V.; Luque, R.; Noël, T. Chem. Soc. Rev. 2016, 45, 83-117. doi:10.1039/c5cs00447k

298. Wang, N.; Matsumoto, T.; Ueno, M.; Miyamura, H.; Kobayashi, S. Angew. Chem., Int. Ed. 2009, 48, 4744-4746. doi:10.1002/anie.200900565

299. Osako, T.; Torii, K.; Uozumi, Y. RSC Adv. 2015, 5, 2647-2654. doi:10.1039/c4ra14947e

300. Mannel, D. S.; Stahl, S. S.; Root, T. W. Org. Process Res. Dev. 2014, 18, 1503-1508. doi:10.1021/op5002676

301.Zotova, N.; Hellgardt, K.; Kelsall, G. H.; Jessiman, A. S.; Hii, K. K. (Mimi). Green Chem. 2010, 12, 2157-2163. doi:10.1039/c0gc00493f

302. Kaizuka, K.; Lee, K.-Y.; Miyamura, H.; Kobayashi, S. J. Flow Chem. 2012, 2, 1-4. doi:10.1556/jfchem.2011.00014

303.Wu, G.; Constantinou, A.; Cao, E.; Kuhn, S.; Morad, M.; Sankar, M.; Bethell, D.; Hutchings, G. J.; Gavriilidis, A. Ind. Eng. Chem. Res. 2015, 54, 4183-4189. doi:10.1021/ie5041176

304.Dhakshinamoorthy, A.; Navalon, S.; Asiri, A. M.; Garcia, H. Chem. Commun. 2020, 56, 26-45. doi:10.1039/c9cc07953j

305. Vanoye, L.; Pablos, M.; Smith, N.; de Bellefon, C.; Favre-Réguillon, A. RSC Adv. 2014, 4, 57159-57163. doi:10.1039/c4ra12067a

306. Vanoye, L.; Aloui, A.; Pablos, M.; Philippe, R.; Percheron, A.; Favre-Réguillon, A.; de Bellefon, C. Org. Lett. 2013, 15, 5978-5981. doi:10.1021/ol401273k

307.Ötvös, S. B.; Llanes, P.; Pericàs, M. A.; Kappe, C. O. Org. Lett. 2020, 22, 8122-8126. doi:10.1021/acs.orglett.0c03100

308.Sedelmeier, J.; Ley, S. V.; Baxendale, I. R.; Baumann, M. Org. Lett. 2010, 12, 3618-3621. doi:10.1021/ol101345z

309.Bourne, S. L.; Ley, S. V. Adv. Synth. Catal. 2013, 355, 1905-1910. doi:10.1002/adsc.201300278

310.Burkholder, M.; Gilliland, S. E., III; Luxon, A.; Tang, C.; Gupton, B. F. Catalysts 2019, 9, 95. doi:10.3390/catal9010095

311.Pieber, B.; Kappe, C. O. Green Chem. 2013, 15, 320. doi:10.1039/c2gc36896j
312. Park, C. Y.; Park, J. H.; Lim, H. J.; Hwang, G.-S.; Park, C. P. Bull. Korean Chem. Soc. 2014, 35, 983-984. doi:10.5012/bkcs.2014.35.4.983

313.Park, C. Y.; Kim, Y. J.; Lim, H. J.; Park, J. H.; Kim, M. J.; Seo, S. W.; Park, C. P. RSC Adv. 2015, 5, 4233-4237. doi:10.1039/c4ra12965b

314. Maurya, R. A.; Park, C. P.; Kim, D.-P. Beilstein J. Org. Chem. 2011, 7, 1158-1163. doi:10.3762/bjoc.7.134

315.Lévesque, F.; Seeberger, P. H. Org. Lett. 2011, 13, 5008-5011. doi:10.1021/ol2017643

316. Hook, B. D. A.; Dohle, W.; Hirst, P. R.; Pickworth, M.; Berry, M. B.; Booker-Milburn, K. I. J. Org. Chem. 2005, 70, 7558-7564. doi:10.1021/jo050705p

317. Matsuura, S.-i.; Yokoyama, T.; Ishii, R.; Itoh, T.; Tomon, E.; Hamakawa, S.; Tsunoda, T.; Mizukami, F.; Nanbu, H.; Hanaoka, T.-a. Chem. Commun. 2012, 48, 7058. doi:10.1039/c2cc32271d

318. Cvjetko, M.; Vorkapić-Furač, J.; Žnidaršič-Plazl, P. Process Biochem. (Oxford, U. K.) 2012, 47, 1344-1350. doi:10.1016/j.procbio.2012.04.028

319. Illner, S.; Hofmann, C.; Löb, P.; Kragl, U. ChemCatChem 2014, 6, 1748-1754. doi:10.1002/cctc.201400028

320.Greene, J. F.; Hoover, J. M.; Mannel, D. S.; Root, T. W.; Stahl, S. S. Org. Process Res. Dev. 2013, 17, 1247-1251. doi:10.1021/op400207f

321. Ye, X.; Johnson, M. D.; Diao, T.; Yates, M. H.; Stahl, S. S. Green Chem. 2010, 12, 1180. doi:10.1039/c0gc00106f

322. Sharma, Y.; Moolya, S.; Joshi, R. A.; Kulkarni, A. A. React. Chem. Eng. 2017, 2, 304-308. doi:10.1039/c6re00225k

323. Bogdan, A.; McQuade, D. T. Beilstein J. Org. Chem. 2009, 5, No. 17. doi:10.3762/bjoc.5.17

324. Okuno, Y.; Kitagawa, Y.; Kamiya, S.; Hasegawa, A.; Kawashima, T.; Otani, K.; Aoki, S.; Kanno, J.; Isomura, S.; Sato, Y.; Takeda, K. Asian J. Org. Chem. 2018, 7, 1071-1074. doi:10.1002/ajoc.201800125

325.Schulze, J. S.; Migenda, J.; Becker, M.; Schuler, S. M. M.; Wende, R. C.; Schreiner, P. R.; Smarsly, B. M. J. Mater. Chem. A 2020, 8, 4107-4117. doi:10.1039/c9ta12416k

326. Aellig, C.; Scholz, D.; Conrad, S.; Hermans, I. Green Chem. 2013, 15, 1975-1980. doi:10.1039/c3gc40159f

327. Albanese, D. C. M.; Foschi, F.; Penso, M. Org. Process Res. Dev. 2016, 20, 129-139. doi:10.1021/acs.oprd.5b00385

328. Leduc, A. B.; Jamison, T. F. Org. Process Res. Dev. 2012, 16 , 1082-1089. doi:10.1021/op200118h

329. Kairouz, V.; Collins, S. K. J. Chem. Educ. 2018, 95, 1069-1072. doi:10.1021/acs.jchemed.7b00412

330.Zhang, Y.; Born, S. C.; Jensen, K. F. Org. Process Res. Dev. 2014, 18, 1476-1481. doi:10.1021/op500158h

331.Peer, M.; Weeranoppanant, N.; Adamo, A.; Zhang, Y.; Jensen, K. F. Org. Process Res. Dev. 2016, 20, 1677-1685. doi:10.1021/acs.oprd.6b00234

332. He, W.; Fang, Z.; Tian, Q.; Shen, W.; Guo, K. Ind. Eng. Chem. Res. 2016, 55, 1373-1379. doi:10.1021/acs.iecr.5b04187

333. He, W.; Fang, Z.; Tian, Q.; Ji, D.; Zhang, K.; Guo, K. Chem. Eng. Process. 2015, 96, 39-43. doi:10.1016/j.cep.2015.07.028

334.Cossar, P. J.; Baker, J. R.; Cain, N.; McCluskey, A. R. Soc. Open Sci. 2018, 5, 171190. doi:10.1098/rsos.171190

335. Vanoye, L.; Wang, J.; Pablos, M.; de Bellefon, C.; Favre-Réguillon, A. Catal. Sci. Technol. 2016, 6, 4724-4732. doi:10.1039/c6cy00309e

336. Vanoye, L.; Hamami, Z. E.; Wang, J.; de Bellefon, C.; Fongarland, P.; Favre-Réguillon, A. Eur. J. Lipid Sci. Technol. 2017, 119, 1600281. doi:10.1002/ejlt.201600281 
337.Wan, Y. S. S.; Chau, J. L. H.; Gavriilidis, A.; Yeung, K. L. Chem. Commun. 2002, 878-879. doi:10.1039/b108356b

338. Aigner, M.; Grosso-Giordano, N. A.; Okrut, A.; Zones, S.; Katz, A. React. Chem. Eng. 2017, 2, 842-851. doi:10.1039/c7re00076f

339. Wulff, H. P. Heterogeneous Catalysts for Olefin Epoxidation. U.S. Pat. Appl. US3829392A, Aug 13, 1974.

340.Dai, W.; Li, G.; Chen, B.; Wang, L.; Gao, S. Org. Lett. 2015, 17, 904-907. doi:10.1021/acs.orglett.5b00018

341.Dai, W.; Mi, Y.; Lv, Y.; Shang, S.; Li, G.; Chen, G.; Gao, S. Synthesis 2016, 48, 2653-2658. doi:10.1055/s-0035-1561955

342.Kee, S.-P.; Gavriilidis, A. Org. Process Res. Dev. 2009, 13, 941-951. doi:10.1021/op800276a

343.Wiles, C.; Hammond, M. J.; Watts, P. Beilstein J. Org. Chem. 2009, 5 , No. 27. doi:10.3762/bjoc.5.27

344. Wada, Y.; Schmidt, M. A.; Jensen, K. F. Ind. Eng. Chem. Res. 2006, 45, 8036-8042. doi:10.1021/ie060893p

345. Hübner, S.; Bentrup, U.; Budde, U.; Lovis, K.; Dietrich, T.; Freitag, A.; Küpper, L.; Jähnisch, K. Org. Process Res. Dev. 2009, 13, 952-960. doi:10.1021/op9000669

346.Steinfeldt, N.; Abdallah, R.; Dingerdissen, U.; Jähnisch, K. Org. Process Res. Dev. 2007, 11, 1025-1031. doi:10.1021/op7000673

347.Steinfeldt, N.; Bentrup, U.; Jähnisch, K. Ind. Eng. Chem. Res. 2010, 49, 72-80. doi:10.1021/ie900726s

348. Irfan, M.; Glasnov, T. N.; Kappe, C. O. Org. Lett. 2011, 13, 984-987. doi:10.1021/ol102984h

349.O'Brien, M.; Baxendale, I. R.; Ley, S. V. Org. Lett. 2010, 12, 1596-1598. doi:10.1021/ol100322t

350.Kendall, A. J.; Barry, J. T.; Seidenkranz, D. T.; Ryerson, A.; Hiatt, C.; Salazar, C. A.; Bryant, D. J.; Tyler, D. R. Tetrahedron Lett. 2016, 57, 1342-1345. doi:10.1016/j.tetlet.2016.02.042

351.Schiaffo, C. E.; Dussault, P. H. J. Org. Chem. 2008, 73, 4688-4690. doi:10.1021/j0800323x

352. Roydhouse, M. D.; Ghaini, A.; Constantinou, A.; Cantu-Perez, A.; Motherwell, W. B.; Gavriilidis, A. Org. Process Res. Dev. 2011, 15, 989-996. doi:10.1021/op200036d

353. Roydhouse, M. D.; Motherwell, W. B.; Constantinou, A.; Gavriilidis, A.; Wheeler, R.; Down, K.; Campbell, I. RSC Adv. 2013, 3, 5076. doi:10.1039/c3ra00125c

354.Ren, J.; He, S.; Ye, C.; Chen, G.; Sun, C. Chem. Eng. J. 2012, 210 , 374-384. doi:10.1016/j.cej.2012.09.011

355.Lundin, M. D.; Danby, A. M.; Akien, G. R.; Venkitasubramanian, P.; Martin, K. J.; Busch, D. H.; Subramaniam, B. AlChE J. 2017, 63, 2819-2826. doi:10.1002/aic.15630

356.Qi, B.; Yang, H.-Y.; Wang, Z.-Q. Chin. J. Chem. 2007, 25, 1082-1084. doi:10.1002/cjoc.200790201

357.Gallimore, P. J.; Griffiths, P. T.; Pope, F. D.; Reid, J. P.; Kalberer, M. J. Geophys. Res.: Atmos. 2017, 122, 4364-4377. doi:10.1002/2016jd026221

358. Porterfield, J. P.; Eibenberger, S.; Patterson, D.; McCarthy, M. C. Phys. Chem. Chem. Phys. 2018, 20, 16828-16834. doi:10.1039/c8cp02055h

359. Hone, C. A.; O'Kearney-McMullan, A.; Munday, R.; Kappe, C. O. ChemCatChem 2017, 9, 3298-3302. doi:10.1002/cctc.201700671

360.Borlinghaus, J.; Albrecht, F.; Gruhlke, M. C. H.; Nwachukwu, I. D.; Slusarenko, A. J. Molecules 2014, 19, 12591-12618. doi:10.3390/molecules 190812591

361. Albrecht, F.; Leontiev, R.; Jacob, C.; Slusarenko, A. Molecules 2017, 22, 770. doi:10.3390/molecules22050770
362.Silva, F.; Khokhar, S. S.; Williams, D. M.; Saunders, R.; Evans, G. J. S.; Graz, M.; Wirth, T. Angew. Chem., Int. Ed. 2018, 57, 12290-12293. doi:10.1002/anie.201808605

363.Block, E.; Ahmad, S.; Jain, M. K.; Crecely, R. W.; Apitz-Castro, R.; Cruz, M. R. J. Am. Chem. Soc. 1984, 106, 8295-8296. doi:10.1021/ja00338a049

364.Dong, B. S.; Mi, Y. Y.; Ho, M. S.; Jae, H. H.; Sang, H. L.; Yong, S. C. Process for Preparing Ajoene from Garlic. U.S. Pat. Appl. US20120282334A1, Nov 8, 2012.

365. Colomer, J. P.; Traverssi, M.; Oksdath-Mansilla, G. J. Flow Chem. 2020, 10, 123-138. doi:10.1007/s41981-019-00066-5

366. Doherty, S.; Knight, J. G.; Carroll, M. A.; Clemmet, A. R.; Ellison, J. R.; Backhouse, T.; Holmes, N.; Thompson, L. A.; Bourne, R. A. RSC Adv. 2016, 6, 73118-73131. doi:10.1039/c6ra11157b

367.Doherty, S.; Knight, J. G.; Carroll, M. A.; Ellison, J. R.; Hobson, S. J.; Stevens, S.; Hardacre, C.; Goodrich, P. Green Chem. 2015, 17, 1559-1571. doi:10.1039/c4gc01770f

368.Silva, F.; Baker, A.; Stansall, J.; Michalska, W.; Yusubov, M. S.; Graz, M.; Saunders, R.; Evans, G. J. S.; Wirth, T. Eur. J. Org. Chem. 2018, 2134-2137. doi:10.1002/ejoc.201800339

369. Laudadio, G.; Straathof, N. J. W.; Lanting, M. D.; Knoops, B.; Hessel, V.; Noël, T. Green Chem. 2017, 19, 4061-4066. doi:10.1039/c7gc01973d

370.Gu, C. A.; Gu, L. Continuous Oxidation Method for Preparing Hexanoic Acid by Using Nitric Acid Oxidation Octanol. Chin. Pat. Appl. CN103,787,861A, June 14, 2015.

371.Wang, Z.; Deng, J. Method and Equipment for Preparing Aldehyde or Ketone through Continuous Oxidization by Oxygen. Chin. Pat. Appl. CN104,130,112A, Nov 5, 2014.

372.Sharley, J. S.; Baxendale, I. R.; Espinos Ferri, E.; Collado Perez, A. M.; Fernandez Fernandez, I.; Quesada, J. S. Chemical Process of Preparing Dehydrohedione. WO Pat. Appl. WO2018183788A1, Oct 4, 2018.

373. Klein, E.; Farnow, H.; Rojahn, W. Justus Liebigs Ann. Chem. 1964, 675, 73-82. doi:10.1002/jlac.19646750110

374. Miyake, A.; Nishino, M.; Kondo, H. 5-Cyclohexadecen-1-on Verfahren zu dessen Herstellung und seine Verwendung als Riechstoff July 4, 1974.

375. Watanabe, S.; Ujihara, H.; Yamamoto, T.; Hagiwara, T. Production Process of Cyclohexenyl Methyl Ketones. Eur. Pat. Appl. EP1162190A2, Dec 12, 2001.

376. Serra, S. Molecules 2015, 20, 12817-12840. doi:10.3390/molecules200712817

377. Grachev, A. A.; Klochkov, A. O.; Shiryaev, V. I. Russ. J. Appl. Chem. 2012, 85, 629-638. doi:10.1134/s1070427212040167

378. Goldbach, M.; Danieli, E.; Perlo, J.; Kaptein, B.; Litvinov, V. M.; Blümich, B.; Casanova, F.; Duchateau, A. L. L. Tetrahedron Lett. 2016, 57, 122-125. doi:10.1016/j.tetlet.2015.11.077

379. Huck, L.; de la Hoz, A.; Díaz-Ortiz, A.; Alcázar, J. Org. Lett. 2017, 19, 3747-3750. doi:10.1021/acs.orglett.7b01590

380.Deng, Y.; Wei, X.-J.; Wang, X.; Sun, Y.; Noël, T. Chem. - Eur. J. 2019, 25, 14532-14535. doi:10.1002/chem.201904480

381. Riva, E.; Gagliardi, S.; Martinelli, M.; Passarella, D.; Vigo, D.; Rencurosi, A. Tetrahedron 2010, 66, 3242-3247. doi:10.1016/j.tet.2010.02.078

382. Hamlin, T. A.; Lazarus, G. M. L.; Kelly, C. B.; Leadbeater, N. E. Org. Process Res. Dev. 2014, 18, 1253-1258. doi:10.1021/op500190j 383. Hamlin, T. A.; Kelly, C. B.; Cywar, R. M.; Leadbeater, N. E. J. Org. Chem. 2014, 79, 1145-1155. doi:10.1021/jo402577n 
384. Kupracz, L.; Kirschning, A. Adv. Synth. Catal. 2013, 355, 3375-3380. doi:10.1002/adsc.201300614

385.Battilocchio, C.; Baxendale, I. R.; Biava, M.; Kitching, M. O.; Ley, S. V. Org. Process Res. Dev. 2012, 16, 798-810. doi:10.1021/op300084z

386. Battilocchio, C.; Guetzoyan, L.; Cervetto, C.; Di Cesare Mannelli, L.; Frattaroli, D.; Baxendale, I. R.; Maura, G.; Rossi, A.; Sautebin, L.; Biava, M.; Ghelardini, C.; Marcoli, M.; Ley, S. V. ACS Med. Chem. Lett. 2013, 4, 704-709. doi:10.1021/ml400079h

387.Korwar, S.; Amir, S.; Tosso, P. N.; Desai, B. K.; Kong, C. J.; Fadnis, S.; Telang, N. S.; Ahmad, S.; Roper, T. D.; Gupton, B. F. Eur. J. Org. Chem. 2017, 6495-6498. doi:10.1002/ejoc.201701002

388.de M. Muñoz, J.; Alcázar, J.; de la Hoz, A.; Díaz-Ortiz, Á.; Alonso de Diego, S.-A. Green Chem. 2012, 14, 1335-1341. doi:10.1039/c2gc35037h

389. Mateos, C.; Rincón, J. A.; Villanueva, J. Tetrahedron Lett. 2013, 54, 2226-2230. doi:10.1016/j.tetlet.2013.02.069

390.Williams, J. D.; Kerr, W. J.; Leach, S. G.; Lindsay, D. M. Angew. Chem., Int. Ed. 2018, 57, 12126-12130. doi:10.1002/anie.201807393

391.Zhang, C.-T.; Zhu, R.; Wang, Z.; Ma, B.; Zajac, A.; Smiglak, M.; Xia, C.-N.; Castle, S. L.; Wang, W.-L. RSC Adv. 2019, 9, 2199-2204. doi:10.1039/c8ra07447j

392.Pedersen, M. J.; Holm, T. L.; Rahbek, J. P.; Skovby, T.; Mealy, M. J.; Dam-Johansen, K.; Kiil, S. Org. Process Res. Dev. 2013, 17 , 1142-1148. doi:10.1021/op400069e

393.Brodmann, T.; Koos, P.; Metzger, A.; Knochel, P.; Ley, S. V. Org. Process Res. Dev. 2012, 16, 1102-1113. doi:10.1021/op200275d

394.Wiss, J.; Länzlinger, M.; Wermuth, M. Org. Process Res. Dev. 2005 , 9, 365-371. doi:10.1021/op049784h

395. Hellings, M.; Van den Kerkhof, T. Applying PAT in Chemical Process Development.

https://www.europeanpharmaceuticalreview.com/article/905/applyingpat-in-chemical-process-development/ (accessed June 5, 2020).

396. Odille, F. G. J.; Stenemyr, A.; Pontén, F. Org. Process Res. Dev. 2014, 18, 1545-1549. doi:10.1021/op500290x

397.Kopach, M. E.; Cole, K. P.; Pollock, P. M.; Johnson, M. D.; Braden, T. M.; Webster, L. P.; McClary Groh, J.; McFarland, A. D.; Schafer, J. P.; Adler, J. J.; Rosemeyer, M. Org. Process Res. Dev. 2016, 20, 1581-1592. doi:10.1021/acs.oprd.6b00131

398.Wu, J.; Yang, X.; He, Z.; Mao, X.; Hatton, T. A.; Jamison, T. F. Angew. Chem., Int. Ed. 2014, 53, 8416-8420. doi:10.1002/anie.201405014

399. He, Z.; Jamison, T. F. Angew. Chem., Int. Ed. 2014, 53, 3353-3357. doi:10.1002/anie.201310572

400.Maier, M. C.; Lebl, R.; Sulzer, P.; Lechner, J.; Mayr, T.; Zadravec, M.; Slama, E.; Pfanner, S.; Schmölzer, C.; Pöchlauer, P.; Kappe, C. O.; Gruber-Woelfler, H. React. Chem. Eng. 2019, 4, 393-401. doi:10.1039/c8re00278a

401.Deng, Q.; Shen, R.; Ding, R.; Zhang, L. Adv. Synth. Catal. 2014, 356, 2931-2936. doi:10.1002/adsc.201400560

402.Lixiong, Z.; Lin, D.; Shen, W. Method for Preparing Propargyl Alcohol by Using Micro-Structural Reactor. Chin. Pat. Appl. CN103896737A, July 2, 2014.

403. Watanabe, S.; Nakaya, N.; Akai, J.; Kanaori, K.; Harada, T. Org. Lett. 2018, 20, 2737-2740. doi:10.1021/acs.orglett.8b00945

404. Nowicki, J. Molecules 2000, 5, 1033-1050. doi:10.3390/50801033 405. Luettin, A.; Stritt, C. Process for the Preparation of B-lonones and Vitmain A, Vitamin A Derivatives, Carotenes and Carotenoids. U.S. Pat. Appl. US20100312016A1, Dec 9, 2010.
406. Schulte-Elte, K.-H.; Willhalm, B.; Gautchl, F. Cyclic C6 Ketones in Perfumes. U.S. Patent US4,147,672, April 3, 1979.

407.Shen, D. L.; He, X.; Guan, W.; Liu, Y. P.; Sun, B. G. Adv. Mater. Res. 2013, 781-784, 983-987.

doi:10.4028/www.scientific.net/amr.781-784.983

408. Blackmore, R. L. Production of Terpene Compounds. U.S. Pat. Appl. US3281485A, Oct 25, 1966.

409. Cantillo, D.; de Frutos, O.; Rincón, J. A.; Mateos, C.; Kappe, C. O. J. Org. Chem. 2014, 79, 8486-8490. doi:10.1021/jo5016757

410. Fabry, D. C.; Ronge, M. A.; Rueping, M. Chem. - Eur. J. 2015, 21 , 5350-5354. doi:10.1002/chem.201406653

411. Baxendale, I. R.; Lee, A.-L.; Ley, S. V. Synlett 2002, 0516-0518. doi:10.1055/s-2002-20483

412.Billaud, E. M. F.; Shahbazali, E.; Ahamed, M.; Cleeren, F.; Noël, T.; Koole, M.; Verbruggen, A.; Hessel, V.; Bormans, G. Chem. Sci. 2017, 8, 1251-1258. doi:10.1039/c6sc02933g

413.Sauks, J. M.; Mallik, D.; Lawryshyn, Y.; Bender, T.; Organ, M. Org. Process Res. Dev. 2014, 18, 1310-1314. doi:10.1021/op400026g

414.Shahbazali, E.; Spapens, M.; Kobayashi, H.; Ookawara, S.; Noël, T.; Hessel, V. Chem. Eng. J. 2015, 281, 144-154. doi:10.1016/j.cej.2015.06.020

415. Hessel, V.; Shahbazali, E.; Noël, T.; Zelentsov, S. Chem. Ing. Tech. 2014, 86, 2160-2179. doi:10.1002/cite.201400125

416. Maeda, H.; Nashihara, S.; Mukae, H.; Yoshimi, Y.; Mizuno, K. Res. Chem. Intermed. 2013, 39, 301-310. doi:10.1007/s11164-012-0650-6

417. Rincón, J. A.; Barberis, M.; González-Esguevillas, M.; Johnson, M. D.; Niemeier, J. K.; Sun, W.-M. Org. Process Res. Dev. 2011, 15, 1428-1432. doi:10.1021/op200162c

418. Egami, H.; Tamaoki, S.; Abe, M.; Ohneda, N.; Yoshimura, T.; Okamoto, T.; Odajima, H.; Mase, N.; Takeda, K.; Hamashima, Y. Org. Process Res. Dev. 2018, 22, 1029-1033. doi:10.1021/acs.oprd.8b00185

419. Kobayashi, H.; Driessen, B.; van Osch, D. J. G. P.; Talla, A.; Ookawara, S.; Noël, T.; Hessel, V. Tetrahedron 2013, 69, 2885-2890. doi:10.1016/j.tet.2013.02.038

420.Baxendale, I. R.; Hornung, C.; Ley, S. V.; de Mata Muñoz Molina, J.; Wikström, A. Aust. J. Chem. 2013, 66, 131-144. doi:10.1071/ch12365

421.Xin, Q.; Jindong, H.; Xiangyang, T.; Wentao, Z. Method for Preparing Ocimene from Thermo Isomeric Alpha-Pinene in Liquid Phase. Chin. Pat. Appl. CN104672049A, 2015.

422. Ouchi, T.; Mutton, R. J.; Rojas, V.; Fitzpatrick, D. E.; Cork, D. G.; Battilocchio, C.; Ley, S. V. ACS Sustainable Chem. Eng. 2016, 4, 1912-1916. doi:10.1021/acssuschemeng.6b00287

423. Funel, J.-A.; Abele, S. Angew. Chem., Int. Ed. 2013, 52, 3822-3863. doi:10.1002/anie.201201636

424. Fráter, G.; Müller, U.; Schröder, F. Tetrahedron: Asymmetry 2004, 15, 3967-3972. doi:10.1016/j.tetasy.2004.11.003

425. Ayyar, K. S.; Cookson, R. C.; Kagi, D. A. J. Chem. Soc., Perkin Trans. 1 1975, 1727-1736. doi:10.1039/p19750001727

426. Baxendale, I. R. Chem. Eng. Technol. 2015, 38, 1713-1716. doi:10.1002/ceat.201500255

427.Hornung, C. H.; Álvarez-Diéguez, M. Á.; Kohl, T. M.; Tsanaktsidis, J. Beilstein J. Org. Chem. 2017, 13, 120-126. doi:10.3762/bjoc.13.15

428. Filipponi, P.; Gioiello, A.; Baxendale, I. R. Org. Process Res. Dev. 2016, 20, 371-375. doi:10.1021/acs.oprd.5b00331

429. Hartman, R. L. Org. Process Res. Dev. 2012, 16, 870-887. doi:10.1021/op200348t 
430.Polster, C. S.; Cole, K. P.; Burcham, C. L.; Campbell, B. M.; Frederick, A. L.; Hansen, M. M.; Harding, M.; Heller, M. R.; Miller, M. T.; Phillips, J. L.; Pollock, P. M.; Zaborenko, N. Org. Process Res. Dev. 2014, 18, 1295-1309. doi:10.1021/op500204z

431. Hartman, R. L.; Naber, J. R.; Zaborenko, N.; Buchwald, S. L.; Jensen, K. F. Org. Process Res. Dev. 2010, 14, 1347-1357. doi:10.1021/op100154d

432.Seghers, S.; Lefevere, J.; Mullens, S.; De Vylder, A.; Thybaut, J. W.; Stevens, C. V. ChemSusChem 2018, 11, 1686-1693. doi:10.1002/cssc.201800362

433.Seghers, S.; Protasova, L.; Mullens, S.; Thybaut, J. W.; Stevens, C. V. Green Chem. 2017, 19, 237-248. doi:10.1039/c6gc02334g

434. He, W.; Fang, Z.; Yang, Z.; Ji, D.; Guo, K. RSC Adv. 2015, 5, 58798-58803. doi:10.1039/c5ra08264a

435.Sachse, A.; Hulea, V.; Finiels, A.; Coq, B.; Fajula, F.; Galarneau, A. J. Catal. 2012, 287, 62-67. doi:10.1016/j.jcat.2011.12.003

436. Ahrendt, K. A.; Borths, C. J.; MacMillan, D. W. C. J. Am. Chem. Soc. 2000, 122, 4243-4244. doi:10.1021/ja000092s

437. Austin, J. F.; MacMillan, D. W. C. J. Am. Chem. Soc. 2002, 124, 1172-1173. doi:10.1021/ja017255c

438. Chiroli, V.; Benaglia, M.; Cozzi, F.; Puglisi, A.; Annunziata, R.; Celentano, G. Org. Lett. 2013, 15, 3590-3593. doi:10.1021/ol401390z

439. Porta, R.; Benaglia, M.; Chiroli, V.; Coccia, F.; Puglisi, A. Isr. J. Chem. 2014, 54, 381-394. doi:10.1002/ijch.201300106

440. Chiroli, V.; Benaglia, M.; Puglisi, A.; Porta, R.; Jumde, R. P.; Mandoli, A. Green Chem. 2014, 16, 2798-2806. doi:10.1039/c4gc00031e

441.Altava, B.; Burguete, M. I.; García-Verdugo, E.; Luis, S. V.; Vicent, M. J. Green Chem. 2006, 8, 717-726. doi:10.1039/b603494b

442. Abele, S.; Höck, S.; Schmidt, G.; Funel, J.-A.; Marti, R.

Org. Process Res. Dev. 2012, 16, 1114-1120.

doi:10.1021/op200320w

443.Damm, M.; Glasnov, T. N.; Kappe, C. O. Org. Process Res. Dev. 2010, 14, 215-224. doi:10.1021/op900297e

444.Tsoung, J.; Wang, Y.; Djuric, S. W. React. Chem. Eng. 2017, 2, 458-461. doi:10.1039/c7re00058h

445. Nakashima, E.; Yamamoto, H. Chem. Commun. 2015, 51 , 12309-12312. doi:10.1039/c5cc03458b

446.Bai-cheng, F.; Xi-chao, H.; Tie-lin, W.; Jian-qiang, L.; Yan, J. ChemistrySelect 2020, 5, 952-956. doi:10.1002/slct.201903919

447. Karl, D.; Löwe, H. J. Flow Chem. 2017, 7, 9-12. doi:10.1556/1846.2016.00039

448. Misuk, V.; Mai, A.; Zhao, Y.; Heinrich, J.; Rauber, D.; Giannopoulos, K.; Löwe, H. J. Flow Chem. 2015, 5, 101-109. doi:10.1556/1846.2015.00011

449. Khadra, A.; Organ, M. G. J. Flow Chem. 2016, 6, 293-296. doi:10.1556/1846.2016.00034

450.Spare, L. K.; Harman, D. G.; Aldrich-Wright, J. R.; Nguyen, T. V.; Gordon, C. P. Adv. Synth. Catal. 2018, 360, 1209-1217. doi:10.1002/adsc.201701471

451.Martin, R. E.; Morawitz, F.; Kuratli, C.; Alker, A. M.; Alanine, A. I. Eur. J. Org. Chem. 2012, 47-52. doi:10.1002/ejoc.201101538

452.Lehmann, J.; Alzieu, T.; Martin, R. E.; Britton, R. Org. Lett. 2013, 15, 3550-3553. doi:10.1021/ol4013525

453. Yokozawa, S.; Ohneda, N.; Muramatsu, K.; Okamoto, T.; Odajima, H.; Ikawa, T.; Sugiyama, J.-i.; Fujita, M.; Sawairi, T.; Egami, H.; Hamashima, Y.; Egi, M.; Akai, S. RSC Adv. 2015, 5, 10204-10210. doi:10.1039/c4ra12428f
454.Bah, J.; Franzén, J. Chem. - Eur. J. 2014, 20, 1066-1072. doi:10.1002/chem.201304160

455. Nekkaa, I.; Palkó, M.; Mándity, I. M.; Fülöp, F. Beilstein J. Org. Chem. 2018, 14, 318-324. doi:10.3762/bjoc. 14.20

456. Nekkaa, I.; Palkó, M.; Mándity, I. M.; Miklós, F.; Fülöp, F. Eur. J. Org. Chem. 2018, 4456-4464. doi:10.1002/ejoc.201800682

457.Ötvös, S. B.; Fülöp, F. Catal. Sci. Technol. 2015, 5, 4926-4941. doi:10.1039/c5cy00523j

458.Bao, J.; Tranmer, G. K. Chem. Commun. 2015, 51, 3037-3044. doi:10.1039/c4cc09221j

459. Kappe, C. O.; Van der Eycken, E. Chem. Soc. Rev. 2010, 39, 1280-1290. doi:10.1039/b901973c

460.Johansson, J. R.; Beke-Somfai, T.; Said Stålsmeden, A.; Kann, N. Chem. Rev. 2016, 116, 14726-14768. doi:10.1021/acs.chemrev.6b00466

461. Haldón, E.; Nicasio, M. C.; Pérez, P. J. Org. Biomol. Chem. 2015, 13, 9528-9550. doi:10.1039/c5ob01457c

462.Banerji, B.; Chandrasekhar, K.; Killi, S. K.; Pramanik, S. K.; Uttam, P.; Sen, S.; Maiti, N. C. R. Soc. Open Sci. 2016, 3, 160090. doi:10.1098/rsos. 160090

463. Meščić, A.; Šalić, A.; Gregorić, T.; Zelić, B.; Raić-Malić, S. RSC Adv. 2017, 7, 791-800. doi:10.1039/c6ra25244c

464. Teci, M.; Tilley, M.; McGuire, M. A.; Organ, M. G. Org. Process Res. Dev. 2016, 20, 1967-1973. doi:10.1021/acs.oprd.6b00292

465.Sadler, S.; Sebeika, M. M.; Kern, N. L.; Bell, D. E.; Laverack, C. A.; Wilkins, D. J.; Moeller, A. R.; Nicolaysen, B. C.; Kozlowski, P. N.; Wiles, C.; Tinder, R. J.; Jones, G. B. J. Flow Chem. 2014, 4, 140-147. doi:10.1556/jfc-d-14-00019

466. Leforestier, B.; Vögtle, M. Synlett 2016, 27, 1957-1962. doi:10.1055/s-0035-1561659

467.Salvador, C. E. M.; Pieber, B.; Neu, P. M.; Torvisco, A.; Andrade, C. K. Z.; Kappe, C. O. J. Org. Chem. 2015, 80, 4590-4602. doi:10.1021/acs.joc.5b00445

468. Bédard, A.-C.; Santandrea, J.; Collins, S. K. J. Flow Chem. 2015, 5, 142-144. doi:10.1556/jfc-d-14-00042

469. Pan, S.; Yan, S.; Osako, T.; Uozumi, Y. ACS Sustainable Chem. Eng. 2017, 5, 10722-10734. doi:10.1021/acssuschemeng.7b02646

470.Wen, J.; Wu, K.; Yang, D.; Tian, J.; Huang, Z.; Filatov, A. S.; Lei, A.; Lin, X.-M. ACS Appl. Mater. Interfaces 2018, 10, 25930-25935. doi:10.1021/acsami.8b06927

471.Burilov, V. A.; Nurmukhametova, A. N.; Belov, R. N.; Mironova, D. A.; Vorob'ev, V. V.; Osin, Y. N.; Antipin, I. S. Russ. Chem. Bull. 2018, 67, 461-468. doi:10.1007/s11172-018-2093-8

472.De Angelis, S.; Franco, M.; Triminì, A.; González, A.; Sainz, R.; Degennaro, L.; Romanazzi, G.; Carlucci, C.; Petrelli, V.; de la Esperanza, A.; Goñi, A.; Ferritto, R.; Aceña, J. L.; Luisi, R.; Cid, M. B. Chem. - Asian J. 2019, 14, 3011-3018. doi:10.1002/asia.201900781

473. Manzano, J. S.; Weinstein, Z. B.; Sadow, A. D.; Slowing, I. I. ACS Catal. 2017, 7, 7567-7577. doi:10.1021/acscatal.7b02111

474.Pucci, A.; Albano, G.; Pollastrini, M.; Lucci, A.; Colalillo, M.; Oliva, F.; Evangelisti, C.; Marelli, M.; Santalucia, D.; Mandoli, A. Catalysts 2020, 10, 434. doi:10.3390/catal10040434

475. Cao, J.; Xu, G.; Li, P.; Tao, M.; Zhang, W. ACS Sustainable Chem. Eng. 2017, 5, 3438-3447. doi:10.1021/acssuschemeng.7b00103

476. Manneveau, M.; Tanii, S.; Gens, F.; Legros, J.; Chataigner, I. Org. Biomol. Chem. 2020, 18, 3481-3486. doi:10.1039/d0ob00582g 
477. Baumann, M.; Baxendale, I. R.; Ley, S. V. Synlett 2010, 749-752. doi:10.1055/s-0029-1219344

478.Grafton, M.; Mansfield, A. C.; Fray, M. J. Tetrahedron Lett. 2010, 51, 1026-1029. doi:10.1016/j.tetlet.2009.12.071

479. Cludius-Brandt, S.; Kupracz, L.; Kirschning, A. Beilstein J. Org. Chem. 2013, 9, 1745-1750. doi:10.3762/bjoc.9.201

480. Chandrasekhar, D.; Borra, S.; Kapure, J. S.; Shivaji, G. S.; Srinivasulu, G.; Maurya, R. A. Org. Chem. Front. 2015, 2, 1308-1312. doi:10.1039/c5qo00207a

481. Britton, J.; Jamison, T. F. Angew. Chem., Int. Ed. 2017, 56, 8823-8827. doi:10.1002/anie.201704529

482. Audubert, C.; Gamboa Marin, O. J.; Lebel, H. Angew. Chem., Int. Ed. 2017, 56, 6294-6297. doi:10.1002/anie.201612235

483. Comas-Barceló, J.; Blanco-Ania, D.; van den Broek, S. A. M. W.; Nieuwland, P. J.; Harrity, J. P. A.; Rutjes, F. P. J. T. Catal. Sci. Technol. 2016, 6, 4718-4723. doi:10.1039/c5cy02247a

484.Jamison, T. F.; Palde, P. B. Use of Azides in Synthesis. U.S. Pat. Appl. US20130225819A1, Aug 29, 2013.

485. Daisuke-shi, N.; Imagawa, T.; Hirokawa, K.; Fumitaka, M. Method for Producing $1 \mathrm{H}$-Tetrazole Derivative. WO Pat. Appl. WO2017131213A1, Aug 3, 2017.

486. Castellano, S.; Tamborini, L.; Viviano, M.; Pinto, A.; Sbardella, G.; Conti, P. J. Org. Chem. 2010, 75, 7439-7442. doi:10.1021/j01014323

487.Brasholz, M.; Saubern, S.; Savage, G. P. Aust. J. Chem. 2011, 64, 1397. doi:10.1071/ch11079

488. Mojzesová, M.; Mečiarová, M.; Almássy, A.; Marti, R.; Šebesta, R. Chem. Pap. 2015, 69, 737-746. doi:10.1515/chempap-2015-0020

489. Takeda, K.; Oohara, T.; Shimada, N.; Nambu, H.; Hashimoto, S. Chem. - Eur. J. 2011, 17, 13992-13998. doi:10.1002/chem.201102733

490. Chandrasekhar, D.; Borra, S.; Nanubolu, J. B.; Maurya, R. A. Org. Lett. 2016, 18, 2974-2977. doi:10.1021/acs.orglett.6b01321

491.Rincón, J. A.; Mateos, C.; García-Losada, P.; Mergott, D. J. Org. Process Res. Dev. 2015, 19, 347-351. doi:10.1021/op500350y

492.Sarkar, D.; Bera, N.; Ghosh, S. Eur. J. Org. Chem. 2020, 1310-1326. doi:10.1002/ejoc.201901143

493. Namyslo, J. C.; Kaufmann, D. E. Chem. Rev. 2003, 103, 1485-1538. doi:10.1021/cr010010y

494.Crimmins, M. T.; Reinhold, T. L. Enone Olefin [2 + 2] Photochemical Cycloadditions. Organic Reactions; John Wiley \& Sons: Hoboken, NJ, USA, 1993; pp 297-588. doi:10.1002/0471264180.or044.02

495.Levterov, V. V.; Michurin, O.; Borysko, P. O.; Zozulya, S.; Sadkova, I. V.; Tolmachev, A. A.; Mykhailiuk, P. K. J. Org. Chem. 2018, 83, 14350-14361. doi:10.1021/acs.joc.8b02071

496.Knowles, J. P.; Elliott, L. D.; Booker-Milburn, K. I. Beilstein J. Org. Chem. 2012, 8, 2025-2052. doi:10.3762/bjoc.8.229

497.Sambiagio, C.; Noël, T. Trends Chem. 2020, 2, 92-106. doi:10.1016/j.trechm.2019.09.003

498. Mukae, H.; Maeda, H.; Nashihara, S.; Mizuno, K. Bull. Chem. Soc. Jpn. 2007, 80, 1157-1161. doi:10.1246/bcsj.80.1157

499. Vasudevan, A.; Villamil, C.; Trumbull, J.; Olson, J.; Sutherland, D.; Pan, J.; Djuric, S. Tetrahedron Lett. 2010, 51, 4007-4009. doi:10.1016/j.tetlet.2010.05.119

500.Bachollet, S.; Terao, K.; Aida, S.; Nishiyama, Y.; Kakiuchi, K.; Oelgemöller, M. Beilstein J. Org. Chem. 2013, 9, 2015-2021. doi:10.3762/bjoc.9.237

501.Poscharny, K.; Fabry, D. C.; Heddrich, S.; Sugiono, E.; Liauw, M. A.; Rueping, M. Tetrahedron 2018, 74, 3171-3175. doi:10.1016/j.tet.2018.04.019
502. Fukuyama, T.; Hino, Y.; Kamata, N.; Ryu, I. Chem. Lett. 2004, 33, 1430-1431. doi:10.1246/cl.2004.1430

503. Telmesani, R.; Park, S. H.; Lynch-Colameta, T.; Beeler, A. B. Angew. Chem., Int. Ed. 2015, 54, 11521-11525. doi:10.1002/anie.201504454

504. Telmesani, R.; White, J. A. H.; Beeler, A. B. ChemPhotoChem 2018, 2, 865-869. doi:10.1002/cptc.201800081

505.Schachtner, J.; Bayer, P.; Jacobi von Wangelin, A. Beilstein J. Org. Chem. 2016, 12, 1798-1811. doi:10.3762/bjoc.12.170

506. Ratković, A.; Marinić, Ž.; Škorić, I. J. Mol. Struct. 2018, 1168, 165-174. doi:10.1016/j.molstruc.2018.05.027

507.Elliott, L. D.; Knowles, J. P.; Koovits, P. J.; Maskill, K. G.; Ralph, M. J.; Lejeune, G.; Edwards, L. J.; Robinson, R. I.; Clemens, I. R.; Cox, B.; Pascoe, D. D.; Koch, G.; Eberle, M.; Berry, M. B.; Booker-Milburn, K. I. Chem. - Eur. J. 2014, 20, 15226-15232. doi:10.1002/chem.201404347

508. Ralph, M.; Ng, S.; Booker-Milburn, K. I. Org. Lett. 2016, 18, 968-971. doi:10.1021/acs.orglett.6b00067

509. DeLaney, E. N.; Lee, D. S.; Elliott, L. D.; Jin, J.; Booker-Milburn, K. I.; Poliakoff, M.; George, M. W. Green Chem. 2017, 19, 1431-1438. doi:10.1039/c6gc02888h

510.Maskill, K. G.; Knowles, J. P.; Elliott, L. D.; Alder, R. W.; Booker-Milburn, K. I. Angew. Chem., Int. Ed. 2013, 52, 1499-1502. doi:10.1002/anie.201208892

511. Blackham, E. E.; Booker-Milburn, K. I. Angew. Chem., Int. Ed. 2017, 56, 6613-6616. doi:10.1002/anie.201701775

512. Yu, W. L.; Nunns, T.; Richardson, J.; Booker-Milburn, K. I. Org. Lett. 2018, 20, 1272-1274. doi:10.1021/acs.orglett.7b03960

513. Nakano, M.; Nishiyama, Y.; Tanimoto, H.; Morimoto, T.; Kakiuchi, K. Org. Process Res. Dev. 2016, 20, 1626-1632. doi:10.1021/acs.oprd.6b00181

514.Sender, M.; Ziegenbalg, D. Chem. Ing. Tech. 2017, 89, 1159-1173. doi:10.1002/cite.201600191

515. Williams, J. D.; Nakano, M.; Gérardy, R.; Rincón, J. A.; de Frutos, Ó.; Mateos, C.; Monbaliu, J.-C. M.; Kappe, C. O. Org. Process Res. Dev. 2019, 23, 78-87. doi:10.1021/acs.oprd.8b00375

516. Yang, C.; Li, R.; Zhang, K. A. I.; Lin, W.; Landfester, K.; Wang, X. Nat. Commun. 2020, 11, 1239. doi:10.1038/s41467-020-14983-w

517. Elliott, L. D.; Berry, M.; Harji, B.; Klauber, D.; Leonard, J.; Booker-Milburn, K. I. Org. Process Res. Dev. 2016, 20, 1806-1811. doi:10.1021/acs.oprd.6b00277

518. Kurahashi, K.; Takemoto, Y.; Takasu, K. ChemSusChem 2012, 5, 270-273. doi:10.1002/cssc.201100373

519. Hafez, A. M.; Taggi, A. E.; Wack, H.; Drury, W. J.; Lectka, T. Org. Lett. 2000, 2, 3963-3965. doi:10.1021/ol006659r

520. Hafez, A. M.; Taggi, A. E.; Lectka, T. Chem. - Eur. J. 2002, 8, 4114-4119. doi:10.1002/1521-3765(20020916)8:18<4114::aid-chem4114>3.0.co; 2-q

521. Hafner, A.; Ley, S. V. Synlett 2015, 26, 1470-1474. doi:10.1055/s-0034-1380679

522. Battilocchio, C.; lannucci, G.; Wang, S.; Godineau, E.; Kolleth, A.; De Mesmaeker, A.; Ley, S. V. React. Chem. Eng. 2017, 2, 295-298. doi:10.1039/c7re00020k

523. Ueda, M.; Hayama, M.; Hashishita, H.; Munechika, A.; Fukuyama, T. Eur. J. Org. Chem. 2019, 6764-6769. doi:10.1002/ejoc.201901072

524. García-Lacuna, J.; Domínguez, G.; Blanco-Urgoiti, J.; Pérez-Castells, J. Org. Lett. 2018, 20, 5219-5223. doi:10.1021/acs.orglett.8b02168 
525. Saaby, S.; Baxendale, I. R.; Ley, S. V. Org. Biomol. Chem. 2005, 3, 3365. doi:10.1039/b509540a

526. Wilson, N. S.; Sarko, C. R.; Roth, G. P. Org. Process Res. Dev. 2004, 8, 535-538. doi:10.1021/op034181b

527.Scriabine, I. Alkylaromatic Aldehydes and Their Preparation. U.S. Pat. Appl. US3023247A, Feb 27, 1962.

528. Wood, T.; Heilweil, E. Process for Producing 1,1,3,4,4,6-Hexamethyl-1,2,3,4-Tetrahydronapthalene (Hmt). U.S. Pat. Appl. US3856875A, Feb 27, 1962.

529. Polak, E. H. A Process for the Preparation of Musky Odor Polyalkylindanderivaten. Ger. Patent DE1045399B, Dec 4, 1958.

530. Calloway, N. O. Chem. Rev. 1935, 17, 327-392. doi:10.1021/cr60058a002

531. Rueping, M.; Nachtsheim, B. J. Beilstein J. Org. Chem. 2010, 6 , No. 6. doi:10.3762/bjoc.6.6

532. Arata, K.; Toyoshima, I. J. Catal. 1977, 47, 109-112. doi:10.1016/0021-9517(77)90156-7

533. Hitzler, M. G.; Smail, F. R.; Poliakoff, M.; Hitzler, M. G.; Ross, S. K. Chem. Commun. 1998, 359-360. doi:10.1039/a707149c

534.Bogdan, A. R.; Poe, S. L.; Kubis, D. C.; Broadwater, S. J.; McQuade, D. T. Angew. Chem., Int. Ed. 2009, 48, 8547-8550. doi:10.1002/anie.200903055

535.Snead, D. R.; Jamison, T. F. Angew. Chem., Int. Ed. 2015, 54, 983-987. doi:10.1002/anie.201409093

536. Tietze, L. F.; Liu, D. ARKIVOC 2008, No. viii, 193-210. doi:10.3998/ark.5550190.0009.816

537.Li, W.; Yang, S.; Guo, X.; He, G.; Jin, H. Chin. J. Chem. Eng. 2018, 26, 1307-1311. doi:10.1016/j.cjche.2018.01.004

538. Fang, H.; Xiao, Q.; Wu, F.; Floreancig, P. E.; Weber, S. G. J. Org. Chem. 2010, 75, 5619-5626. doi:10.1021/jo100981e

539. Hone, C. A.; Boyd, A.; O'Kearney-McMullan, A.; Bourne, R. A.; Muller, F. L. React. Chem. Eng. 2019, 4, 1565-1570. doi:10.1039/c9re00180h

540.Koo, H.; Kim, H. Y.; Oh, K. Org. Chem. Front. 2019, 6, 1868-1872. doi:10.1039/c9qo00217k

541.Koo, H.; Kim, H. Y.; Oh, K. Org. Lett. 2019, 21, 10063-10068. doi:10.1021/acs.orglett.9b04010

542.Wang, X.; Wang, S.; Ge, X.; Qui, T.; Wang, H.; Wang, Q.; Chen, J.; Huang, Z.; Ye, C.; Li, L. Micro-Continuous Flow Process for Producing Musk Tonalide. Chin. Pat. Appl. CN110950746A, 2019.

543. Aribert, N.; Camy, S.; Peres Lucchese, Y.; Condoret, J.-S.; Cognet, P. Int. J. Chem. React. Eng. 2010, 8. doi:10.2202/1542-6580.2183

544.Chen, Z.; Chen, W.; Tong, T.; Zeng, A. J. Mol. Catal. A: Chem. 2015, 396, 231-238. doi:10.1016/j.molcata.2014.09.038

545.Rao, X.; Ishitani, H.; Yoo, W.-J.; Kobayashi, S. Asian J. Org. Chem. 2019, 8, 316-319. doi:10.1002/ajoc.201900012

546.Fang, Z.; He, W.; Tu, T.; Lv, N.; Qiu, C.; Li, X.; Zhu, N.; Wan, L.; Guo, K. Chem. Eng. J. 2018, 331, 443-449. doi:10.1016/j.cej.2017.09.001

547.Ji, P.; Feng, X.; Oliveres, P.; Li, Z.; Murakami, A.; Wang, C.; Lin, W. J. Am. Chem. Soc. 2019, 141, 14878-14888. doi:10.1021/jacs.9b07891

548.Kang, Q.; Zhao, Z.-A.; You, S.-L. J. Am. Chem. Soc. 2007, 129, 1484-1485. doi:10.1021/ja067417a

549. Osorio-Planes, L.; Rodríguez-Escrich, C.; Pericàs, M. A. Chem. - Eur. J. 2014, 20, 2367-2372. doi:10.1002/chem.201303860

550.Rodríguez-Rodríguez, M.; Maestro, A.; Andrés, J. M.; Pedrosa, R. Adv. Synth. Catal. 2020, 362, 2744-2754. doi:10.1002/adsc.202000238
551.Bahrmann, H.; Cornils, B.; Diekhaus, G.; Kascha, W.; Weber, J. Verfahren Zur Herstellung von Aldehyden. Ger. Pat. Appl. DE2952278A1, Oct 16, 1980.

552. Frisch, A. C.; Webb, P. B.; Zhao, G.; Muldoon, M. J.; Pogorzelec, P. J.; Cole-Hamilton, D. J. Dalton Trans. 2007, 5531. doi:10.1039/b712683b

553. Webb, P. B.; Sellin, M. F.; Kunene, T. E.; Williamson, S.; Slawin, A. M. Z.; Cole-Hamilton, D. J. J. Am. Chem. Soc. 2003, 125, 15577-15588. doi:10.1021/ja035967s

554.Burguete, M. I.; García-Verdugo, E.; Luis, S. V. Beilstein J. Org. Chem. 2011, 7, 1347-1359. doi:10.3762/bjoc.7.159

555. Wolf, P.; Logemann, M.; Schörner, M.; Keller, L.; Haumann, M.; Wessling, M. RSC Adv. 2019, 9, 27732-27742. doi:10.1039/c9ra04830h

556. Kasinathan, S.; Bourne, S. L.; Tolstoy, P.; Koos, P.; O’Brien, M.; Bates, R. W.; Baxendale, I. R.; Ley, S. V. Synlett 2011, 2648-2651. doi:10.1055/s-0031-1289292

557. Masui, H.; Honda, E.; Niitsu, S.; Shoji, M.; Takahashi, T. Int. J. Org. Chem. 2018, 08, 135-141. doi:10.4236/ijoc.2018.81009

558. Keybl, J.; Jensen, K. F. Ind. Eng. Chem. Res. 2011, 50, 11013-11022. doi:10.1021/ie200936b

559.Zhu, C.; Raghuvanshi, K.; Coley, C. W.; Mason, D.; Rodgers, J.; Janka, M. E.; Abolhasani, M. Chem. Commun. 2018, 54, 8567-8570. doi:10.1039/c8cc04650f

560. Raghuvanshi, K.; Zhu, C.; Ramezani, M.; Menegatti, S.; Santiso, E. E.; Mason, D.; Rodgers, J.; Janka, M. E.; Abolhasani, M. ACS Catal. 2020, 10, 7535-7542. doi:10.1021/acscatal.0c01515

561. Kaiser, N. M.; Jokiel, M.; McBride, K.; Flassig, R. J.; Sundmacher, K. Ind. Eng. Chem. Res. 2017, 56, 11507-11518. doi:10.1021/acs.iecr.7b01939

562.Jokiel, M.; Kaiser, N. M.; Kováts, P.; Mansour, M.; Zähringer, K.; Nigam, K. D. P.; Sundmacher, K. Chem. Eng. J. 2019, 377, 120060. doi:10.1016/j.cej.2018.09.221

563.Wang, S.; Zhang, J.; Peng, F.; Tang, Z.; Sun, Y. Ind. Eng. Chem. Res. 2020, 59, 88-98. doi:10.1021/acs.iecr.9b05350

564. Watkins, A. L.; Landis, C. R. J. Am. Chem. Soc. 2010, 132, 10306-10317. doi:10.1021/ja909619a

565. Cunillera, A.; Blanco, C.; Gual, A.; Marinkovic, J. M.; Garcia-Suarez, E. J.; Riisager, A.; Claver, C.; Ruiz, A.; Godard, C. ChemCatChem 2019, 11, 2195-2205. doi:10.1002/cctc.201900211

566. Gutmann, R.; Gscheidmeier, M.; Wiesmüller, J.; Riedel, A. Process for the Contiunuous Preparation of Terpene Esters. U.S. Patent US5596127, Jan 21, 1997.

567.Zheng, H.; Yan, Z.; Chu, S.; Chen, J. Chem. Eng. Process. 2018, 134, 1-8. doi:10.1016/j.cep.2018.10.005

568. Adarme, C. A. A.; Leão, R. A. C.; de Souza, S. P.; Itabaiana, I., Jr.; de Souza, R. O. M. A.; Rezende, C. M. Mol. Catal. 2018, 453, 39-46. doi:10.1016/j.mcat.2018.04.007

569. Okuno, Y.; Isomura, S.; Kamakura, T.; Sano, F.; Tamahori, K.; Goto, T.; Hayashida, T.; Kitagawa, Y.; Fukuhara, A.; Takeda, K. ChemSusChem 2015, 8, 1711-1715. doi:10.1002/cssc.201500092

570. He, P.; Haswell, S. J.; Fletcher, P. D. I.; Kelly, S. M.; Mansfield, A. J. Flow Chem. 2012, 2, 47-51. doi:10.1556/jfc-d-12-00002

571.Baumann, M.; Baxendale, I. R.; Filipponi, P.; Hu, T. Org. Process Res. Dev. 2017, 21, 2052-2059. doi:10.1021/acs.oprd.7b00328

572.Li, X.; Chen, A.; Zhou, Y.; Huang, L.; Fang, Z.; Gan, H.; Guo, K. J. Flow Chem. 2015, 5, 82-86. doi:10.1556/1846.2014.00043 
573.Csajági, C.; Szatzker, G.; Rita Tőke, E.; Ürge, L.; Darvas, F.; Poppe, L. Tetrahedron: Asymmetry 2008, 19, 237-246. doi:10.1016/j.tetasy.2008.01.002

574.Calmanti, R.; Galvan, M.; Amadio, E.; Perosa, A.; Selva, M. ACS Sustainable Chem. Eng. 2018, 6, 3964-3973. doi:10.1021/acssuschemeng.7b04297

575. Contente, M. L.; Farris, S.; Tamborini, L.; Molinari, F.; Paradisi, F. Green Chem. 2019, 21, 3263-3266. doi:10.1039/c9gc01374a

576. Battilocchio, C.; Deadman, B. J.; Nikbin, N.; Kitching, M. O.; Baxendale, I. R.; Ley, S. V. Chem. - Eur. J. 2013, 19, 7917-7930. doi:10.1002/chem.201300696

577. Trnka, T. M.; Grubbs, R. H. Acc. Chem. Res. 2001, 34, 18-29. doi:10.1021/ar000114f

578. Mol, J. C. J. Mol. Catal. A: Chem. 2004, 213, 39-45. doi:10.1016/j.molcata.2003.10.049

579.Sinclair, F.; Alkattan, M.; Prunet, J.; Shaver, M. P. Polym. Chem. 2017, 8, 3385-3398. doi:10.1039/c7py00340d

580. Hughes, D.; Wheeler, P.; Ene, D. Org. Process Res. Dev. 2017, 21 , 1938-1962. doi:10.1021/acs.oprd.7b00319

581. Yu, M.; Lou, S.; Gonzalez-Bobes, F. Org. Process Res. Dev. 2018, 22, 918-946. doi:10.1021/acs.oprd.8b00093

582. Grela, K.; Harutyunyan, S.; Michrowska, A. Angew. Chem., Int. Ed. 2002, 41, 4038-4040. doi:10.1002/1521-3773(20021104)41:21<4038::aid-anie4038>3.0.co; 2-0

583. Kadyrov, R. Chem. - Eur. J. 2013, 19, 1002-1012. doi:10.1002/chem.201202207

584.Gawin, R.; Tracz, A.; Chwalba, M.; Kozakiewicz, A.; Trzaskowski, B.; Skowerski, K. ACS Catal. 2017, 7, 5443-5449. doi:10.1021/acscatal.7b00597

585.Dumas, A.; Colombel-Rouen, S.; Curbet, I.; Forcher, G.; Tripoteau, F.; Caijo, F.; Queval, P.; Rouen, M.; Baslé, O.; Mauduit, M. Catal. Sci. Technol. 2019, 9, 436-443. doi:10.1039/c8cy02115e

586. Dörrich, S.; Ulmer, A.; Mahler, C.; Burschka, C.; Baus, J. A.; Tacke, R.; Chai, A.; Ding, C.; Zou, Y.; Brunner, G.; Goeke, A.; Kraft, P. Eur. J. Inorg. Chem. 2014, 4394-4407. doi:10.1002/ejic.201402597

587. Lovchik, M. A.; Kraft, P. Chem. - Eur. J. 2017, 23, 4590-4596. doi:10.1002/chem.201605378

588. Williams, A. S. Synthesis 1999, 1707-1723. doi:10.1055/s-1999-3581

589. Wiegers, W. J.; Van Loveren, A. G.; Hanna, M. R.; Luccarelli, D., Jr.; Bowen, D. R.; Vock, M. H. Macrocyclic Carbonates, Processes for Preparing Same, Organoleptic Uses Thereof and Intermediates Used in Said Process. U.S. Pat. Appl. US4490544A, Dec 25, 1984.

590. Tsuji, J.; Hashiguchi, S. Tetrahedron Lett. 1980, 21, 2955-2958. doi:10.1016/0040-4039(80)88007-5

591. Kraft, P.; Berthold, C. Synthesis 2008, 543-550. doi:10.1055/s-2008-1032135

592. Lehr, K.; Fürstner, A. Tetrahedron 2012, 68, 7695-7700. doi:10.1016/j.tet.2012.05.085

593.Narula, A. P. S.; Arruda, E. M.; Amorelli, B.; Schiet, F. T. 3-Methyl-6-Cyclohexadecen-1-One and Its Use in Perfume Compositions. U.S. Patent US8450265B2, May 28, 2013.

594.Shen, J.; Shi, Y.; Tian, W. Chin. J. Chem. 2015, 33, 683-687. doi:10.1002/cjoc.201400862

595.Callejo, R.; Corr, M. J.; Yang, M.; Wang, M.; Cordes, D. B.; Slawin, A. M. Z.; O'Hagan, D. Chem. - Eur. J. 2016, 22, 8137-8151. doi:10.1002/chem.201600519

596. Sytniczuk, A.; Leszczyńska, A.; Kajetanowicz, A.; Grela, K. ChemSusChem 2018, 11, 3157-3166. doi:10.1002/cssc.201801463
597. Reckziegel, A.; Wohrle, I.; Sonnenberg, S. (Z)-7-Cyclohexadecen-1-One as an Odorant. U.S. Pat. Appl. US0137120A1, May 28, 2013.

598. Michrowska, A.; Wawrzyniak, P.; Grela, K. Eur. J. Org. Chem. 2004, 2053-2056. doi:10.1002/ejoc.200300768

599. Mathys, M.; Kraft, P. Chem. Biodiversity 2014, 11, 1597-1607. doi:10.1002/cbdv.201400011

600.Comer, E.; Organ, M. G. J. Am. Chem. Soc. 2005, 127, 8160-8167. doi:10.1021/ja0512069

601. Ahmed-Omer, B.; Barrow, D. A.; Wirth, T. ARKIVOC 2011, No. iv, 26-36. doi:10.3998/ark.5550190.0012.404

602.Drop, M.; Bantreil, X.; Grychowska, K.; Mahoro, G. U.; Colacino, E.; Pawłowski, M.; Martinez, J.; Subra, G.; Zajdel, P.; Lamaty, F. Green Chem. 2017, 19, 1647-1652. doi:10.1039/c7gc00235a

603. Alexander, K. A.; Paulhus, E. A.; Lazarus, G. M. L.; Leadbeater, N. E. J. Organomet. Chem. 2016, 812, 74-80. doi:10.1016/j.jorganchem.2015.09.018

604.Morin, É.; Sosoe, J.; Raymond, M.; Amorelli, B.; Boden, R. M.; Collins, S. K. Org. Process Res. Dev. 2019, 23, 283-287. doi:10.1021/acs.oprd.8b00450

605. Michrowska, A.; Mennecke, K.; Kunz, U.; Kirschning, A.; Grela, K. J. Am. Chem. Soc. 2006, 128, 13261-13267. doi:10.1021/ja063561k

606. Cabrera, J.; Padilla, R.; Bru, M.; Lindner, R.; Kageyama, T.; Wilckens, K.; Balof, S. L.; Schanz, H.-J.; Dehn, R.; Teles, J. H.; Deuerlein, S.; Müller, K.; Rominger, F.; Limbach, M. Chem. - Eur. J. 2012, 18, 14717-14724. doi:10.1002/chem.201202248

607.Bru, M.; Dehn, R.; Teles, J. H.; Deuerlein, S.; Danz, M.; Müller, I. B.; Limbach, M. Chem. - Eur. J. 2013, 19, 11661-11671. doi:10.1002/chem.201203893

608. Solodenko, W.; Doppiu, A.; Frankfurter, R.; Vogt, C.; Kirschning, A. Aust. J. Chem. 2013, 66, 183-191. doi:10.1071/ch12434

609. Balcar, H.; Žilková, N.; Kubů, M.; Polášek, M.; Zedník, J. Catal. Today 2018, 304, 127-134. doi:10.1016/j.cattod.2017.09.049

610.Lim, J.; Seong Lee, S.; Ying, J. Y. Chem. Commun. 2010, 46, 806-808. doi:10.1039/b917986k

611.Riva, E.; Rencurosi, A.; Gagliardi, S.; Passarella, D.; Martinelli, M. Chem. - Eur. J. 2011, 17, 6221-6226. doi:10.1002/chem.201100300

612.Gmeiner, J.; Seibicke, M.; Lang, C.; Gärtner, U.; Trapp, O. Adv. Synth. Catal. 2014, 356, 2081-2087. doi:10.1002/adsc.201301182

613.Borré, E.; Rouen, M.; Laurent, I.; Magrez, M.; Caijo, F.; Crévisy, C.; Solodenko, W.; Toupet, L.; Frankfurter, R.; Vogt, C.; Kirschning, A.; Mauduit, M. Chem. - Eur. J. 2012, 18, 16369-16382. doi:10.1002/chem.201201589

614.Selva, M.; Guidi, S.; Perosa, A.; Signoretto, M.; Licence, P.; Maschmeyer, T. Green Chem. 2012, 14, 2727-2737. doi:10.1039/c2gc35983a

615.Duque, R.; Öchsner, E.; Clavier, H.; Caijo, F.; Nolan, S. P.; Mauduit, M.; Cole-Hamilton, D. J. Green Chem. 2011, 13, 1187-1195. doi:10.1039/c1gc15048k

616. Schoeps, D.; Buhr, K.; Dijkstra, M.; Ebert, K.; Plenio, H. Chem. - Eur. J. 2009, 15, 2960-2965. doi:10.1002/chem.200802153

617.Rabiller-Baudry, M.; Nasser, G.; Renouard, T.; Delaunay, D.; Camus, M. Sep. Purif. Technol. 2013, 116, 46-60. doi:10.1016/j.seppur.2013.04.052

618.Ormerod, D.; Bongers, B.; Porto-Carrero, W.; Giegas, S.; Vijt, G.; Lefevre, N.; Lauwers, D.; Brusten, W.; Buekenhoudt, A. RSC Adv. 2013, 3, 21501-21510. doi:10.1039/c3ra44860f

619. O'Neal, E. J.; Jensen, K. F. ChemCatChem 2014, 6, 3004-3011. doi:10.1002/cctc.201402368 
620.Monfette, S.; Eyholzer, M.; Roberge, D. M.; Fogg, D. E. Chem. - Eur. J. 2010, 16, 11720-11725.

doi:10.1002/chem.201001210

621.Skowerski, K.; Czarnocki, S. J.; Knapkiewicz, P. ChemSusChem 2014, 7, 536-542. doi:10.1002/cssc.201300829

622.Ley, S. V.; Ingham, R. J.; O’Brien, M.; Browne, D. L. Beilstein J. Org. Chem. 2013, 9, 1051-1072. doi:10.3762/bjoc.9.118

623.Kreutz, J. E.; Shukhaev, A.; Du, W.; Druskin, S.; Daugulis, O.; Ismagilov, R. F. J. Am. Chem. Soc. 2010, 132, 3128-3132. doi:10.1021/ja909853x

624. Rogers, L.; Jensen, K. F. Green Chem. 2019, 21, 3481-3498. doi:10.1039/c9gc00773c

625. Hughes, D. L. Org. Process Res. Dev. 2018, 22, 13-20. doi:10.1021/acs.oprd.7b00363

626. Testa, C. J.; Hu, C.; Shvedova, K.; Wu, W.; Sayin, R.; Casati, F.; Halkude, B. S.; Hermant, P.; Shen, D. E.; Ramnath, A.; Su, Q.; Born, S. C.; Takizawa, B.; Chattopadhyay, S.; O'Connor, T. F.; Yang, X.; Ramanujam, S.; Mascia, S. Org. Process Res. Dev. 2020, 24, 2874-2889. doi:10.1021/acs.oprd.0c00383

\section{License and Terms}

This is an Open Access article under the terms of the Creative Commons Attribution License (https://creativecommons.org/licenses/by/4.0). Please note that the reuse, redistribution and reproduction in particular requires that the author(s) and source are credited and that individual graphics may be subject to special legal provisions.

The license is subject to the Beilstein Journal of Organic Chemistry terms and conditions:

(https://www.beilstein-journals.org/bjoc/terms)

The definitive version of this article is the electronic one which can be found at: https://doi.org/10.3762/bjoc. 17.90 